\begin{tabular}{c} 
OAK RIDGE \\
NATIONAL LABORATORY \\
\hline
\end{tabular}

MANAGED BY UT-BATTELLE

FOR THE DEPARTMENT OF ENERGY

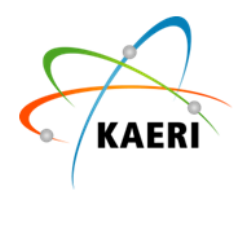

\title{
Fully Ceramic Microencapsulated (FCM) Replacement Fuel for LWRs
}

May 2013

Prepared by Jeffrey J. Powers

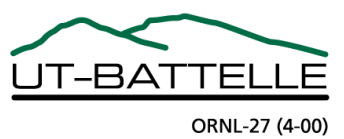




\title{
DOCUMENT AVAILABILITY
}

Reports produced after January 1, 1996, are generally available free via the U.S. Department of Energy (DOE) Information Bridge.

Web site http://www.osti.gov/bridge

Reports produced before January 1, 1996, may be purchased by members of the public from the following source.

\author{
National Technical Information Service \\ 5285 Port Royal Road \\ Springfield, VA 22161 \\ Telephone 703-605-6000 (1-800-553-6847) \\ TDD 703-487-4639 \\ Fax 703-605-6900 \\ E-mail info@ntis.gov \\ Web site http://www.ntis.gov/support/ordernowabout.htm
}

Reports are available to DOE employees, DOE contractors, Energy Technology Data Exchange (ETDE) representatives, and International Nuclear Information System (INIS) representatives from the following source.

Office of Scientific and Technical Information

P.O. Box 62

Oak Ridge, TN 37831

Telephone 865-576-8401

Fax 865-576-5728

E-mail reports@osti.gov

Web site http://www.osti.gov/contact.html

This report was prepared as an account of work sponsored by an agency of the United States Government. Neither the United States Government nor any agency thereof, nor any of their employees, makes any warranty, express or implied, or assumes any legal liability or responsibility for the accuracy, completeness, or usefulness of any information, apparatus, product, or process disclosed, or represents that its use would not infringe privately owned rights. Reference herein to any specific commercial product, process, or service by trade name, trademark, manufacturer, or otherwise, does not necessarily constitute or imply its endorsement, recommendation, or favoring by the United States Government or any agency thereof. The views and opinions of authors expressed herein do not necessarily state or reflect those of the United States Government or any agency thereof. 
ORNL/TM-2013/173

KAERI/TR-5136/2013

Reactor and Nuclear Systems Division

\title{
FULLY CERAMIC MICROENCAPSULATED (FCM) REPLACEMENT FUEL FOR LWRs
}

\author{
J. J. Powers \\ W. J. Lee \\ F. Venneri \\ L. L. Snead \\ C. K. Jo \\ D. H. Hwang \\ J. H. Chun \\ Y. M. Kim \\ K. A. Terrani
}

Date Published: May 2013

\author{
Prepared by \\ OAK RIDGE NATIONAL LABORATORY \\ Oak Ridge, Tennessee 37831-6283 \\ managed by \\ UT-BATTELLE, LLC \\ for the \\ U.S. DEPARTMENT OF ENERGY \\ under contract DE-AC05-00OR22725
}





\section{CONTENTS}

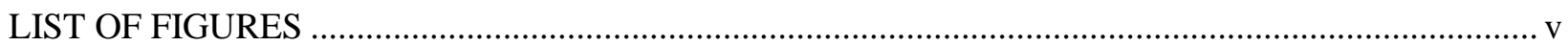

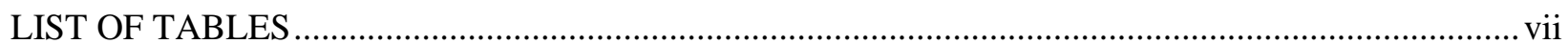

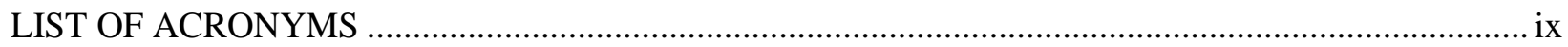

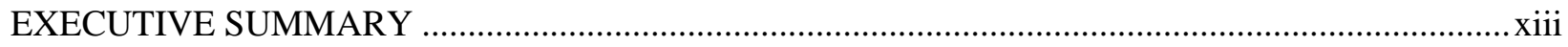

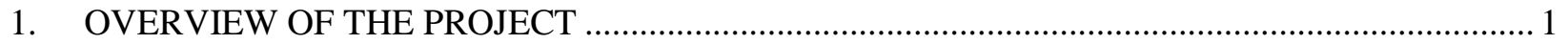

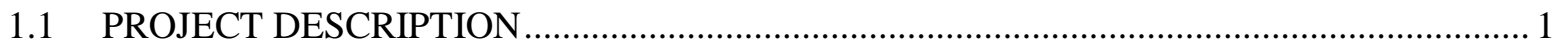

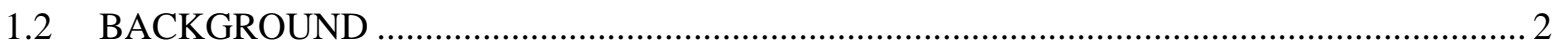

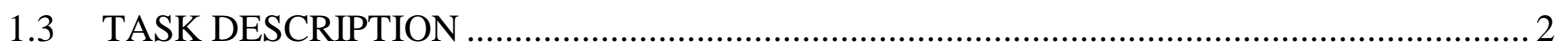

1.3.1 Task 1: Preliminary Neutronics Exploration of FCM Fuel Assemblies and Cores ....... 3

1.3.2 Task 2: Preliminary Core Thermal-Hydraulics Assessment of FCM-Fueled LWR Cores

1.3.3 Task 3: Preliminary Safety Assessment of FCM Fueled LWR Cores ........................... 3

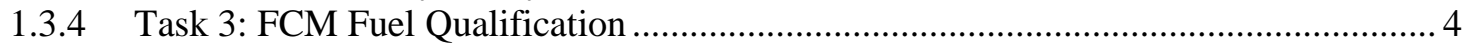

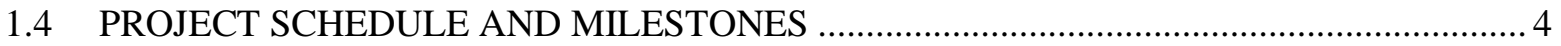

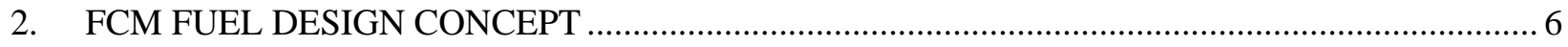

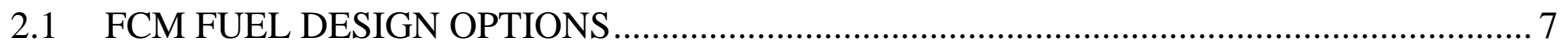

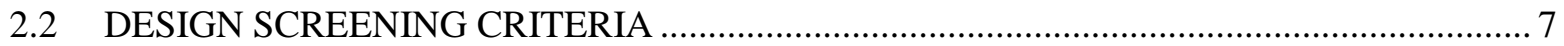

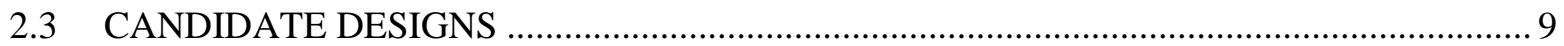

2.3.1 FCM Replacement Fuel for OPR-1000 Core .......................................................... 9

2.3.2 FCM Replacement Fuel for Westinghouse Core ..................................................... 10

3. NEUTRONICS EXPLORATION OF LWR FUEL ASSEMBLIES …......................................... 11

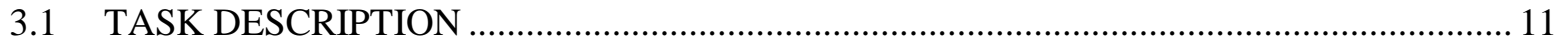

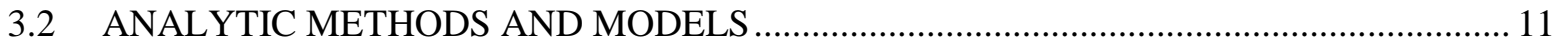

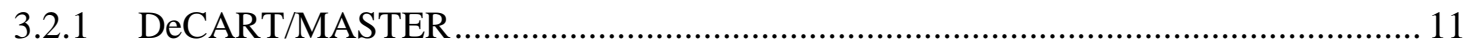

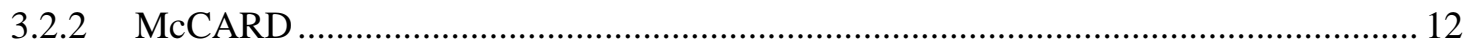

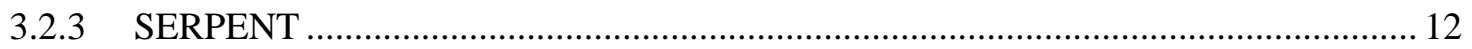

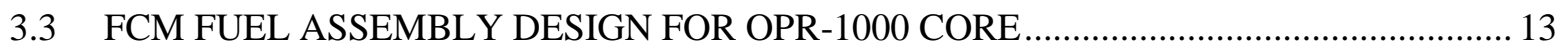

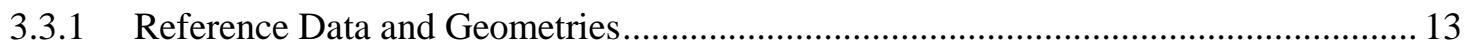

3.3.2 Scoping Analysis for the Fuel Assembly Design Selection...................................... 15

3.3.3 Results of the Fuel Assembly Analysis.................................................................... 19

3.4 FCM FUEL ASSEMBLY DESIGN FOR WESTINGHOUSE CORE .................................. 24

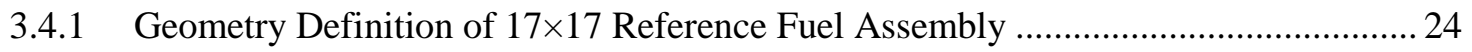

3.4.2 Geometry Definition of $13 \times 13$ FCM Fuel Assembly .............................................. 25

3.4.3 13×13 FCM Fuel Assembly Initial Design Analysis ............................................. 28

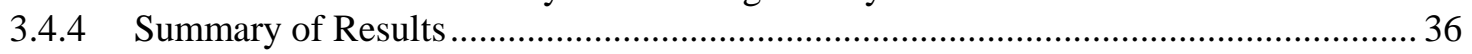

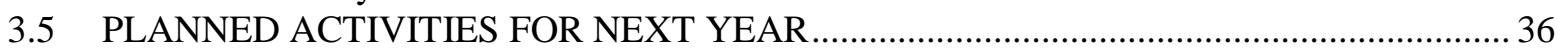

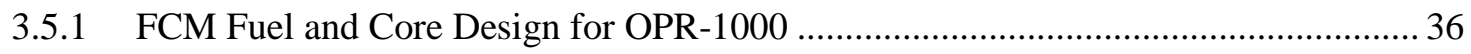

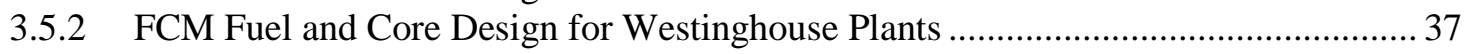

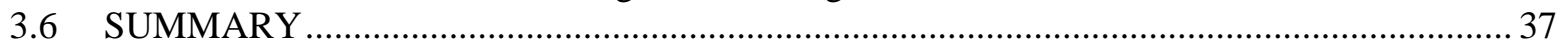

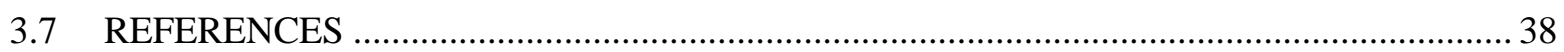

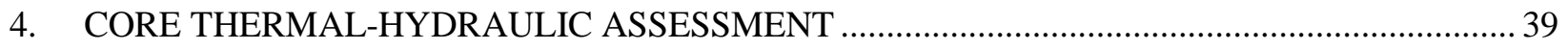

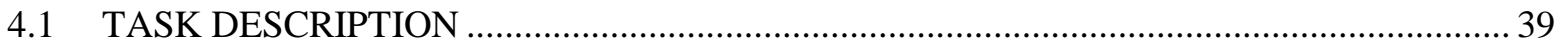

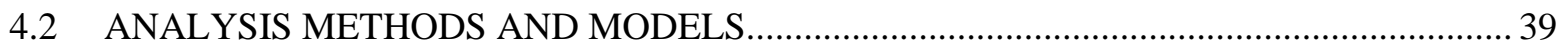

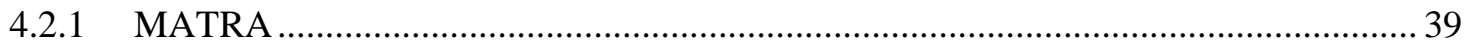

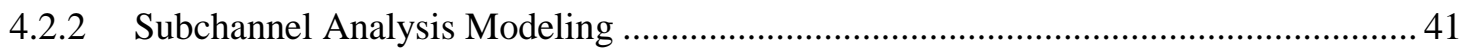

4.3 PRELIMINARY SCOPING ANALYSIS FOR OPR-1000 CORE …................................. 44

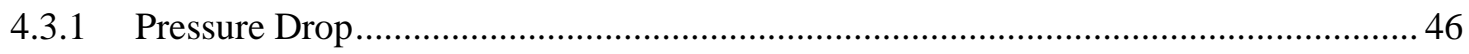

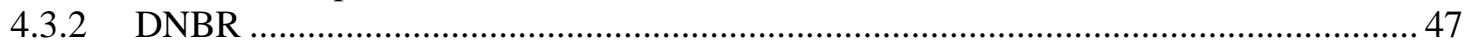




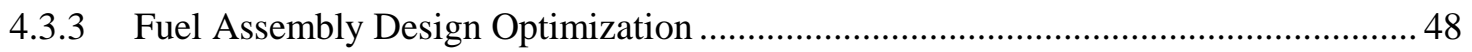

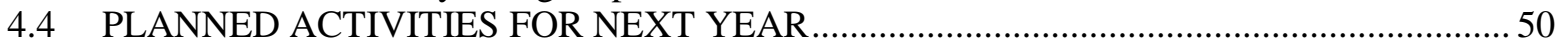

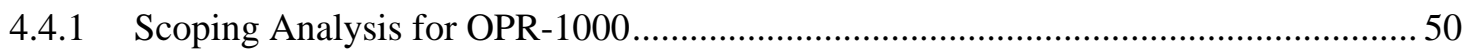

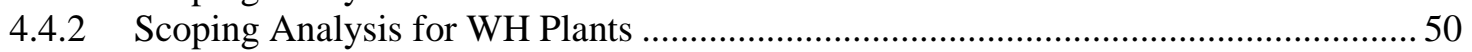

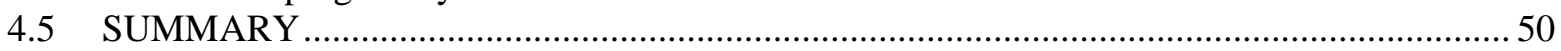

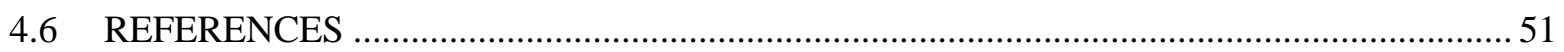

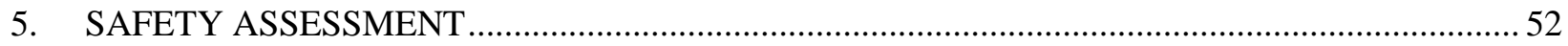

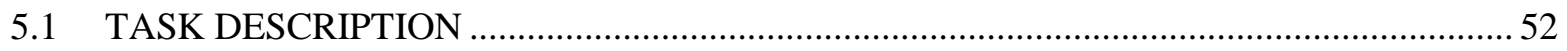

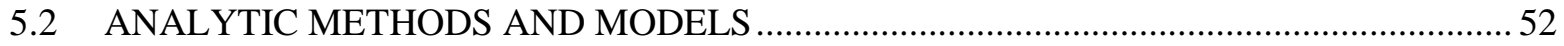

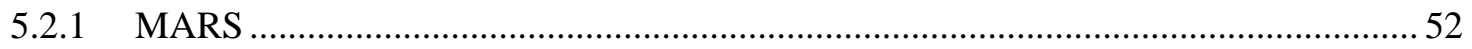

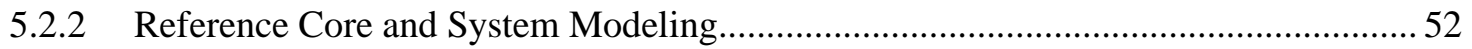

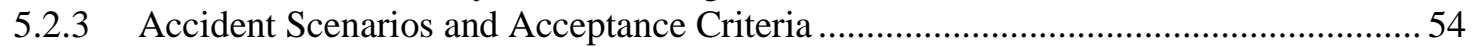

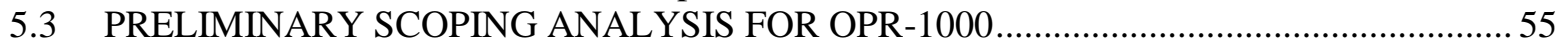

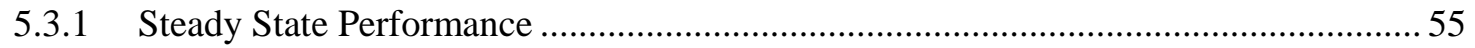

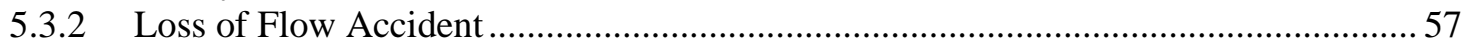

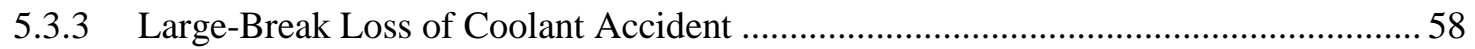

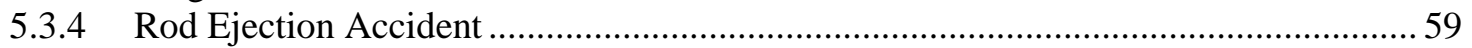

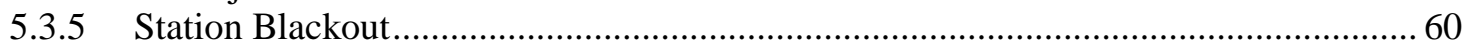

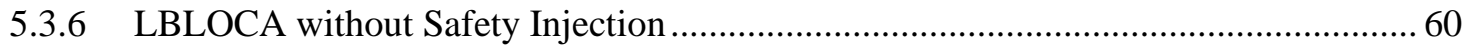

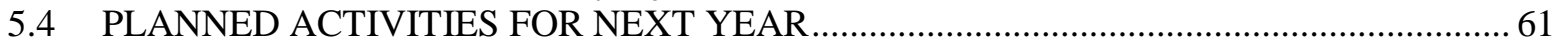

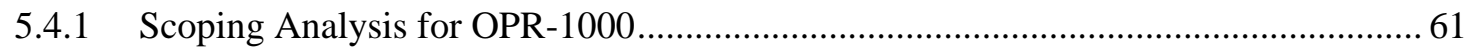

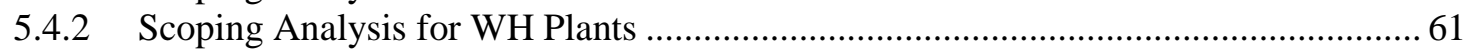

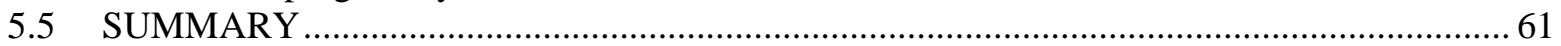

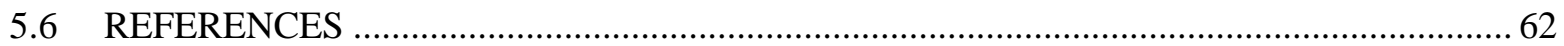

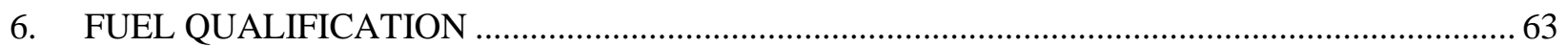

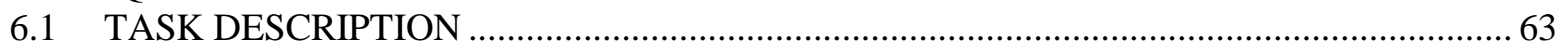

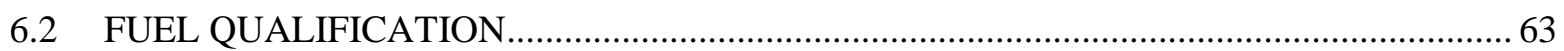

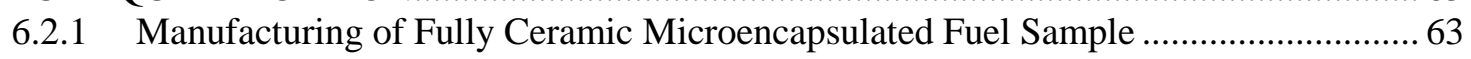

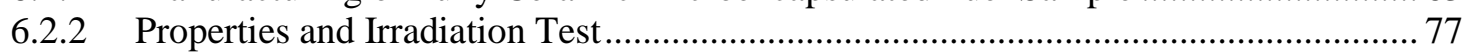

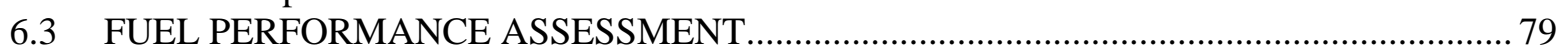

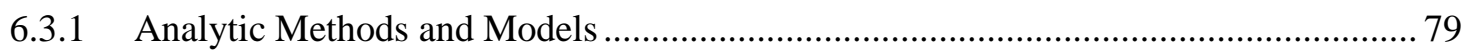

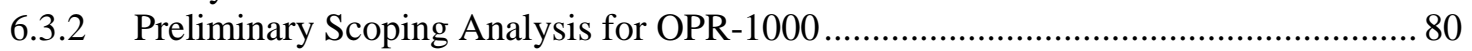

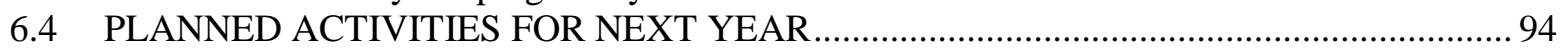

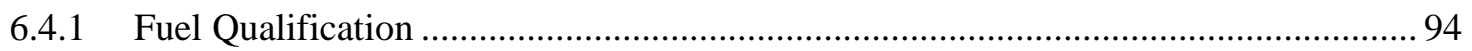

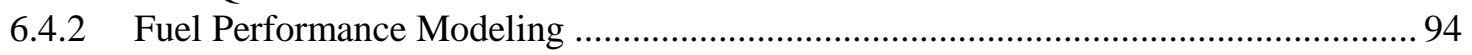

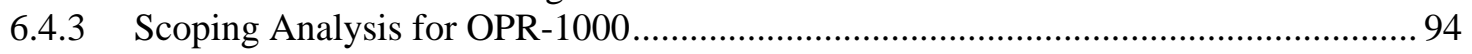

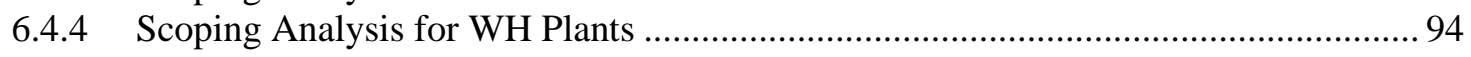

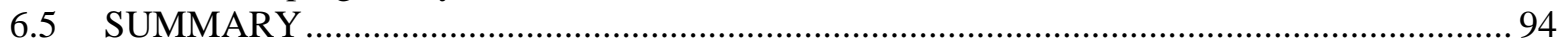

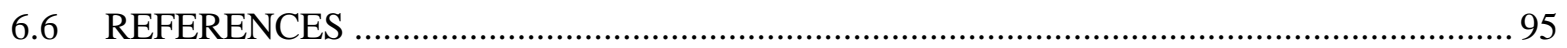

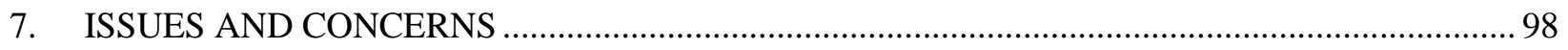

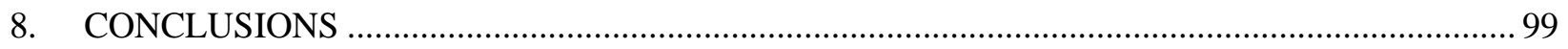




\section{LIST OF FIGURES}

Figure $\quad$ Page

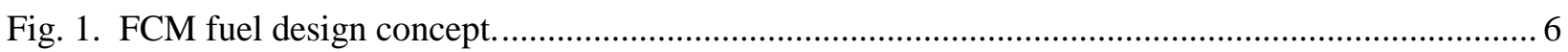

Fig. 2. Candidate $13 \times 13$ FCM UN fuel design for Westinghouse core ................................................ 10

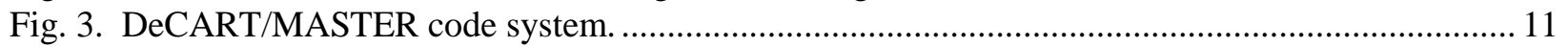

Fig. 4. Configurations of the fuel pin cell and $16 \times 16$ quarter fuel assembly......................................... 14

Fig. 5. Configuration of the FCM fuel pin cell and $12 \times 12$ quarter fuel assembly.................................. 14

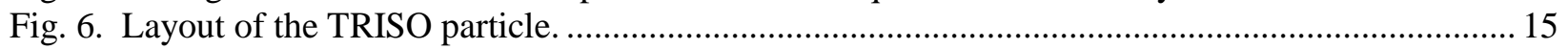

Fig. 7. k-infinity changes with kernel diameter and cladding materials. ............................................. 17

Fig. 8. k-infinity changes with TRISO packing fraction and cladding materials.................................. 18

Fig. 9. Neutron multiplication factors of FCM fuel assembly. .......................................................... 20

Fig. 10. Moderator temperature coefficients (soluble boron concentration $=500 \mathrm{ppm}$ ).................... 21

Fig. 11. Comparison of the neutron spectra for solid $\mathrm{UO}_{2}$ fuel (left) and $\mathrm{UN}$ FCM fuel (right) .............. 21

Fig. 12. Comparison of the MTC with soluble boron concentrations of $0 \mathrm{ppm}$ (left) and $1000 \mathrm{ppm}$

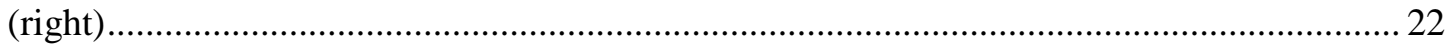

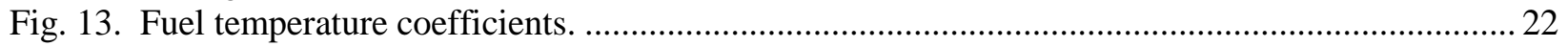

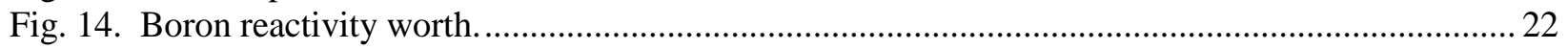

Fig. 15. Effects of burnable poisons in a UN FCM fuel assembly .................................................... 23

Fig. 16. Comparison of the fast neutron fluence of the HTR fuel compact, $\mathrm{LWR}$ solid $\mathrm{UO}_{2}$ fuel,

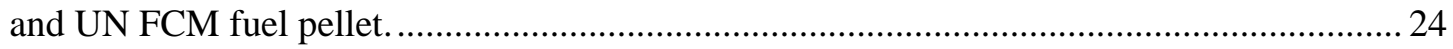

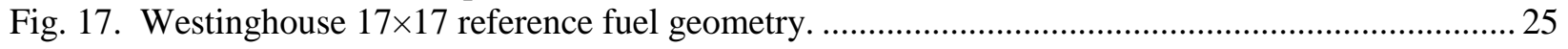

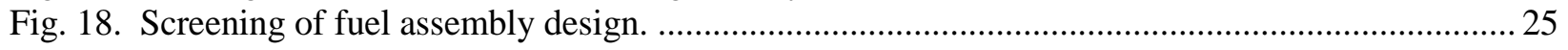

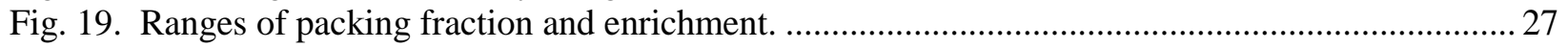

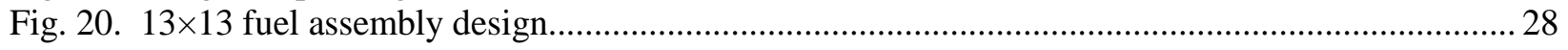

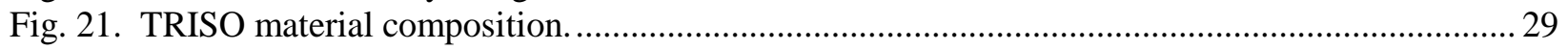

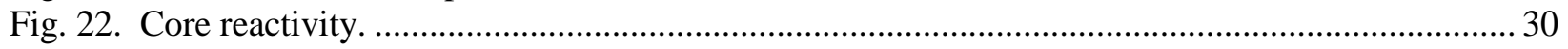

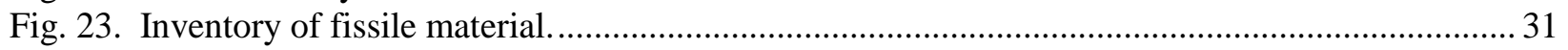

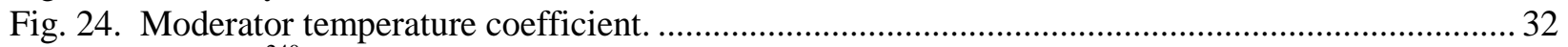

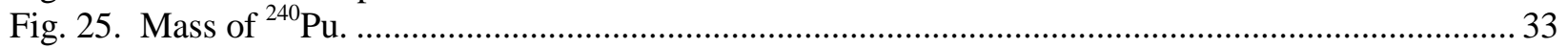

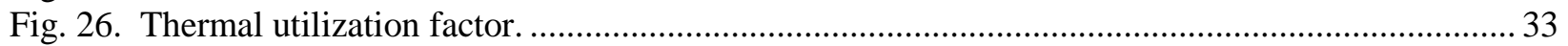

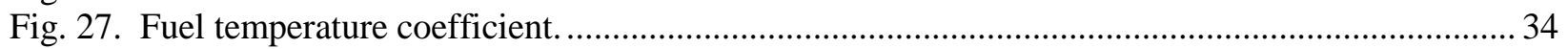

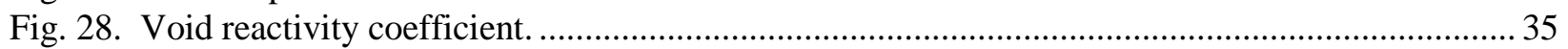

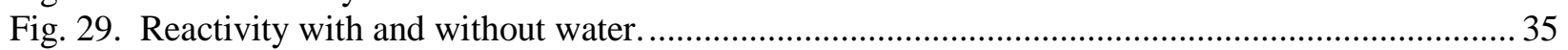

Fig. 30. Cross-sectional view of $12 \times 12$ and $16 \times 16$ fuel assemblies................................................ 43

Fig. 31. Flow chart of MATRA input generator for FCM fuel design. .................................................... 43

Fig. 32. Illustrations of MATRA GUI utility for FCM fuel......................................................... 44

Fig. 33. Pressure drop variation for various rod diameters at different rod arrays. ............................... 46

Fig. 34. Axial distributions of hot channel enthalpy and mass flux for $12 \times 12$ and $16 \times 16$ candidate

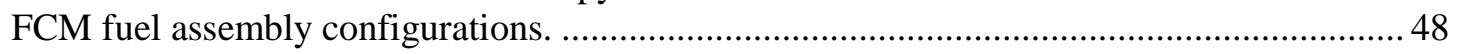

Fig. 35. DNBR and pressure drop on the optimized $12 \times 12$ FCM fuel. ............................................... 49

Fig. 36. DNBR and pressure drop on the optimized 16×16 FCM fuel................................................ 49

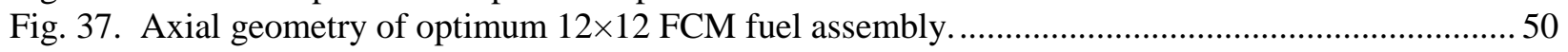

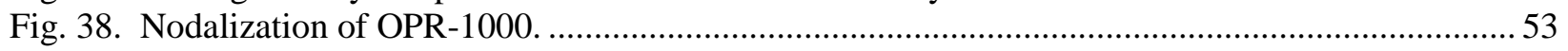

Fig. 39. Radial profile fuel temperature for each fuel type in steady state. ......................................... 57

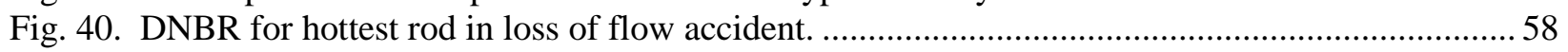

Fig. 41. Cladding temperature for hottest rod in loss of coolant accident. ......................................... 58

Fig. 42. Averaged fuel temperature for hottest rod in rod ejection accident....................................... 59

Fig. 43. Fuel temperature for hottest rod in station blackout............................................................. 60 
Fig. 44. Cladding temperature for hottest rod in LBLOCA without safety injection.

Fig. 45. (a) Secondary electron image of a TRISO particle embedded in the SiC matrix in an FCM pellet; (b) backscattered electron image of TRISO particle matrix SiC interface where the $\mathrm{SiC}$ grain structure in the particle and the matrix are in Snead et al. (2011)

Fig. 46. High-resolution TEM images of (top) SiC nanopowder suspended on a carbon film and (bottom) thin oxide and oxycarbide layer on the surface of the $\mathrm{SiC}$ nanopowder.

Fig. 47. Fractional punch movement and temperature during hot pressing of FCM pellets with surrogate TRISO particles.

Fig. 48. Top: fracture surface of LWR FCM pellet with 44 vol\% TRISO particles and bottom: X-ray radiography of a LWR FCM pellet with $30 \mathrm{vol} \%$ TRISO particles

Fig. 49. Backscattered electron image of $\mathrm{SiC}$ specimens hot pressed at $1850^{\circ} \mathrm{C}$ under $20 \mathrm{MPa}$ of pressure for $1 \mathrm{~h}$. The composition of each specimen is given in Table 18 .

Fig. 50. (a) Bright field image of NITE SiC microstructure with 6 wt. $\%$ yttria-alumina, (b) phase contrast image of a grain boundary in NITE SiC, (c) high-resolution image of the grain boundary between two silicon carbide grains filled with amorphous oxide, (d) phase contrast image of a triple-joint in NITE SiC filled with a crystalline alumina phase.

Fig. 51. (a) Z-contrast, (b) transmission images of NITE SiC microstructure in the scanning TEM mode, and (c) EDS map of oxide additives dispersed between $\mathrm{SiC}$ grains.

Fig. 52. Back-scattered SEM images of FCM fuel with standard $6 \% \mathrm{Y}_{2} \mathrm{O}_{3}$ sintering aid and $1 \%$ $\mathrm{Gd}_{2} \mathrm{O}_{3}$ substitution.

Fig. 53. Plot of reported UN decomposition nitrogen pressure reciprocal temperature data and computed pressures.

Fig. 54. Computed U-C-N phase diagram at $1500 \mathrm{~K}$ at 1 bar total

Fig. 55. Computed nitrogen pressure as a function of temperature over $723-1573 \mathrm{~K}$ and $\mathrm{UC}_{1-\mathrm{x}} \mathrm{N}_{\mathrm{x}}$ composition in equilibrium with carbon or carbides.

Fig. 56. Fraction of a 35 micron SiC layer nitrided per day in a reactor as a function of fuel temperature computed from the relations of Maalmi and Varma.......................................... 75

Fig. 57. Scanning electron microscopy images of SiC exposed to nitriding conditions for 24 hours. ...... 75

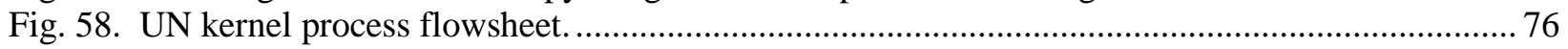

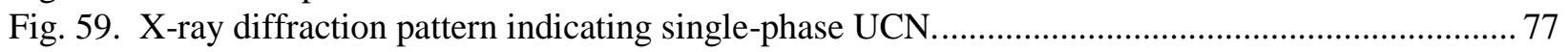

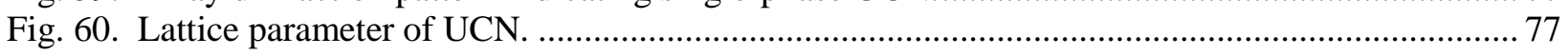

Fig. 61. Swelling data from recent HFIR irradiation of surrogate FCM pellets with a NITE SiC matrix compared to CVD SiC swelling data. ........................................................................ 78

Fig. 62. Calculated, as-irradiated thermal conductivity of as a function of temperature of FCM fuel compared with measured thermal conductivity of $\mathrm{UO}_{2}$ and FCM..................................... 79

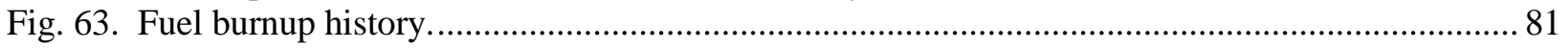

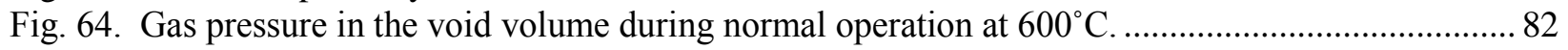

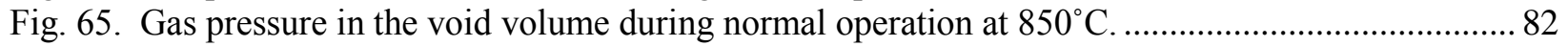

Fig. 66. Gas pressure in the void volume during normal operation at $950^{\circ} \mathrm{C}$. ................................... 83

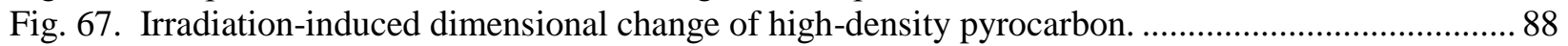

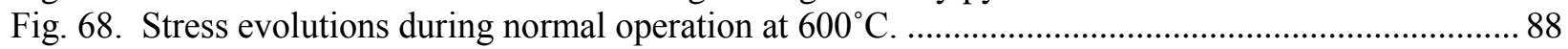

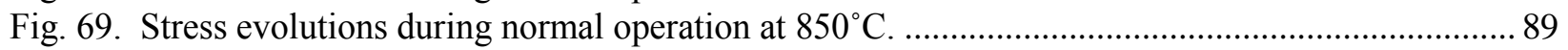

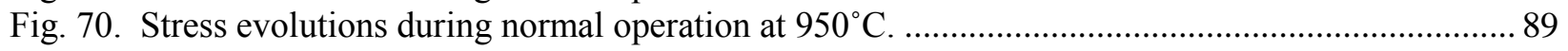

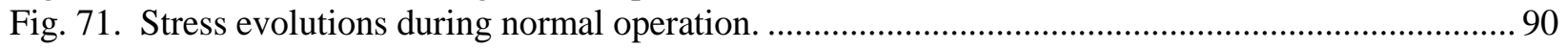

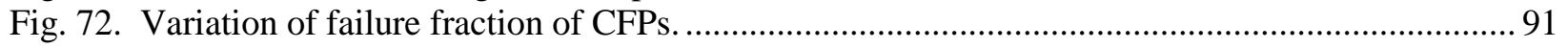

Fig. 73. Stress evolutions in a random CFP during normal operation. ............................................... 91

Fig. 74. FCM fuel array candidates (16×16 conventional, 16×16 FCM, 14×14 FCM, 12×12 FCM fuel array, in order). 


\section{LIST OF TABLES}

\section{Table}

Page

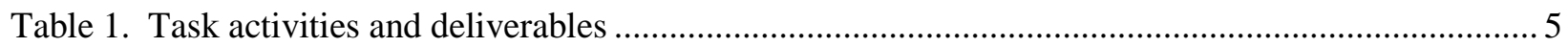

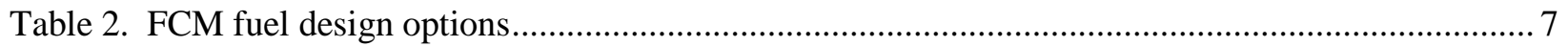

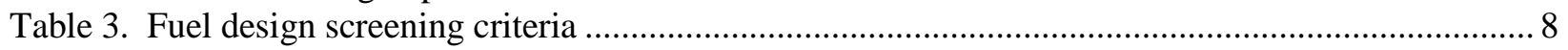

Table 4. Candidate FCM replacement fuel designs for OPR-1000 ................................................... 9

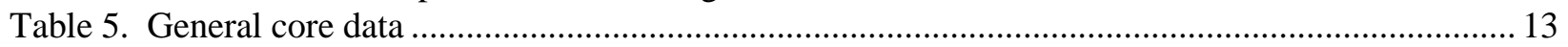

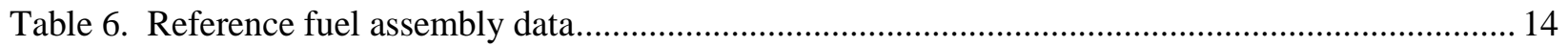

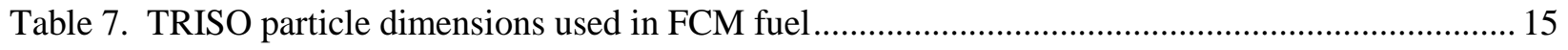

Table 8. Comparison of the heavy metal inventory with kernel diameter .............................................. 16

Table 9. Comparison of the heavy metal inventory with TRISO packing fraction .................................. 17

Table 10. Specifications of fuel rods and fuel assemblies for LWR FCM ........................................... 19

Table 11. MATRA models for scoping analysis of FCM fuel assemblies .......................................... 42

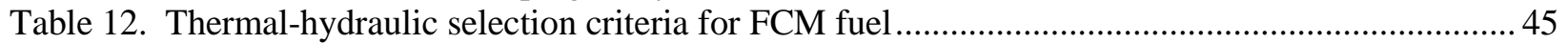

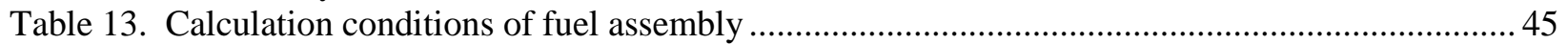

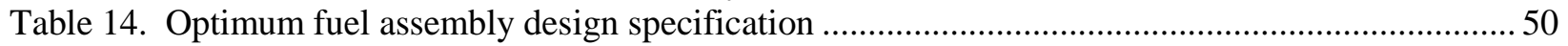

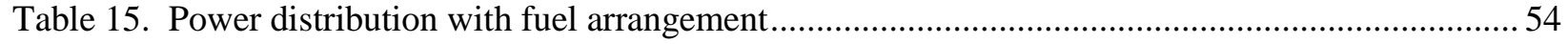

Table 16. Event categorization and acceptance of fuel integrity ........................................................ 55

Table 17. Major parameter of OPR-1000 fueled by the different fuel assembly types ...........................56

Table 18. Composition of various powder mixtures used during hot pressing of monolithic SiC

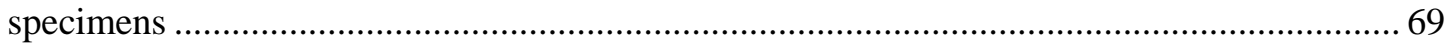

Table 19. Thicknesses and densities of the CFP layers for the FCM-LWR …....................................... 81

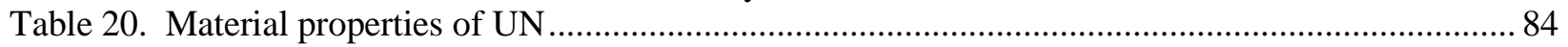

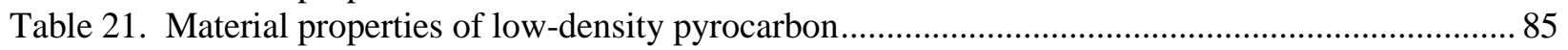

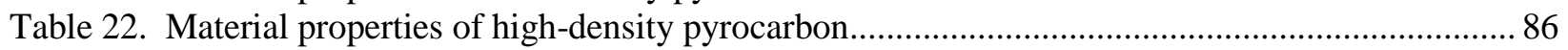

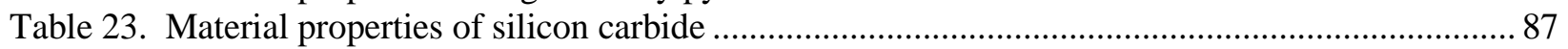

Table 24. Preliminary FCM rod diameters with equivalent fissile inventory ...................................... 93 



\section{LIST OF ACRONYMS}

AOO anticipated operational occurrence

ATWS anticipated transient without scram

BISO bistructural isotropic

BOC beginning of cycle

CFP coated fuel particle

CHF critical heat flux

CMFD coarse-mesh finite difference

CRP coordinated research program

CTR carbothermic reduction

CVD chemical vapor deposited

CVR void reactivity coefficient

DBA design basis accident

DeCART Deterministic Core Analysis based on Ray Tracing

DLL dynamic link library

DNB departure from nucleate boiling

DNBR departure from nucleate boiling Ratio

DOE Department of Energy

EDS energy dispersive X-ray spectroscopy

EFPD effective full power day

EM equal mass exchange

EOC end of cycle

EOS equation(s) of state

FA fuel assembly

FCM fully ceramic microencapsulated

FP fission product

FTC fuel temperature coefficient

GUI Graphic User Interface

HGC Homogenized Group Constants

HFIR High Flux Isotope Reactor

HPSI high-pressure safety injection

HTGR high-temperature gas-cooled reactor

HTP High Thermal Performance

HTR high-temperature reactor 
IAEA International Atomic Energy Agency

IEP isoelectric point

IFM intermediate flow mixer

IIDC irradiation-induced dimensional change

I-NERI International Nuclear Energy Research Initiative

IPyC inner pyrolytic carbon

I/O input/output

KAERI Korea Atomic Energy Research Institute

KD kernel diameter

LEU low-enriched uranium

LOCA loss of coolant accident

LOFA loss of flow accident

LPS liquid-phase sintering

LPSI low-pressure safety injection

LWR light water reactor

MASTER Multi-purpose Analyzer for Static and Transient Effects of Reactor

MATRA Multi-channel Analyzer for steady state and Transients in Rods Array

MDNBR minimum departure from nucleate boiling ratio

MTC moderator temperature coefficient

MOC middle of cycle

Method of Characteristics

MSS-IAS Method of Successive Smoothing with Improved Analytic Solution

MV

Mixing Vane

NGNP Next Generation Nuclear Plant

NITE Nano-Infiltration and Transient Eutectic phase

NPP nuclear power plant

OPR Optimized Power Reactor

OPyC outer pyrolytic carbon

ORNL Oak Ridge National Laboratory

PCMI pellet cladding mechanical interaction

PCT peak cladding temperature

PEI polyethyleneimine

PF packing fraction

PIE post-irradiation examination

PSD particle size distribution 


$\begin{array}{ll}\text { PSG } & \text { Probabilistic Scattering Game } \\ \text { PWR } & \text { pressurized water reactor } \\ \text { PyC } & \text { pyrocarbon } \\ \text { REA } & \text { rod ejection accident } \\ \text { RIA } & \text { reactivity-initiated accident } \\ \text { SA } & \text { severe accident } \\ \text { SAFDL } & \text { specified acceptable fuel design limit } \\ \text { SBO } & \text { station blackout } \\ \text { SENM } & \text { Source Expansion Nodal Method } \\ \text { SiC } & \text { silicon carbide } \\ \text { SS } & \text { stainless steel } \\ \text { TEM } & \text { transmission electron microscopy } \\ \text { TH } & \text { thermal-hydraulics } \\ \text { TPEN } & \text { Triangle-based Polynomial Expansion Nodal } \\ \text { TRISO } & \text { tristructural isotropic } \\ \text { TRU } & \text { transuranic } \\ \text { UCN } & \text { uranium carbonitride } \\ \text { UHP } & \text { ultra-high-purity } \\ \text { UN } & \text { uranium nitride } \\ \text { USNC } & \text { Ultra Safe Nuclear Corporation } \\ \text { WH } & \text { Westinghouse } \\ & \end{array}$





\section{EXECUTIVE SUMMARY}

The objective of the study is to assess the feasibility of replacing the conventional uranium oxide $\left(\mathrm{UO}_{2}\right)$ fuel of the existing fleet of light water reactors (LWRs) with accident-tolerant fully ceramic microencapsulated (FCM) fuel. For feasible fuel replacement, the FCM fuel should behave with comparable levels of power generation and heat transfer as the conventional $\mathrm{UO}_{2}$ fuel throughout the fuel residence in the reactor. To achieve these objectives, a compatible low-enriched uranium (LEU) FCM fuel rod and assembly design should be selected with regards to neutronics, thermal-hydraulics, safety, and fuel performance aspects.

The figure below shows the task structure of the project. For the core analysis, a Korean $1000 \mathrm{MW}$ Optimized Power Reactor (OPR) plant with 16×16 fuel assemblies and a $1200 \mathrm{MW}$ Westinghouse plant with $17 \times 17$ fuel assemblies were selected.

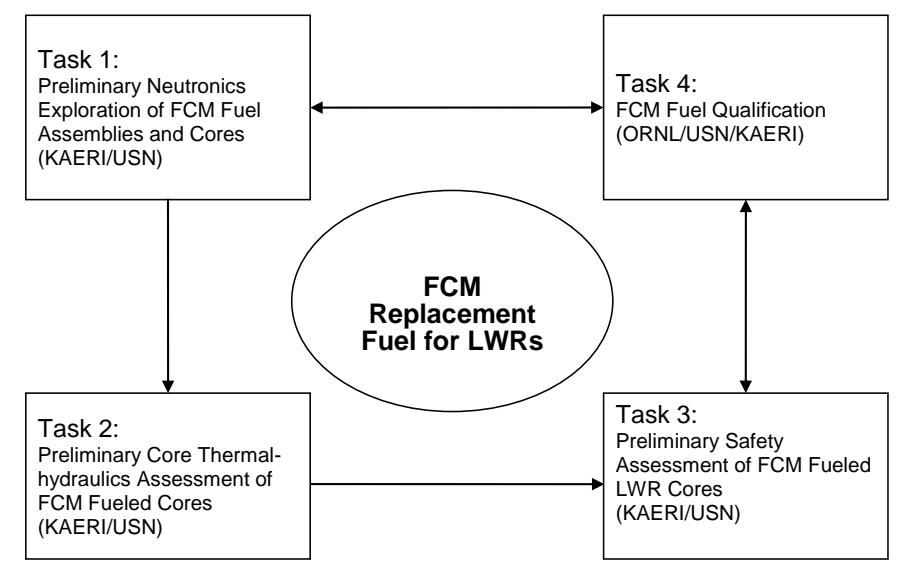

R\&D Task Structure

\section{TASK 1: Preliminary Neutronics Exploration of LWR Fuel Assemblies and Cores}

\section{Brief Description of the Task}

The purpose of this task is to optimize the FCM fuel design, while maintaining compatibility with existing LWR fuel, through neutronics exploration at the fuel assembly level during the first year. In the second year, the static and kinetic core characteristics of the equilibrium core (e.g., power distributions, reactivity parameters, and decay heat) will be generated through core-follow analysis of the full core. The core neutronics parameters generated will be provided as inputs to the thermalhydraulic, safety, and fuel qualification assessment that will follow in the third year.

\section{Task Technical Status Overview}

The code systems established for the FCM fuel assembly and core design analysis are the DeCART2D/MASTER code system for the OPR-1000 core and the SERPENT code for the Westinghouse core. For the OPR-1000 core, fuel assembly depletion calculations have been performed for the FCM-fuel-loaded assemblies, consisting of $12 \times 12$ and $16 \times 16$ grids. Also, the reference solid-fuel-pin-loaded fuel assembly is considered, consisting of a $16 \times 16$ grid. Stainless steel (SS304) and silicon carbide (SiC) cladding materials have been investigated for the uranium nitride (UN)-based FCM fuel. For the Westinghouse core, a $13 \times 13$ configuration is selected. From the scoping analysis using the fuel assembly depletion calculation, the optimized design parameters of the 
fuel assembly have been obtained. By using the optimized fuel assembly design data, the following analyses have been performed: neutron multiplication factor, reactivity coefficients, neutron spectrum effect of the FCM fuel, burnable poison effect, and fast neutron fluence. From the results of the analysis, it is expected that the UN FCM fuel concept can be used in current LWRs; however, full core performance needs to be analyzed in the next year.

\section{Planned Activities}

Task 1 will perform the core analysis of the UN FCM-fuel-loaded OPR-1000 and Westinghouse cores by using the fuel assembly analysis performed this year. The core analysis will provide the cycle-bycycle core reloading performance including the fuel assembly loading patterns, cycle length, and reactivity coefficients. Also, an accurate determination of the fast neutron fluence will be performed.

\section{Issues/Concerns}

The first issue within Task 1 is that the fast neutron fluence of the FCM fuel, as determined in the constant power assembly level analysis, appears to be slightly higher than that of the regular oxide fuel in the reference LWR and significantly higher than that of tristructural-isotropic (TRISO) fuel particles in high-temperature reactors (HTRs). The increased fluence will affect TRISO fuel performance and the core cycle length. A more realistic and accurate determination of the fluence, taking into account the age and power level of the fuel, will be performed next year in the course of the full core reload analysis. A second issue is the positive moderator temperature coefficient observed with high soluble boron concentration in the water coolant. It is reasonably expected that this issue will be resolved by the use of burnable poison during the core reload analysis.

\section{TASK 2: Preliminary Core Thermal-Hydraulics Assessment of FCM-Fueled LWR Cores}

\section{Brief Description of the Task}

The thermal-hydraulic analysis is performed to demonstrate the thermal-hydraulic and safety compatibility of the FCM fuel in the existing LWR cores. Thermal and safety margins to the departure from nucleate boiling (DNB) are optimized by assessing the heat transport capability under normal, transient, and accident conditions.

\section{Task Technical Status Overview}

Subchannel analysis codes developed for pressurized water reactors (PWRs) and advanced reactor cores are evaluated for applicability to the thermal-hydraulic analysis of the FCM-loaded core. Refinement of the pertinent thermal-hydraulic models for the assembly pressure drop and departure from nucleate boiling ratio (DNBR) analysis is required for modeling the FCM fuel with various spacers and pitch-to-diameter ratios. Using the current analysis code and models, preliminary scoping analysis for the assembly pressure drop and DNBR has been performed for OPR-1000 16×16 and $12 \times 12$ fuel geometries with different pitch-to-diameter ratios. Based on the analysis, feasible fuel assembly designs that satisfy the screening criteria are proposed.

\section{Planned Activities}

Major efforts in the first year for Task 2 consist of (1) establishing the core thermal-hydraulic analysis system and (2) core/subchannel modeling and preliminary analysis. The thermal-hydraulic analysis system will be established on the basis of a subchannel analysis code, empirical models, and a utility program for a graphical user interface (GUI). Preliminary analysis results on the optimum geometry 
of FCM fuel will be provided by conducting a systematic evaluation on the pressure drop and DNBR in fuel assemblies.

\section{Issues/Concerns}

The main issue of Task 2 is the thermal-hydraulic compatibility of FCM fuel with the existing OPR-1000 fuel assembly. In the first year, a core fully fueled with FCM fuel was evaluated to have sufficient thermal margin to ensure the safety of the OPR-1000; however, in the transition core, newly loaded FCM fuel assemblies that have a higher pressure drop cannot deteriorate the thermal margin of the existing fuel assemblies outside of the acceptance limit. To achieve this, a high thermal performance design is required for the FCM fuel in order to compensate for the reduced thermal margin caused by a flow reduction. A spacer grid with a high thermal performance and small pressure drop is essential to resolve this issue.

\section{TASK 3: Preliminary Safety Assessment of FCM-Fueled LWR Cores}

\section{Brief Description of the Task}

The design safety of FCM-fueled cores is assessed for the limiting design basis accident (DBA) scenarios: loss of flow accidents (LOFAs) for departure from nucleate boiling ratio (DNBR) margin, and loss of coolant accidents (LOCAs) for peak cladding temperature (PCT) margin. In addition, the accident analysis for beyond DBA (BDBA) scenarios, such as station blackout (SBO) and multiple safety system failure, is carried out to demonstrate the accident-tolerant envelope of the FCM fuel.

\section{Task Technical Status Overview}

MARS-MASTER coupled safety analysis methodology has been developed for DBA and BDBA analysis. In order to assess the FCM-fueled cores, limiting accident scenarios are analyzed, where preliminary safety criteria applicable to the FCM fuel are applied. An OPR-1000 fueled with FCM fuel is modeled using the MARS code, and the preliminary safety analyses are performed for limiting DBA scenarios for LOFAs, LOCAs, and rod ejection accidents (REAs) and for extreme scenarios such as without safety injection (SI) and SBO. From the safety analysis, it is found that the safety of FCM-fueled cores is ensured with sufficient margin.

\section{Planned Activities}

Limiting safety analysis for the transition cores will be carried out in the second project year. For this activity, transition core models based on core geometry and core physics parameters will be developed. For REA scenarios where strong feedback from the core kinetics plays an important role, MARS/MASTER coupled analysis will be performed. In addition, a radiation heat transfer model will be developed for realistic simulation of BDBA scenarios.

\section{Issues/Concerns}

Safety criteria of the new FCM fuel and SiC cladding have not been well quantified up to now. In addition, it is found that $\mathrm{SiC}$ properties after irradiation are key parameters affecting the initial fuel temperature distribution, initial stored energy, and resulting accident consequence. In the first project year, we assumed criteria and properties based on available material data. It is recommended that experiments to quantify the safety-related criteria and material properties should be performed in the longer term for further development and potential commercialization. 


\section{TASK 4: FCM Fuel Qualification}

\section{Brief Description of Objectives}

FCM fuel is currently under development as a replacement fuel for $\mathrm{UO}_{2}$ in commercial LWRs. As such, this program addresses key technical issues in the development path of that fuel system, and this task specifically identifies fabrication and material/fuel performance issues. In this task, fuel optimization and targeted irradiation studies will be carried out. A computer model to assess the FCM fuel behavior is to be set up for analytic prediction of fuel performance.

\section{Task Technical Status Overview}

In the first year of this International Nuclear Energy Research Initiative (I-NERI), and in parallel with ongoing activities to develop the FCM fuel, significant progress has been made in (1) overcoating of fuel to address specific fabrication issues, (2) optimization of the FCM matrix for density and thermal conductivity, (3) successful demonstration of a route to high-density UN kernel fabrication, and (4) positive results from the first series of irradiation capsules targeting the stability of the FCM matrix material and the investigation of fuel/clad interaction.

A TRISO particle fuel performance analysis code has been developed and verified through benchmark calculations. The material properties have been obtained as relevant under HTR conditions. The design technology of a dual-cooled annular fuel for existing LWRs was developed. This will contribute to the design of the FCM LWR fuel.

\section{Planned Activities}

Activities will continue as planned including (1) continued FCM process development and properties investigation, (2) scaling the UN kernel fabrication to batch quantities leading to TRISO coating and compaction, and (3) irradiation of fueled FCM.

The FCM fuel performance evaluation activities consist of (1) estimation of fuel burnup and depletion, (2) analysis of gas pressure buildup in the void volume of the kernel and buffer of the coated fuel particle (CFP), (3) thermo-mechanical analysis of the CFP, (4) estimation of the failure fraction of a CFP batch, (5) analysis of fission product transport from the CFPs, through pellet and cladding into the coolant, (6) thermo-mechanical analysis of pellet and cladding, (7) fuel rod thickness determination, disposition, and overall rod dimensioning in the fuel assembly, (8) flow-induced vibration analysis for transition core (where reference oxide and FCM fuel may be present at the same time).

\section{Issues/Concerns}

Thus far, the irradiation studies indicate that the matrix is quite stable at the conditions studied and there is no fuel-clad interaction; however, near-term concerns to be considered include: (1) the FCM process lends itself to intermediate- to large-part-scale fabrication but small cylinder fabrication is challenging; (2) UN kernel development, while initially encouraging, is still a development program and needs to be demonstrated at batch scale and beyond; (3) the irradiation stability of UN at LWR relevant temperatures; and (4) funding for such aggressive concerns is an issue.

The behavior of the FCM fuel material is not well known under LWR operating conditions. It is necessary to secure new material properties and irradiation performance data produced under extended fluence. 


\section{OVERVIEW OF THE PROJECT}

\subsection{PROJECT DESCRIPTION}

The Fukushima Nuclear Incident has demonstrated the vulnerability of $\mathrm{UO}_{2}$ fuels in light water reactors (LWRs) to becoming severely damaged during Station Blackout (SBO) events. The tristructural-isotropic (TRISO)-based fully ceramic microencapsulated (FCM) fuel recently conceived by Oak Ridge National Laboratory (ORNL) and Ultra Safe Nuclear Corporation (USNC) scientists, and under development at ORNL, is a promising medium-term concept (10-15 years) to replace current $\mathrm{UO}_{2}$ fuel pellets. FCM fuel has potentially superior safety characteristics relative to other fuel forms as a result of its multiple barriers to fission product dispersion, high mechanical stability, and good thermal conductivity. However, the low fissile density caused by the presence of these barriers in the fuel needs to be compensated by appropriate fuel and core design. Additionally, the neutronic and thermal-hydraulic compatibility with existing LWR cores should be verified for practical application.

A 3-year International Nuclear Energy Research Initiative (I-NERI) collaboration is being carried out through a collaborative effort among ORNL, USNC, and the Korea Atomic Energy Research Institute (KAERI) assessing the feasibility of replacing the current fuel of the existing fleet of LWRs with FCM fuel. The objective of the project is to select a suitable low-enriched uranium (LEU) FCM fuel rod and assembly design and to assess the feasibility of replacing the conventional uranium oxide $\left(\mathrm{UO}_{2}\right)$ fuel with accident-tolerant FCM fuel in a representative sample of the existing LWR fleet. For feasible fuel replacement, the FCM fuel should exhibit comparable levels of power generation and heat transfer as the conventional $\mathrm{UO}_{2}$ fuel throughout the fuel operational life. To achieve the objective, a compatible LEU FCM fuel rod and assembly design should be selected with regards to neutronics and thermal-hydraulics aspects. The thermal-hydraulic and safety performance of FCM fuel should be confirmed under operational transients and accident conditions, including design basis accident (DBA) and beyond design basis accident (BDBA) events. Moreover, the fuel mechanical performance under irradiated conditions should be properly qualified, that is, accurately described analytically and confirmed experimentally. The analytical tools and methods applied and the fuel qualification data obtained experimentally will form the basis for a future licensing application.

This R\&D plan covers preliminary assessment of the compatibility of FCM fuel assemblies in existing LWR cores, as well as FCM fuel qualification in realistic LWR environments. The four tasks and their interrelationship are illustrated below. The reference cores selected are a Westinghouse (WH) core with $17 \times 17$ fuel assemblies and a Korean OPR-1000 core with $16 \times 16$ fuel assemblies. USNC takes the lead in the WH plant application, while KAERI takes the lead in the OPR-1000 application. USNC and KAERI cooperate in the analysis of the results. ORNL takes the lead in all the experimental tasks.

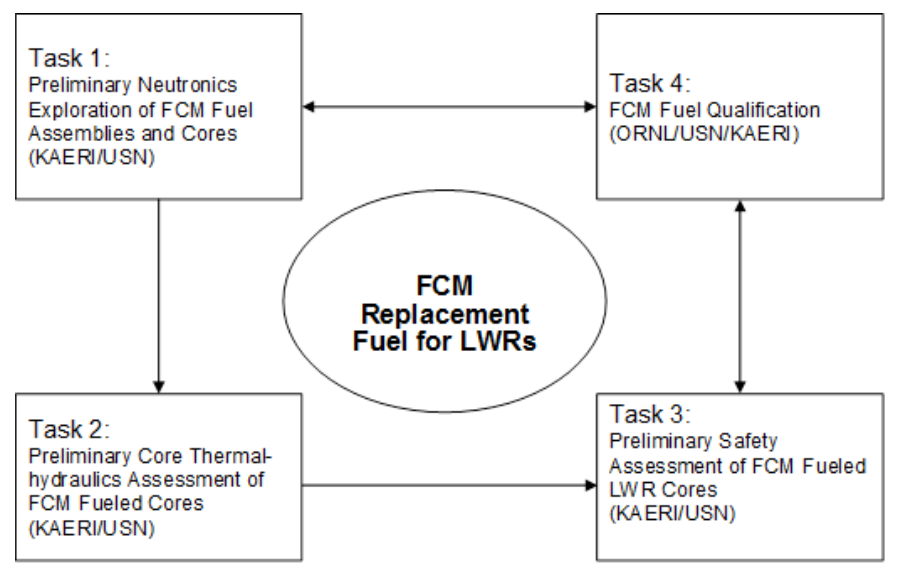




\subsection{BACKGROUND}

The TRISO particle fuel, historically developed through the various high-temperature gas-cooled reactor (HTGR) programs and recently requalified by the Department of Energy Next Generation Nuclear Plant (DOE-NGNP) program, shows excellent performance in radioactivity retention capability through the presence of multiple layers of ceramic coating, which are chemically stable and mechanically strong at very high temperature and very high burnup.

The FCM fuel has been successfully manufactured by replacing the well-established carbon-based matrix of traditional TRISO fuel compacts with a high-density silicon carbide (SiC) matrix. In addition to being thermally conductive and radiation tolerant, the dense $\mathrm{SiC}$ matrix provides a substantial barrier to fission product dispersion, augmenting the multiple barriers of TRISO. Therefore, extremely high radioactivity retention capability is expected, even with regard to fission products that are mobile in the HTGR TRISO environment, as well as a much lower production of carbon-14 (typically induced by graphite porosity). Furthermore, the good thermal conductivity and heat capacity of $\mathrm{SiC}$ (relative to solid oxide fuels) dramatically reduces the peak fuel temperature as well. The low swelling of $\mathrm{SiC}$ under irradiation and no release of gaseous fission products from the fuel should reduce the size and/or need of a fuel/clad gap and plenum. Finally, the replacement of zirconium-alloy cladding material with alternate stainless steel or SiC should reduce or eliminate the potential for hydrogen explosion due to rapid clad/steam reaction.

The Fukushima nuclear disaster of March 11, 2011 has revealed a significant vulnerability of $\mathrm{LWR} \mathrm{UO}_{2}$ fuels in that the fuel rods can be severely damaged during SBO events. Even with various engineered safety features, the adequate removal of decay heat was not possible because of the extended unavailability of electric power. As a consequence, cladding damage and fuel meltdown could not be avoided, and the hydrogen explosion resulting from the zirconium cladding oxidation caused massive radioactivity release to the environment.

The fuel rods made by FCM fuel compacts and alternate cladding material, which is very similar in shape to the $\mathrm{UO}_{2}$ pellets, can be used in current LWRs without large modification of the reactor or the plant. The replacement of fuel will increase the accident tolerance of the existing fleet of LWRs and advanced LWRs by (1) improving reaction kinetics with steam, (2) reducing the hydrogen generation rate, (3) improving fuel and cladding thermo-mechanical properties, and (4) improving fission product retention. Due to the low fissile density of TRISO fuel, however, a suitable fuel design should be found in order to achieve comparable neutronic, thermal-hydraulic, and safety performance with existing LWR fuel assemblies.

\subsection{TASK DESCRIPTION}

The objective of the proposed study is to assess the feasibility of using FCM fuel assemblies in place of conventional LWR fuel assemblies with minimal modification to the reactor systems or the operations. By necessity, the compatible FCM fuel must have similar fissile content, in order to produce as much fission power as the existing LWR fuel (on a unit assembly basis). It is possible to increase the uranium enrichment from approximately 5\%, typical of current LWR fuel, up to the nonproliferation limit of $20 \%$, but ideally it would be desirable to stay as close as possible to current enrichment levels. Therefore it is preferable to try to increase the volume available to the fuel and the density of the fuel, before increasing the enrichment. The volume of the fuel can be augmented by increasing the fuel rod diameter. Other options include optimizing the coating layer thickness to the LWR burnup and power density envelope; increasing the size of the TRISO particles and packing fraction to provide a larger initial fissile inventory; and using higher fissile density kernels such as uranium nitride, uranium carbide, and uranium silicide. The stainless steel and $\mathrm{SiC}$ options for cladding will significantly reduce the hydrogen generation issue. 


\subsubsection{Task 1: Preliminary Neutronics Exploration of FCM Fuel Assemblies and Cores}

The neutronics analysis of the fuel assembly is the starting point of the feasibility study. The FCM fuel design compatible with existing LWR fuel is optimized through the neutronics exploration of the fuel assembly design. Then, the static and kinetic core characteristics of the equilibrium core, such as power distributions, reactivity parameters and decay heat, etc., are generated through the full core-follow analysis. The core neutronics parameters generated throughout the analysis are provided as inputs to the thermal-hydraulic, safety, and fuel qualification assessments. Task 1 consists of the following subtasks:

- $\quad$ LEU FCM fuel rod design containing $\mathrm{UO}_{2}, \mathrm{UCO}, \mathrm{UC}$, or UN TRISO particles in SiC matrix;

- $\quad$ LEU FCM fuel assembly design that can replace existing LWR core, taking into account the k-infinity, in-core cycle length and core peaking;

- transition core loading pattern search for reference FCM LWR core, taking into account the core physics and safety parameters; and

- equilibrium core search for reference LWR core, taking into account the fuel loading pattern, core physics and safety parameters.

KAERI will work on core configurations specific to the Korean OPR-1000 reactor $(16 \times 16$ fuel assemblies). USNC will work on core configurations specific to the US Westinghouse reactor $(17 \times 17$ fuel assemblies). The outcome of this preliminary study will provide guidance for further optimization. Subtasks shall include the computation of core physics and safety parameters such as power distributions, shutdown margins, kinetic parameters such as reactivity coefficients for fuel and moderator at the beginning of cycle (BOC), middle of cycle (MOC), and end of cycle (EOC) for use in the core thermalhydraulics and safety assessments.

\subsubsection{Task 2: Preliminary Core Thermal-Hydraulics Assessment of FCM-Fueled LWR Cores}

The thermal-hydraulic analysis of the fuel assembly is required to demonstrate safety and compatibility of the FCM fuel for the existing LWR cores. The thermal and safety margins to the DNB are confirmed by assessing the heat transport capability under normal, transient and accident conditions. Fuel pressure drop analysis is used to assess the core flow distribution and the fuel lift-up force. The assessment is performed at the core, fuel assembly, and subchannel levels. Task 2 consists of the following subtasks:

- $\quad$ subchannel modeling of reference LEU FCM fuel assembly and core;

- $\quad$ scoping studies of the FCM fuel assembly and subchannel thermal-hydraulics including pressure drop and thermal margin to DNB for a reference core $\left(1^{\text {st }}\right.$ cycle) obtained from Task 1 ; and

- core-wise to subchannel thermal-hydraulics assessment on the thermal and safety margins for a reference core (equilibrium) obtained from Task 1.

KAERI will work on the OPR-1000 $16 \times 16$ assembly core configuration. USNC will work on the Westinghouse $17 \times 17$ assembly core configuration.

\subsubsection{Task 3: Preliminary Safety Assessment of FCM Fueled LWR Cores}

The safety of the FCM fuel is finally assessed and confirmed by the system-level accident analysis. Design safety margins are quantified for limiting DBA scenarios: the loss of flow accidents for the DNB margin, and the loss of coolant accidents for the peak cladding temperature (PCT) margin. In addition, the accident analysis for the BDBA scenarios such as SBO and multiple safety system failures will attempt to demonstrate the accident-tolerant envelope of the FCM fuel. Task 3 consists of the following subtasks: 
- $\quad$ modeling of the reference reactor system with LEU FCM core including evaluation of the impact of FCM fuel replacement on the reactor system thermal-hydraulics (pumps, steam generators)

- $\quad$ scoping studies on the safety performance of the reference reactor system for selected DBA and BDBA scenarios using the core physics and safety parameters (1st cycle) obtained from Task 1, and

- $\quad$ system-level accident analysis for selected DBA and BDBA scenarios using the core physics and safety parameters (equilibrium core) obtained from Task 1 and 2.

KAERI will work on the OPR-1000 $16 \times 16$ assembly core configuration. USNC will work on the Westinghouse $17 \times 17$ assembly core configuration.

\subsubsection{Task 3: FCM Fuel Qualification}

Experimental qualification of the FCM fuel performance by irradiation and post-irradiation examination and subsequent iterations in fabrication recipes and manufacturing techniques are required to confirm the FCM fuel performance within the envelope of DBAs as well as normal operations. A computer model to assess the FCM fuel behavior should also be set up for analytic prediction of performance for future application of the FCM fuel to LWRs. Task 4 consists of the following subtasks:

- manufacturing of FCM fuel samples (using both surrogate kernels and uranium kernels);

- $\quad$ irradiation of FCM fuel samples;

- post-irradiation examination of FCM fuel;

- performance modeling of reference LEU FCM fuels (TRISO models and LWR fuel/clad/coolant models); and

- fuel performance analysis during accident (using equilibrium core conditions).

Manufacturing, irradiation, and post-irradiation examination of the FCM fuel will be performed at ORNL. KAERI and USNC will produce a practical model of fuel performance for the FCM fuel, integrating existing TRISO fuel models with LWR fuel/clad and clad/coolant models.

\subsection{PROJECT SCHEDULE AND MILESTONES}

Each of the main tasks described in Section 1.3 is scheduled to span the 3-year duration of the project. Table 1 shows the subtasks comprising the four main tasks along with the lead organization and milestone completion date. 
Table 1. Task activities and deliverables

\begin{tabular}{|c|l|c|c|}
\hline $\begin{array}{c}\text { Task } \\
\text { number }\end{array}$ & \multicolumn{1}{|c|}{ Task activity description } & Lead organization & $\begin{array}{c}\text { Milestone } \\
\text { date }\end{array}$ \\
\hline 0.0 & Project Management & ORNL/USNC/KAERI & \\
\hline 0.1 & Annual Progress Reports & ORNL/USNC/KAERI & Annual \\
\hline $\mathbf{1 . 0}$ & Task 1. Preliminary Neutronics Exploration & KAERI/USNC & \\
\hline 1.1 & LEU FCM fuel rod design & KAERI/USNC & 12 months \\
\hline 1.2 & LEU FCM fuel assembly design & KAERI/USNC & 12 months \\
\hline 1.3 & Transition core loading pattern search & KAERI/USNC & 24 months \\
\hline 1.4 & Equilibrium loading pattern and final neutronics design & KAERI/USNC & 36 months \\
\hline $\mathbf{2 . 0}$ & Task 2: Preliminary Core Thermal-Hydraulics (TH) & KAERI/USNC & \\
\hline 2.1 & Assessment & KAERI/USNC & 12 months \\
\hline 2.2 & Scoping studies & KAERI/USNC & 24 months \\
\hline 2.3 & Final thermal-hydraulics assessment & KAERI/USNC & 36 months \\
\hline $\mathbf{3 . 0}$ & Task 3. Preliminary Safety Assessment & KAERI/USNC & \\
\hline 3.1 & Modeling of reference reactor systems & KAERI/USNC & 12 months \\
\hline 3.2 & Scoping studies & KAERI/USNC & 24 months \\
\hline 3.3 & System-level accident analysis & KAERI/USNC & 36 months \\
\hline $\mathbf{4 . 0}$ & Task 4. FCM Fuel Qualification & ORNL & \\
\hline 4.1 & Manufacturing FCM fuel sample & ORNL & 12 months \\
\hline 4.2 & Irradiation of FCM fuel & ORNL & 24 months \\
\hline 4.3 & Post-irradiation examination (PIE) of FCM fuel & ORNL & 36 months \\
\hline 4.4 & Performance modeling of FCM & 24 months \\
\hline 4.5 & Performance analysis during accident & 36 months \\
\hline & & & \\
\hline
\end{tabular}




\section{FCM FUEL DESIGN CONCEPT}

The Fukushima accident triggered the need to develop a meltdown-resistant and accident-tolerant fuel that does not lead to severe fuel failure and subsequent hydrogen release during extreme accident conditions, such as an SBO event. The "accident tolerance" of the fuel can be enhanced by lower fuel operating temperature, higher fuel damage temperature, strong mechanical integrity at temperatures higher than operational conditions, reduced hydrogen generation, improved fission product retention, and increased resistance to oxidation. For enhanced accident tolerance, an innovative fuel concept, FCM fuel, is proposed to replace the conventional $\mathrm{UO}_{2}$ fuel for existing and advanced LWRs.

The FCM fuel pellet consists of TRISO particle fuel highly packed in a dense SiC matrix, with stainless steel (SS) or SiC replacing conventional zirconium-alloy cladding, as shown in Figure 1. The accidenttolerant features of the FCM fuel can be summarized as follows.

- Resistance to meltdown, provided by the use of refractory SiC-layered TRISO particles and the SiC matrix with a melting temperature around $2400^{\circ} \mathrm{C}$

- Resistance to radioactivity release, ensured primarily by the TRISO particle and additionally by the $\mathrm{SiC}$ pellet matrix (the indefinite fission product primary retention capability of the TRISO particle up to $1600^{\circ} \mathrm{C}$ is well proven from the gas-cooled reactor experiences)

- Resistance to fuel thermo-mechanical degradation, provided by the heat-conductive $\mathrm{SiC}$ pellet, which lowers the fuel centerline temperature and temperature gradient during normal and accident conditions. In addition, the fission product retention by the TRISO particles and $\mathrm{SiC}$ matrix reduces the fuel rod internal pressure and subsequent mechanical stress to the cladding

- Resistance to hydrogen production, provided by the use of SS or SiC as cladding materials, which have slower reaction kinetics with steam and, thus, a slower hydrogen generation rate than conventional zirconium-alloy cladding

- Resistance to nuclear proliferation, maintained by the complexity of plutonium extraction from the spent FCM fuel pellet and TRISO particles and by its low quality for conversion to nuclear weapon materials

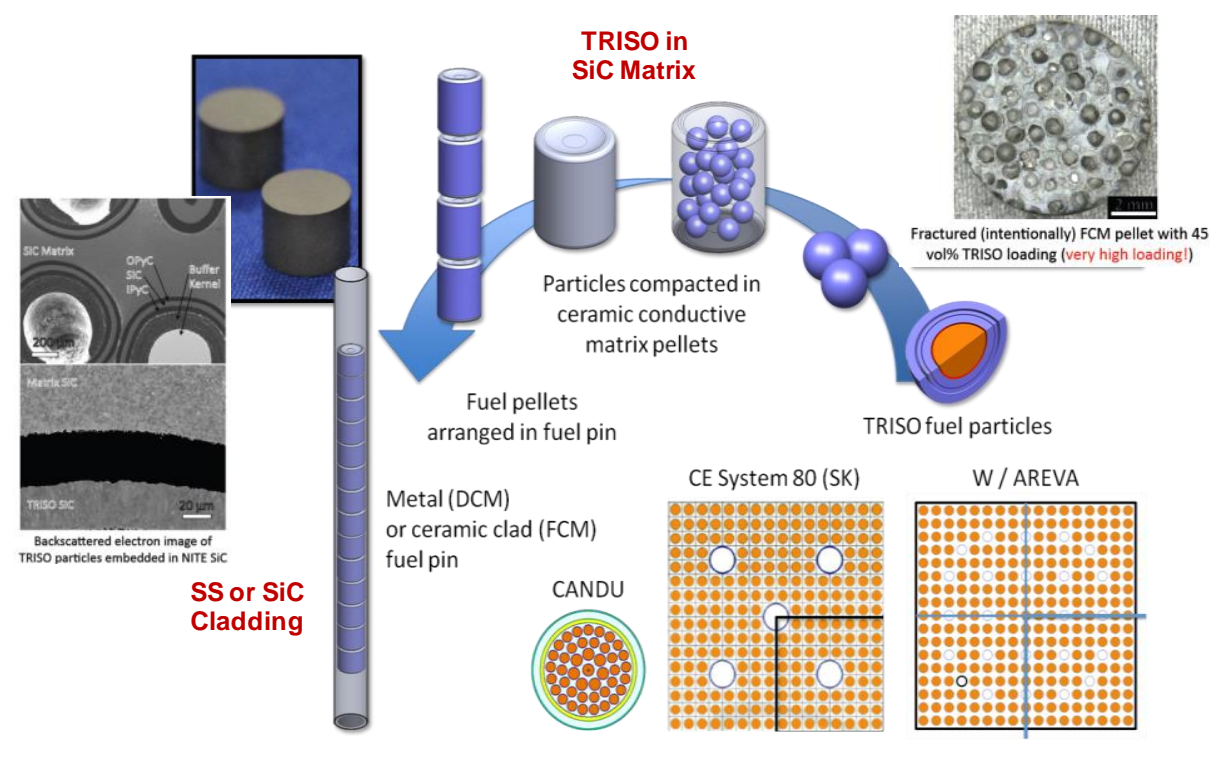

Fig. 1. FCM fuel design concept. 
In order to identify a feasible replacement fuel, the candidate FCM fuel designs are screened to be compatible with existing Korean standard $16 \times 16$ and Westinghouse $17 \times 17$ fuel assemblies and cores. Due to the low initial fissile density of the FCM fuel pellet consisting of TRISO particles and SiC matrix, the neutronic compatibility of the FCM fuel designs is first determined for various fissile materials (e.g., UC, UCO, and UN), fuel enrichments, TRISO packing fractions, and fuel rod dimensions by checking for similar core reactivity $\left(k_{\text {eff }}\right)$, cycle length, and reactivity coefficients relative to the existing reference cores. The replacement fuel assembly designs are $12 \times 12$ or $16 \times 16$ arrays for the Korean standard reactors, OPR-1000, and $13 \times 13$ or $17 \times 17$ arrays for the $\mathrm{WH}$ reactors. These fuel assembly arrays are selected to meet the mechanical compatibility with internal structures and control mechanisms of the existing cores, while satisfying the neutronic and thermal-hydraulic compatibility. The thermal-hydraulic compatibility of the candidate designs is examined for their pressure drop, DNB, and cross flow characteristics. The TRISO fuel integrity is evaluated through fuel performance analysis throughout the core lifetime. Finally, the safety of the FCM fueled core is assessed for limiting DBAs such as loss of flow accident (LOFA), loss of coolant accident (LOCA), reactivity-initiated accident (RIA), and for extreme BDBA events such as LOCA without safety injection and extended SBO accidents. The screening process just described is iterated until feasible FCM fuel designs are obtained.

In this first year, the fuel rod and assembly level analyses have been performed and the feasible FCM fuel designs are now proposed for the core level analysis in the next year. In parallel, FCM fuel samples have been manufactured, irradiated, and tested for material properties and integrity.

\subsection{FCM FUEL DESIGN OPTIONS}

Table 2 lists several fuel design options reviewed for the FCM fuel designs. Options I and II are selected for quantitative evaluation since they are based on current and state-of-the-art available manufacturing and design technologies. Option III introduces an innovative monolithic concept in which a SiC fuel element with TRISO particles is manufactured and sintered as a solid rod. This option requires more study with respect to its manufacturability, so that only the accident tolerance during extreme events is evaluated, for future possible implementation. Other options were reviewed but ultimately screened out, such as the collapsible cladding concept (used in heavy water reactor fuel) and the dispersed TRISO without $\mathrm{SiC}$ matrix in a freestanding cladding.

Table 2. FCM fuel design options

\begin{tabular}{|c|c|c|c|c|c|}
\hline Option & Particle fuel & Pellet matrix & Gap & Cladding & Remarks \\
\hline I & TRISO & $\mathrm{SiC}$ & $\mathrm{He}$ & SS Freestanding & Full evaluation \\
\hline II & TRISO & $\mathrm{SiC}$ & $\mathrm{He}$ & $\begin{array}{c}\text { SiC Triplex } \\
\text { Freestanding }\end{array}$ & Full evaluation \\
\hline III & TRISO & $\mathrm{SiC}$ & (none) & SiC Monolithic & Accident evaluation \\
\hline
\end{tabular}

\subsection{DESIGN SCREENING CRITERIA}

The initial FCM fuel design screening criteria, summarized in Table 3, were established prior to quantitative analysis. They are imposed to maintain the neutronic, thermal-hydraulic, and mechanical compatibility with existing core and fuel designs, while meeting current manufacturing capability. 
Table 3. Fuel design screening criteria

\begin{tabular}{|c|c|c|c|}
\hline Component & Design parameter & Design ranges & Design consideration $^{a}$ \\
\hline \multirow{5}{*}{ Fuel Pellet } & Fissile Material & $\mathbf{U O}_{2}, \mathbf{U C O}, \mathbf{U N}$ & $\mathbf{N C}$ \\
\hline & Kernel Diameter & $500-700 \mu \mathrm{m}$ & NC, FP, M \\
\hline & ${ }^{235} \mathrm{U}$ Enrichment & $<20 \mathrm{w} / \mathrm{o}$ & NC \\
\hline & Pellet Diameter & Relates to fuel diameter & NC, TH \\
\hline & TRISO Packing Fraction & $\mathbf{4 0}-\mathbf{5 8 \%}$ & $\mathrm{NC}, \mathrm{M}$ \\
\hline \multirow{5}{*}{ Fuel Rod } & Cladding Thickness (SS) & $>0.59 \mathrm{~mm}$ & MC, $\mathbf{M}$ \\
\hline & Cladding Thickness (SiC) & $>1 \mathrm{~mm}$ & $\mathrm{MC}, \mathbf{M}$ \\
\hline & Fuel Rod Diameter & $10.75-15.9 \mathrm{~mm}$ & MC \\
\hline & Pellet-Cladding Gap (He) & $\sim$ Ref. $^{b}$ & $\mathbf{M}$ \\
\hline & Initial Gap Pressure & $<8 \mathrm{MPa}$ & MC \\
\hline \multirow{10}{*}{ Fuel Assembly } & FA Array (OPR-1000 16×16) & $12 \times 12$ or $16 \times 16$ & $\mathrm{NC}, \mathrm{MC}$ \\
\hline & FA Array (WH 17×17) & $13 \times 13$ & $\mathrm{NC}, \mathrm{MC}$ \\
\hline & Pressure Drop/Lift Force & Ref. $<120 \%$ & TH, MC \\
\hline & Cross Flow & $<1 \mathrm{~m} / \mathrm{s}$ & TH \\
\hline & Spacer Grid Design & Proven & TH \\
\hline & Guide Tube & Single/Double & MC \\
\hline & DNBR & $\sim$ Ref. & $\mathbf{T H}$ \\
\hline & Gap between Fuel Rods & $>2 \mathrm{~mm}$ & MC \\
\hline & Fuel Cycle Length & Ref. (18 months) & NC \\
\hline & Core Kinetics Parameters & $\sim$ Ref. & $\mathbf{N C}, \mathbf{S A}$ \\
\hline
\end{tabular}

${ }^{a} \mathrm{NC}$ : neutronic compatibility, TH: thermal-hydraulic compatibility, MC: mechanical compatibility, FP: fuel performance, SA: Safety Analysis, M: manufacturability

${ }^{b}$ Ref.: Reference value of OPR-1000 and WH 


\subsection{CANDIDATE DESIGNS}

\subsubsection{FCM Replacement Fuel for OPR-1000 Core}

After iterating the scoping analysis for neutronic and thermal-hydraulic compatibility within the various parameter combinations proposed in the design screening criteria, four candidate FCM replacement fuel designs for OPR-1000 were proposed: SS or SiC cladding fuel rod designs in $12 \times 12$ or 16×16 fuel assembly arrays. Table 4 summarizes and compares the candidate designs with the original $\mathrm{UO}_{2}$ fuel design. For these candidate designs, quantitative analyses were performed for the core neutronic, thermalhydraulic, fuel thermo-mechanical, and safety performance. Detailed scoping and design analysis results are given in the following sections.

Table 4. Candidate FCM replacement fuel designs for OPR-1000

\begin{tabular}{|c|c|c|c|c|c|c|}
\hline \multirow[t]{2}{*}{ Parameters } & \multirow{2}{*}{$\begin{array}{c}\text { Unit } \\
\mathrm{cm}\end{array}$} & \multicolumn{3}{|c|}{ Original } & \multicolumn{2}{|c|}{ FCM fuel assembly } \\
\hline & & $16 \times 16$ solid & $12 \times 12-\mathrm{SS}$ & $12 \times 12-\mathrm{SiC}$ & $16 \times 16-\mathrm{SS}$ & $16 \times 16-\mathrm{SiC}$ \\
\hline \multicolumn{7}{|l|}{ Fuel Rod } \\
\hline Fuel Type & & Solid & TRISO & TRISO & TRISO & TRISO \\
\hline \multicolumn{7}{|l|}{ TRISO Kernel } \\
\hline Fissile Material & & $\mathrm{UO}_{2}$ & UN & UN & UN & UN \\
\hline $\mathrm{U}^{235}$ Enrichment & w/o & $4.5 / 4.0$ & 15.12 & 14.54 & 19.42 & 19.90 \\
\hline Fuel Density & $\mathrm{g} / \mathrm{cm}^{3}$ & 10.176 & 14.32 & 14.32 & 14.32 & 14.32 \\
\hline Kernel Diameter & $\mu \mathrm{m}$ & - & 700. & 700. & 700. & 700. \\
\hline Inner Buffer Layer Dia. & $\mu \mathrm{m}$ & - & 50. & 50. & 50. & 50. \\
\hline IPyC Layer Dia. & $\mu \mathrm{m}$ & - & 35. & 35. & 35. & 35. \\
\hline SiC Layer Dia. & $\mu \mathrm{m}$ & - & 35. & 35. & 35. & 35. \\
\hline OPyC Layer Dia. & $\mu \mathrm{m}$ & - & 20. & 20. & 20. & 20. \\
\hline \multicolumn{7}{|l|}{ Pellet } \\
\hline Matrix & & $\mathrm{UO}_{2}$ & $\mathrm{SiC}$ & $\mathrm{SiC}$ & $\mathrm{SiC}$ & $\mathrm{SiC}$ \\
\hline TRISO Packing Fraction & $\%$ & - & 55. & 55. & 55. & 55. \\
\hline Pellet Radius & $\mathrm{cm}$ & 0.4095 & 0.6725 & 0.6315 & 0.4325 & 0.3915 \\
\hline Pellet Density & $\mathrm{g} / \mathrm{cm}^{3}$ & 10.176 & 3.18 & 3.18 & 3.18 & 3.18 \\
\hline \multicolumn{7}{|l|}{ Cladding } \\
\hline Material & & $\mathrm{Zr}-4$ & SS & $\mathrm{SiC}$ & SS & $\mathrm{SiC}$ \\
\hline Density & $\mathrm{g} / \mathrm{cm}^{3}$ & 6.55 & 8.0 & 3.18 & 8.0 & 3.18 \\
\hline Inner Radius & $\mathrm{cm}$ & 0.418 & 0.681 & 0.640 & 0.441 & 0.400 \\
\hline Outer Radium & $\mathrm{cm}$ & 0.475 & 0.740 & 0.740 & 0.500 & 0.500 \\
\hline Cladding Thickness & $\mathrm{cm}$ & 0.057 & 0.059 & 0.100 & 0.059 & 0.100 \\
\hline Pellet-Cladding Gap & $\mathrm{cm}$ & 0.0085 & 0.0085 & 0.0085 & 0.0085 & 0.0085 \\
\hline \multicolumn{7}{|l|}{ Fuel Assembly } \\
\hline Array & & $16 \times 16$ & $12 \times 12$ & $12 \times 12$ & $16 \times 16$ & $16 \times 16$ \\
\hline Gap between Rods & $\mathrm{cm}$ & 0.335 & 0.235 & 0.235 & 0.285 & 0.285 \\
\hline Fuel Assembly Pitch & $\mathrm{cm}$ & 20.78 & 20.78 & 20.78 & 20.78 & 20.78 \\
\hline Fuel Rod Cell Pitch & $\mathrm{cm}$ & 1.285 & 1.175 & 1.175 & 1.285 & 1.285 \\
\hline Guide Tube Material & & $\mathrm{Zr}-4$ & SS & SS & SS & SS \\
\hline Burnable Poison & & $\mathrm{Gd}_{2} \mathrm{O}_{3}$ & $\mathrm{Er}_{2} \mathrm{O}_{3}$ & $\mathrm{Er}_{2} \mathrm{O}_{3}$ & $\mathrm{Er}_{2} \mathrm{O}_{3}$ & $\mathrm{Er}_{2} \mathrm{O}_{3}$ \\
\hline \multicolumn{7}{|l|}{ Fuel Assembly Statistics } \\
\hline Number of Fuel Rods & & 236 & 124 & 124 & 236 & 236 \\
\hline Fuel Volume $\left(\mathrm{V}_{\mathrm{f}}\right)$ & $\mathrm{cc}$ & 124.33 & 176.18 & 155.35 & 138.69 & 113.64 \\
\hline Coolant Volume $\left(V_{c}\right)$ & $\mathrm{cc}$ & 240.18 & 194.14 & 194.14 & 222.11 & 222.11 \\
\hline $\mathrm{V}_{\mathrm{c}} / \mathrm{V}_{\mathrm{f}}$ & & 1.93 & 1.10 & 1.25 & 1.115 & 1.95 \\
\hline Ratio, $V_{c} / V_{f}$ from Ref. & $\%$ & 100.0 & 57.0 & 64.7 & 82.9 & 101.2 \\
\hline Heavy Metal Loading (1/4 FA) & $\mathrm{g} / \mathrm{cm}$ & $2.79 \times 10^{2}$ & $1.19 \times 10^{2}$ & $1.05 \times 10^{2}$ & $9.40 \times 10^{1}$ & $7.70 \times 10^{1}$ \\
\hline Ratio, HM Loading from Ref. & $\%$ & 100.0 & 42.8 & 37.8 & 33.7 & 27.6 \\
\hline
\end{tabular}




\subsubsection{FCM Replacement Fuel for Westinghouse Core}

FCM replacement fuel design for Westinghouse $17 \times 17$ fuel is selected to be a $13 \times 13$ FCM UN fuel assembly of equivalent hydraulic diameter with the same initial fissile inventory. The candidate design is given in Figure 2, and a scoping analysis on core neutronic performance is carried out as in the following section.
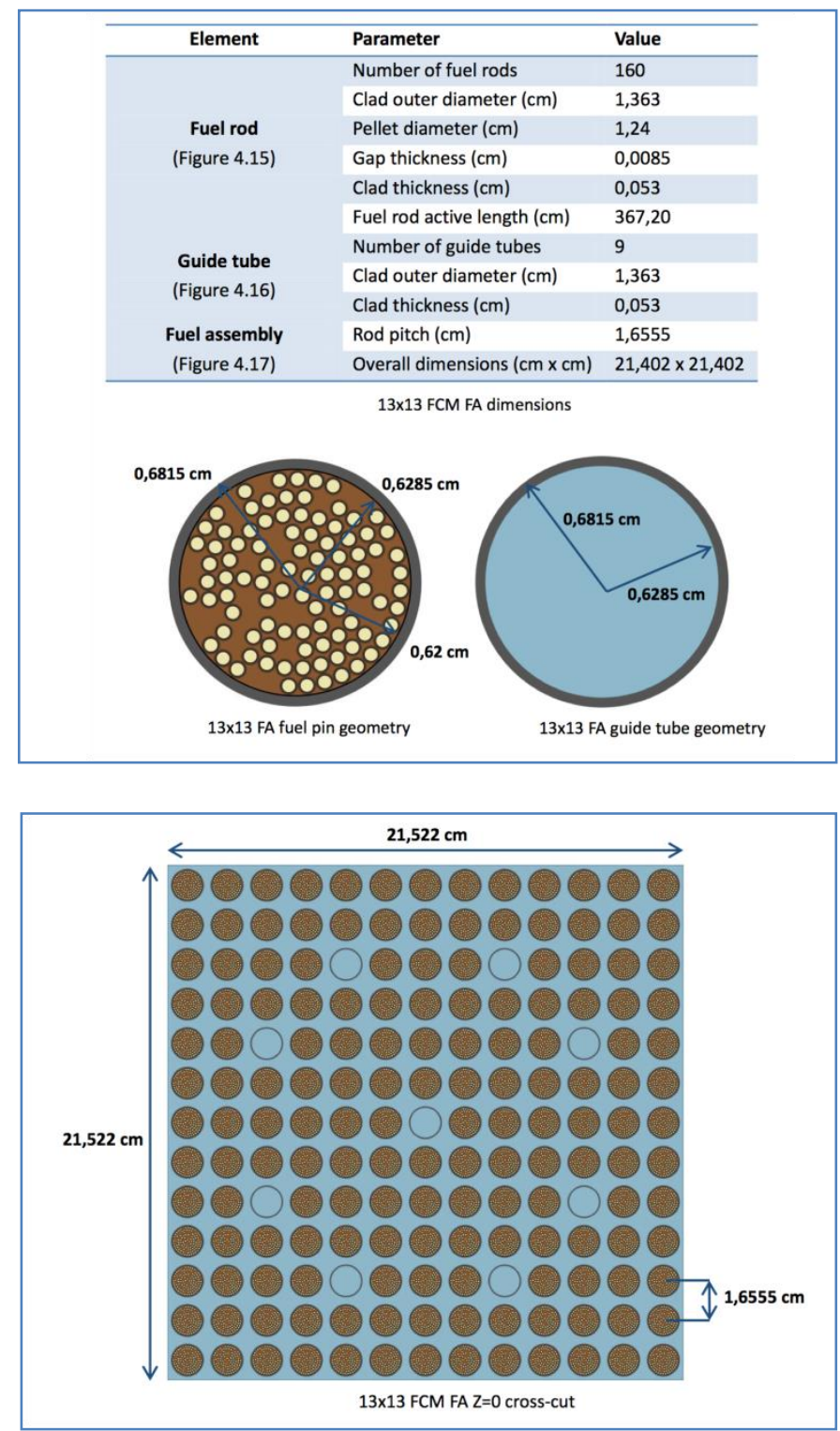

Fig. 2. Candidate 13×13 FCM UN fuel design for Westinghouse core. 


\section{NEUTRONICS EXPLORATION OF LWR FUEL ASSEMBLIES}

\subsection{TASK DESCRIPTION}

The objective of this task is to design and analyze the cycle-by-cycle reload core with FCM fuel rods in an existing LWR. The reference reactors are the OPR-1000 reactor, which is a SYSTEM 80+ type reactor, and the Westinghouse-type reactor. The goal of this year's work was to design and analyze fuel rods and assemblies fully loaded with LEU FCM fuel. In order to meet this goal, an optimization analysis was performed for the fuel rod and fuel assembly with FCM fuel for the reference LWR core condition.

\subsection{ANALYTIC METHODS AND MODELS}

\subsubsection{DeCART/MASTER}

The nuclear design and analysis of the FCM LWR core are based on the three-dimensional diffusion theory calculations for the entire core, which employ few-group cross sections generated by the twodimensional transport theory calculations performed for a unit assembly. The transport lattice calculations are performed by the DeCART code (Joo et al., 2004) to generate few-group cross sections appropriately averaged over a fuel assembly through flux volume weighting and heterogeneous form functions for the reconstruction of pin power information. The cross sections are tabularized as a function of temperature and burnup by using the PROLOG code. The nodal diffusion calculations are performed by the MASTER code (Cho et al., 2007) with the tabularized cross sections. Figure 3 shows the flowchart of the DeCART/MASTER code system for core physics analysis.

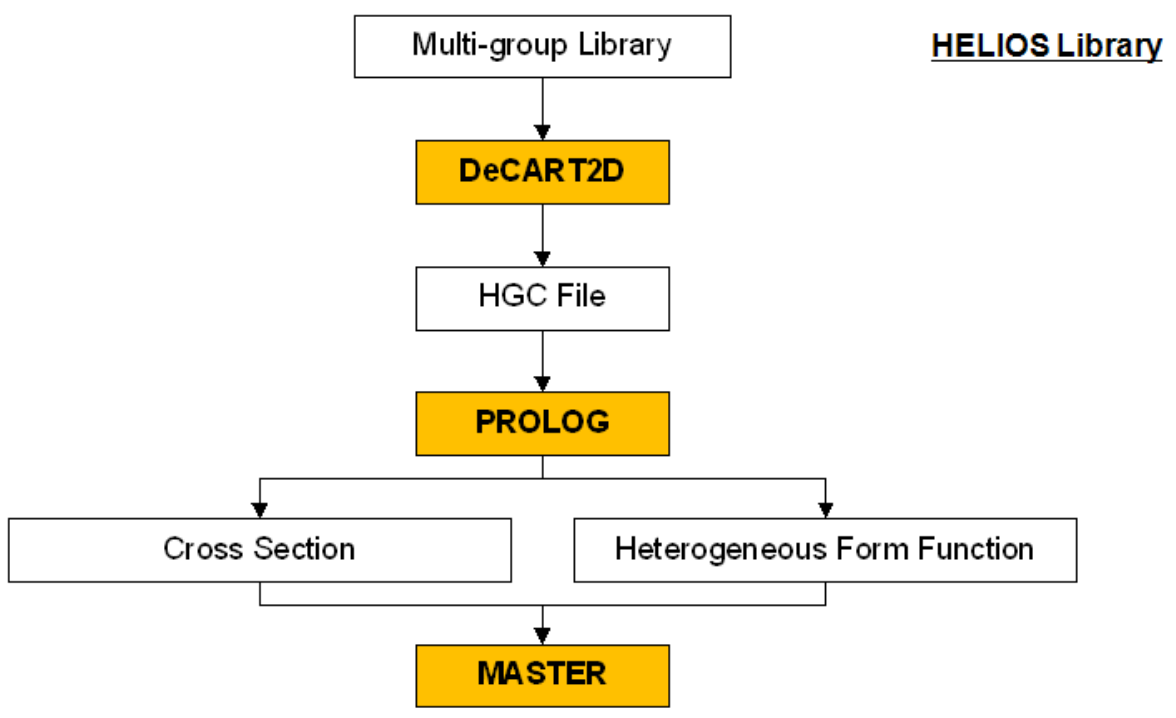

Fig. 3. DeCART/MASTER code system.

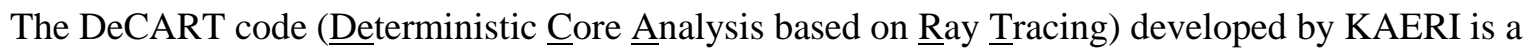
whole-core neutron transport code capable of three-dimensional (3D) core depletion analysis. This code uses the decoupling of the three-dimensional transport equation into the radial two-dimensional and the axial one-dimensional transport equations. The radial transport equations are solved by using MOC (Method of Characteristics) for planes, while the axial equations are approximated by the SP3 equations and they are solved with nodal methods. The conventional CMFD (Coarse Mesh Finite Difference) acceleration is used to enhance the rapid convergence in the eigenvalue calculation by conserving the reaction rates in a coarse mesh. DeCART treats the double heterogeneity of the particle fuels by using the 
Sanchez method, and it uses the subgroup method for resonance self-shielding treatment. Criticality spectra can be obtained through the B1 method by homogenizing the whole domain. Local scalar fluxes are modified by the criticality spectra to consider the neutron leakage effect. The criticality spectra are used in obtaining few-group constants and 1-group cross sections to be used in the burnup calculations. Burnup calculations are performed by the exponential matrix method for which a solution is obtained by the Krylov subspace method. DeCART generates the HGC (Homogenized Group Constants) file, which will be used to edit few-group constants, power distributions, and various data required for multidimensional diffusion core calculation. To model and analyze the two-dimensional fuel assembly, a zdirectional $1 \mathrm{~cm}$ length is assumed, and reflective conditions are imposed on the top and bottom boundaries.

MASTER (Multi-purpose Analyzer for Static and Transient Effects of Reactor) developed by KAERI is a nuclear design code based on the two-group diffusion theory to calculate the steady-state and transient PWR core in a three-dimensional Cartesian or hexagonal geometry. Its neutronics model solves the spacetime-dependent neutron diffusion equations with SENM (Source Expansion Nodal Method) for Cartesian geometries and with TPEN (Triangle-based Polynomial Expansion Nodal) method for hexagonal geometries. The transverse leakage model that solves the transverse averaged one-dimensional diffusion equation is treated by a parabolic approximation. The conventional CMFD is implemented to accelerate the convergence of iteration process. MASTER performs microscopic depletion calculations using microscopic cross sections provided by DeCART and also has reconstruction capabilities of pin power information by the use of MSS-IAS (Method of Successive Smoothing with Improved Analytic Solution). For the thermal-hydraulic calculation, fuel temperature table or COBRA3-C/P can be used, selectively.

\subsubsection{McCARD}

The continuous energy Monte Carlo code McCARD (Shim et al., 2012) is used for the neutronic analysis of FCM fuel rod and fuel assembly. McCARD was developed at Seoul National University, Korea. It has a built-in depletion routine; thus, it can be used in a stand-alone mode for the reactor depletion analysis. In a depletion calculation, it is important to consider fission products as accurately and extensively as possible. In the McCARD depletion calculations, all actinides and over 160 fission product nuclides are considered and the fission product poisoning can be accounted with high accuracy. The cross section libraries are generated from the ENDF-B/VI and B/VII data.

McCARD can also directly handle the doubly-heterogeneous fuel (TRISO particles in SiC matrix rods in fuel bundles) in LWRs. In particular, the randomness of the TRISO fuel particles can also be taken into consideration, as the location of the TRISO fuel particles is randomly determined. The McCARD code can be run optimally on parallel nodes of a cluster.

\subsubsection{SERPENT}

SERPENT is a three-dimensional continuous-energy Monte Carlo reactor physics and burnup calculation code, whose development started at the VTT Technical Research Centre of Finland in 2004 under the working title "Probabilistic Scattering Game" (PSG). The SERPENT code is written in standard ANSI-C language and reads continuous-energy interaction data from ACE format cross section libraries, such as the JEFF-3.1.1.xsdata cross section library.

SERPENT uses a model based on universes, where geometry is divided into separate independent levels nested one inside the other. Materials can be modeled by defining density, nuclide fraction composition, and temperature. The JEFF-3.1.1.xsdata library provides cross sections at fixed nuclide temperatures $(300 \mathrm{~K}, 600 \mathrm{~K}, 900 \mathrm{~K}, 1200 \mathrm{~K}, 1500 \mathrm{~K}$ and $1800 \mathrm{~K})$. Cross sections at intermediate temperatures are obtained by interpolating the tabulated data. 
SERPENT is also used for burnup calculation. The code uses an internal calculation routine for solving the Bateman equations, which describe abundances and activities in a decay chain as a function of time, based on decay rates and initial abundances. The burnup calculation allows changes in the material compositions and radioactive decay.

\subsection{FCM FUEL ASSEMBLY DESIGN FOR OPR-1000 CORE}

The simplest approach to using FCM fuel in a LWR is to use FCM compacts that have the same diameter as the solid pellets in the same cladding tube as in the reference reactors. If these FCM compacts are used in LWR, the heavy metal inventory of a fuel assembly will be much lower than that of the reference solid oxide fuel assembly. In order to increase the heavy metal inventory in the FCM fuel assembly, the following measures were taken: (1) introduce the $12 \times 12$ fuel assembly grid geometry, (2) introduce the larger fuel kernel diameter TRISO, (3) adopt the higher density fuel UN (14.32 g/ $\left.\mathrm{cm}^{3}\right)$ compared with the $\mathrm{UO}_{2}$ fuel $\left(10.176 \mathrm{~g} / \mathrm{cm}^{3}\right)$. Also, the SS304 and SiC cladding materials in an FCM fuel assembly were selected to replace the zirconium-alloy cladding in the fuel assembly.

\subsubsection{Reference Data and Geometries}

Table 5 shows the general core data of the OPR-1000 reactor. The OPR-1000 is a 2815 MWth reactor, operating with 177 fuel assemblies in the core. The height of the active core is $381 \mathrm{~cm}$. Table 6 shows the fuel assembly pitch, cell pitch, fuel rod, guide tube, and control rod dimensions of the reference fuel assembly (Kim, 2010). Figure 4 shows the fuel pin cell and 16×16 fuel assembly configurations. Figure 5 shows the FCM fuel pin cell and $12 \times 12$ fuel assembly configurations. It is important to note that the location and dimensions of control rods and guide tubes are the same in both configurations. The FCM fuel rod dimensions vary with the selected cladding material and fuel assembly configuration and will be described in the next section. The TRISO particle dimensions used in this work are shown in Table 7, with Figure 6 showing the layout of the particle. A $50 \mu \mathrm{m}$ buffer thickness is used, compared with the $100 \mu \mathrm{m}$ buffer thickness of the typical HTR TRISO. The smaller thickness of the buffer reflects the lower operating temperature and the fact that UN fuel is used. In the high-temperature reactor (HTR), application, $\mathrm{UCO}$ or $\mathrm{UO}_{2}$ is used as fuel, and $\mathrm{CO}$ gas is released with burnup, contributing to the internal pressure in the particle. However, because $\mathrm{CO}$ gas is not released in the UN fuel, the buffer thickness of the UN TRISO used in the FCM fuel can be decreased.

Table 5. General core data

\begin{tabular}{|l|c|c|}
\hline \multicolumn{1}{|c|}{ Item } & Unit & Value \\
\hline Thermal power & $\mathrm{MW}$ & 2815 \\
\hline Number of fuel assemblies in core & & 177 \\
\hline Active core height & $\mathrm{cm}$ & 381 \\
\hline Inlet temperature & ${ }^{\circ} \mathrm{C}$ & 295.8 \\
\hline Core average moderator temperature & ${ }^{\circ} \mathrm{C}$ & 312.2 \\
\hline Average cladding temperature & ${ }^{\circ} \mathrm{C}$ & 338.9 \\
\hline Effective fuel temperature & ${ }^{\circ} \mathrm{C}$ & 687.8 \\
\hline Reference soluble boron concentration & $\mathrm{ppm}$ & 500 \\
\hline Coolant pressure & $\mathrm{bar}$ & 155.13 \\
\hline
\end{tabular}


Table 6. Reference fuel assembly data

\begin{tabular}{|c|c|c|c|}
\hline & Item & Unit & Value \\
\hline Assembly pit & & $\mathrm{cm}$ & 20.78 \\
\hline Cell pitch & & $\mathrm{cm}$ & 1.285 \\
\hline & Pellet radius & $\mathrm{cm}$ & 0.4095 \\
\hline Fuel & Clad inner radius & $\mathrm{cm}$ & 0.4180 \\
\hline & Clad outer radius & $\mathrm{cm}$ & 0.4750 \\
\hline & Inner radius & $\mathrm{cm}$ & 1.1450 \\
\hline Guide tube & Outer radius & $\mathrm{cm}$ & 1.2450 \\
\hline & Control pellet radius & $\mathrm{cm}$ & 0.9360 \\
\hline Control rod & Clad inner radius & $\mathrm{cm}$ & 0.9475 \\
\hline & Clad outer radius & $\mathrm{cm}$ & 1.0365 \\
\hline
\end{tabular}
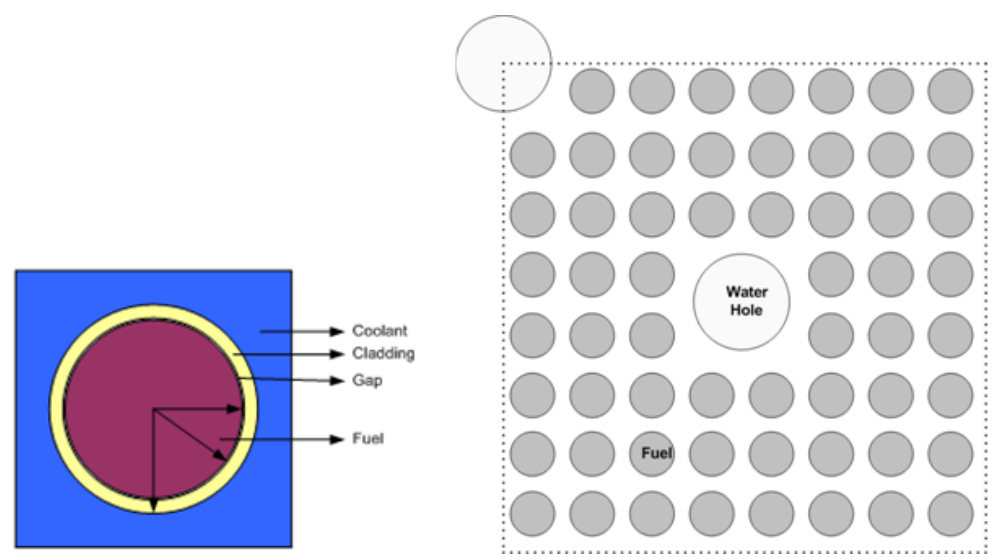

Fig. 4. Configurations of the fuel pin cell and $16 \times 16$ quarter fuel assembly.

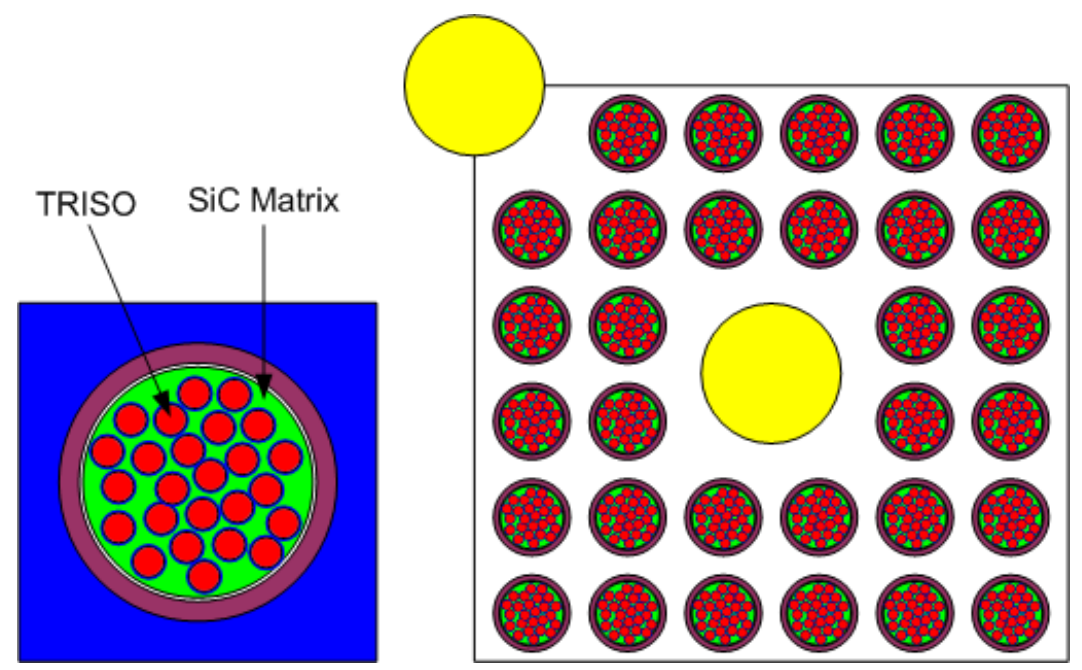

Fig. 5. Configuration of the FCM fuel pin cell and $12 \times 12$ quarter fuel assembly. 
Table 7. TRISO particle dimensions used in FCM fuel

\begin{tabular}{|c|c|c|}
\hline Layers & Parameter & LWR-FCM \\
\hline \multirow{3}{*}{ Kernel } & Material & Variable \\
\cline { 2 - 3 } & Diameter & Variable \\
\cline { 2 - 3 } & Density & $14.32 \mathrm{~g} / \mathrm{cm}^{3}$ \\
\hline \multirow{2}{*}{ Buffer layer } & Thickness & $50 \mu \mathrm{m}$ \\
\cline { 2 - 3 } & Density & $1.05 \mathrm{~g} / \mathrm{cm}^{3}$ \\
\hline \multirow{2}{*}{ Inner PyC coating } & Thickness & $35 \mu \mathrm{m}$ \\
\cline { 2 - 3 } & Density & $1.9 \mathrm{~g} / \mathrm{cm}^{3}$ \\
\hline \multirow{2}{*}{ SiC coating } & Thickness & $35 \mu \mathrm{m}$ \\
\cline { 2 - 3 } & Density & $3.18 \mathrm{~g} / \mathrm{cm}^{3}$ \\
\hline \multirow{2}{*}{ Outer PyC coating } & Thickness & $20 \mu \mathrm{m}$ \\
\cline { 2 - 3 } & Density & $1.9 \mathrm{~g} / \mathrm{cm}^{3}$ \\
\hline
\end{tabular}

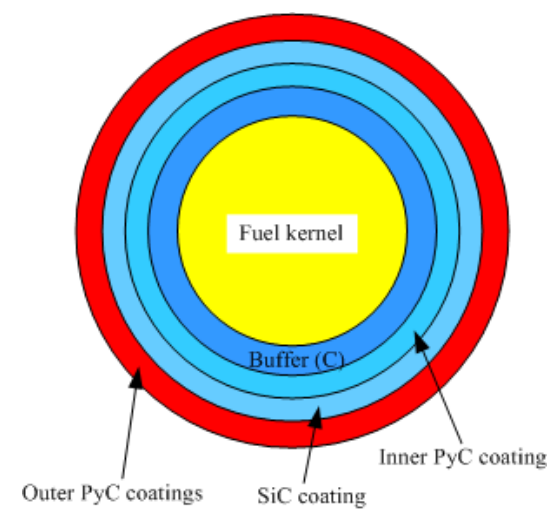

Fig. 6. Layout of the TRISO particle.

\subsubsection{Scoping Analysis for the Fuel Assembly Design Selection}

As mentioned above, $12 \times 12$ and $16 \times 16$ FCM fuel assembly configurations are considered in this work. This work also considered two types of cladding materials: SS304 and SiC materials. For the optimized fuel rod design, this section describes the sensitivity of the kernel diameters and the packing fractions of the TRISO in an FCM fuel rod. The maximum packing fraction considered for the TRISO fuel is 55\%. This packing fraction is above the referenced maximum for Nano-Infiltration and Transient Eutecticphase (NITE)-sintered SIC compacts (45\%), but feasible with the lower temperature and pressure processes indicated by Ceramatec and USNC. From the results of the previous study (C.K. Jo, 2012), the use of $\mathrm{UO}_{2}$ and UCO fuels in an FCM fuel concept will result in a sharply lower k-infinity because of the smaller loading of the heavy metal inventory. The enrichment of the ${ }^{235} \mathrm{U}$ in the $\mathrm{UN}$ fuel is selected so as to produce the same k-infinity behavior in the FCM fuel assembly as in the solid $\mathrm{UO}_{2}$ fuel assembly of the OPR-1000.

Table 8 shows the change in heavy metal inventory with kernel diameter in the quarter fuel assembly for the four cases: $(1,2) 12 \times 12$ fuel assembly with SS304 cladding and SiC cladding and $(3,4) 16 \times 16$ fuel assembly with SS304 cladding and SiC cladding, respectively. From the results of the mechanical 
analysis, the thickness of the SS304 cladding is set to $0.059 \mathrm{~cm}$ and the $0.1 \mathrm{~cm}$ cladding thickness for SiC cladding is used, based on manufacturing consideration. The 55\% TRISO packing fraction is used for the kernel diameter sensitivity study. The k-infinity changes of the fuel assembly for four cases with kernel diameter are shown in Figure 7. From the Table 8, the heavy metal inventory of the fuel assembly is very affected by kernel diameter. In order to increase the heavy metal inventory, the kernel diameter of TRISO should be larger. In this work, the maximum kernel diameter is set to $700 \mu \mathrm{m}$ considering the manufacture.

Table 9 shows the heavy metal inventory as a function of TRISO packing fraction in the quarter fuel assembly for the four cases. The $700 \mu \mathrm{m}$ kernel diameter in the TRISO particle is kept constant. The k-infinity changes of the fuel assembly for four cases with TRISO packing fraction are shown in Figure 8. As shown in Table 9, the heavy metal inventory of the fuel assembly is strongly affected by TRISO packing fraction. In order to increase the heavy metal inventory, the TRISO packing fraction should be larger. Alternatively, the fissile inventory can be increased by increasing the enrichment.

From the results of the sensitivity analysis, the selected design specifications of the fuel rods and assemblies for the $12 \times 12$ and $16 \times 16$ fuel assemblies are shown in Table 10 . The assembly pitch is $20.78 \mathrm{~cm}$ for all cases, and the cell pitches are $1.715 \mathrm{~cm}$ and $1.285 \mathrm{~cm}$ for the $12 \times 12$ and $16 \times 16$ fuel assemblies, respectively. The pellet radius is determined by the cladding material and the fuel assembly grid $(12 \times 12$ or $16 \times 16)$. The pellet radius is smaller for the SiC clad case than for the SS304 clad in the same fuel assembly grid because of the larger $\mathrm{SiC}$ clad thickness required. The uranium enrichment for the FCM fuel assembly cases is determined by the criterion of having the same k-infinity value at 1100 EFPD (Effective Full Power Day), as mentioned above. Only UN fuel kernels were considered for the FCM fuel assemblies. From the results of the previous study (Jo et al., 2012), the use of $\mathrm{UO}_{2}$ and UCO fuels in an FCM fuel concept would sharply reduce the k-infinity value because of the smaller loading of heavy metal. The material of the guide tube in an FCM fuel assembly is changed from Zircaloy-4 to SS304 in order to completely remove Zircaloy-4 material from the core.

Table 8. Comparison of the heavy metal inventory with kernel diameter

\begin{tabular}{|c|c|c|c|c|c|}
\hline & & $K D=400 \mu \mathrm{m}$ & $K D=500 \mu m$ & $K D=600 \mu m$ & $K D=700 \mu \mathrm{m}$ \\
\hline \multirow{2}{*}{$\begin{array}{c}12 \times 12 \mathrm{FA},{ }^{a} \\
\text { Clad SS304, } \\
{ }^{235} \mathrm{U} 15.12 \mathrm{w} / \mathrm{o}\end{array}$} & $\mathrm{HM}(\mathrm{g}) / \mathrm{cm} /(1 / 4 \mathrm{FA})$ & $6.67 \mathrm{E}+01$ & $8.63 \mathrm{E}+01$ & $1.04 \mathrm{E}+02$ & $1.19 \mathrm{E}+02$ \\
\hline & FCM/Solid $\mathrm{UO}_{2}$ & $23.92 \%$ & $30.95 \%$ & $37.24 \%$ & $42.82 \%$ \\
\hline \multirow{2}{*}{$\begin{array}{l}12 \times 12 \mathrm{FA}, \\
\text { Clad SiC, } \\
{ }^{235} \mathrm{U} 14.54 \mathrm{w} / \mathrm{o}\end{array}$} & $\mathrm{HM}(\mathrm{g}) / \mathrm{cm} /(1 / 4 \mathrm{FA})$ & $5.88 \mathrm{E}+01$ & $7.61 \mathrm{E}+01$ & $9.16 \mathrm{E}+01$ & $1.05 \mathrm{E}+02$ \\
\hline & FCM/Solid $\mathrm{UO}_{2}$ & $21.09 \%$ & $27.29 \%$ & $32.84 \%$ & $37.76 \%$ \\
\hline \multirow{2}{*}{$\begin{array}{c}16 \times 16 \mathrm{FA}, \\
\text { Clad SS304, } \\
{ }^{235} \mathrm{U} 19.42 \mathrm{w} / \mathrm{o}\end{array}$} & $\mathrm{HM}(\mathrm{g}) / \mathrm{cm} /(1 / 4 \mathrm{FA})$ & $5.25 \mathrm{E}+01$ & $6.79 \mathrm{E}+01$ & $8.17 \mathrm{E}+01$ & $9.40 \mathrm{E}+01$ \\
\hline & FCM/Solid $\mathrm{UO}_{2}$ & $18.83 \%$ & $24.36 \%$ & $29.32 \%$ & $33.71 \%$ \\
\hline \multirow{2}{*}{$\begin{array}{c}\text { 16×16FA, } \\
\text { Clad SiC, } \\
{ }^{235} \mathrm{U} 19.90 \text { wo }\end{array}$} & $\mathrm{HM}(\mathrm{g}) / \mathrm{cm} /(1 / 4 \mathrm{FA})$ & $4.30 \mathrm{E}+01$ & $5.57 \mathrm{E}+01$ & $6.70 \mathrm{E}+01$ & $7.70 \mathrm{E}+01$ \\
\hline & FCM/Solid $\mathrm{UO}_{2}$ & $15.43 \%$ & $19.96 \%$ & $24.02 \%$ & $27.62 \%$ \\
\hline \multicolumn{2}{|c|}{ Solid $\mathrm{UO}_{2}$ (1/4 FA Mass, g/cm) } & $2.79 \mathrm{E}+02$ & & & \\
\hline
\end{tabular}

${ }^{a} \mathrm{FA}=$ fuel assembly 


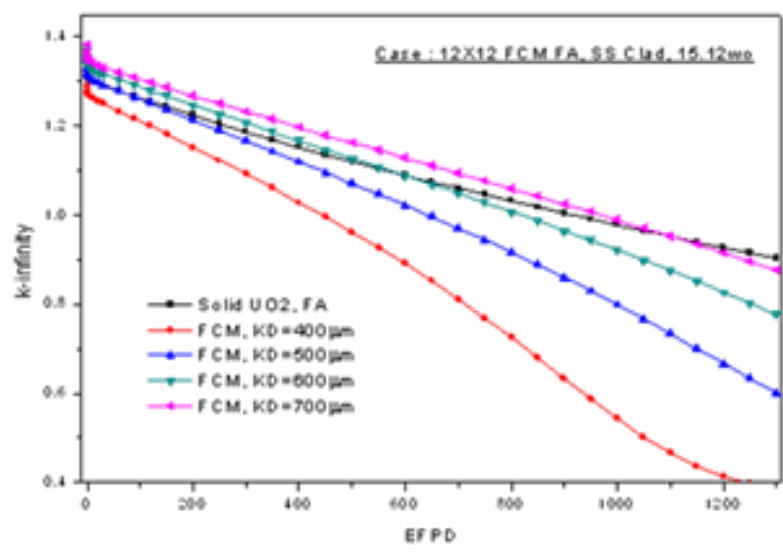

(a) $12 \times 12$ FA. SS Clad Case

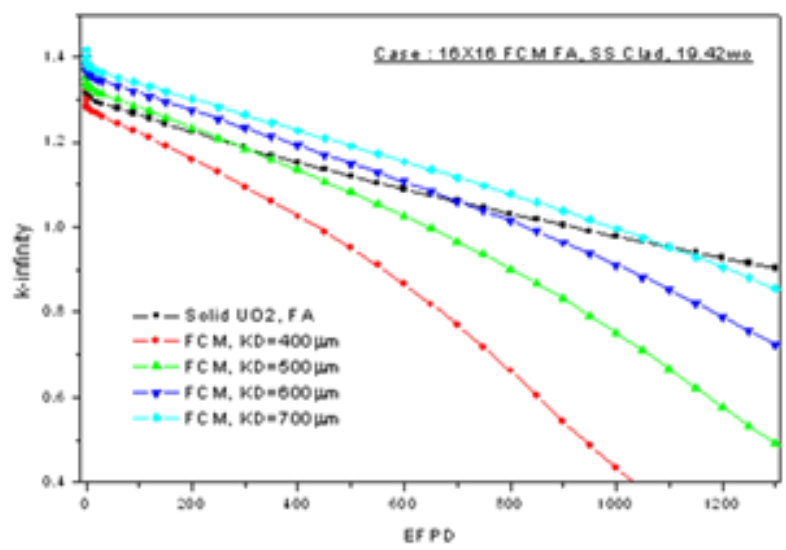

(c) $16 \times 16$ FA. SS Clad Case

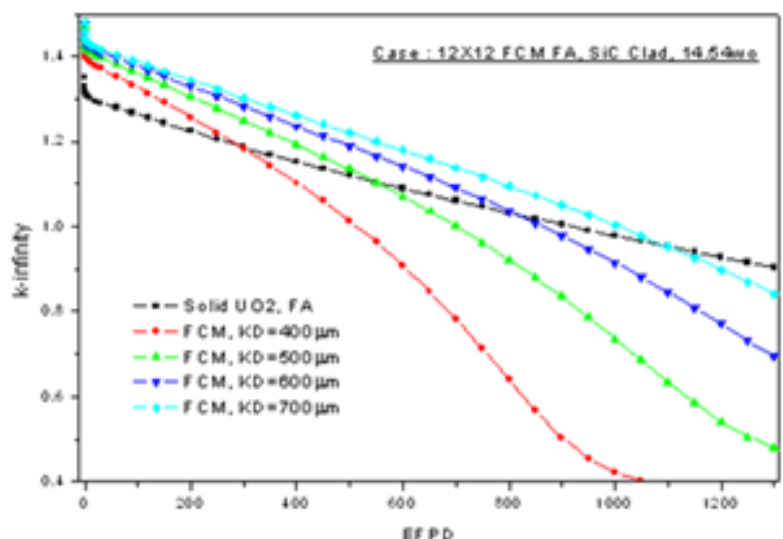

(b) 12X12FA. SiC Clad Case

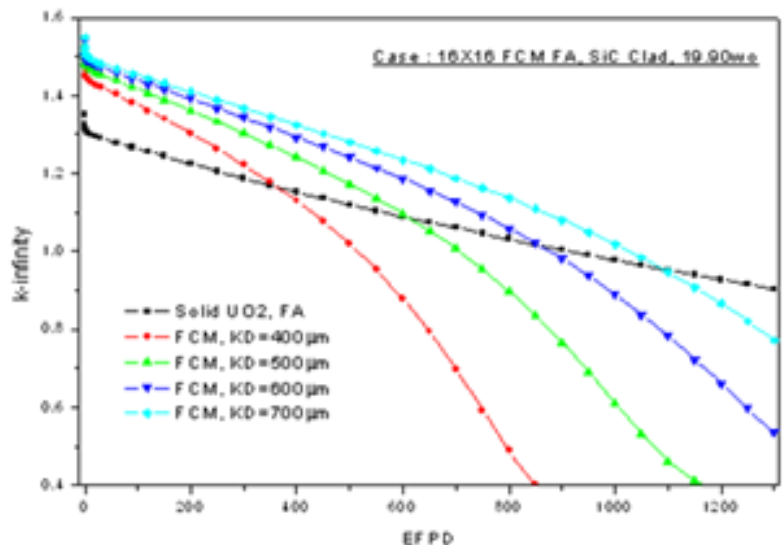

(d) 16X16 FA. SiC Clad Case

Fig. 7. k-infinity changes with kernel diameter and cladding materials.

Table 9. Comparison of the heavy metal inventory with TRISO packing fraction

\begin{tabular}{|c|c|c|c|c|c|}
\hline & & $\mathrm{PF}=30 \%$ & $\mathrm{PF}=40 \%$ & $\mathrm{PF}=45 \%$ & $\mathrm{PF}=55 \%$ \\
\hline \multirow{2}{*}{$\begin{array}{l}12 \times 12 \mathrm{FA}, \\
\text { Clad SS304, } \\
{ }^{235} \mathrm{U} 15.12 \mathrm{w} / \mathrm{o}\end{array}$} & $\mathrm{HM}(\mathrm{g}) / \mathrm{cm} /(1 / 4 \mathrm{FA})$ & $6.51 \mathrm{E}+01$ & $8.68 \mathrm{E}+01$ & $9.77 \mathrm{E}+01$ & $1.19 \mathrm{E}+02$ \\
\hline & FCM/Solid $\mathrm{UO}_{2}$ & $23.36 \%$ & $31.14 \%$ & $35.03 \%$ & $42.82 \%$ \\
\hline \multirow{2}{*}{$\begin{array}{l}12 \times 12 \mathrm{FA}, \\
\text { Clad SiC, } \\
{ }^{235} \mathrm{U} 14.54 \mathrm{w} / \mathrm{o}\end{array}$} & $\mathrm{HM}(\mathrm{g}) / \mathrm{cm} /(1 / 4 \mathrm{FA})$ & $5.74 \mathrm{E}+01$ & $7.66 \mathrm{E}+01$ & $8.61 \mathrm{E}+01$ & $1.05 \mathrm{E}+02$ \\
\hline & FCM/Solid $\mathrm{UO}_{2}$ & $20.59 \%$ & $27.46 \%$ & $30.89 \%$ & $37.76 \%$ \\
\hline \multirow{2}{*}{$\begin{array}{l}16 \times 16 \text { FA, } \\
\text { Clad SS304, } \\
{ }^{235} \text { U 19.42w/o }\end{array}$} & $\mathrm{HM}(\mathrm{g}) / \mathrm{cm} /(1 / 4 \mathrm{FA})$ & $5.13 \mathrm{E}+01$ & $6.83 \mathrm{E}+01$ & $7.69 \mathrm{E}+01$ & $9.40 \mathrm{E}+01$ \\
\hline & FCM/Solid $\mathrm{UO}_{2}$ & $18.38 \%$ & $24.51 \%$ & $27.58 \%$ & $33.71 \%$ \\
\hline \multirow{2}{*}{$\begin{array}{l}16 \times 16 \mathrm{FA}, \\
\text { Clad SiC, } \\
{ }^{235} \mathrm{U} 19.90 \mathrm{wo}\end{array}$} & $\mathrm{HM}(\mathrm{g}) / \mathrm{cm} /(1 / 4 \mathrm{FA})$ & $4.20 \mathrm{E}+01$ & $5.60 \mathrm{E}+01$ & $6.30 \mathrm{E}+01$ & $7.70 \mathrm{E}+01$ \\
\hline & FCM/Solid $\mathrm{UO}_{2}$ & $15.06 \%$ & $20.09 \%$ & $22.60 \%$ & $27.62 \%$ \\
\hline \multicolumn{2}{|c|}{ Solid $\mathrm{UO}_{2}(1 / 4$ FA Mass, $\mathrm{g} / \mathrm{cm})$} & $2.79 \mathrm{E}+02$ & & & \\
\hline
\end{tabular}




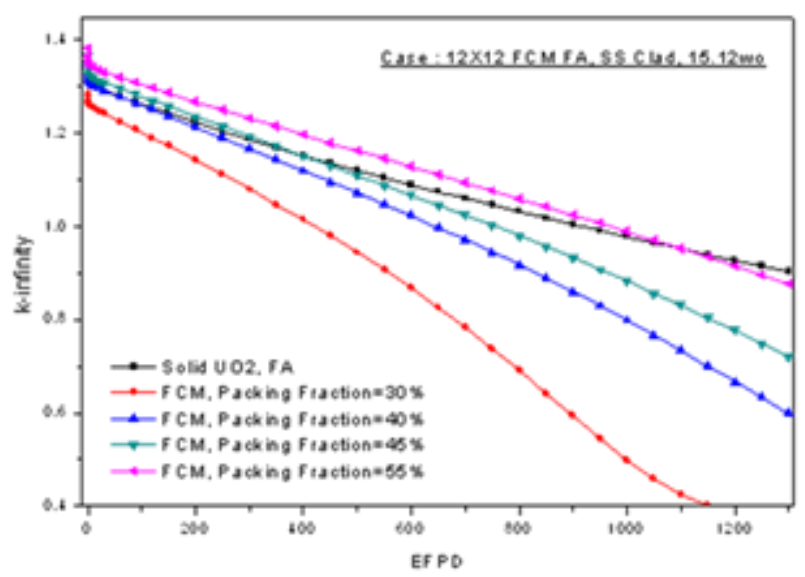

(a) 12X12FA. SS Clad Case

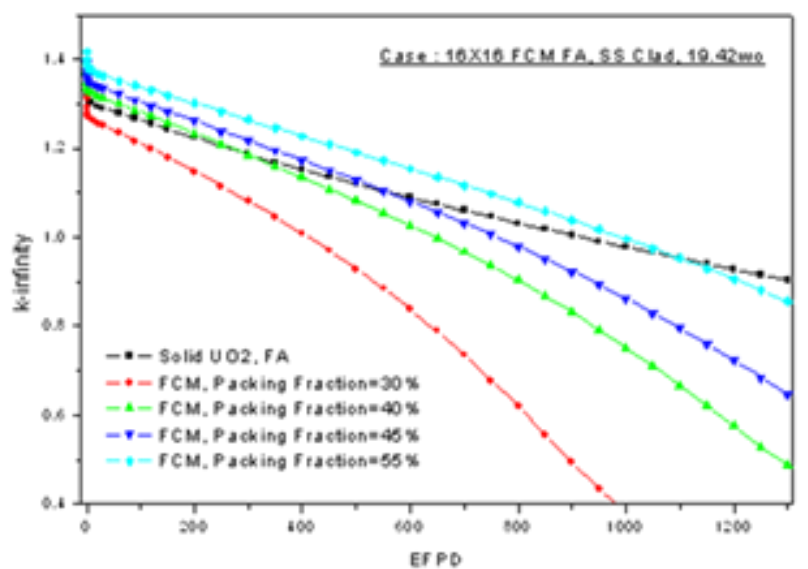

(c) $16 \times 16$ FA. SS Clad Case

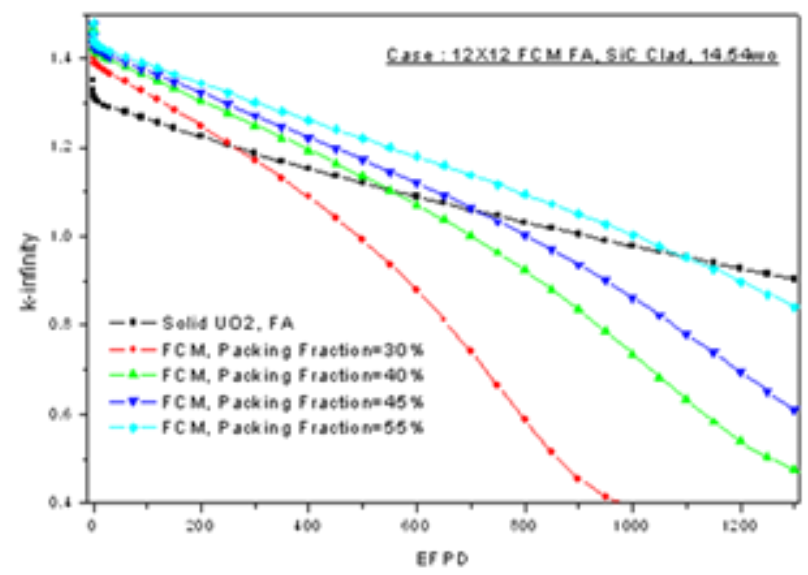

(b) $12 \times 12$ FA. SiC Clad Case

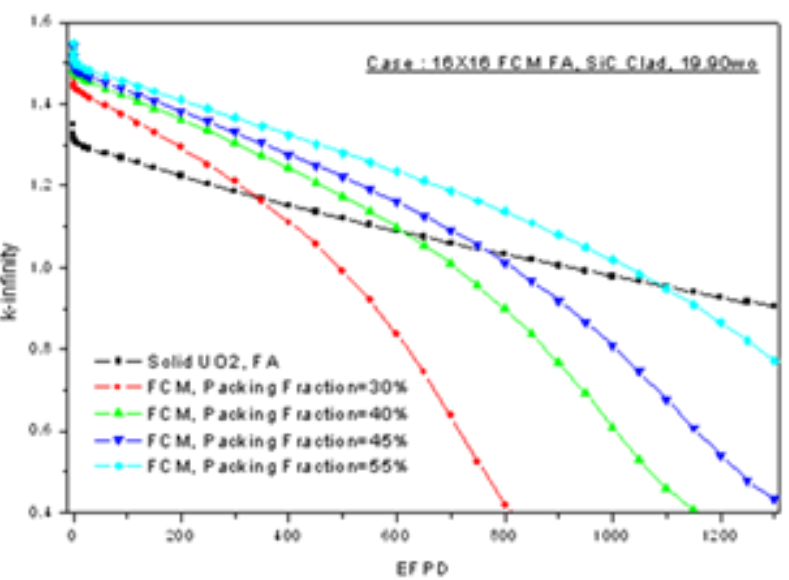

(d) 16X16 FA. SiC Clad Case

Fig. 8. k-infinity changes with TRISO packing fraction and cladding materials. 
Table 10. Specifications of fuel rods and fuel assemblies for LWR FCM

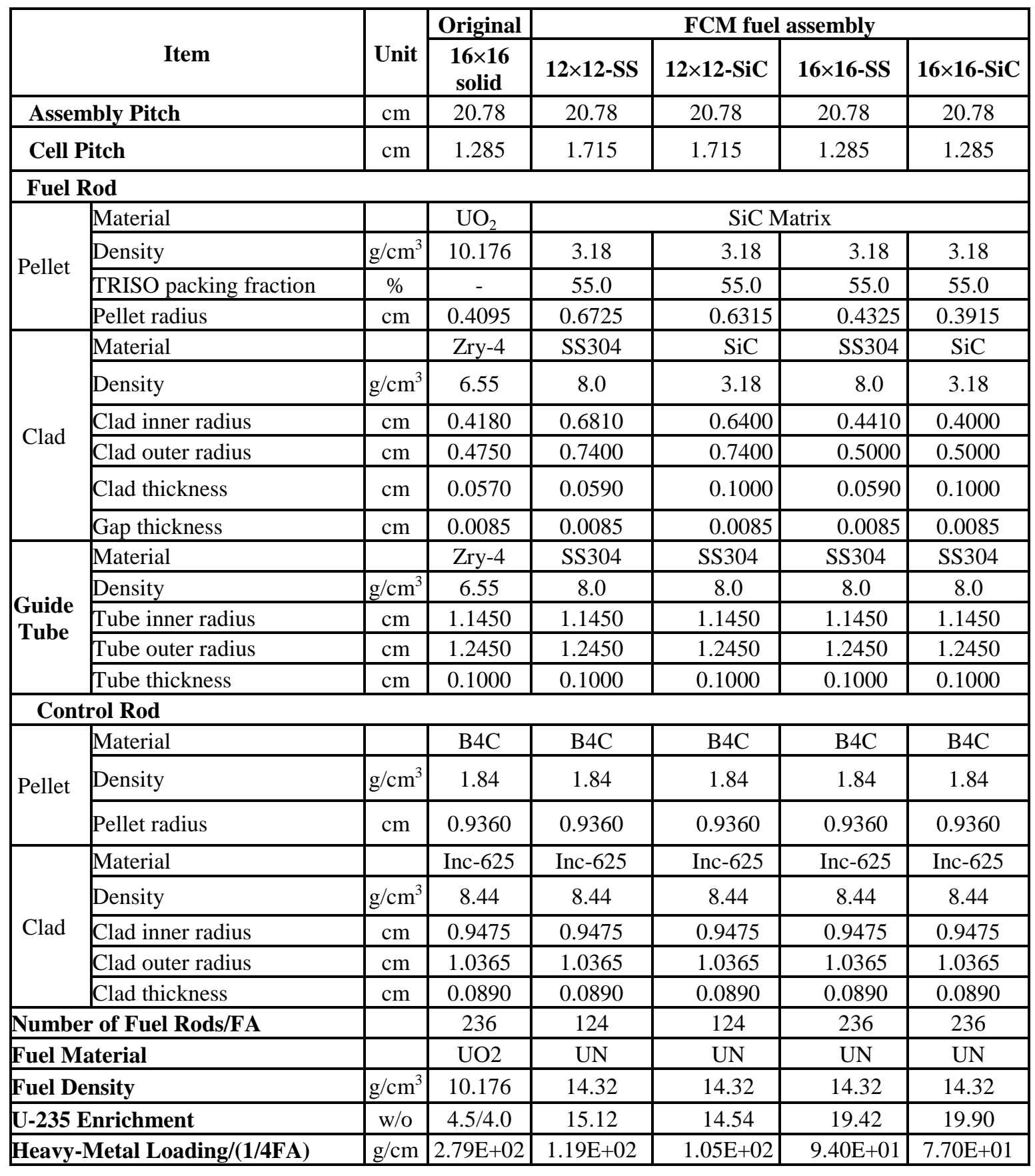

\subsubsection{Results of the Fuel Assembly Analysis}

This section describes the results of the fuel assembly analysis, using the DeCART2D code. The temperature of the TRISO fuel and $\mathrm{SiC}$ matrix is set to $687.8^{\circ} \mathrm{C}$, the temperature of the cladding is set to $338.9^{\circ} \mathrm{C}$, and the temperature of the coolant and guide tube is set to $312.20^{\circ} \mathrm{C}$. These values are taken from the reference OPR-1000 data. A 500 ppm soluble boron concentration in the coolant is considered. The assembly-wise power generation is assumed identical for all cases. 
Figure 9 shows the reduction in neutron multiplication factor (k-infinity) of the FCM fuel assembly with depletion, in comparison with the reference $16 \times 16$ solid oxide fuel assembly. From the figure, the multiplication factor of the FCM fuel assembly decreases more rapidly with depletion. As determined by selection criteria, the multiplication factors of all cases are nearly identical at 1100 EFPD. This was obtained by adjusting the U-235 enrichment. In this work, a 55\% value for the TRISO packing fraction was used in all FCM cases.

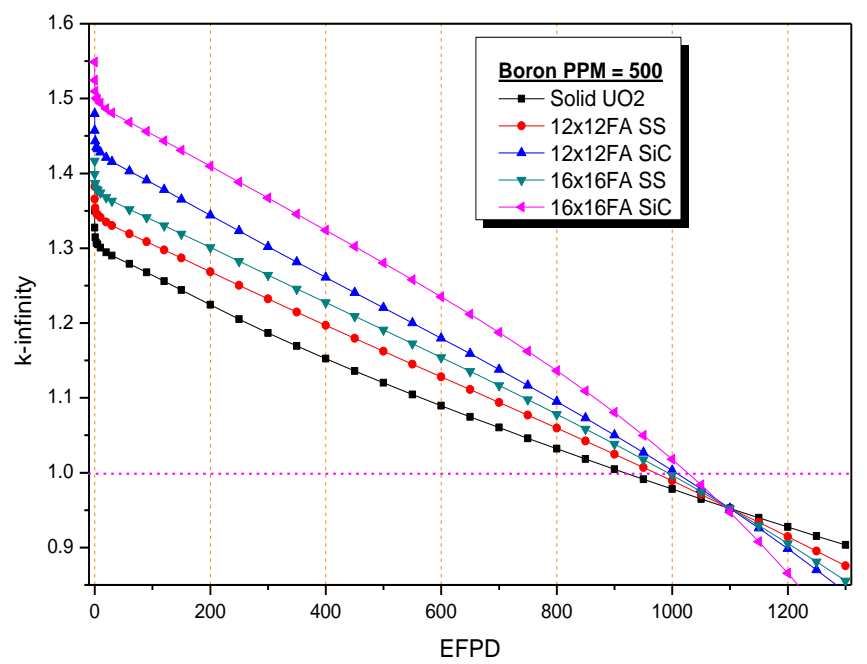

Fig. 9. Neutron multiplication factors of FCM fuel assembly.

A consistently negative reactivity feedback is essential to the safe operation of a nuclear reactor.

Figure 10 shows the moderator temperature coefficients (MTC) of the FCM fuel assemblies compared to the reference solid $\mathrm{UO}_{2}$ fuel assembly. Figure 10 indicates that the MTC of the FCM fuel assembly is less negative than that of the solid $\mathrm{UO}_{2}$ fuel assembly. In particular, the MTC of the $16 \times 16 \mathrm{FCM}$ fuel assembly with $\mathrm{SiC}$ cladding has positive values at the higher burnup region. This is caused by the smaller heavy metal inventory, as seen in Table 10 and by the consequent excessive softening in the neutron spectrum with fuel depletion as shown in Figure 10. From Figure 11, it can be seen that the thermal neutron spectrum of the solid $\mathrm{UO}_{2}$ fuel does not change significantly during the depletion, but the neutron spectrum of the FCM fuel becomes significantly more thermal during depletion. This affects the MTC. Also, the MTC is affected by the soluble boron concentration in the coolant, as shown in Figure 12. The MTC of fuel assembly at the higher soluble boron concentration is less negative than that of the medium soluble boron concentration case. The MTC for nearly zero soluble boron concentration is more negative. The behavior of the MTC at higher burnups is a mostly a consequence of the excessive depletion in the FCM fuel, driven by the nature of the assembly level calculations. It is reasonable to expect that the behavior of the MTC will show improvement in the course of the full-core analysis.

Figure 13 shows the fuel temperature coefficient (FTC) of the solid $\mathrm{UO}_{2}$ fuel assembly and the UN FCM fuel assemblies. From the figure, the fuel temperature coefficient is very benign and not significantly affected by the cladding materials and the fuel assembly grids. The FTC is also not affected by the soluble boron concentration in the coolant. 
Figure 14 shows the boron reactivity worth of the solid $\mathrm{UO}_{2}$ fuel assembly and the UN FCM fuel assemblies. From the figure, the boron reactivity worth of the UN FCM fuel assembly with SS304 cladding material is not significantly affected by the fuel assembly grid. However, the boron reactivity worth of the UN FCM fuel assemblies with SiC cladding material is more greatly affected at the higher burnup region, again due to the greater thermalization of the spectrum. Also, the boron reactivity worth is not significantly affected by the soluble boron concentration in the coolant.

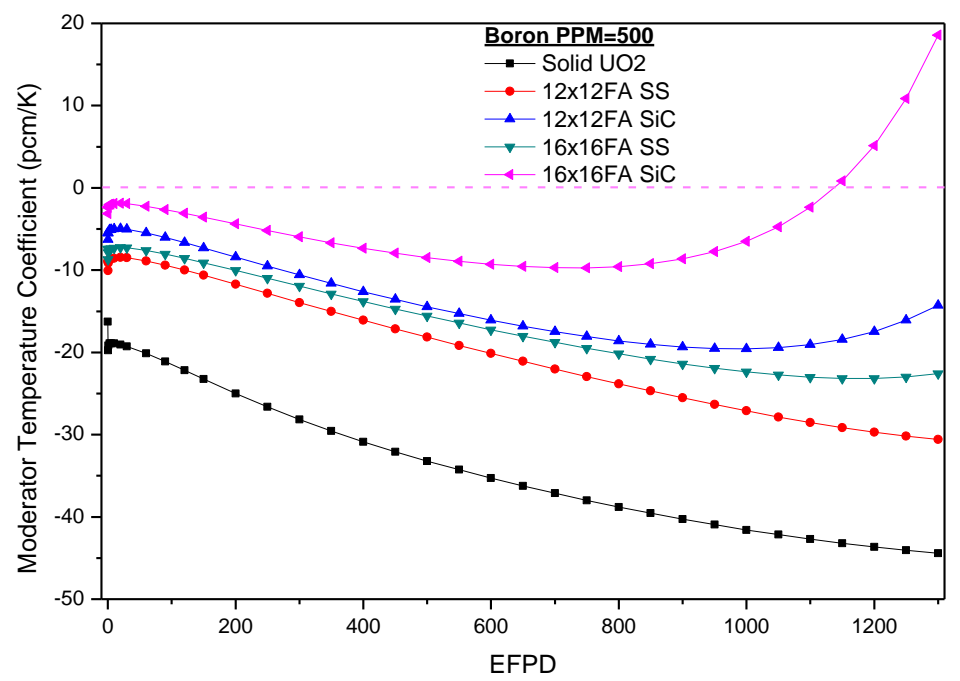

Fig. 10. Moderator temperature coefficients (soluble boron concentration $=500 \mathrm{ppm}$ ).
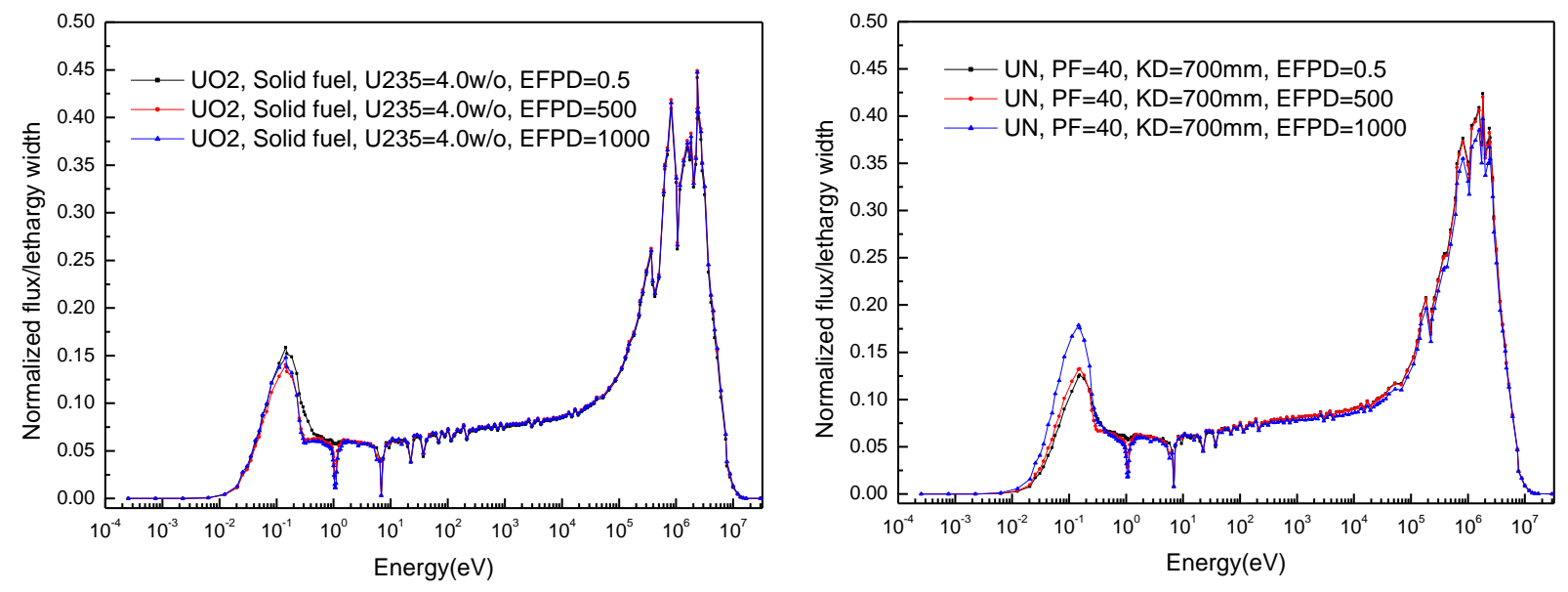

Fig. 11. Comparison of the neutron spectra for solid $\mathrm{UO}_{2}$ fuel (left) and UN FCM fuel (right) 

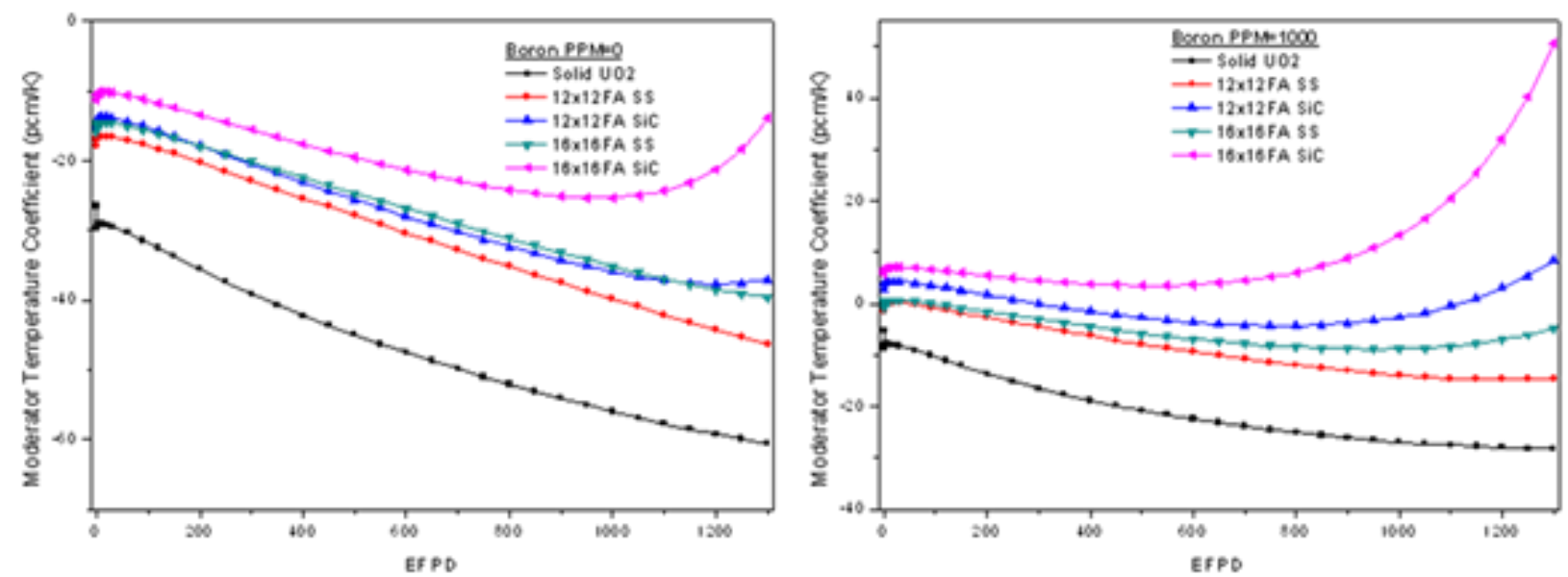

Fig. 12. Comparison of the MTC with soluble boron concentrations of 0 ppm (left) and $1000 \mathrm{ppm}$ (right)

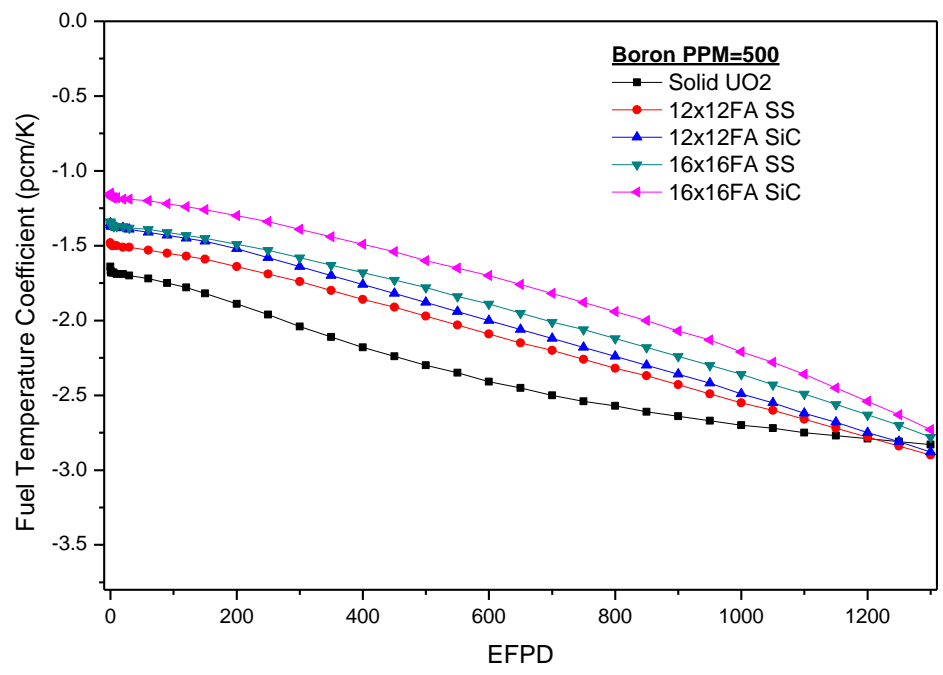

Fig. 13. Fuel temperature coefficients.

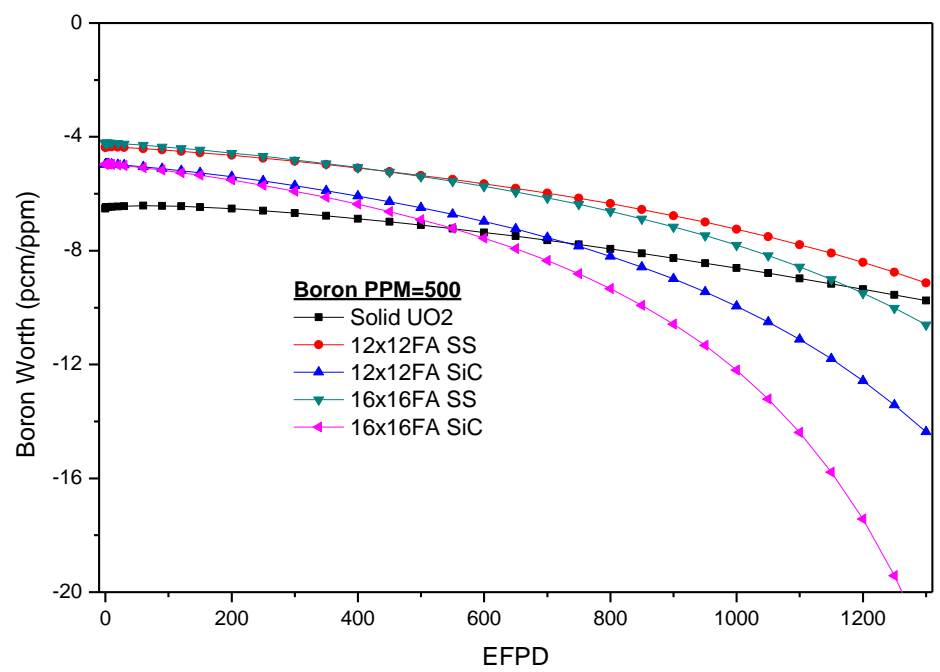

Fig. 14. Boron reactivity worth. 
The use of solid burnable poison is necessary to suppress the initial high neutron multiplication of FCM fuel due to its higher enrichment. Two candidate burnable poison elements were considered in this work: erbia and gadolinia. Natural gadolinia is used in the reference OPR-1000. Figure 15 compares the effect of burnable poisons for the UN FCM assembly. For gadolinia, it is suggested to use BISO-coated particles with gadolinia $\left(\mathrm{Gd}_{2} \mathrm{O}_{3}\right)$ kernels compacted with the TRISO fuel particle in the FCM fuel compact. The less-absorbing erbia $\left(\mathrm{Er}_{2} \mathrm{O}_{3}\right)$ burnable poison was also considered in this study. Natural erbia is an excellent burnable poison for FCM fuel, because it produces long-term effects on the reactivity with relatively small residual poison at the end of fuel life (Grossbeck et al., 2003). Erbia is mixed homogeneously in the SiC matrix of FCM compact, together with sintering agents alumina and yittria. Gadolinia on the other hand burns too fast whether it is homogeneously mixed in the matrix or even heterogeneously in BISO form. A 1\% volume fraction of erbia shows a reasonable reactivity containment and depletion performance. The optimal erbia concentration appears to be between $0.5-1 \%$. During the full-core loading pattern search, the adjustment of erbia concentration between $0.5-1 \%$ will be performed.

We also studied the case of introducing erbia burnable poison in the FCM fuel in the form of BISO particles. Figure 15 (b) displays various combinations of erbia kernels and packing fractions. It can be noted that there is only a small effect on the reactivity depletion behavior compared with the matrixmixed erbia cases.

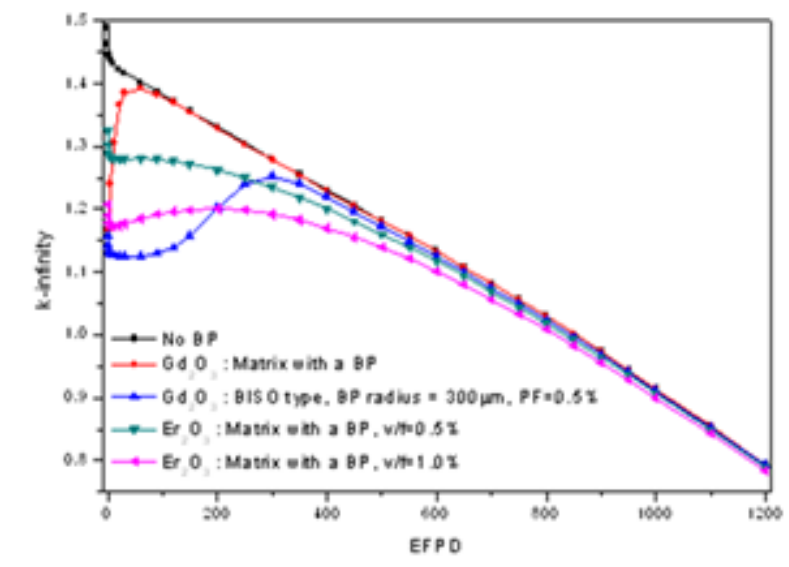

(a) $\mathrm{Gd}_{2} \mathrm{O}_{3} / \mathrm{Er}_{2} \mathrm{O}_{3}$ cases

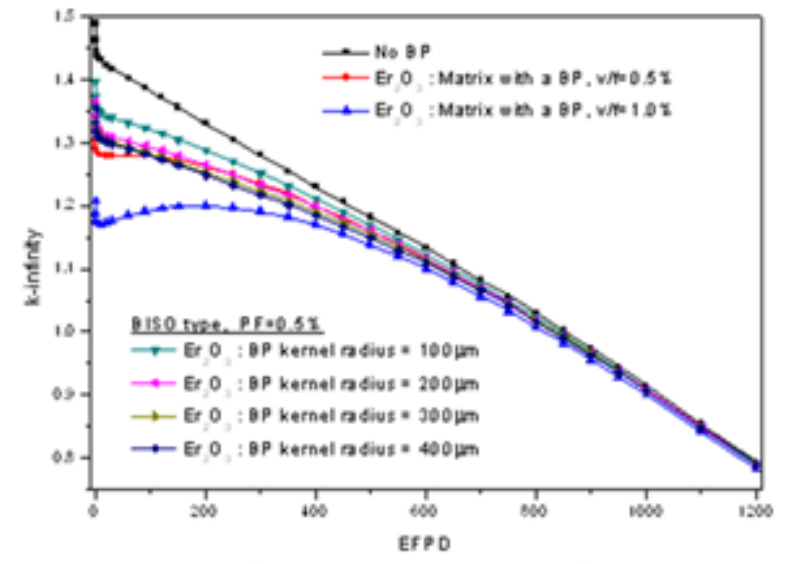

(b) $\mathrm{Er}_{2} \mathrm{O}_{3}$ cases

Fig. 15. Effects of burnable poisons in a UN FCM fuel assembly

Figure 16 shows the fast neutron fluence (above $0.18 \mathrm{MeV}$ neutron energy) in the HTR fuel compact, the LWR solid $\mathrm{UO}_{2}$ fuel pellet, and in the UN FCM fuel pellet and kernel. From the figure, it is evident that the fast neutron fluence of the LWR fuels is higher than that of the HTR fuel compact, due to the higher thermal power generation in the LWR. Figure 16 (a) shows that the fluence of the $12 \times 12$ UN FCM fuel pellet is nearly same as the solid $\mathrm{UO}_{2}$ fuel pellet and the fluence of the $16 \times 16 \mathrm{UN}$ FCM fuel pellet is slightly lower than that of the solid $\mathrm{UO}_{2}$ fuel pellet. Figure 16 (b) shows that the fluence in the fuel kernel of the $12 \times 12 \mathrm{UN}$ FCM fuel pellet is very much higher than that of the solid $\mathrm{UO}_{2}$ fuel pellet, and the fluence in the kernel of the $16 \times 16 \mathrm{UN}$ FCM is similar to the solid $\mathrm{UO}_{2}$ fuel pellet. The rapid increase in fluence after 800 EFPDs shows the effect of highly depleted fuel on the fluence in these assembly-level, constant-power calculations. It is likely to disappear in the analysis of full cores with multi-age fuel that will be performed next year. 


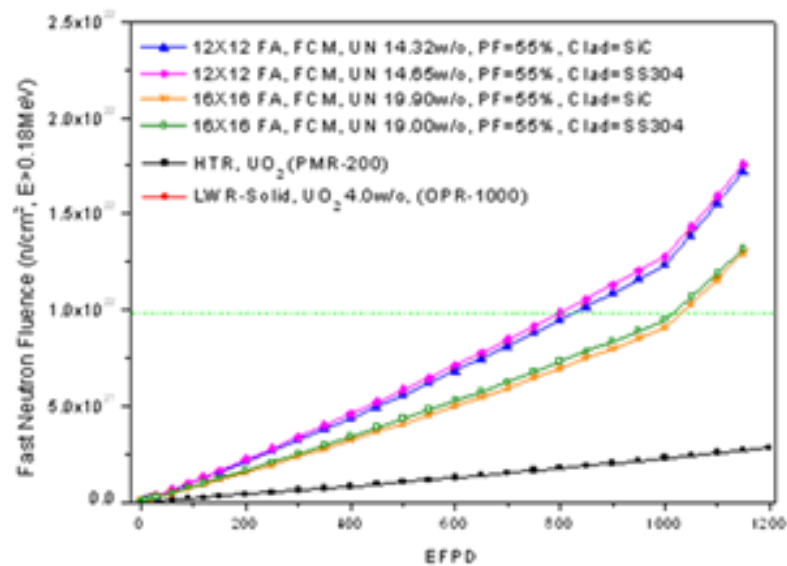

(a) at pellet average

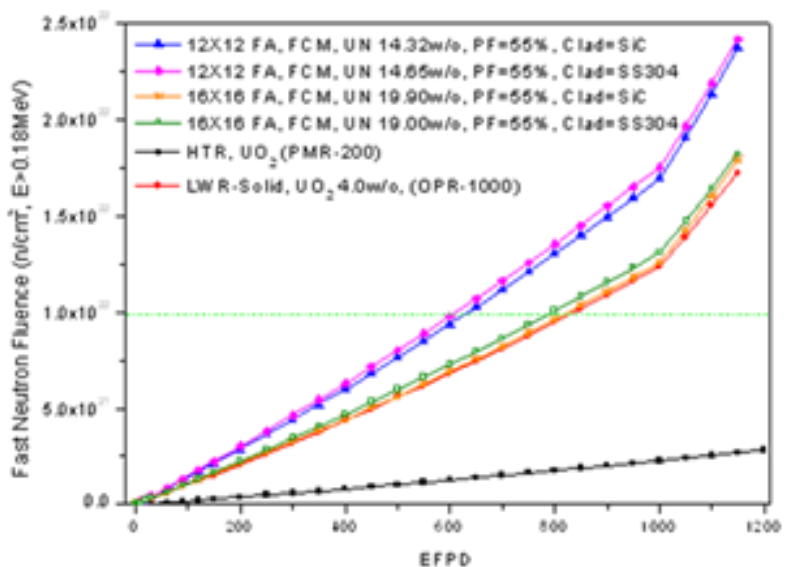

(b) at kemel average

Fig. 16. Comparison of the fast neutron fluence of the HTR fuel compact, LWR solid $\mathrm{UO}_{2}$ fuel, and UN FCM fuel pellet.

\subsection{FCM FUEL ASSEMBLY DESIGN FOR WESTINGHOUSE CORE}

The WH fuel assembly analysis was limited to the modeling of the $17 \times 17 \mathrm{WH}$ reference $\mathrm{UO}_{2}$ fuel assembly and to the specification of a $13 \times 13$ FCM UN fuel assembly of equivalent hydraulic diameter with the same initial fissile inventory. The reactivity/depletion curves were obtained for the $17 \times 17$ reference and $13 \times 13$ FCM assemblies along with the moderator temperature coefficient (MTC), the fuel temperature coefficient (FTC) and the void reactivity coefficient. Due to lack of funding, no attempt was made at optimizing the $13 \times 13 \mathrm{FCM}$ design.

The $17 \times 17$ WH configuration LWR fuel assembly modeled for this work contains 264 fuel pins, 24 guide tubes for control rods, and one central guide tube for instrumentation, which are arranged in a square lattice.

\subsubsection{Geometry Definition of 17×17 Reference Fuel Assembly}

Fuel pins, guide tubes, and overall dimensions of the $17 \times 17 \mathrm{WH}$ fuel assembly are summarized and shown in Figure 17. In this geometry description, the instrumentation and control rod guide tubes are completely filled with water. The compositions of the materials used are as follows.

- Fuel: $4 \%$ enriched $\mathrm{UO}_{2}$ fuel with $10.41 \mathrm{~g} / \mathrm{cm}^{3}$ density has been used in this fuel assembly, which is a typical enrichment for this standard configuration. Aside from fuel density and fuel composition, it is necessary to define its temperature in order to work with accurate cross sections values during the simulation. According to the temperature distribution over the section of the pellet, an average temperature of $1200 \mathrm{~K}\left(927^{\circ} \mathrm{C}\right)$ has been considered.

- Water: water enters the vessel at $\sim 560 \mathrm{~K}$ and exits at $\sim 590 \mathrm{~K}$ temperature, so according to these water work temperatures, an average temperature of $573 \mathrm{~K}\left(300^{\circ} \mathrm{C}\right)$ has been considered for the simulation, with a density of $725.55 \mathrm{~kg} / \mathrm{m}^{3}$.

- Clad: Zirlo is the zirconium alloy used for the cladding material, whose density is $6.5 \mathrm{~g} / \mathrm{cm}^{3}$ and composition is summarized in the figure below. A clad temperature of $600 \mathrm{~K}$ was used. 


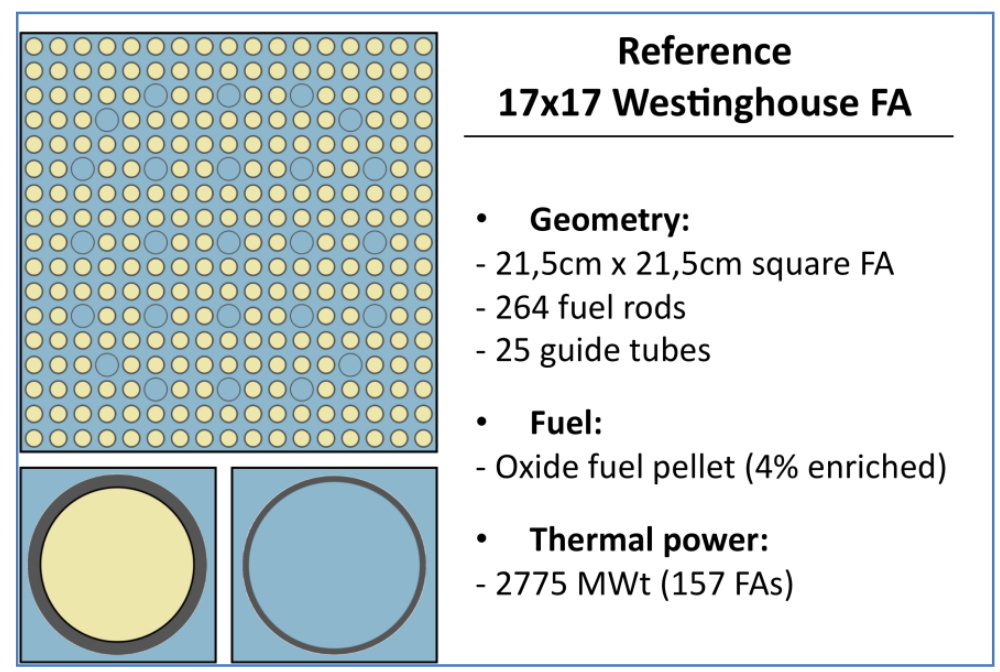

Fig. 17. Westinghouse $17 \times 17$ reference fuel geometry.

\section{Boundary Conditions}

- A periodic boundary condition for the fuel assembly external surfaces. Using this option, when the neutron encounters a periodic boundary, it is moved to the opposite surface of the geometry. This is an adequate boundary condition because of working at a fuel assembly level, which could receive neutrons from the surrounding fuel assemblies.

- A quadrant symmetry condition for the overall fuel assembly geometry. Statistical errors and time of simulation can be reduced by taking advantage of the symmetry condition, which divides the desired universe into similar portions, simplifying the treated geometry.

\subsubsection{Geometry Definition of $13 \times 13$ FCM Fuel Assembly}

A new fuel assembly was devised in order to develop a feasible fuel replacement for the $17 \times 17 \mathrm{WH}$ configuration. The new fuel assembly uses FCM uranium nitride (UN) fuel instead of $\mathrm{UO}_{2}$ fuel and has compatible characteristics with the reference $17 \times 17$ fuel assembly, in terms of power generation, thermohydraulics and neutronics. Initial design screening criteria are shown in Figure 18.

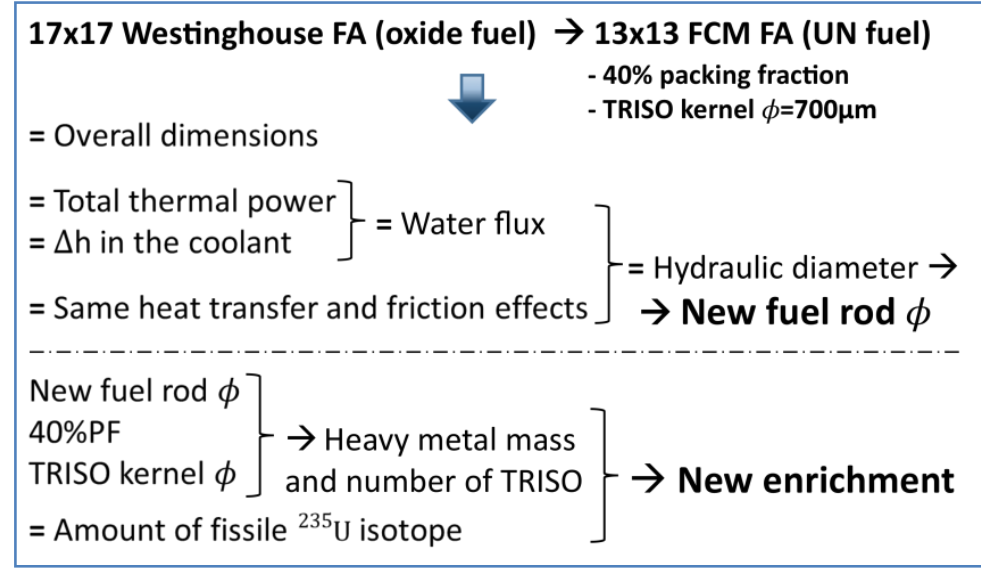

Fig. 18. Screening of fuel assembly design. 
The $13 \times 13$ FCM fuel assembly uses UN fuel because the density of UN is significantly higher than that of the alternatives (40-50\% higher than the $\mathrm{UO}_{2}$ fuel and 30-40\% higher the UCO fuel), allowing lower enrichment requirements. However, while $\mathrm{UO}_{2}$ and UCO kernels with TRISO coatings have been successfully produced for years, UN kernels are still in development.

Thanks to the higher conductivity of the FCM fuel, a smaller number of wider fuel rods can be used in the new fuel assembly compared to the original, which is an advantage for two reasons.

- A larger diameter of the compact provides better self-shielding, which should increase cycle length and reduce the need for burnable poisons, especially at the beginning of the cycle. This is very important since the new FCM-based configuration uses higher enrichment fuels.

- A larger diameter of the compact will allow higher TRISO particles packing fractions by decreasing the wall effect. This is important in order to increase fuel pin linear power density without increasing fuel enrichment.

After fixing the number of fuel rods for the new fuel assembly and the type of fuel used in it, fuel rod diameter, pellet packing fraction, and the UN enrichment for the $13 \times 13$ FCM fuel assembly were calculated, in order to obtain similar thermo-hydraulic and power characteristics as the reference $17 \times 17$ fuel assembly configuration.

The diameter of the fuel rods in the $13 \times 13$ fuel assembly was calculated by keeping the heat transfer capability of the fuel assemblies the same. Therefore the hydraulic diameter was kept constant so that for the same fluid mass flow, the same pressure drop, and same heat transfer are obtained in the $13 \times 13$ as in the $17 \times 17$ fuel assemblies.

The pellet diameter for the $13 \times 13$ fuel assembly was derived as $1.242 \mathrm{~cm}$, using a $0.053 \mathrm{~cm}$ clad thickness and $0.0085 \mathrm{~cm}$ gap.

The packing fraction, enrichment, and diameter of the fuel kernel were determined by power compatibility considerations. As a first guess, the initial amount of fissile material contained in the fuel assemblies was kept constant.

Since the $13 \times 13$ configuration uses FCM UN fuel instead of solid $\mathrm{UO}_{2}$ fuel, the $\mathrm{UN}$ enrichment will strongly depend on the TRISO particle geometry and compact configuration. Particularly, it will depend on the TRISO kernel diameter (KD) and packing fraction parameter (PF).

The theoretical packing fraction of uniform-sized spheres approaches 74\%; however, during the compacting process, practical considerations reduce the maximum achievable packing fraction to 50 $60 \%$, with $45 \%$ routinely obtained in test runs.

The PF-KD combination defines two important parameters for the FCM fuel rod configuration, as shown in Figure 19. The combination fixes the number of TRISO particles inside the fuel pin and also fixes the total UN fuel volume, which in turn determines the fissile material amount, through the third variable, the enrichment. 


\begin{tabular}{|c|c|c|c|c|c|c|}
\hline \multicolumn{7}{|c|}{ Number of TRISO particles } \\
\hline KD / PF & \multicolumn{2}{|c|}{$30 \%$} & $35 \%$ & $40 \%$ & $45 \%$ & $50 \%$ \\
\hline $400 \mu \mathrm{m}$ & \multicolumn{2}{|c|}{810.647} & 945.755 & 1.080 .862 & 1.215 .970 & 1.351 .078 \\
\hline $500 \mu \mathrm{m}$ & \multicolumn{2}{|c|}{537.124} & 626.645 & 716.165 & 805.686 & 895.207 \\
\hline $600 \mu \mathrm{m}$ & \multicolumn{2}{|c|}{374.033} & 436.372 & 498.711 & 561.050 & 623.389 \\
\hline $700 \mu \mathrm{m}$ & \multicolumn{2}{|c|}{270.820} & 315.956 & 361.093 & 406.229 & 451.366 \\
\hline \multicolumn{7}{|c|}{ UN enrichment (\%) } \\
\hline \multicolumn{2}{|c|}{ KD / PF } & $30 \%$ & $35 \%$ & $40 \%$ & $45 \%$ & $50 \%$ \\
\hline \multicolumn{2}{|c|}{$400 \mu \mathrm{m}$} & 31,29 & 26,82 & 23,46 & 20,86 & 18,77 \\
\hline \multicolumn{2}{|c|}{$500 \mu \mathrm{m}$} & 24,18 & 20,72 & 18,13 & 16,12 & 14,51 \\
\hline \multicolumn{2}{|c|}{$600 \mu \mathrm{m}$} & 20,09 & 17,22 & 15,07 & 13,39 & 12,05 \\
\hline \multicolumn{2}{|c|}{$700 \mu \mathrm{m}$} & 17,47 & 14,98 & 13,11 & 11,65 & 10,48 \\
\hline
\end{tabular}

Fig. 19. Ranges of packing fraction and enrichment.

The advantage of a small KD is the possibility of achieving a higher burnup than using larger diameters by better neutron moderation and lower self-shielding. However, a small KD will significantly decrease the cycle length, increase the absorbent requirements to keep the system critical at BOL, and will require a higher UN enrichment to reach the same amount of fissile material as the reference configuration.

Taking into account that burnup is already highly increased by using FCM fuel instead of $\mathrm{UO}_{2}$ regardless of the KD and the PF, a large KD was selected for the FCM configuration: $\mathrm{KD}=700 \mu \mathrm{m}$.

A well-established packing fraction value of $40 \%$ was selected as the initial-guess value for the design of the $13 \times 13$ FCM fuel assembly. Using these values, the obtained enrichment requirement that would give the same amount of initial fissile material in the $13 \times 13$ FCM UN fuel assembly as in the $17 \times 17$ solid $\mathrm{UO}_{2}$ assembly is about $13 \%$, below the $20 \%$ proliferation level limit. Figure 20 summarizes the selected fuel assembly design parameters. 

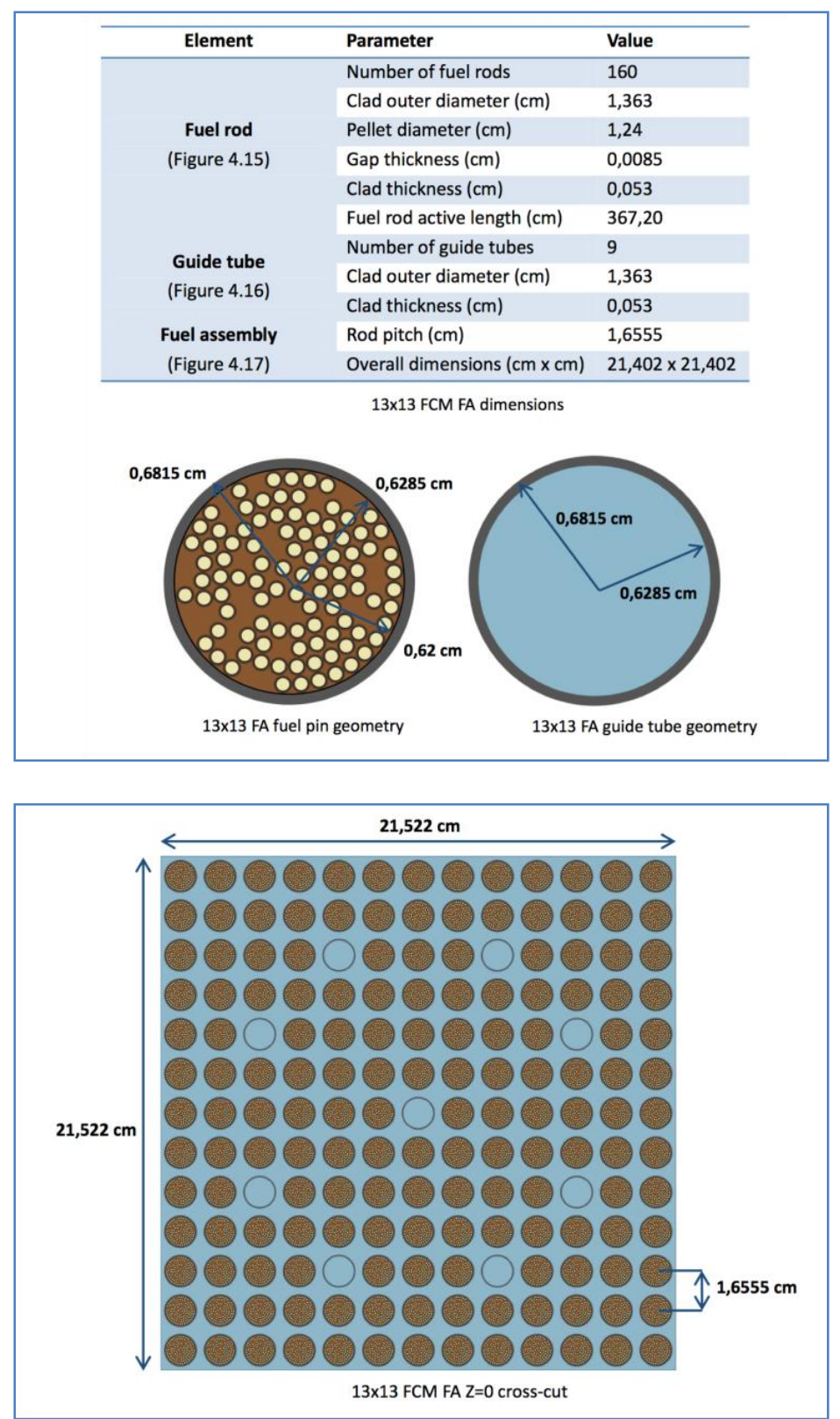

Fig. 20. $13 \times 13$ fuel assembly design.

\subsubsection{3×13 FCM Fuel Assembly Initial Design Analysis}

The $13 \times 13$ FCM fuel assembly is a square-shaped fuel assembly with the same overall dimensions as the $17 \times 17$ configuration, a necessary condition to maintain the dimensions of the whole core. The new fuel assembly contains 160 fuel pins and nine guide tubes. The guide tube dimensions are approximated to be the same as the fuel pin dimensions in the simulation, which is not relevant regarding the neutronics, since the tubes are filled with water. 


\section{Materials Definition}

FCM Fuel: The fuel consists of TRISO particles with UN kernels $\left(14.32 \mathrm{~g} / \mathrm{cm}^{3}\right.$ density) embedded in a $\mathrm{SiC}$ matrix $\left(3.18 \mathrm{~g} / \mathrm{cm}^{3}\right.$ density). In addition, and according to the temperature distribution over the section of the pellet, a simulation temperature of $600 \mathrm{~K}$ has been considered because of the low operation temperature gradient achieved by the UN higher thermal conductivity. TRISO material composition is given in Figure 21.

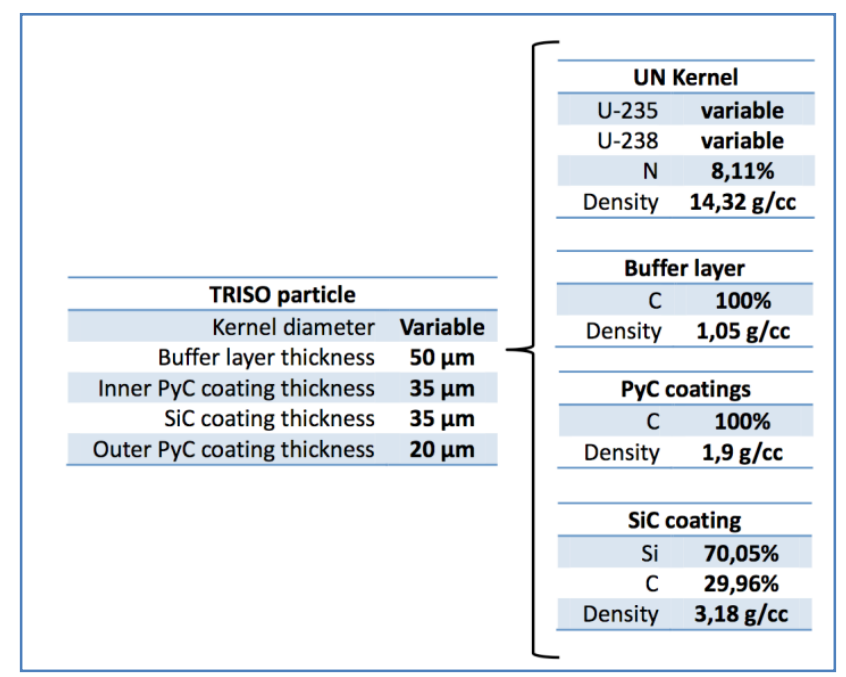

Fig. 21. TRISO material composition.

Clad and Water: Clad and water are the same as in the original $17 \times 17 \mathrm{WH}$ fuel assembly. First, the water is at the same temperature and the same enthalpy rise is experienced in both configurations. Secondly, although the use of a SiC clad would be possible by using FCM fuel, a Zirlo clad will be simulated; it should be noted that this differs from the OPR-1000 analysis and does not fully conform to the goals of accident tolerant fuels but is done in order to focus the results on fuel configuration aspects

The periodic boundary condition for the fuel assembly external surfaces and the thermal scattering cross section used for the hydrogen in water are also included in the new configuration.

The SERPENT code was used to perform reactivity and depletion calculations on the $17 \times 17$ reference and the $13 \times 13$ FCM replacement configurations.

\section{Analysis of Reactivity Calculations}

As a first comparison of the $17 \times 17 \mathrm{WH} \mathrm{UO}_{2}$ fuel assembly and the initial design of the $13 \times 13 \mathrm{FCM}$ fuel assembly, a reactivity calculation was performed along the fuel cycle. The analysis for the reference $17 \times 17$ configuration is run from the steady state calculation until fuel is typically discharged from the reactor (i.e., $50 \mathrm{MWd} / \mathrm{kgHM}$ ), whereas the FCM fuel assembly calculation is run until the typical discharge value of the LEU TRISO particles in the HTGR (i.e., $120 \mathrm{MWd} / \mathrm{kgHM}$ ).

Fuel assembly reactivity variation with burnup is given in Figure 22 . The initial very rapid $\mathrm{k}_{\text {eff }}$ decrease is due to the fast accumulation of generated fission products such as ${ }^{135} \mathrm{Xe}$ or ${ }^{149} \mathrm{Sm}$, which act as neutron absorbers and decrease $\mathrm{k}_{\text {eff }}$. 
At BOL, significantly higher $\mathrm{k}_{\mathrm{eff}}$ values are obtained from the FCM fuel configuration for two reasons: the higher fuel enrichment and the inert SiC matrix around TRISO particles lead to a significant lack of self-shielding in the FCM fuel, and the significant amount of carbon in the TRISO particles layers and the $\mathrm{SiC}$ matrix in FCM fuel act as a moderator and enhance the thermalization process. This effect combined with a lower amount of ${ }^{238} \mathrm{U}$ in the fuel leads to an increase in the resonance escape probability $(\rho)$, increasing $\mathrm{k}_{\mathrm{eff}}$ significantly.

Plotting the same data as a function of the Effective Full Power Days (EFPDs), the FCM fuel depletion rate is very stiff compared to the $\mathrm{UO}_{2}$ reference fuel, reaching a lower $\mathrm{k}_{\text {eff }}$ after 700 days. This behavior is not expected at first because both fuel assemblies must produce the same total power and they start from the same amount of fissile material. As shown in Figure 23, for the reference oxide fuel, a larger fraction of the energy comes from the fission of some transuranic elements (TRU) such as ${ }^{239} \mathrm{Pu}$ and ${ }^{241} \mathrm{Pu}$ due to the higher quantity of ${ }^{238} \mathrm{U}$ in the oxide fuel composition, which reduces the resonance escape probability and increases its neutron capture rate, leading to breed more plutonium than the FCM fuel and to a longer cycle length.
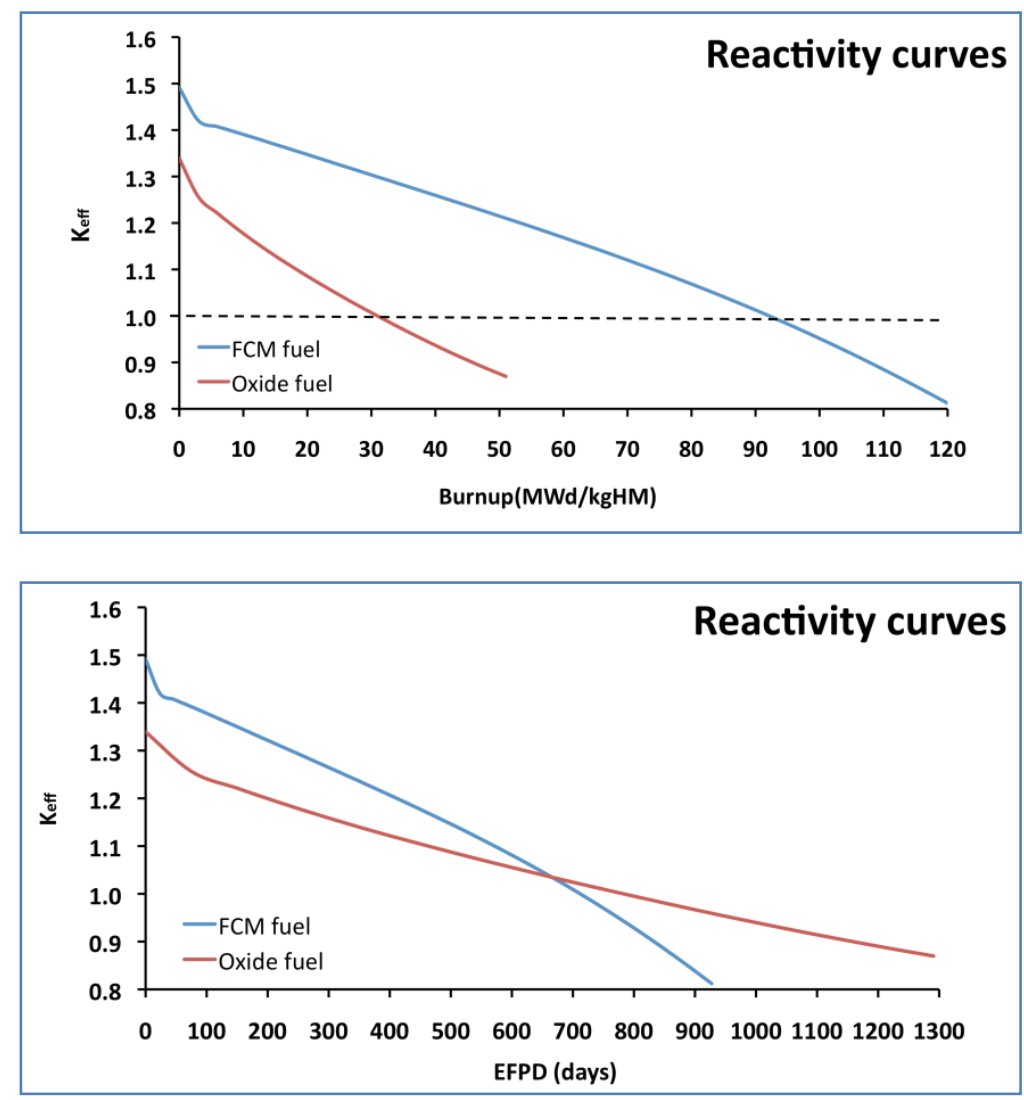

Fig. 22. Core reactivity. 


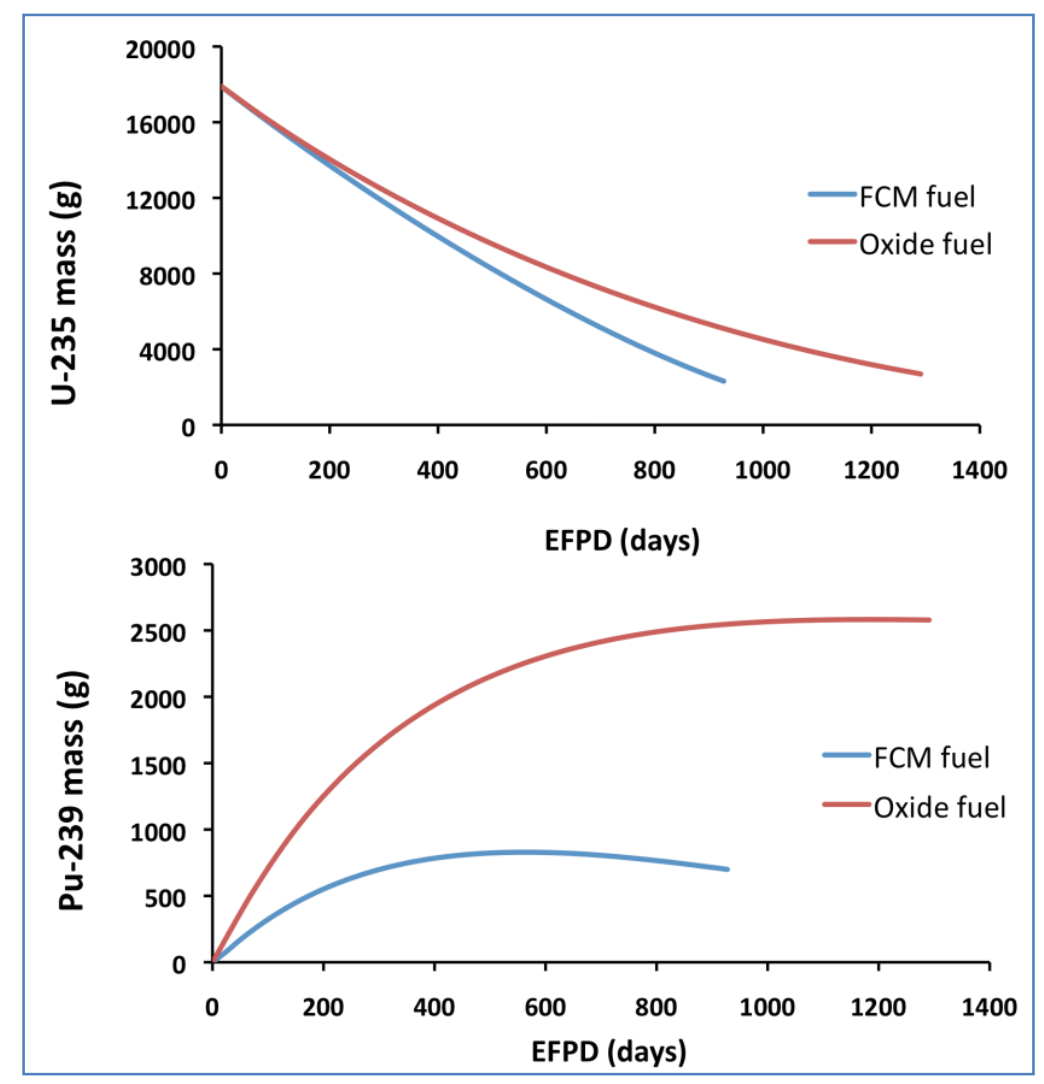

Fig. 23. Inventory of fissile material.

Another effect of the lack of ${ }^{238} \mathrm{U}$ is that many neutrons are absorbed preferentially into parasitic fission products rather than in fertile ${ }^{238} \mathrm{U}$, and therefore the reactivity decreases more quickly. This situation is conservatively assessed in the assembly-level calculations, but it will be improved to some extent in the full core analysis, where different ages of fuel reside in the reactor core at the same time. At this stage however, it is safe to say that the assumption of constant fissile inventory to ensure the same power production over time (similar fuel cycle length) is not correct and that in order to match the cycle lengths of the reference $17 \times 17$ reference fuel assembly and the $13 \times 13$ FCM assembly, either the enrichment or the packing fraction of the FCM fuel will have to be increased over the initial guess reported here.

\section{Moderator Temperature Coefficient}

The Moderator Temperature Coefficient (MTC) is the first safety parameter to be analyzed. MTC measures the change in reactivity caused by a change in moderator temperature. Figure 24 shows the MTC for FCM and reference solid $\mathrm{UO}_{2}$ fuel assemblies. 


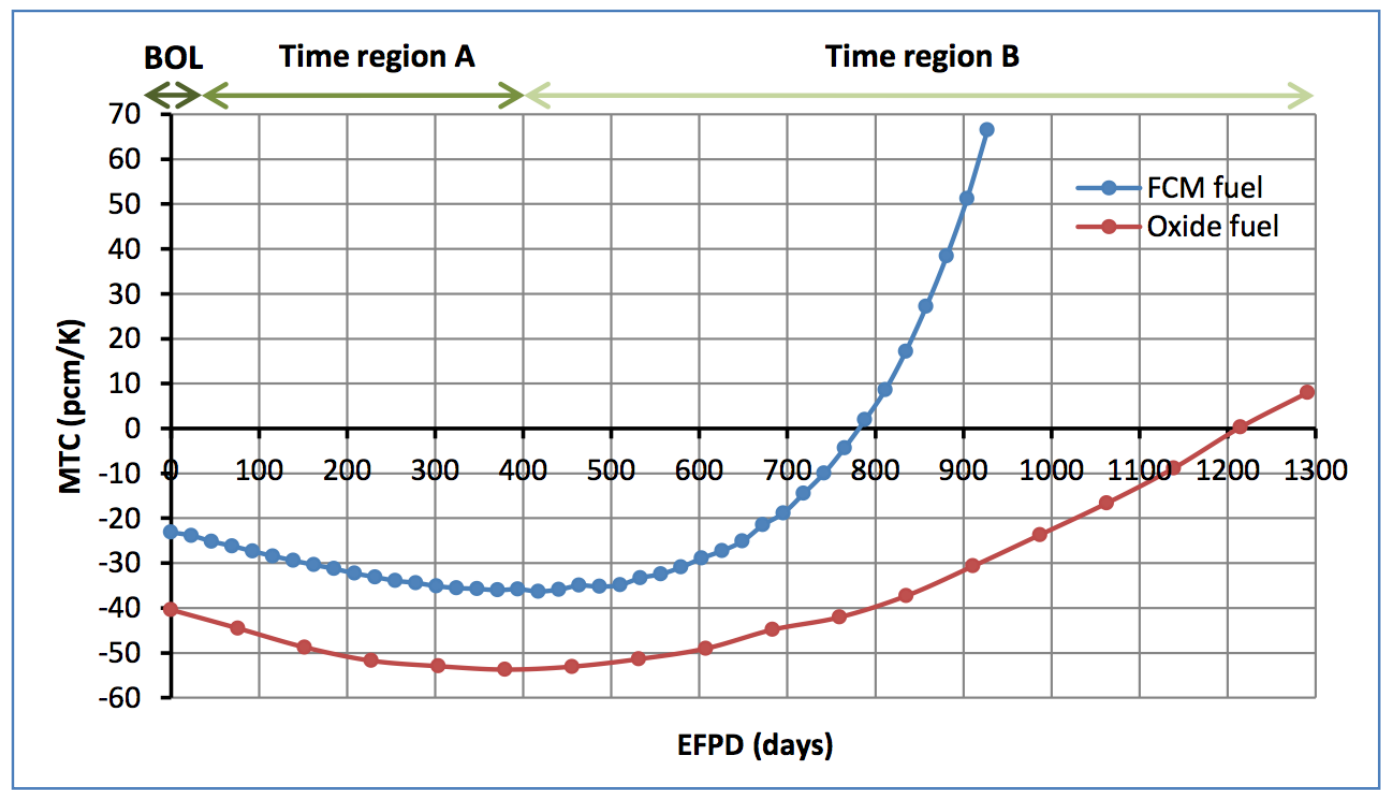

Fig. 24. Moderator temperature coefficient.

BOL. MTC behavior at BOL is negative in both cases $\left(17 \times 17 \mathrm{UO}_{2}\right.$ reference and $\left.13 \times 13 \mathrm{FCM}\right)$ due to the expected neutron spectrum move towards higher energies, hence increasing resonance absorption and decreasing $\mathrm{k}_{\mathrm{eff}}$ value. However, FCM fuel achieves a less negative MTC value compared to the reference oxide fuel. This happens because the decrease in moderation is not as significant in the FCM fuel case due to the already softer spectrum caused by the large amounts of carbon in the core, which act as a neutron moderator. In this sense, FCM fuel configuration becomes less sensitive to changes in water temperature because neutron thermalization is shared between the water and the carbon in the fuel.

Time region A. Time region A corresponds to the first 400 days of cycle, where MTC becomes more negative in both cases. This is due to the depletion of ${ }^{235} \mathrm{U}$, which increases the ${ }^{238} \mathrm{U}-\mathrm{to}^{-}{ }^{235} \mathrm{U}$ ratio and thus enhances ${ }^{238} \mathrm{U}$ resonance absorption. This leads to a larger decrease of the resonance escape probability $(\rho)$ when water temperature increases, leading to a more negative MTC value.

Time region B. The MTC values become less negative and even positive as EOL is approached. This is due to the accumulation of absorbent fission products, which become more important for high burnup, and the reduction of resonant absorbers (e.g., $\left.{ }^{235} \mathrm{U}\right)$.

A significant difference between both configurations is noticed since FCM fuel tends to a positive MTC value faster than the oxide fuel. This behavior could be explained by two reasons.

- The resonance absorptions are not only due to ${ }^{238} \mathrm{U}$ but also to ${ }^{240} \mathrm{Pu}$, especially near EOL. The amount of ${ }^{240} \mathrm{Pu}$ becomes significantly higher by using oxide fuel since more ${ }^{239} \mathrm{Pu}$ is generated; therefore, the negative contribution of ${ }^{240} \mathrm{Pu}$ to the MTC does not allow the oxide fuel reference configuration to reach a positive value at EOL as fast as in FCM fuel, as shown in Figures 25.

- There is a significant difference between the two configurations regarding the ${ }^{\text {fission product }}$ absorption contributions. For high burnup values, a significant fraction of the fuel has been depleted compared to BOL, which increases the moderator-to-fuel ratio and thus makes the positive contribution of fission product absorption to the MTC more significant at EOL, particularly in the case of the FCM fuel because its configuration leads to a higher depletion rate, which significantly thermalizes the neutron spectrum at high burnups compared with oxide fuel. 


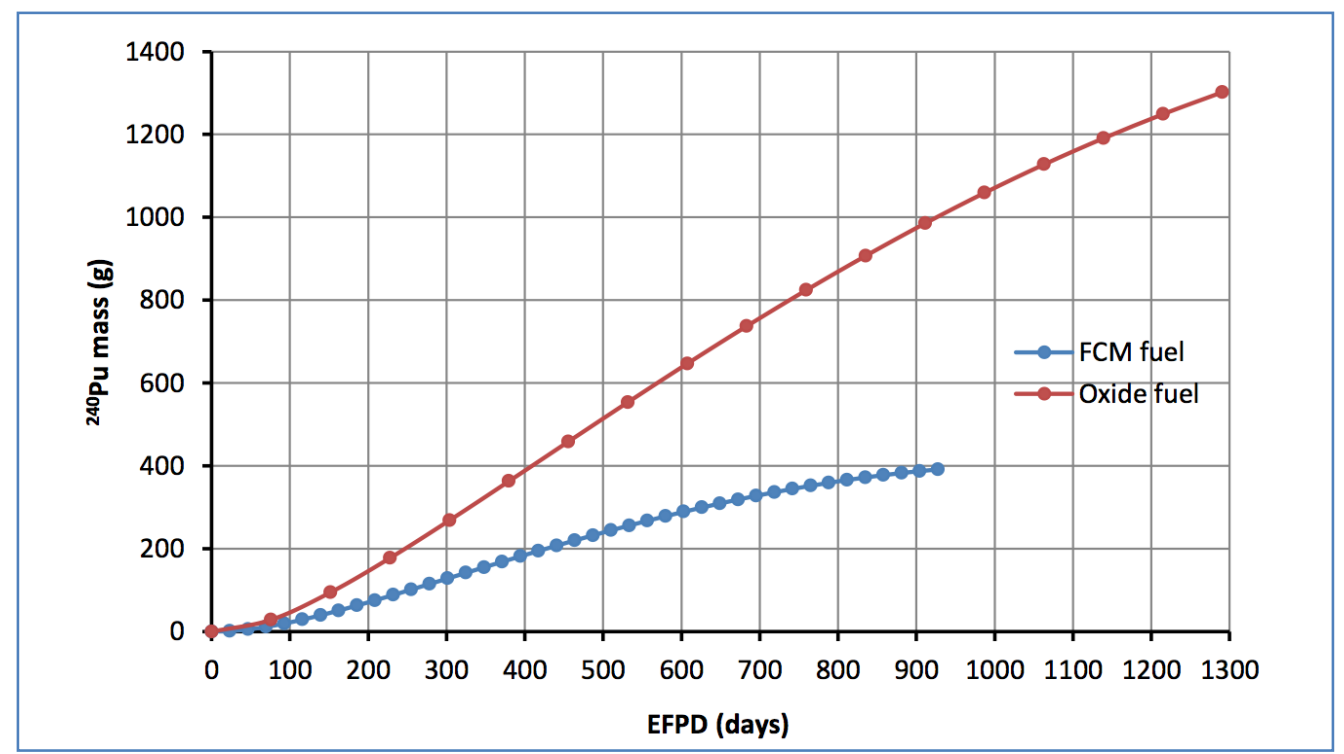

Fig. 25. Mass of ${ }^{240} \mathrm{Pu}$.

A very useful parameter in order to see these effects on the MTC curves is the thermal utilization factor (f), which represents the fraction of neutrons absorbed by the fuel over the total neutrons absorbed in the system. It is observed that thermal utilization factor values decrease much faster for the FCM fuel than for the oxide fuel due to the higher depletion rates of ${ }^{235} \mathrm{U}$, as shown in Figure 26.

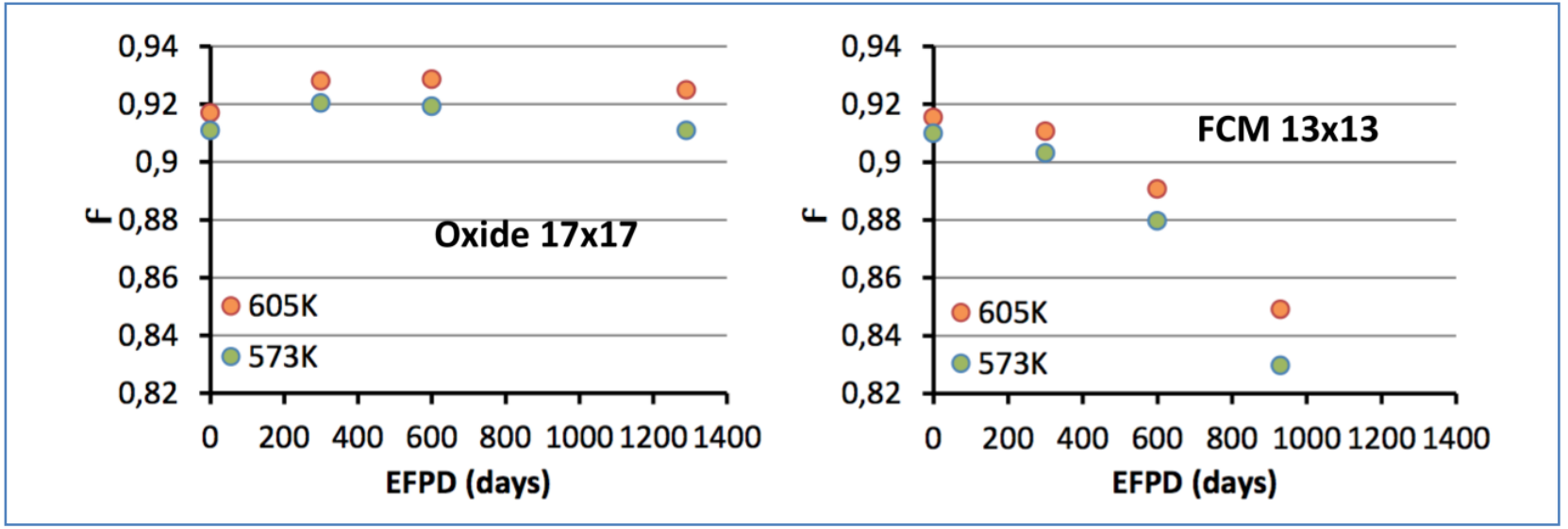

Fig. 26. Thermal utilization factor.

It is clear from these results of the MTC analysis that the MTC of the FCM fuel assembly becomes much more positive at EOL than for the reference $\mathrm{UO}_{2}$ assembly; however, this appears to be largely caused by the excessive depletion of fissile material in the FCM fuel at extended burnups and should decrease substantially for an FCM fuel with more balanced burnup.

\section{Fuel Temperature Coefficient}

The fuel temperature coefficient (FTC) has been calculated similarly to the MTC, but in this case by running various simulations changing only the fuel temperature (i.e., $300 \mathrm{~K}, 600 \mathrm{~K}, 900 \mathrm{~K}, 1200 \mathrm{~K}$, $1500 \mathrm{~K}$ and $1800 \mathrm{~K}$ ). Figure 27 shows the calculated FTC. 


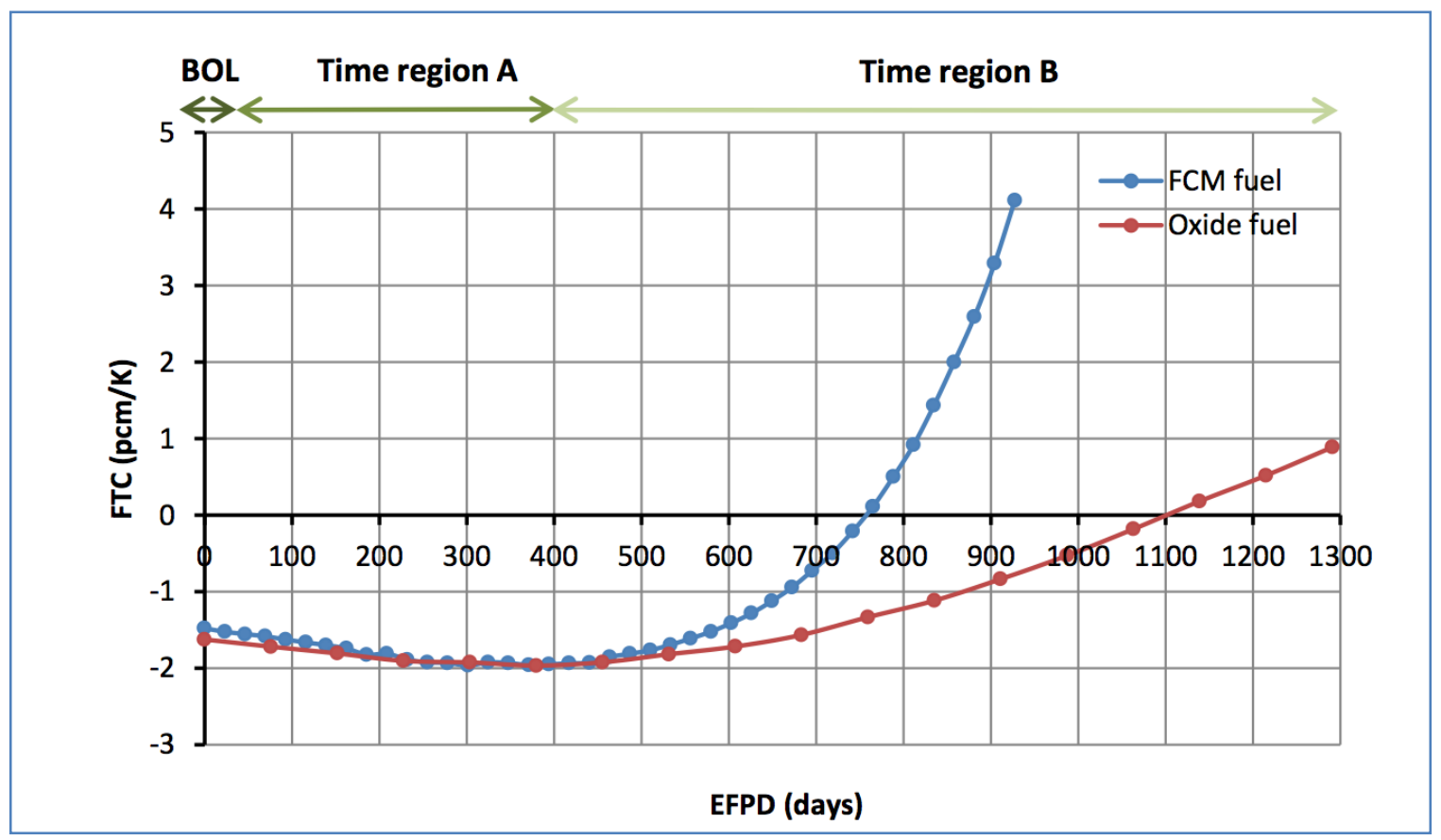

Fig. 27. Fuel temperature coefficient.

BOL: The FTC presents negative values for both configurations, similarly to the MTC behavior, because the increasing fuel temperature also shifts the Maxwell neutron spectrum to higher energies. This is due to the increase in resonance capture probability by a widening in the ${ }^{238} \mathrm{U}$ absorption resonances (Doppler Effect), which leads to a decrease in the resonance escape probability $(\rho)$, reducing the number of thermalized neutrons and thus hardening neutron spectrum. On the other hand, it can be observed that the behavior of both fuel assemblies is much more similar for the FTC than for the MTC.

Time region A: FTC becomes more negative in both cases due to the depletion of ${ }^{235} \mathrm{U}$, which increases the ${ }^{238} \mathrm{U}-$ to- $^{235} \mathrm{U}$ ratio.

Time region B: As seen with MTC results, FTC values become less negative and even become positive when approaching EOL due to fission product accumulation and relative reduction in heavy metal resonance absorbers. This behavior differs from the OPR-1000 results.

In general, the absolute values of FTC are significantly lower than those of MTC. However, this does not reduce the importance of FTC because FTC is the first mechanism to act after a sudden increase in the power, so a consistently negative FTC value (however small) is essential in regard to safety.

\section{Void Reactivity Coefficient}

The void reactivity coefficient (CVR) is calculated by the difference between the $\mathrm{k}_{\mathrm{eff}}$ obtained from a standard system configuration with coolant and a completely voided system. The CVR calculation is interesting because it is a useful parameter to describe the change in the reactivity of a reactor after a LOCA, when water is removed from the core. However, to perform a good CVR analysis, it is necessary to take into account the neutron leakage factor, which becomes essential when there is no moderator in the core. For the CVR analysis at the fuel assembly level, the reflective boundary condition (no leakage) 
has been maintained, so the results must be taken as a comparison of the two fuel assemblies, as shown in Figure 28.

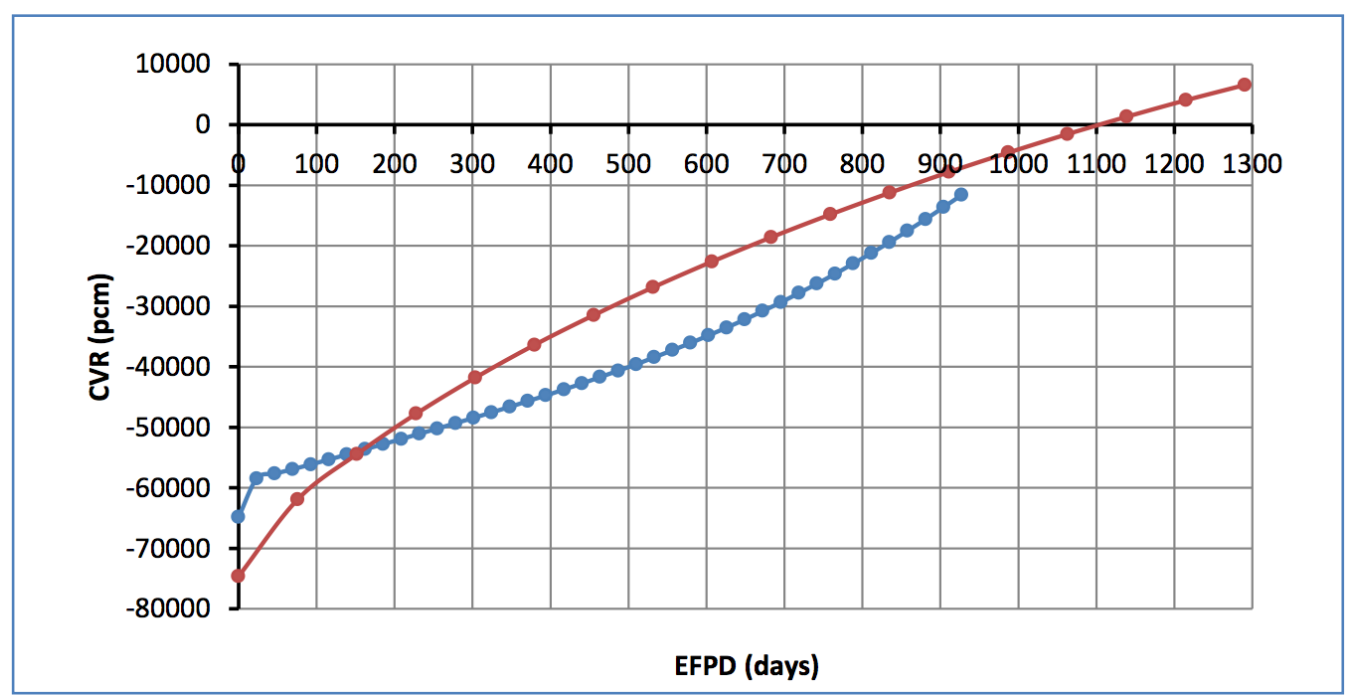

Fig. 28. Void reactivity coefficient.

At BOL, it can be observed that CVR is less negative for the FCM fuel configuration. In fact, when the core is completely voided, the carbon of the FCM fuel still maintains some moderation. As time passes, CVR becomes less negative in both cases.

The CVR behavior of both fuel assemblies is a result of the difference between the respective $\mathrm{k}_{\text {eff }}$ values with and without water in the core; as shown in Figure 29, the FCM fuel curve without water decreases with burnup, whereas the oxide fuel curve increases significantly. This is an interesting behavior that needs to be confirmed by full core analysis.

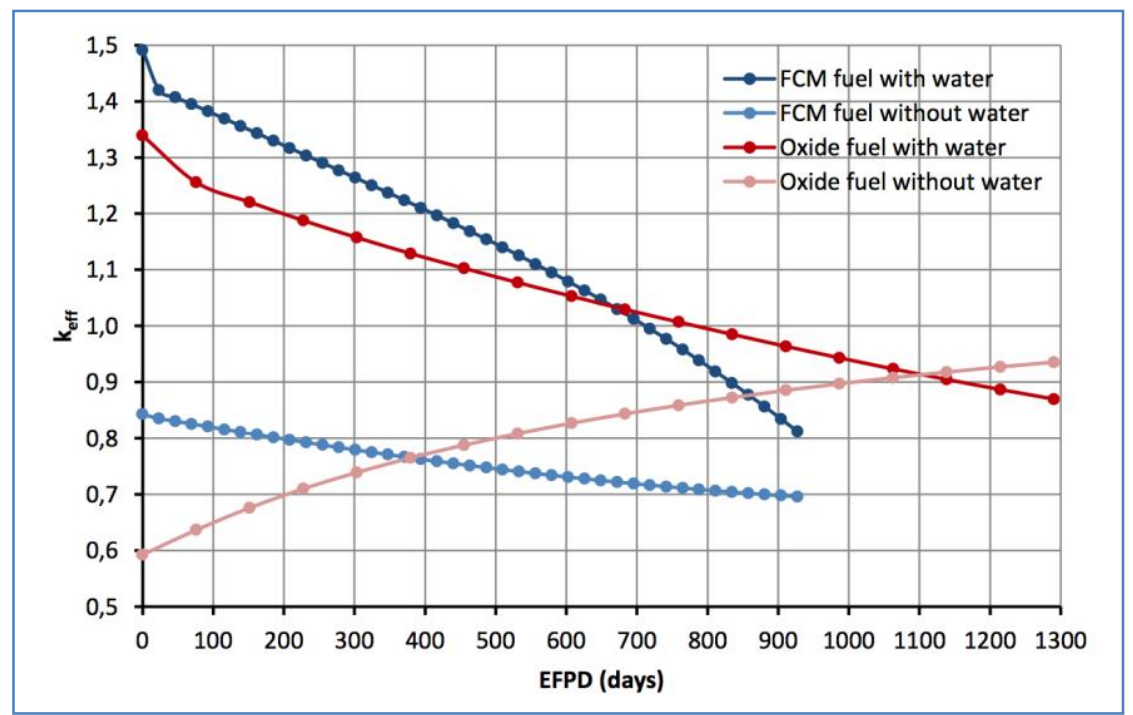

Fig. 29. Reactivity with and without water. 


\subsubsection{Summary of Results}

The results presented here are in partial fulfillment of the $17 \times 17 \mathrm{WH}$ fuel assembly tasks outlined in the work plan.

The limited amount of analysis done on the $13 \times 13$ assembly replacement for the $17 \times 17 \mathrm{WH}$ fuel shows agreement on most issues with the analogous and more extensive work performed on the $12 \times 12$ FCM fuel replacement of the 16×16 System-80 (OPR-1000) fuel assembly.

The use of FCM fuel increases enrichment requirements due to the geometry of the TRISO particles and the surrounding $\mathrm{SiC}$ matrix. The required enrichment increase is dependent on the packing fraction value and the size of the TRISO particles. A simple invariance of the fissile fuel inventory is not sufficient and will yield shorter fuel cycles, because the burnup of the higher enrichment FCM fuel is faster.

The SERPENT code has proven to be a useful tool in modeling the geometry of the fuel assembly and modeling TRISO particles inside the desired matrix.

Regarding the reactivity curves study, the burnup simulations have shown that FCM fuel increases significantly the reactivity introduced by the fuel at BOL, basically due to its higher enrichment, the SiC neutron transparency, and the extra thermalization factor of the carbon. However, the use of a lower amount of total heavy metal mass compared to oxide fuel decreases the breeding of ${ }^{239} \mathrm{Pu}$ and ${ }^{241} \mathrm{Pu}$ isotopes, leading to deplete faster the ${ }^{235} \mathrm{U}$ isotope, although producing the same total amount of energy. This characteristic is disadvantageous to the neutron economy because of decreasing significantly the cycle length, and therefore the packing fraction or the enrichment needs to be increased more than necessary to match the initial fissile inventory.

Regarding the MTC, the use of FCM fuel gives a less negative feedback during the first half of cycle compared to oxide fuel due to the presence of significant amounts of carbon in its composition, which counteracts the loss of moderation capability after an increase in water temperature. Just as in the $12 \times 12$ analysis, the MTC positive behavior of the $13 \times 13$ FCM fuel assembly appears at the EOL only, which for the case analyzed is well beyond the point where the fuel would have to be extracted from the core because of lack of reactivity. The FTC behavior of the $13 \times 13$ FCM and the $17 \times 17$ reference cases is much more similar, indicating no major issues.

Finally, the CVR analysis shows a safer behavior for the $13 \times 13$ FCM fuel with respect to the $17 \times 17$ reference. This could be important in the analysis of LOCA events. However, the full core analysis is required to properly take into account core leakage effects in the analysis of the CVR.

\subsection{PLANNED ACTIVITIES FOR NEXT YEAR}

\subsubsection{FCM Fuel and Core Design for OPR-1000}

In 2013 Task 1 will perform the core design analysis of the UN FCM fuel-loaded OPR-1000 by using the fuel assembly analysis results. The full-core analysis will provide the cycle-by-cycle core reloading performance, including the fuel assembly loading patterns, cycle length, and accurate behavior of reactivity coefficients and other safety parameters. In addition, a more complete analysis will be performed to assess and address the issue of fast neutron fluence in the FCM-fueled core. 


\subsubsection{FCM Fuel and Core Design for Westinghouse Plants}

In 2013 Task 1 will complete the assembly-level analysis of the UN FCM fuel and start the core design analysis of the UN FCM fuel-loaded WH 1000 by using the fuel assembly analysis results. The full-core analysis will provide the cycle-by-cycle core reloading performance, including the fuel assembly loading patterns, cycle length, and behavior of reactivity coefficients, and other safety parameters.

\subsection{SUMMARY}

From the preliminary neutronics exploration of FCM replacement fuel assemblies for OPR-1000 and WH cores, the following was accomplished.

\section{OPR-1000 Core}

The DeCART2D/MASTER code system for the FCM fuel-loaded assembly and core design analysis has been established.

The fuel assembly depletion calculations have been performed for the FCM fuel-loaded assemblies consisting of $12 \times 12$ and $16 \times 16$ fuel assembly grids. The analysis compared the FCM fuel assembly with the reference solid fuel pin assembly consisting of the $16 \times 16$ fuel assembly grid.

The SS304 and SiC for the cladding material in fuel rod have been investigated for the UN FCM fuel.

From the scoping analysis and fuel assembly depletion calculations, the optimized design parameters of the FCM fuel assembly have been obtained.

By using the optimized FCM fuel assembly design data, the following analyses have been performed: neutron multiplication factor behavior, reactivity coefficients behavior, neutron spectrum effect, burnable poison effect, and behavior of fast neutron fluence.

From the results of the fuel assembly analysis, using the UN FCM fuel concept in current LWRs appears feasible. These preliminary findings need to be confirmed by the full-core performance analysis in the next fiscal year.

\section{Westinghouse Core}

The limited amount of analysis done on the $13 \times 13$ assembly replacement for the $17 \times 17 \mathrm{WH}$ fuel shows agreement on most issues with the analogous and more extensive work performed on the $12 \times 12$ FCM fuel replacement of the $16 \times 16$ System- 80 fuel assembly.

The required enrichment of the FCM fuel increase is dependent on the packing fraction value and the size of the TRISO particles. A simple invariance of the fissile fuel inventory is not sufficient and will yield shorter fuel cycles, because the burnup of the higher enrichment FCM fuel is faster.

The burnup simulation has shown that FCM fuel increases significantly the reactivity introduced by the fuel at BOL. However, the use of a lower amount of total heavy metal mass leads to faster depletion of the ${ }^{235} \mathrm{U}$ isotope, which is disadvantageous to the neutron economy by decreasing significantly the cycle length; therefore, the packing fraction or the enrichment needs to be increased more than necessary to match the initial fissile inventory. 
The MTC of the FCM fuel gives a less negative feedback during the first half of cycle compared to oxide fuel, which counteracts the loss of moderation capability after an increase in water temperature. Just as in the $12 \times 12$ analysis, the MTC positive behavior of the $13 \times 13$ FCM fuel assembly appears at the EOL only, which for the case analyzed is well beyond the point where the fuel would have to be extracted from the core because of lack of reactivity. The FTC behavior of the $13 \times 13$ FCM and the $17 \times 17$ reference cases is much more similar, indicating no major issues.

The CVR analysis shows a safer behavior for the $13 \times 13$ FCM fuel than the $17 \times 17$ reference. This could be important in the analysis of LOCA events. However, the full-core analysis is required to properly take into account core leakage effects in the analysis of the CVR.

The SERPENT code has proven to be a useful tool in modeling the geometry of the fuel assembly and modeling TRISO particles inside the desired matrix.

\subsection{REFERENCES}

Cho, J.Y., et al. 2007. DeCART v.1.2 User's Manual, KAERI/TR-3438/2007, KAERI (2007).

Grossbeck, M.L., et al. 2003. Development of Improved Burnable Poisons for Commercial Nuclear Power Reactors, Final Report on NERI Project Number 99-0074 (2003).

Jo, Chang Keun, Jongwha Chang, and F. Venneri. 2012. "A Neutronics Feasibility Study of Using FCM on a LWR," Transaction of ANS, Vol 106, Chicago, IL, June 24-28, 2012.

Joo, Han-Gyu, et al. 2004. "Methods and Performance of a Three-Dimensional Whole-Core Transport Code DeCART," PHYSOR 2004, Chicago, IL, USA, Apr. 25-29, 2004.

Kim, K.S. 2010. Benchmark Matrix for Verification and Validation of the KARMA Code, S06NX08-A-2TR-04, Rev.02, Jan. 21, 2010.

Shim, H.J., et al. 2012. "McCARD: Monte Carlo Code for Advanced Reactor Design and Analysis," Nucl. Eng. Tech., 44(2), 161-176 (2012). 


\section{CORE THERMAL-HYDRAULIC ASSESSMENT}

\subsection{TASK DESCRIPTION}

In order to demonstrate the thermal-hydraulic and safety compatibility of the FCM fuel in the existing LWR cores, it is necessary to start by performing the thermal-hydraulic analysis of the assembly for nominal conditions. Thermal and safety margins to DNB conditions were assessed and confirmed by determining the heat transport capability under normal, transient, and accident conditions. Fuel pressure drop analysis is used to evaluate the core flow distribution and fuel lift-up force. The assessment is sequentially performed at the subchannel, fuel assembly, and full-core levels.

In the first year, a subchannel analysis model based on the MATRA code was established for the thermal hydraulic scoping calculations on various FCM fuel geometries. A GUI (Graphical User Interface) utility program was developed for the convenient use and quality assurance of the subchannel analysis using the MATRA code. Fuel design selection criteria were established for the investigation of thermal hydraulic compatibility of the FCM fuel in the OPR-1000 core, based on the design practice for the reference reactor.

A systematic analysis was conducted for various fuel rod diameters, guide tube diameters, and spacer grids including IFM (intermediate flow mixer) grids. Pressure drop characteristics were evaluated for various bundle geometries to investigate the mechanical integrity of fuel assembly hold-down spring and the vibration of the fuel rod induced by crossflow. DNBR characteristics were investigated by employing appropriate critical heat flux (CHF) prediction models such as the CE-1 or W-3 correlations. The influence of IFM grid on DNBR was evaluated using an empirical correlation, which is applicable to various grid spacing conditions. Reference values for the bundle pressure drop and the minimum DNBR were calculated for the $16 \times 16$ reference fuel assembly under OPR-1000 operating conditions. These values were compared with those for the FCM fuel assemblies to satisfy the fuel selection criteria. At the end of the analysis, optimum geometries for $12 \times 12$ and $16 \times 16$ FCM fuel assemblies were suggested for the OPR-1000 core.

\subsection{ANALYSIS METHODS AND MODELS}

\subsubsection{MATRA}

A subchannel analysis method was employed for the investigation of thermal-hydraulic feasibility of the FCM LWR core. KAERI has developed the subchannel analysis code MATRA (Multi-channel Analyzer for steady state and Transients in Rods Array) in order to analyze the thermal-hydraulic characteristics in rod bundles for several kinds of nuclear reactors - the pressurized water reactor (PWR), liquid metalcooled reactor, as well as the gas-cooled reactor. The MATRA code has been systematically assessed for various bundle test data against a wide range of flow conditions such as the CHF data base for rod bundles.

The MATRA code adopts three conservation equations for the mixture of two-phase flow. The continuity, energy, and axial/lateral momentum equations for an arbitrary subchannel $i$ are expressed as follows: 
Continuity:

$$
A \frac{\partial \rho_{m}}{\partial t}+\frac{\partial \dot{m}}{\partial z}+\sum_{j} w_{i j}=0
$$

Energy:

$$
A \frac{\partial}{\partial t} \rho_{m} h_{m}+\frac{\partial}{\partial z}(\dot{m} h)+\sum_{j} w_{i j} h^{*}+\sum_{j} w_{i j}^{\prime}\left(h_{i}-h_{j}\right)=Q
$$

Axial momentum:

$$
\frac{\partial \dot{m}}{\partial t}+\frac{\partial}{\partial z}\left(\frac{\dot{m}^{2} v^{\prime}}{A}\right)+\sum_{j} w_{i j} u^{*}+f_{T} \sum_{j} w_{i j}^{\prime}\left(u_{i}-u_{j}\right)=-A \frac{\partial P}{\partial z}-F_{z}
$$

Lateral momentum:

$$
\frac{\partial w_{i j}}{\partial t}+\frac{\partial}{\partial z}\left(w_{i j} \bar{u}_{i}\right)+\frac{1}{l} \sum_{j} w_{i j} \bar{v}_{i}=\frac{s_{i j}}{l}\left(P_{i}-P_{j}\right)-F_{i j}
$$

The source terms in the above equations are expressed as

$$
\begin{aligned}
& Q=\frac{\partial}{\partial z}\left(k \frac{\partial T}{\partial z} A\right)-\sum_{j} \frac{k_{j} s_{i j}}{l}\left(T_{i}-T_{j}\right)+\sum_{n} \xi_{n}\left\langle q_{n}{ }_{n}\right\rangle \\
& F_{z}=\frac{1}{2}\left\{\frac{f \phi^{2}}{d_{h y} \rho_{m}}+\frac{K v^{\prime}}{\Delta x}\right\} \frac{|\dot{m}| \dot{m}}{A}+A \rho_{m} g \cos \theta \\
& F_{i j}=\frac{1}{2} K_{i j} \frac{w_{i j}\left|w_{i j}\right|}{\rho^{*} s_{i j} l}+s_{i j} \rho_{m, i} g \sin \theta
\end{aligned}
$$

The variables in the above equations are $A=$ flow area, $\rho_{m}=$ mixture density, $m=$ mass flow rate, $w_{i j}=$ cross flow from $i$ to $j$ channel, $h_{m}=$ mixture enthalpy, $h=$ flowing enthalpy, $w_{i j}^{\prime}=$ turbulent mixing flow rate, $v^{\prime}=$ effective specific volume considering phasic slip, $u=$ axial flow velocity, $f_{T}=$ turbulent momentum factor, $P$ = pressure, $l=$ centroid distance between subchannels, $s_{i j}=$ gap between subchannels $i$ and $j, k=$ thermal conductivity of fluid, $v=$ lateral velocity, $T=$ fluid temperature, $\xi=$ heated perimeter, $q^{\prime \prime}=$ heat flux,$f=$ friction loss coefficient, $\phi^{2}=$ two-phase friction multiplier, $K=$ form loss factor, $d_{h y}=$ hydraulic diameter, $g=$ gravity acceleration, $\theta=$ channel inclination angle from the vertical, and the superscript “*” means a donor cell property. 
The last term of the left-hand side of the conservation equations (4-1), (4-2) and (4-3) represents the net exchange of a mass, energy, and axial momentum due to a turbulent mixing between subchannel $i$ and its surrounding subchannels. By introducing a turbulent mixing parameter, $\beta$, which is defined as the ratio of the lateral fluctuating mass flux to the axial mass flux of the fluid in the subchannel, the turbulent mixing flow rate per unit length from subchannel $i$ to $j$ is expressed as

$$
w_{i j}^{\prime}=\beta \cdot s_{i j} \cdot G_{a v g}
$$

The turbulent mixing parameter is normally determined from a thermal mixing test in single-phase conditions. The two-phase mixing phenomenon is still not well understood and is formulated to be identical to the single-phase mixing model in the COBRA-IV-I code. The turbulent mixing model employed in the COBRA-IV-I code is based on the equal mass exchange (EM) concept. In the EM model, it is assumed that the fluctuating mass flow rates between the interacting subchannels are identical. Thus there is no net mass transfer due to turbulent mixing, and a diversion crossflow is the only mechanism transferring mass between the subchannels.

To obtain the numerical solutions for the above-mentioned equations, an implicit solution scheme was employed in the MATRA code. A fully implicit backward differencing scheme is used for the discretization of the governing equations, while an upwind differencing is exclusively used for the convective terms. The major variables, such as the axial flow, crossflow, enthalpy, pressure, and density, are calculated simultaneously for all the subchannels through an external iterative sweep of the computational mesh from the bottom of the channel. A solution is found when the maximum changes in crossflow, axial flow, and enthalpy satisfy the convergence criteria simultaneously. The boundary conditions imposed on this problem are (1) specified inlet flow and enthalpy distributions and (2) zero inlet crossflow and uniform pressure at the exit of the bundle.

\subsubsection{Subchannel Analysis Modeling}

The influence of various fuel assembly geometry parameters, such as P/D (rod pitch-to-diameter ratio), type of rod array, and rod diameter, is properly reflected in the MATRA code with ad hoc thermal hydraulic models. Table 11 shows the constitutive models for the thermal-hydraulics analysis of FCM assemblies.

For the feasibility analysis of the FCM LWR fuel, a quarter symmetry and full-scale FCM fuel assembly models were adopted, referencing the OPR-1000 core conditions. The cross-sectional views of a reference $16 \times 16$ fuel assembly and a $12 \times 12$ candidate assembly for the optimized FCM fuel core are shown in Figure 30 .

In order to suggest a desirable geometry in thermal-hydraulic viewpoints, several design criteria for the optimization were selected, especially for the assembly pressure drop and the minimum DNBR. Since the feasibility analysis requires a series of MATRA calculations with various fuel assembly geometries, a GUI utility for the MATRA code was developed to automate the procedure of MATRA calculation. The utility program generates input data and provides automatic output processing with MATRA. The GUI utility was built in order to generate the MATRA input data of FCM fuel assembly and to process the MATRA output using the $\mathrm{C}++$.net framework, as shown in Figure 31. Rod configuration is available for the quarter symmetry and full assembly models for $12 \times 12$ and $16 \times 16$ grids. The utility is supplied with a look-for function that searches for optimum design values of the control parameter that satisfies the design criteria for pressure drop and minimum departure from nucleate boiling ratio (MDNBR). Control parameters of the function are the diameter of heated rod (fuel) and unheated rod (guide tube), rod pitch-to-diameter ratio, and fuel assembly pitch. The utility can provide a text-mode 
result as well as graphical display to help understand the arrangement of the FCM fuel assembly under consideration. In addition, this utility has the ability to post-process the calculated result and show the contour of variables such as enthalpy, quality, mass flux, and so on. Finally, the repeated execution of MATRA calculations is supported. In the Hydraulic View, variations in pressure drop and MDNBR as a function of important parameters of the FCM fuel assembly design are displayed, for various P/D ratios. The major features of the GUI utility program are illustrated in Figure 32.

Table 11. MATRA models for scoping analysis of FCM fuel assemblies

\begin{tabular}{|c|c|}
\hline Parameters & Values \\
\hline Flow models & \\
\hline - Field equations & Homogeneous mixture \\
\hline - Subcooled boiling void fraction & Saha-Zuber model \\
\hline - Bulk boiling void fraction & Chexal-Lellouche model \\
\hline - Two-phase friction multiplier & Homogeneous model \\
\hline Subchannel interaction models & \\
\hline - Crossflow resistance factor & 0.5 \\
\hline - Turbulent mixing parameter for single-phase & 0.038 \\
\hline - Two-phase turbulent mixing model & Equal-mass-exchange model \\
\hline Empirical TH models & \\
\hline - Bundle friction factor & $0.184 \mathrm{Re}^{-0.2}$ \\
\hline - Spacer grid loss factor & $0.946(\mathrm{MV}), 0.494(\mathrm{HTP})$ \\
\hline - Critical heat flux correlations & CE-1, W-3, KRB-1 \\
\hline Numerical parameters & \\
\hline - Number of axial nodes in active length & 50 \\
\hline - Solution scheme & Marching scheme with SOR \\
\hline - Boundary conditions & Inlet flow/Exit pressure \\
\hline - Convergence criteria for & \\
\hline axial flow & 1.E-2 \\
\hline crossflow (internal/external) & 1.E-3 / 1.E-1 \\
\hline
\end{tabular}




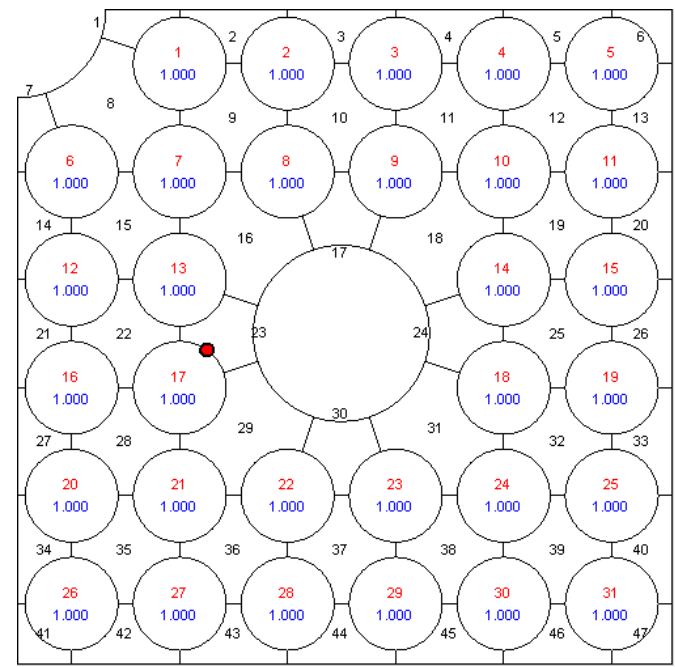

$\underline{12 \times 12 \text { quarter symmetry model }}$

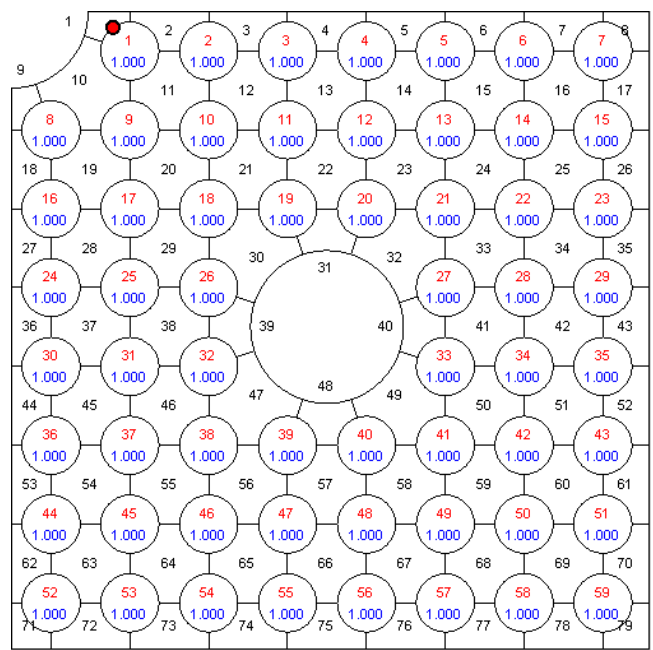

$\underline{16 \times 16 \text { quarter symmetry model }}$ (Red dot represents the MDNBR location)

Fig. 30. Cross-sectional view of $12 \times 12$ and $16 \times 16$ fuel assemblies.

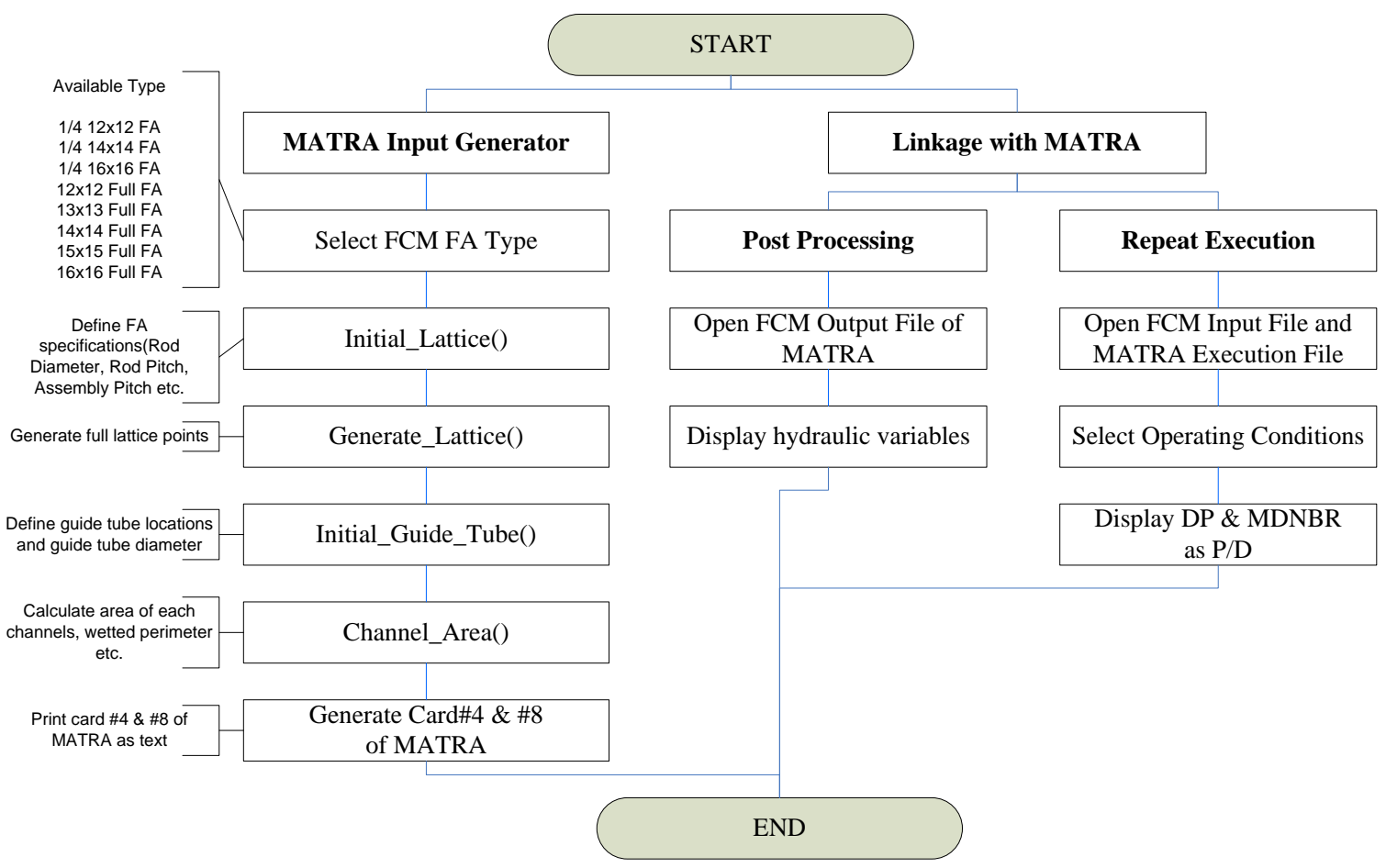

Fig. 31. Flow chart of MATRA input generator for FCM fuel design. 


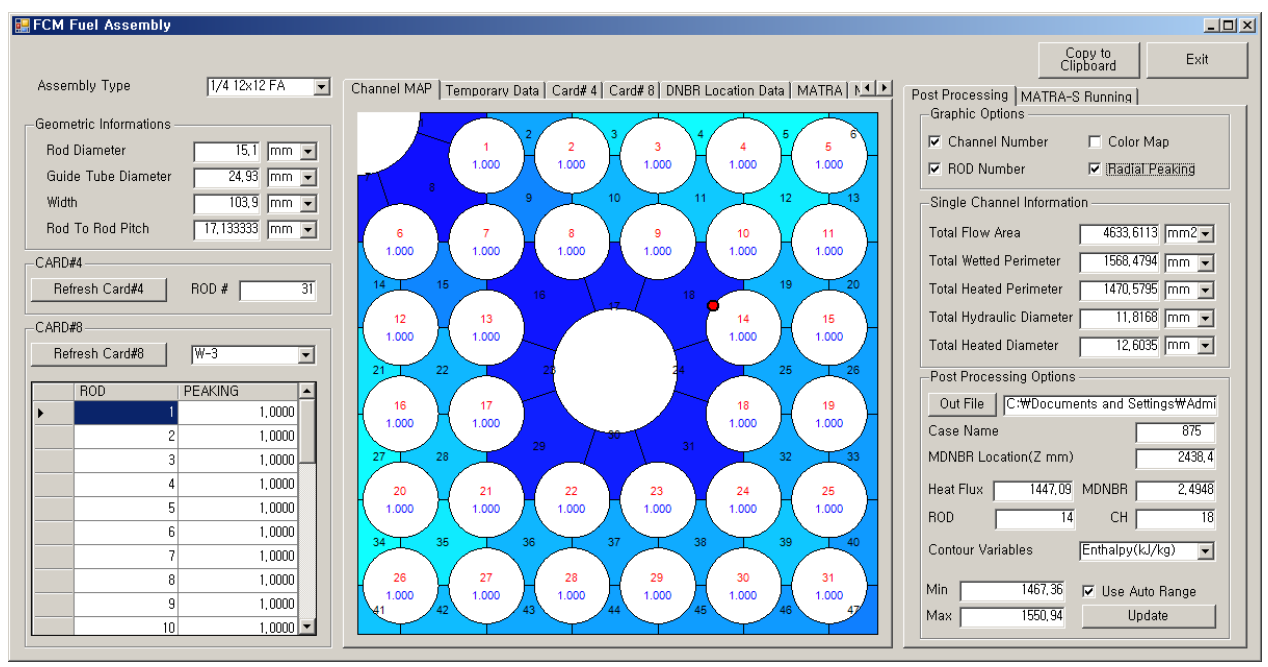

a) Post-Processing mode

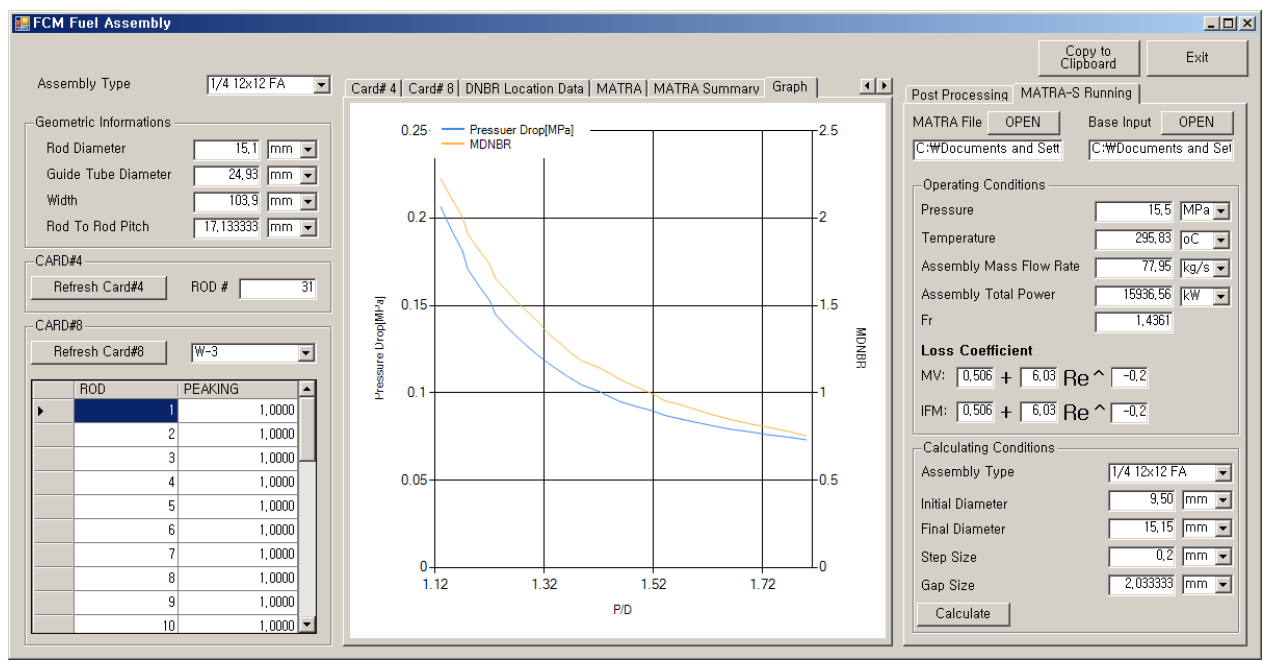

b) Repeat-Execution mode

Fig. 32. Illustrations of MATRA GUI utility for FCM fuel.

\subsection{PRELIMINARY SCOPING ANALYSIS FOR OPR-1000 CORE}

In order to investigate an acceptable geometry for FCM fuel in the OPR-1000 core, the selection criteria of FCM fuel design with respect to thermal and hydraulic performance are determined as described in Table 12. The assembly pitch and the position of the guide tubes are the fixed boundary conditions for the selection of the FCM fuel geometry.

The fuel rod outer diameter affects the amount of uranium loading and the fuel-to-moderator ratio, which are important in order to evaluate core management parameters such as cycle length and reactivity coefficients. From the thermal-hydraulic viewpoint, the fuel rod outer diameter is related to the heat transfer area and the flow area, which both directly affect the bundle pressure drop and thermal margin characteristics. 
The rod-to-rod gap size is related to the spacer grid design. The fuel assembly design, which consists of fuel rods, guide tubes, and spacer grids and other components, needs to be examined with regard to its thermal-hydraulic compatibility with the existing 16×16 reference OPR-1000 fuel assemblies, particularly in the transition cores.

A pressure drop limit criterion for the fuel assembly was selected to ensure hydraulic compatibility with respect to up-lift force and maximum crossflow. Securing a thermal margin of $15 \%$ is the target value for the homogeneous core of FCM fuel assemblies in OPR-1000.

Table 12. Thermal-hydraulic selection criteria for FCM fuel

\begin{tabular}{|c|c|c|c|}
\hline & Parameter & Criteria & Note \\
\hline \multirow{2}{*}{ Fuel Rod } & Rod outer diameter & $10.75-15.9 \mathrm{~mm}$ & \multirow{2}{*}{$\begin{array}{l}\text { Core management \& spacer } \\
\text { grid design }\end{array}$} \\
\hline & Gap of rod to rod & Greater than $2.0 \mathrm{~mm}$ & \\
\hline \multirow{6}{*}{$\begin{array}{c}\text { Fuel } \\
\text { Assembly } \\
\text { (FA) }\end{array}$} & FA array & $12 \times 12-16 \times 16$ & Core configuration \\
\hline & Pressure drop & Greater than existing FA & \multirow[b]{2}{*}{ Transition core compatibility } \\
\hline & FA lift force & $\begin{array}{c}\text { Less than } 20 \% \text { increase in } \\
\text { existing FA }\end{array}$ & \\
\hline & Maximum crossflow & Less than $1 \mathrm{~m} / \mathrm{sec}$ & Prevent rod vibration \\
\hline & Spacer type & Grid-type, fin-type, etc. & $\begin{array}{c}\text { Transition core compatibility } \\
\text { Thermal margin }\end{array}$ \\
\hline & DNBR & $\begin{array}{c}\text { Not deteriorate DNBR for } \\
\text { existing FA }\end{array}$ & Transition core compatibility \\
\hline Core & Thermal margin & $15 \%$ & Target for FCM initial core \\
\hline
\end{tabular}

Systematic calculations have been conducted for the various criteria by changing the rod outer diameter and guide tube diameter, as summarized in Table 13.

Table 13. Calculation conditions of fuel assembly

\begin{tabular}{|l|c|c|c|c|}
\hline \multirow{2}{*}{\multicolumn{1}{c|}{ Parameters }} & \multirow{2}{*}{ Unit } & \multicolumn{2}{|c|}{ FCM fuel assembly } & Reference fuel assembly \\
\cline { 3 - 5 } & & $\mathbf{1 2} \times \mathbf{1 2}$ & $\mathbf{1 6} \times \mathbf{1 6}$ & $\mathbf{1 6 \times 1 6}$ \\
\hline Rod diameter & $\mathrm{mm}$ & $12.67-16.34$ & $9.5-12.29$ & 9.5 \\
\hline Guide tube diameter & $\mathrm{mm}$ & $24.93-35.00$ & 24.93 & 24.93 \\
\hline P/D ratio & - & $1.05-1.35$ & $1.05-1.35$ & 12.85 \\
\hline Wetted perimeter & $\mathrm{mm}$ & $5328-6757$ & $7434-9503$ & 7434 \\
\hline Heated perimeter & $\mathrm{mm}$ & $4936-6365$ & $7043-9112$ & 7043 \\
\hline Hydraulic diameter & $\mathrm{mm}$ & $8.72-18.85$ & $5.02-12.92$ & 12.92 \\
\hline Flow area & $\mathrm{m}^{2}$ & $0.1474-0.2455$ & $0.1276-0.2402$ & 0.2402 \\
\hline Grid type & - & MV/HTP & MV/HTP & MV \\
\hline FA power & $\mathrm{MW}$ & 15.94 & 15.94 & 15.94 \\
\hline Average heat flux & $\mathrm{kW} / \mathrm{m}^{2}$ & $944-1217$ & $659-853$ & 853 \\
\hline FA flow rate & $\mathrm{kg} / \mathrm{sec}$ & 80.75 & 80.75 & 80.75 \\
\hline Average mass flux & $\mathrm{kg} / \mathrm{m}^{2}-\mathrm{sec}$ & $3215-5478$ & $3362-6325$ & 3362 \\
\hline Radial power distribution & - & uniform & uniform & uniform \\
\hline Axial power shape & - & cosine & cosine & cosine \\
\hline
\end{tabular}




\subsubsection{Pressure Drop}

The pressure drop in the fuel assembly was calculated by

$$
\Delta P=\left(f \frac{L}{d_{h y}}+K_{g r i d}\right) \times \frac{G^{2}}{2 \rho}
$$

The friction loss coefficient was determined by

$$
f=0.184 \times \operatorname{Re}^{-0.2}
$$

The pressure drop characteristics for various designs of FCM fuel assemblies have been evaluated in the calculation conditions provided in Table 13. The important parameters affecting the pressure drop of fuel assembly are hydraulic diameter, mass velocity, and grid loss coefficient.

The pressure drop in the fuel assembly for various rod arrays from $12 \times 12$ to $16 \times 16$ is calculated with various rod diameters, as shown in Figure 33. As the number of rods increases with a constant flow rate at the fuel assembly inlet and constant grid loss coefficient, the pressure drop increases mainly due to the decrease of hydraulic diameter and the increase of mass velocity. The upper and lower limits of rod diameter may be bounded from the considerations of fuel-to-moderator ratio and required minimum gap size. If rod diameter is decreased while keeping constant values of flow rate, fuel assembly power, number of rods, and rod pitch (i.e., the P/D increases), the pressure drop will decrease due to the increased hydraulic diameter and decreased mass flux.

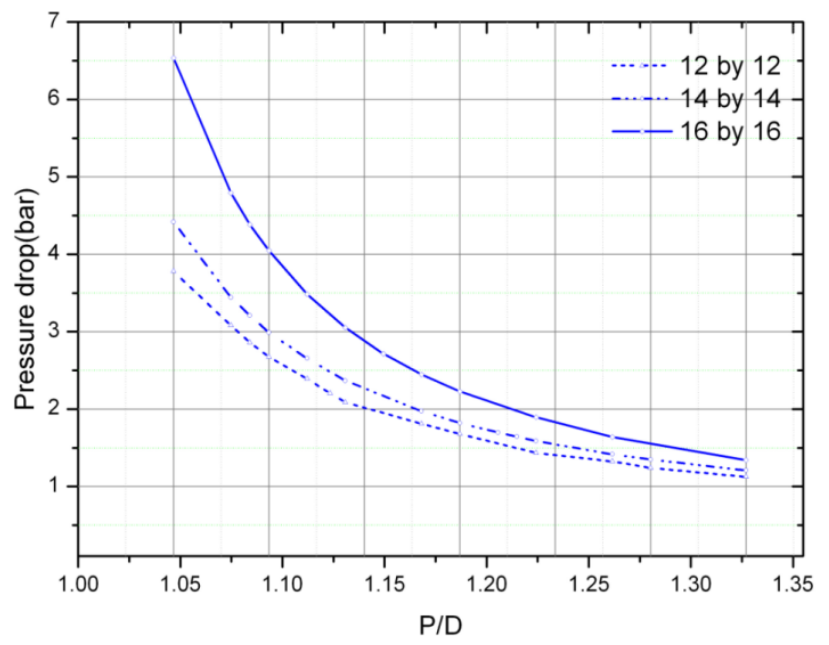

Fig. 33. Pressure drop variation for various rod diameters at different rod arrays.

Since the $16 \times 16$ fuel assembly for OPR-1000 core includes large guide tubes for control rods insertion and in-core instrumentation, the subchannel hydraulic diameter near the guide tube is usually large compared to that of the matrix subchannel, resulting in a mal-distribution of flow in the fuel assembly. Since the heterogeneity of flow distribution inside the fuel assembly may cause some decrease of thermal margin, it is desirable to achieve a more uniform flow distribution by adjusting the guide tube diameter. The increase of guide tube diameter should increase the fuel assembly pressure drop due to the decrease of hydraulic diameter. 
The influence of spacer grids on the pressure drop of fuel assembly was evaluated with two different types of the spacer grids: the Mixing Vane (MV) grid and the High Thermal Performance (HTP) grid. It is reported that the loss coefficient of the HTP grid is remarkably (approximately 50\%) lower than that of the MV grid, while the thermal performances for both grids are similar. In this scoping calculations we assumed that the DNBR performance of HTP grid and MV grid are the same (i.e., the same CHF correlation was applied to both to calculate DNBR). The pressure drop of FCM fuel assembly with HTP grids is estimated to be about $45 \%$ less than that with MV grids.

\subsubsection{DNBR}

The main thermal design requirement, as presented in Table 12, is that in order to ensure the validity of the safety analysis results for the reference core, the FCM fuel assembly should not decrease the thermal margin of the existing fuel assemblies. In addition, in order to preserve the thermal power capability of the reactor, the DNBR of the FCM fuel needs to be similar to or larger than the DNBR of the reference assembly.

The DNBR was calculated at fixed conditions of the bundle inlet mass flow rate and the bundle power. The DNBR for the reference $16 \times 16$ fuel assembly was calculated by employing the CE- $1 \mathrm{CHF}$ correlation. However, for the $12 \times 12$ fuel assembly, the CE- 1 correlation produced unrealistic DNBR values since the geometry parameters of the subchannels are beyond the applicable ranges of the CE-1 correlation. Therefore, a rod bundle $\mathrm{CHF}$ correlation with a wide applicable range of channel diameter, the W-3 CHF correlation, was selected for the calculation of DNBR for various geometries. A CHF correlation, the KRB-1 correlation, which includes grid spacing as a correlation parameter, was employed to evaluate the influence of the IFM grid on DNBR. The functional forms of the CE-1, W-3, and KRB-1 correlations are as follows.

CE-1 correlation:

$$
q_{C E 1}^{\prime \prime}=\frac{a_{1}\left(d_{h e} / d_{h e, \text { matrix }}\right)^{a_{2}}\left[\left(a_{3}+a_{4} P\right) G^{\left(a_{5}+a_{6} P\right)}-G h_{f g} \chi_{c}\right]}{G^{\left(a_{7} P+a_{8} G\right)}}
$$

W-3 correlation:

$$
\begin{aligned}
& q^{\prime \prime}{ }_{W 3}=\left\{b_{1}-b_{2} P+\left(b_{3}-b_{4} P\right) \mathrm{e}^{\left(b_{5}-b_{6} P\right) x}\right\} \times\left\{\left(b_{7}-b_{8} x+b_{9} x|x|\right) G+b_{10}\right\} \\
& \times\left(b_{11}-b_{12} x\right) \times\left\{b_{13}+b_{14} \mathrm{e}^{-b_{15} d_{h y}}\right\} \times\left\{b_{16}+b_{17}\left(h_{f}-h_{\text {in }}\right)\right\} \times\left\{1+b_{18} G\left(\frac{\beta}{0.019}\right)^{0.35}\right\}
\end{aligned}
$$

KRB-1 correlation:

$$
\begin{aligned}
q^{\prime \prime}{ }_{K R B 1}= & \left\{c_{10}+c_{1} L+c_{2} P+c_{3} d_{h e}+c_{4} d_{h y}+c_{5} \tanh \left[c_{11}\left(d_{g}+g_{s p}\right)+c_{12}\right]\right\} \\
& -\left\{c_{6} P+c_{7} P L+c_{8} L+c_{9} P^{2}\right\} \cdot G \cdot \chi_{c}+c_{13} \cdot G
\end{aligned}
$$

If the rod diameter is decreased while maintaining a fixed the number of rods in the assembly, the DNBR decreases (i.e., the thermal margin decreases) due to the increase of average heat flux and the decrease of the average mass flux. The influence of the guide tube diameter was also investigated with a fixed rod-to- 
rod gap size of $2 \mathrm{~mm}$, which is approximately the lower limit value for the mechanical design of spacer grids. As the guide tube diameter increases, the thermal margin also increases due to the increase of the average mass flux and to the positive effects of subchannel flow distribution to the minimum DNBR (i.e., the subchannel flow distribution becomes more uniform).

The influence of spacers with IFM grids on the thermal performance was evaluated using the KRB-1 CHF correlation with the MATRA code. The axial distributions of enthalpy and mass flux at the hot subchannel for $12 \times 12$ and $16 \times 16$ fuel assemblies are compared in Figure 34 . The minimum DNBR occurs near the middle of the heated region due to the cosine axial power shape. The IFM grid increased the DNBR and the pressure drop. The DNBR with IFM grid was increased by approximately $8 \%$, in comparison with the reference DNBR value, while the pressure drop increased by about $5 \%$. Since the increase in pressure drop may reduce the assembly flow rate in the transition core, the net DNBR gain of IFM grid may be lower than $8 \%$. In this scoping analysis, the DNBR gain by an IFM grid was conservatively approximated as $3 \%$.

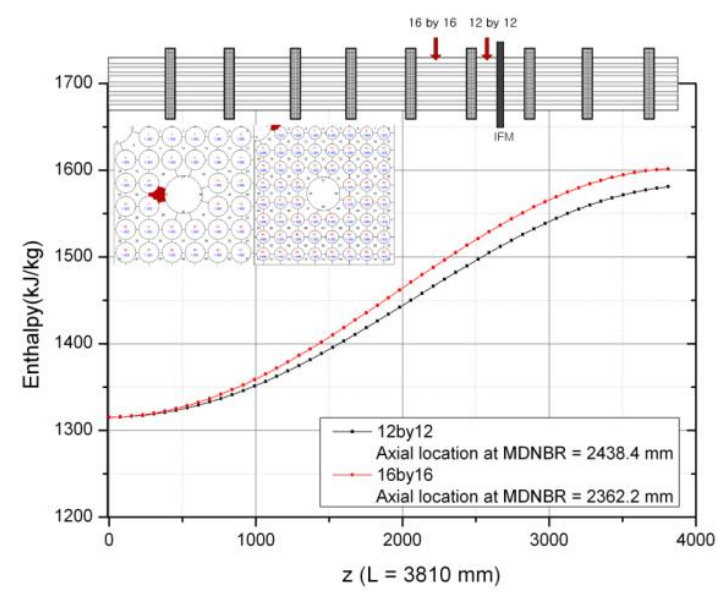

Hot channel enthalpy

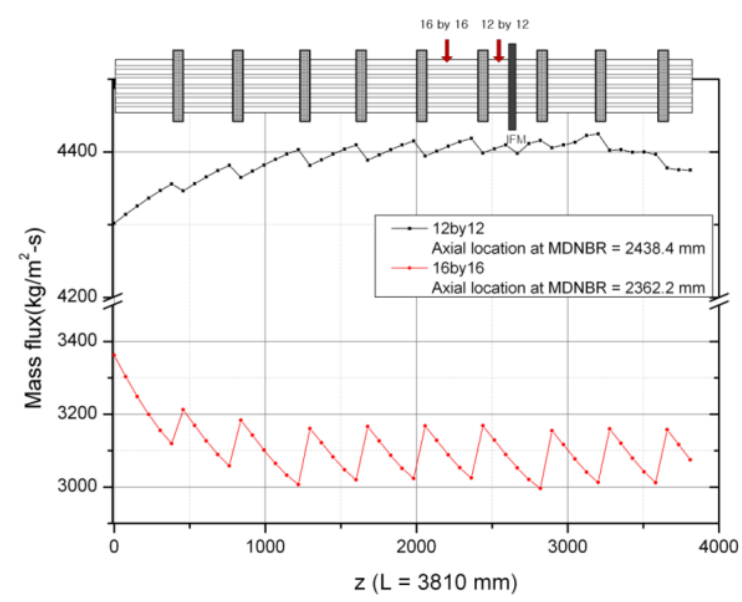

Hot channel mass flux

Fig. 34. Axial distributions of hot channel enthalpy and mass flux for $12 \times 12$ and $16 \times 16$ candidate FCM fuel assembly configurations.

\subsubsection{Fuel Assembly Design Optimization}

The reference values for the assembly pressure drop (DP) and MDNBR at the nominal operating condition of OPR-1000 were calculated as 1.327 bar and 2.039 (DP and DNBR by CE-1 correlation) or 2.330 (DNBR by W-3 correlation). The acceptable range of the pressure drop for FCM fuel was therefore selected to be between 1.327 and 1.592 bar, considering a 20\% margin. The minimum DNBR for the FCM fuel should be greater than the reference value.

Figures 35 and 36 show the optimum design points on the ratio of rod pitch to diameter. In the figures, the red-hatched and blue-hatched areas denote the acceptable regions of thermal design criteria and hydraulic design criteria, respectively. The optimum fuel assembly geometry should satisfy both design criteria simultaneously. As a result of the scoping analysis on the thermal-hydraulic compatibilities, the acceptable range of rod diameter for the $12 \times 12$ FCM fuel assembly with HTP grids was found to be between $14.60 \mathrm{~mm}$ and $15.05 \mathrm{~mm}$. For the fuel assembly with MV grids, the acceptable range was found to be between $12.94 \mathrm{~mm}$ and $14.00 \mathrm{~mm}$. For the $16 \times 16$ fuel assembly, the acceptable range of rod diameter with MV grids was found to be between $9.5 \mathrm{~mm}$ and $10.04 \mathrm{~mm}$. 
Within the acceptable ranges of fuel geometries, an optimum design for the $12 \times 12$ FCM fuel assembly was selected, with a rod diameter of $14.80 \mathrm{~mm}$ spaced by HTP grids with a single IFM grid as shown in Figure 37. For the $16 \times 16$ FCM fuel assembly, the rod diameter of $10.0 \mathrm{~mm}$ with MV grids (the same as the reference fuel assembly) was recommended. The main design features for the optimum FCM fuel assemblies are summarized in Table 14.

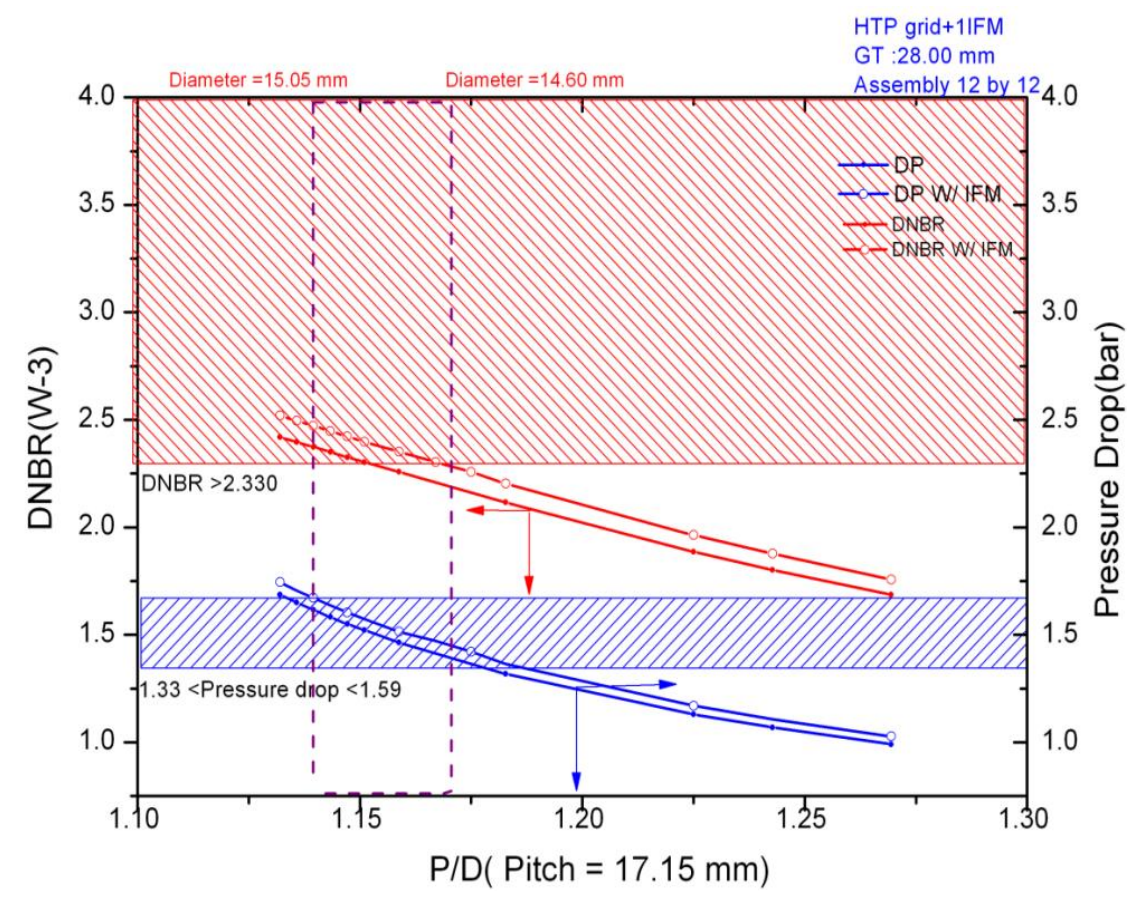

Fig. 35. DNBR and pressure drop on the optimized $12 \times 12$ FCM fuel.

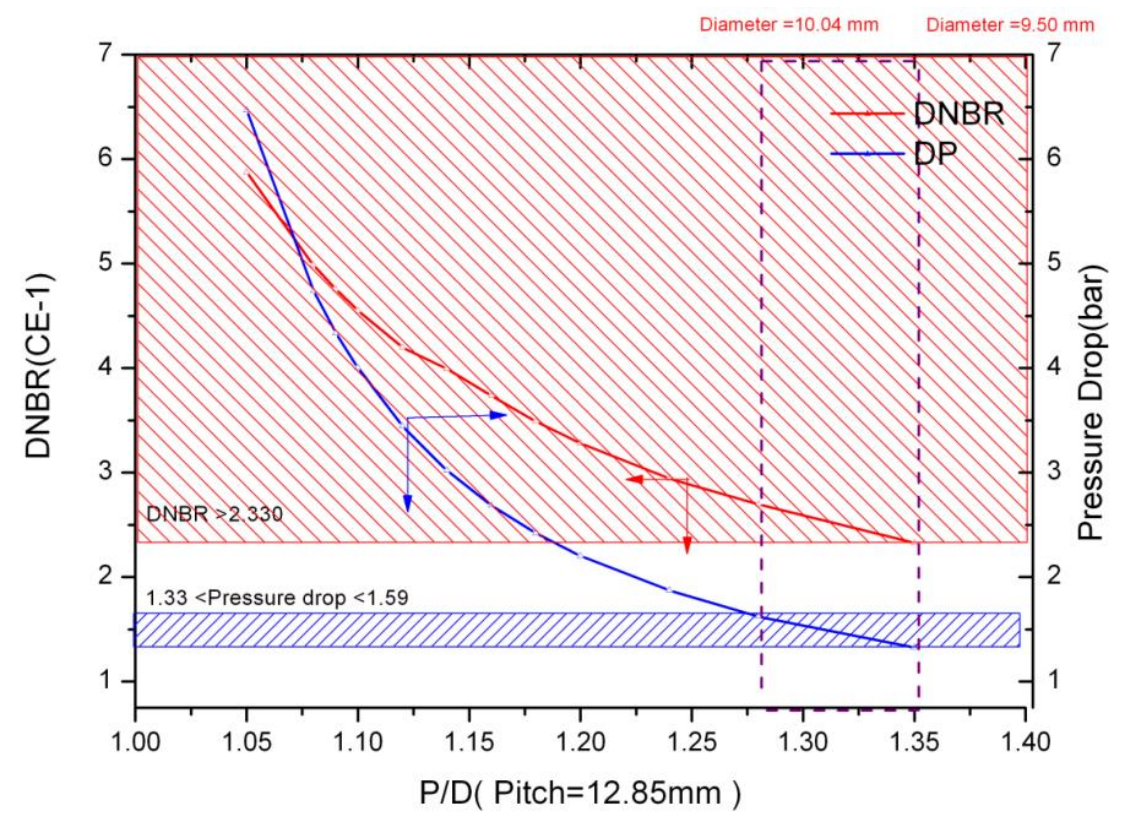

Fig. 36. DNBR and pressure drop on the optimized 16×16 FCM fuel. 


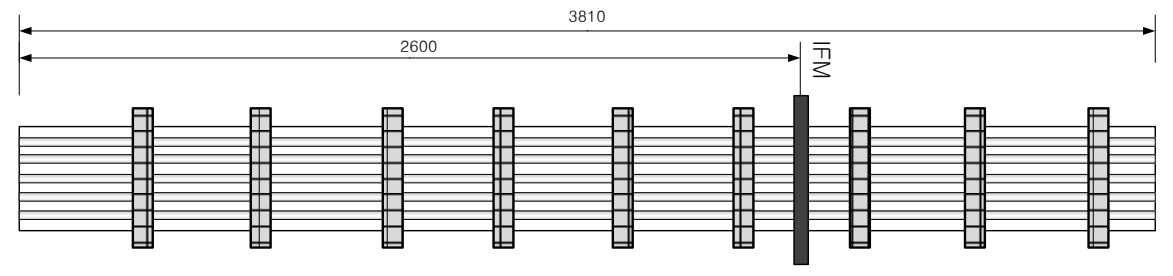

Fig. 37. Axial geometry of optimum $12 \times 12$ FCM fuel assembly.

Table 14. Optimum fuel assembly design specification

\begin{tabular}{|c|c|c|c|c|}
\hline \multirow{2}{*}{\multicolumn{2}{|c|}{ Parameters }} & \multicolumn{2}{|c|}{ Optimized FCM } & \multirow{2}{*}{$\begin{array}{c}\text { OPR-1000 } \\
16 \times 16\end{array}$} \\
\hline & & $12 \times 12$ & $16 \times 16$ & \\
\hline \multicolumn{2}{|c|}{ Guide Tube diameter ( $\mathrm{mm})$} & 28.0 & 24.93 & 24.93 \\
\hline \multicolumn{2}{|c|}{ Rod diameter (mm) } & 14.8 & 10.0 & 9.5 \\
\hline \multicolumn{2}{|c|}{ Pitch-to-diameter ratio, P/D } & 1.159 & 1.29 & 1.36 \\
\hline \multicolumn{2}{|c|}{ Gap size $(\mathrm{mm})$} & 2.35 & 3.35 & 3.4 \\
\hline \multicolumn{2}{|c|}{ Average heat flux $\left(\mathrm{kW} / \mathrm{m}^{2}\right)$} & 1042 & 810 & 853 \\
\hline \multicolumn{2}{|c|}{ Average mass flux $\left(\mathrm{kg} / \mathrm{m}^{2}-\mathrm{s}\right)$} & 4302 & 3636 & 3362 \\
\hline \multicolumn{2}{|c|}{ Hydraulic dia. $(\mathrm{mm})$} & 12.10 & 11.38 & 12.92 \\
\hline \multicolumn{2}{|c|}{ Spacer grid } & HTP grid (1-IFM) & PLUS7 & PLUS7 \\
\hline \multicolumn{2}{|c|}{ Pressure drop (bar) } & 1.515 & 1.569 & 1.327 \\
\hline \multirow{2}{*}{ DNBR } & W-3 correlation & 2.351 & - & 2.330 \\
\hline & CE-1 correlation & - & 2.245 & 2.039 \\
\hline
\end{tabular}

\subsection{PLANNED ACTIVITIES FOR NEXT YEAR}

\subsubsection{Scoping Analysis for OPR-1000}

It is planned to refine the DNBR and pressure drop analysis models for the FCM fuel with various spacers. Thermal-hydraulic optimization will be carried out accounting for the refined design features. In addition, thermal-hydraulic analysis will be conducted for the homogeneous and/or transition core of FCM-fuel-loaded OPR-1000.

\subsubsection{Scoping Analysis for WH Plants}

It is planned to perform the DNBR and pressure drop analysis for the FCM fuel.

\subsection{SUMMARY}

Thermal-hydraulic analysis models for the FCM fuel assembly have been established using the subchannel analysis code MATRA. Pertinent models for preliminary evaluation of the assembly pressure drop and DNBR are employed in the MATRA code. A GUI utility program is developed for a parametric study on the geometry of fuel assembly.

From the preliminary scoping analysis, optimum geometries of the $12 \times 12$ and $16 \times 16$ FCM fuel assemblies are proposed meeting the design selection criteria for pressure drop and DNBR of the OPR-1000 core. 


\subsection{REFERENCES}

CE, C-E critical heat flux: critical heat flux condition for CE fuel assemblies with standard spacer grids, Part 1 uniform axial power distribution, CENPD-162, 1976.

Cheng, X. and D.H. Hwang. 2005. "Subchannel analysis of LBE-cooled fuel assemblies of accelerator driven systems," Proceedings of GLOBAL 2005, paper 115, Tsukuba, Japan, 2005.

Han, K.H., K.W. Seo, D.H. Hwang, and S.H. Chang. 2006. "Development of a thermal hydraulic analysis code for gas-cooled reactors with annular fuels," Nucl. Eng. Design, 236, pp.164, (2006).

Hwang, D.H., K.W. Seo, and H. Kwon. 2008. "Assessment of critical heat flux data base for rod bundles,” Proceeding of KNS Spring Meeting, Kyeongju, Korea, 2008.

Hwang, D.H., S.Y. Chun, K.K. Kim, and C.C. Lee. 2008. "Mass velocity and cold-wall effects on critical heat flux in an advanced light water reactor," Nucl. Eng. Design, 237, pp.369, (2007).

Hwang, D.H., Y.J. Yoo, J.R. Park, and Y.J. Kim. 1995. "Evaluation of the thermal margin in a KOFAloaded core by a multichannel analysis methodology," Nucl. Eng. Tech. 27, pp.518, (1995).

Tong, L.S. 1972. Boiling crisis and critical heat flux, TID-25887, Westinghouse, 1972.

Yoo, Y.J., D.H. Hwang, and D.S. Sohn. 1999. "Development of A Subchannel Analysis Code MATRA Applicable to PWRs and ALWRs," J. Korean Nuclear Society, 31, 314 (1999). 


\section{SAFETY ASSESSMENT}

\subsection{TASK DESCRIPTION}

The safety of FCM fueled cores is assessed for three DBA scenarios: LOFAs for DNB margin, LOCAs for PCT margin and rod ejection accident (REA) for pellet cladding mechanical interaction (PCMI) margin. In addition, the accident analysis for BDBA scenarios such as SBO and LBLOCA without safety injection, including multiple safety system failures, is carried out to quantify the accident-tolerant envelope of the FCM fuel.

\subsection{ANALYTIC METHODS AND MODELS}

\subsubsection{MARS}

The MARS (Multi-dimensional Analysis of Reactor Safety) code was used for the realistic accident analysis of FCM fueled cores. The backbones of MARS are the RELAP5/MOD3.2.1.2 and COBRA-TF codes of the US NRC. The RELAP5 code is a versatile and robust systems analysis code based on a onedimensional, two-fluid model for two-phase flow, whereas the COBRA-TF code is based on a threedimensional, two-fluid, three-field model. The two codes were consolidated into a single code by integrating the hydrodynamic solution schemes, and unifying various thermal-hydraulic models, equations of state (EOS), and input/output (I/O) features. The source of the codes was fully restructured using the modular data structure and new dynamic memory allocation scheme of FORTRAN 90. In addition, a generic multi-dimensional fluid model was developed and implemented to the RELAP5 system analysis module in order to overcome some limitations of the COBRA-TF 3D vessel module. MARS runs on the Windows platform, and it is currently used as a popular multi-dimensional thermalhydraulic tool for the analyses of reactor transients, experimental facility simulations, and other safety analysis purposes. MARS can also be connected, by dynamic link libraries (DLLs), to other codes such as the 3D kinetics code and the containment analysis codes. A new capability for GEN-IV reactor analysis was also implemented by adding gas property tables and new heat transfer correlations. The last version of this series is MARS3.1, which was released in 2005 (KAERI, 2006).

MARS3.1 can be utilized for the simulation of DBA transients such as loss of coolant, anticipated transients without scram (ATWS), and operational transients such as loss of flow, loss of feed-water, loss of off-site power, and turbine trip.

\subsubsection{Reference Core and System Modeling}

The FCM fuel is designed to be used in the conventional PWR, with the System 80+ OPR-1000 reactor selected as the reference plant. Figure 38 shows the MARS nodalization of OPR-1000 for safety analysis. The core consists of two channels, the hot core channel and the average core channel. The hot core channel includes "hottest fuel" and "hot fuel". "Hottest fuel" models a single hottest fuel rod, and "hot fuel" rod models the rods in the hottest assembly. The other rods are modeled as average rod in the average core channel.

The power distribution for the FCM-fueled core is calculated using OPR-1000 assembly and radial intraassembly peaking factors. The axial power distribution is assumed to be cosine shaped. The calculated power distribution is shown as Table 15 .

The FCM fuel pellet is composed by TRISO particles and SiC matrix, and they are mixed up nonuniformly. The inner structure of the FCM fuel however is not considered by MARS code, and the homogenized thermal properties are calculated from the mass fraction of each component. Among the 
components, the thermal conductivity of $\mathrm{SiC}$ has significant variability according to the manufacturing process and irradiation history. The thermal conductivity of the $\mathrm{SiC}$ coating layer is calculated from the thermal conductivity of unirradiated chemical-vapor-deposited (CVD) $\mathrm{SiC}$ and the thermal resistivity introduced by defects from fast neutron dose. Neutron irradiation in fact produces thermal defects in the molecular structure and causes degradation of the thermal conductivity. The thermal conductivity during irradiation is calculated as follows (Snead, 2007):

$\mathrm{K}=\left(1 / \mathrm{K}_{\text {Non-Irradiated }}+\text { Thermal Defect Resistance }\right)^{-1}$.

The thermal conductivity of the $\mathrm{SiC}$ matrix is calculated from an averaged ratio between CVD $\mathrm{SiC}$ and monolithic NITE SiC conductivity (Snead, 2012). This value is also used for the thermal conductivity of $\mathrm{SiC}$ cladding. From the calculations, thermal conductivity and volumetric heat capacity for FCM fuels are generated.

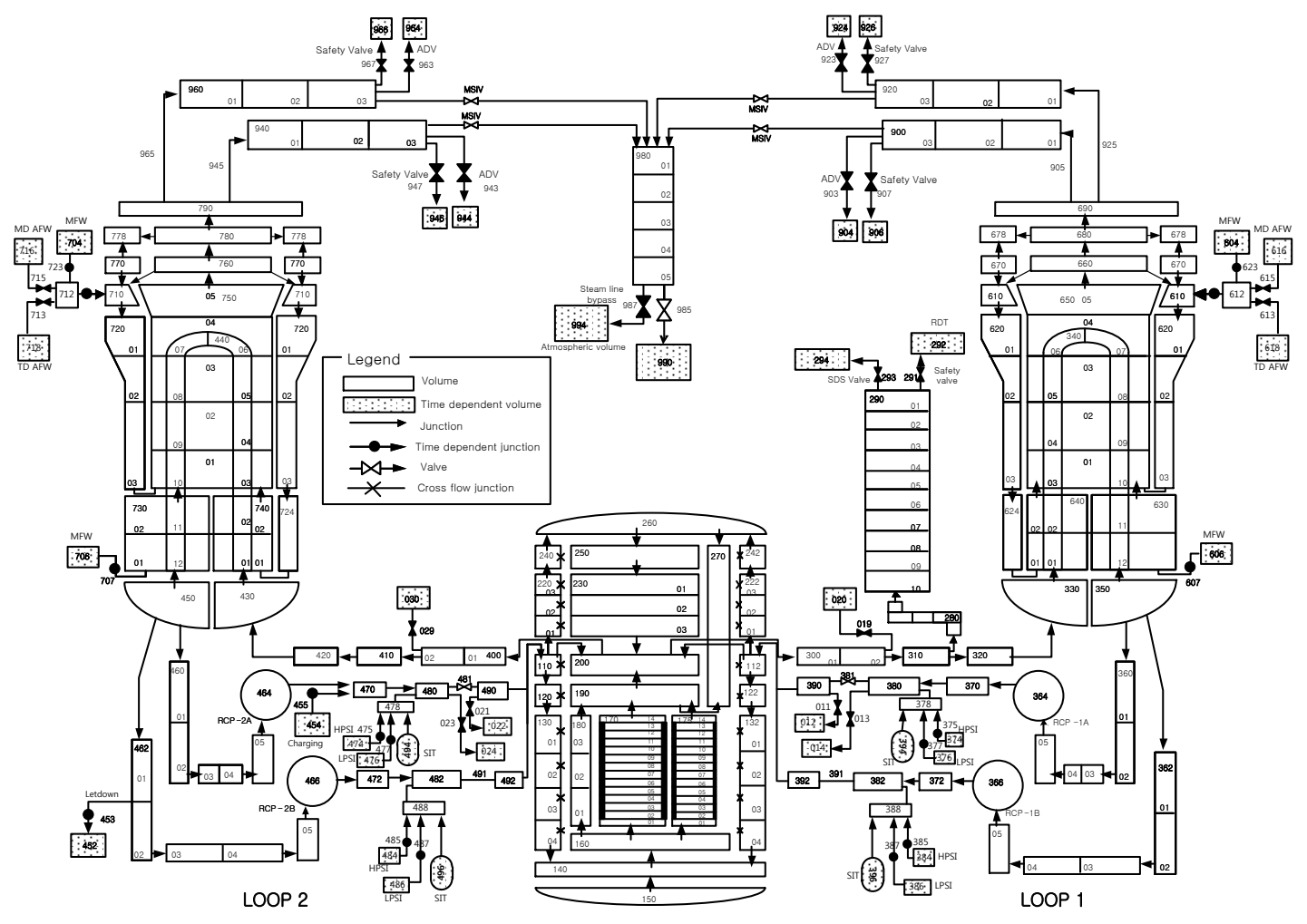

Fig. 38. Nodalization of OPR-1000. 
Table 15. Power distribution with fuel arrangement

\begin{tabular}{|c|c|c|c|c|c|c|}
\hline \multirow{2}{*}{$\begin{array}{c}\text { Core } \\
\text { node }\end{array}$} & \multicolumn{3}{|c|}{$16 \times 16$ Fuel } & \multicolumn{3}{c|}{$12 \times 12$ Fuel } \\
\cline { 2 - 7 } & Average & Hot & Hottest & Average & Hot & Hottest \\
\hline 1 & 0.031005555 & 0.000260334 & $1.1602 \mathrm{E}-06$ & 0.031005508 & 0.000259334 & $2.20812 \mathrm{E}-06$ \\
\hline 2 & 0.053908028 & 0.000452632 & $2.01719 \mathrm{E}-06$ & 0.053907946 & 0.000450892 & $3.83917 \mathrm{E}-06$ \\
\hline 3 & 0.078108588 & 0.000655829 & $2.92276 \mathrm{E}-06$ & 0.078108469 & 0.000653308 & $5.56267 \mathrm{E}-06$ \\
\hline 4 & 0.096884792 & 0.000813481 & $3.62535 \mathrm{E}-06$ & 0.096884645 & 0.000810354 & $6.89985 \mathrm{E}-06$ \\
\hline 5 & 0.10710276 & 0.000899275 & $4.00769 \mathrm{E}-06$ & 0.107102597 & 0.000895818 & $7.62755 \mathrm{E}-06$ \\
\hline 6 & 0.116224438 & 0.000975864 & $4.34902 \mathrm{E}-06$ & 0.11622426 & 0.000972113 & $8.27716 \mathrm{E}-06$ \\
\hline 7 & 0.113529456 & 0.000953236 & $4.24817 \mathrm{E}-06$ & 0.113529283 & 0.000949572 & $8.08524 \mathrm{E}-06$ \\
\hline 8 & 0.109992876 & 0.000923541 & $4.11584 \mathrm{E}-06$ & 0.109992708 & 0.000919992 & $7.83337 \mathrm{E}-06$ \\
\hline 9 & 0.095027524 & 0.000797886 & $3.55585 \mathrm{E}-06$ & 0.095027379 & 0.00079482 & $6.76758 \mathrm{E}-06$ \\
\hline 10 & 0.077285799 & 0.00064892 & $2.89197 \mathrm{E}-06$ & 0.077285681 & 0.000646426 & $5.50407 \mathrm{E}-06$ \\
\hline 11 & 0.056348596 & 0.000473124 & $2.10852 \mathrm{E}-06$ & 0.05634851 & 0.000471305 & $4.01298 \mathrm{E}-06$ \\
\hline 12 & 0.031427412 & 0.000263876 & $1.17599 \mathrm{E}-06$ & 0.031427364 & 0.000262862 & $2.23817 \mathrm{E}-06$ \\
\hline
\end{tabular}

\subsubsection{Accident Scenarios and Acceptance Criteria}

The objective of the safety analysis is to demonstrate that the reactor fueled with FCM fuel meets the safety requirements and acceptance criteria during anticipated operational occurrences (AOOs) or accidents. Accidents are further subdivided into DBAs, BDBAs, and severe accidents (SAs) according to their frequency of occurrence and acceptance criteria of integrity of the barriers. Although the full spectrum of possible scenarios, from transients to DBAs to SAs, should be considered to assess the safety effect of the FCM-fueled core, at this stage typical accident scenarios of each category have been selected to assess the accident tolerance of FCM-fueled core.

The selected AOO scenario is a loss of coolant flow transient to evaluate the DNB margin of FCM-fueled core. As a limiting DBA scenario, the LBLOCA with safety injection is selected to check the integrity of the fuel cladding material. The REA is selected to check the PCMI integrity of FCM fuel. In addition to DBA events, BDBA events such as SBO and LBLOCA without a safety injection scenario are considered to evaluate the accident-tolerant envelope of the FCM fuel.

The fuel integrity criteria used for the safety analysis of nuclear power plants (NPPs) are presented as Table 16.

The current acceptance criteria of $\mathrm{UO}_{2} /$ Zircaloy fuel are well specified in regulatory documents; however, the FCM acceptance criteria during accidents are not well known yet. In this phase, the limit for stainless steel clad integrity for LOCAs was chosen as 1530K according to NUREG-0065 (Coffman, 1976). The energy deposition criteria for FCM fuel integrity during REA accident were chosen to be the same as for $\mathrm{UO}_{2}$ fuel. The melting temperature of FCM was selected as $2730 \mathrm{~K}$, which is the melting point of SiC. These interim acceptance criteria should be replaced with updated criteria through the coordinated experimental program in future stages. 
Table 16. Event categorization and acceptance of fuel integrity

\begin{tabular}{|c|c|c|c|}
\hline $\begin{array}{l}\text { ANS Event } \\
\text { Category }\end{array}$ & $\begin{array}{l}\text { New } \\
\text { Regulatory } \\
\text { Category }\end{array}$ & $\begin{array}{l}\text { Fuel Integrity Criteria } \\
\mathrm{UO}_{2} \text { Fuel /Zircaloy clad }\end{array}$ & $\begin{array}{c}\text { Fuel Integrity Criteria } \\
\text { FCM Fuel }\end{array}$ \\
\hline $\begin{array}{l}\text { Moderate } \\
\text { Frequency }\end{array}$ & \multirow{2}{*}{$\mathrm{AOO}$} & $\begin{array}{l}\text { No Fuel Failure } \\
\qquad(\mathrm{DNBR}>\mathrm{SAFDL}\end{array}$ & \multirow{2}{*}{$\begin{array}{l}\text { No Fuel Failure } \\
(\text { DNBR }>\text { SAFDL } \\
\text { LHR }<\text { SAFDL) } \\
\text { SAFDL = Specified } \\
\text { Acceptable Fuel Design Limit }\end{array}$} \\
\hline $\begin{array}{l}\text { Infrequent } \\
\text { Incidents }\end{array}$ & & $\begin{array}{l}\text { SAFDL }=\text { Specified Acceptable } \\
\text { Fuel Design Limit }\end{array}$ & \\
\hline Limiting Faults & DBA & $\begin{array}{l}\text { Coolable geometry } \\
\mathrm{T}_{\text {clad }}<1470 \mathrm{~K} \\
\text { Local Oxidation }<17 \% \\
\text { PCMI : Fuel energy }<230 \mathrm{Cal} / \mathrm{g} \\
\text { No center line melting }<3113 \mathrm{~K}\end{array}$ & $\begin{array}{l}\text { Coolable Geometry } \\
\text { FCM/Stainless steel Clad } \\
\mathrm{T}_{\text {clad }}<1530 \mathrm{~K} \text { (NUREG-0065) } \\
\text { PCMI : Fuel energy }<230 \mathrm{Cal} / \mathrm{g} \\
\text { No center line melting }<2730 \mathrm{~K} \\
\text { FCM/SiC Clad } \\
\mathrm{T}_{\text {clad }}<2750 \mathrm{~K} \\
\mathrm{PCMI}: \text { Fuel energy }<230 \mathrm{Cal} / \mathrm{g} \\
\text { No center line melting }<2730 \mathrm{~K}\end{array}$ \\
\hline
\end{tabular}

\subsection{PRELIMINARY SCOPING ANALYSIS FOR OPR-1000}

\subsubsection{Steady State Performance}

Table 17 shows the comparison of major steady state parameters of the OPR-1000 fueled by different types of FCM fuel assemblies.

\section{6×16 FCM with SS Clad}

Since the FCM fuel rod diameter for the $16 \times 16$ grid is slightly larger than the reference OPR-1000 fuel, the wall friction of the core is slightly increased. Although the pump flow rate is slightly decreased because of the increased core friction, the core mass flux is increased by the reduced core flow area. The MDBNR is increased by the increased mass flux and surface area. The centerline temperature is reduced by the high conductivity of the $\mathrm{SiC}$ fuel matrix.

\section{6×16 FCM with SiC Clad}

Since the fuel dimensions are the same as the SS clad case, all parameters are same as in the previous case except the fuel centerline temperature. The centerline temperature is increased by the lower conductivity of the SiC clad.

\section{$12 \times 12$ FCM with SS Clad}

Since the $12 \times 12$ FCM fuel rod diameter is larger than OPR-1000 fuel, the wall friction of the core is increased. The core mass flux is increased by the reduced core flow area. The MDNBR is decreased by the net effect of increased heat flux. The centerline temperature is reduced by the high conductivity of the $\mathrm{SiC}$ fuel matrix. 


\section{$12 \times 12$ FCM with SiC Clad}

All parameter values are the same as for the $12 \times 12$ SS clad case, except for the fuel centerline temperature, which is increased by the lower conductivity of the $\mathrm{SiC}$ clad.

Table 17. Major parameter of OPR-1000 fueled by the different fuel assembly types

\begin{tabular}{|l|c|c|c|c|c|}
\hline Plant Parameter & $\begin{array}{c}\text { Original } \\
\text { OPR-1000 }\end{array}$ & $\begin{array}{c}16 \times 16 \mathrm{FCM} \\
\text { with SS } \\
\text { Clad }\end{array}$ & $\begin{array}{c}16 \times 16 \mathrm{FCM} \\
\text { with SiC } \\
\text { Clad }\end{array}$ & $\begin{array}{c}12 \times 12 \mathrm{FCM} \\
\text { with SS } \\
\text { Clad }\end{array}$ & $\begin{array}{c}12 \times 12 \mathrm{FCM} \\
\text { With SiC } \\
\text { Clad }\end{array}$ \\
\hline Core Power (MWth) & 2815 & 2815 & 2815 & 2815 & 2815 \\
\hline Pump Flow Rate (kg/sec) & 3985 & 3947 & 3947 & 3880 & 3880 \\
\hline Cold leg temperature (K) & 569.6 & 569.5 & 569.5 & 569.5 & 569.5 \\
\hline Hot leg temperature (K) & 599.7 & 599.9 & 599.9 & 600.3 & 600.3 \\
\hline $\begin{array}{l}\text { Hot Assembly Mass Flux } \\
\text { (kg/m }{ }^{2} \text { sec) }\end{array}$ & 3782 & 3901 & 3901 & 4378 & 4378 \\
\hline $\begin{array}{l}\text { Clad Surface Temperature at } \\
\text { hot spot (K) }\end{array}$ & 624.29 & 623.26 & 623.26 & 627.07 & 627.07 \\
\hline $\begin{array}{l}\text { Fuel Centerline Temperature at } \\
\text { hot spot (K) }\end{array}$ & 1692.5 & 1033.6 & 1180.6 & 1184.6 & 1351.8 \\
\hline Surface heat flux (MW/m $\left.{ }^{2}\right)$ & 1.265 & 1.227 & 1.227 & 1.578 & 1.578 \\
\hline MDNBR & 3.02 & 3.12 & 3.12 & 2.63 & 2.63 \\
\hline
\end{tabular}

* DNBR is determined by the AECL Lookup table for global CHF correlation.

The comparison of radial distribution of fuel temperatures for the four FCM fuel cases and the reference OPR-1000 fuel is given in Figure 39. This figure shows the effect of the higher conductivity of the SiC matrix material, and the lower conductivity of the $\mathrm{SiC}$ clad material. The gap conductance of FCM fuel is lower than for the $\mathrm{UO}_{2}$ fuel, since the gap distance is larger than for the $\mathrm{UO}_{2}$ fuel by the much smaller thermal expansion of the FCM fuel pellet. 


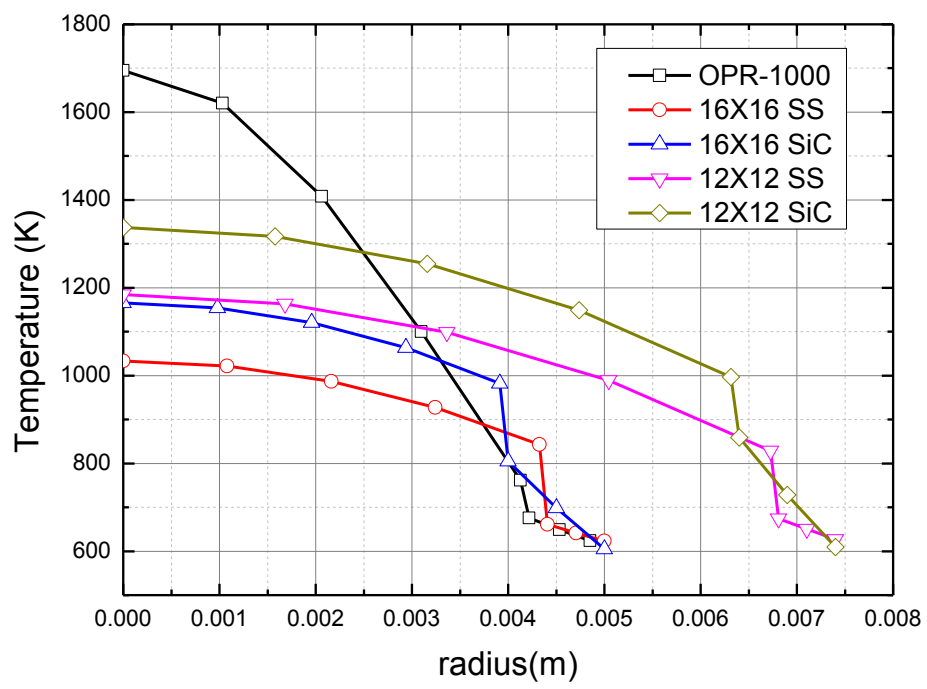

Fig. 39. Radial profile fuel temperature for each fuel type in steady state.

\subsubsection{Loss of Flow Accident}

The complete loss of forced reactor coolant flow can result from the simultaneous loss of electrical power in all reactor coolant pumps. The only credible failure that can result in the simultaneous loss of power is the complete loss of offsite power. Since a loss of offsite power is assumed to result in a turbine trip that would render the steam dump and bypass systems unavailable, the plant cooldown can only be performed by utilizing secondary and atmospheric valves. A total loss of forced reactor coolant flow produces a minimum DNBR that is more adverse than any partial loss of a forced reactor coolant flow event. The major parameter of concern for loss of reactor coolant flow events is the minimum hot channel DNBR.

Figure 40 presents the minimum DNBR trends for each FCM fuel case. The effect of the fuel rod arrangement in the fuel assembly on the DNBR trend is dominant. A $12 \times 12$ fuel rod arrangement has a lower minimum DNBR than the other cases. Because a $12 \times 12$ fuel rod arrangement has fewer rods in the core than $16 \times 16$ geometry, the power per rod is greater, resulting in a lower minimum DNBR. The minimum DNBR is however around 2.3, which still meets the safety criteria. The 16×16 FCM fuel on the other hand has a higher minimum DNBR than the reference conventional fuel because of its larger fuel rod diameter and consequent lower heat flux. 


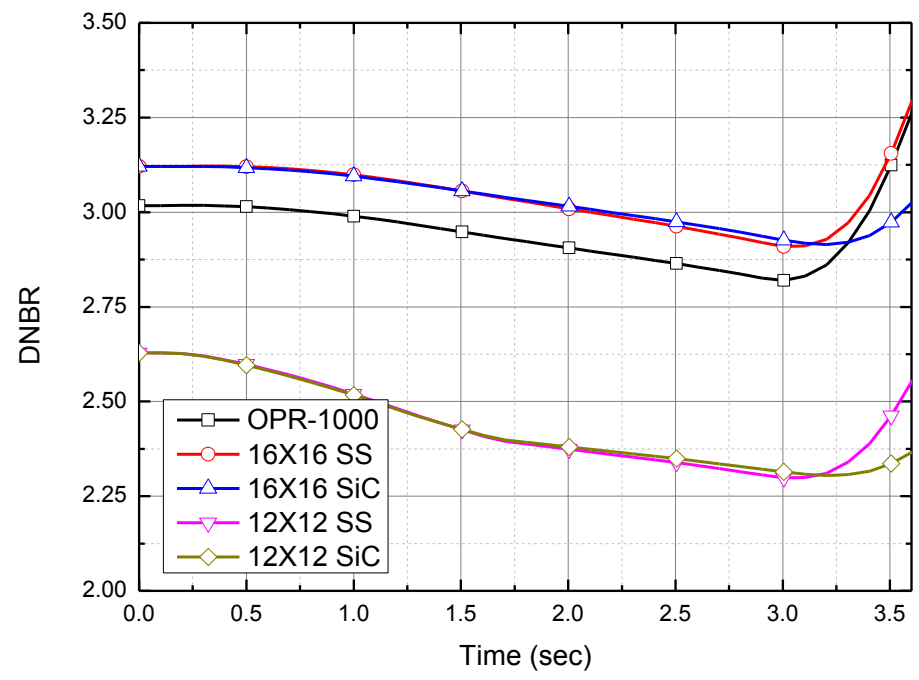

Fig. 40. DNBR for hottest rod in loss of flow accident.

\subsubsection{Large-Break Loss of Coolant Accident}

LBLOCA is the main design basis accident for with OPR-1000. A hypothetical double-ended guillotine break in a cold leg pipe was considered. The safety injection system of OPR-1000 consists of two trains of high-pressure safety injection (HPSI) and two trains of low-pressure safety injection (LPSI). In this study, a single failure of the diesel generator was considered. Thus, only one HPSI and one LPSI were activated. The major parameter of concern is the peak cladding temperature of the hottest fuel rod.

The LBLOCA analysis results are shown in Figure 41. A larger initial stored energy $(12 \times 12$ FCM with $\mathrm{SiC}$ clad) results in a higher peak cladding temperature. SiC cladding has lower thermal conductivity than stainless steel, which is why the case using SiC cladding has a higher peak cladding temperature. However, all cases are bounded by the criteria described in Section 5.2.3.

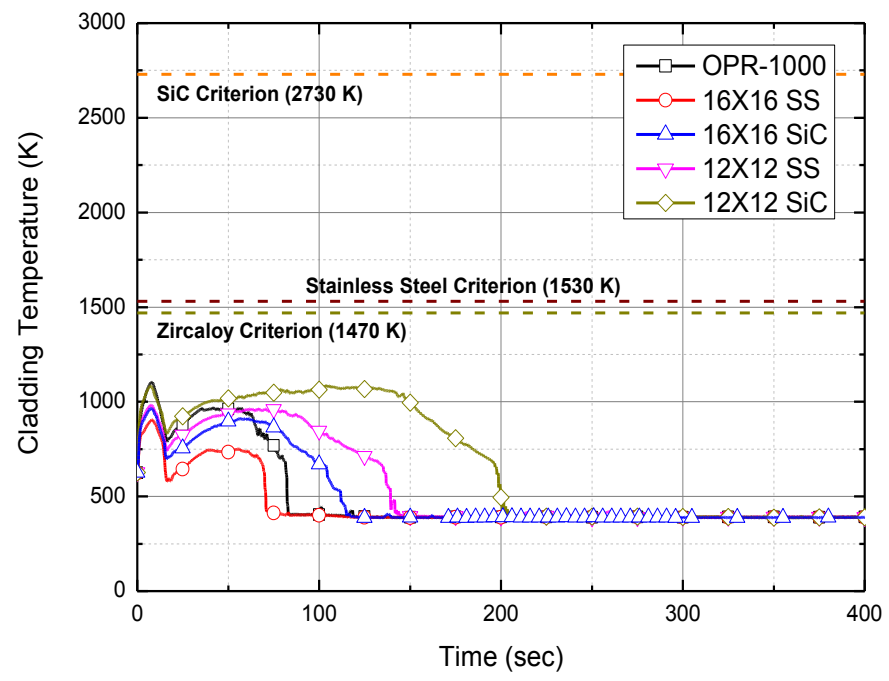

Fig. 41. Cladding temperature for hottest rod in loss of coolant accident. 


\subsubsection{Rod Ejection Accident}

The REA event is initiated by the loss of integrity of the CR drive housing with rapid expulsion of a control assembly from the core due to the differential pressure between primary coolant and the containment. This event leads to a rapid reactivity insertion. The REA safety aspects are as follows.

(a) Rapid reactor power increase resulting in a fuel temperature rise and in a reduction of DNBR; hence, a large reduction of heat removal is possible, with subsequent fuel rod damage and radioactivity release.

(b) The radially averaged fuel pellet enthalpy should not exceed prescribed values (the values differ significantly among different reactor designs and depend also on fuel burnup) at any axial location of any fuel rod during the REA. This criterion ensures that fuel integrity is maintained and energetic fuel dispersion into the coolant will not occur.

While the transient 3D neutronic analysis is preferable, a combination of the 3-D steady state power distribution analysis with a transient point kinetics calculation of the total reactor power is acceptable for the sensitivity analysis. The kinetic parameters for the FCM-fueled core have not yet been determined, and the acceptance criteria for FCM fuel integrity are not established. In this phase, the sensitivity analysis of different FCM fuel configurations has been performed at full power, assuming the reactivity insertion rate and feedback effect of FCM are similar to $\mathrm{UO}_{2}$ fuel.

One of the main acceptance criteria is a radially averaged pellet enthalpy not exceeding prescribed values. Therefore the fuel-averaged temperatures have been plotted in Figure 42 for all the FCM fuel configurations and the reference standard fuel. The maximum averaged fuel temperatures of the $16 \times 16$ FCM fuel are significantly lower than for the $\mathrm{UO}_{2}$ fuel because of the higher conductivity of the FCM fuel pellet. The thermal behavior of the $12 \times 12 \mathrm{FCM}$ fuel is similar to $\mathrm{UO}_{2}$ fuel because of its similarly high initial stored energy. The maximum fuel centerline temperatures for all the FCM fuels are low enough to prevent the fuel from melting. Although the results are indicative of increased accident tolerance for the FCM fuels, different PCMI mechanisms are expected for the FCM fuels, and the acceptance criteria might be different from those of $\mathrm{UO}_{2}$ fuel. The true safety margin of FCM fuel against REA should be determined by the coordinated experiment validation program for FCM fuel.

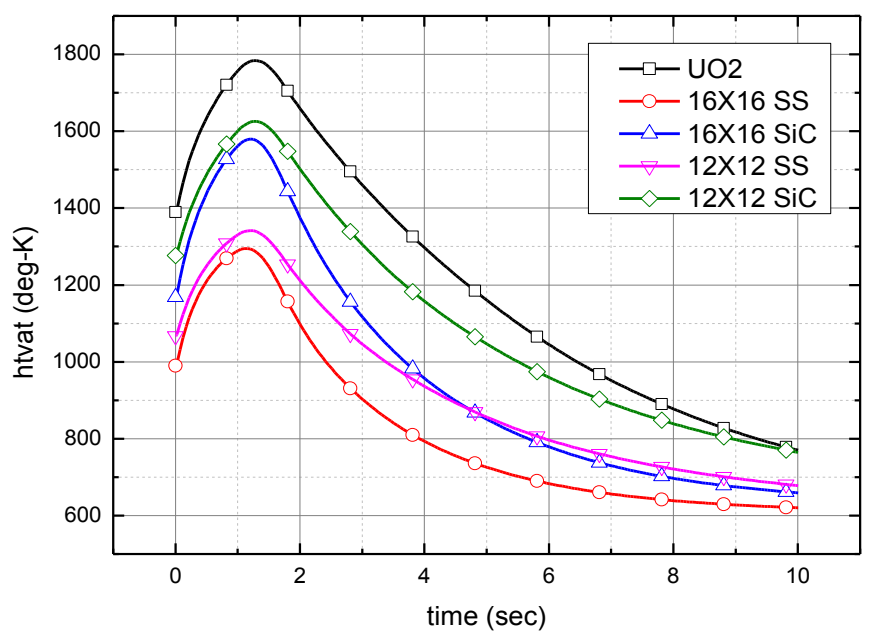

Fig. 42. Averaged fuel temperature for hottest rod in rod ejection accident. 


\subsubsection{Station Blackout}

The SBO event is an accident caused by the loss of all available electric power, including offsite power, emergency diesel power, and alternative $\mathrm{AC}$ power. The reactor, turbine, reactor coolant pump, and main feedwater are cut off because of the loss of offsite power. Because AC power fails, none of the HPSIs and LPSIs can be injected. Only the accumulator is activated. In a SBO event, the loss of heat sink and coolant due to the opening of the pressurizer safety valve will rapidly increase the peak cladding temperature to failure levels.

In the SBO event, the lack of active safety injection causes the cladding and fuel temperatures to increase continuously. The safety concern is the time at which radioactive material is released. For the Zirc-alloy cladding reference fuel, radioactive material is released when a coolable geometry can no longer be maintained by the cladding failure. On the other hand, for the FCM fuel, radioactive material is contained in the fuel pellet even after the cladding failure, because the SiC layer of the TRISO and the SiC pellet matrix are still intact as a radiation barrier. As shown in Figure 43, the FCM fuel has a margin of 100 minutes longer than the conventional $\mathrm{UO}_{2}$ fuel for operator actions.

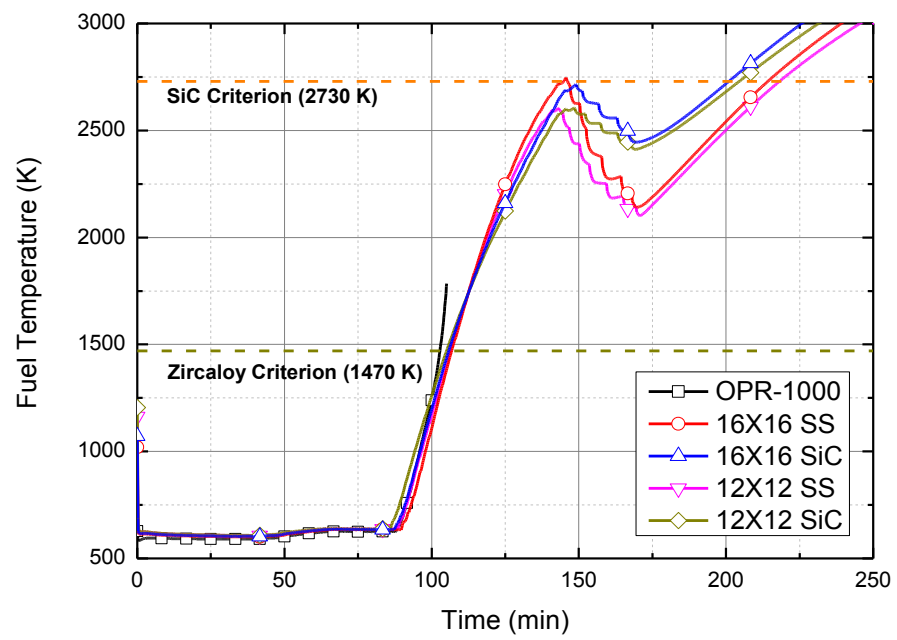

Fig. 43. Fuel temperature for hottest rod in station blackout.

\subsubsection{LBLOCA without Safety Injection}

The LBLOCA without safety injection (SI) is considered to be a BDBA event. While one HPSI and one LPSI are activated in the design basis LBLOCA, no SI system is assumed available in this extreme scenario. Without the active inventory makeup, the core is depleted and heats up rapidly. The heat-up rate of the Zirc-alloy conventional fuel is quite faster than the FCM fuel by the added metal-water-reaction heat. For the conventional fuel, radioactive material is released when the Zirc-alloy cladding fails by heatup. On the other hand, the rate of heat-up is slower for the FCM fuel. In addition, by virtue of the refractory $\mathrm{SiC}$ layer of TRISO and $\mathrm{SiC}$ pellet matrix, radioactive material is retained longer in the fuel pellet even after the cladding failure. As shown in Figure 44, the FCM fuel has a margin of 1000-1200 seconds (up to 20 minutes) over the reference fuel. 


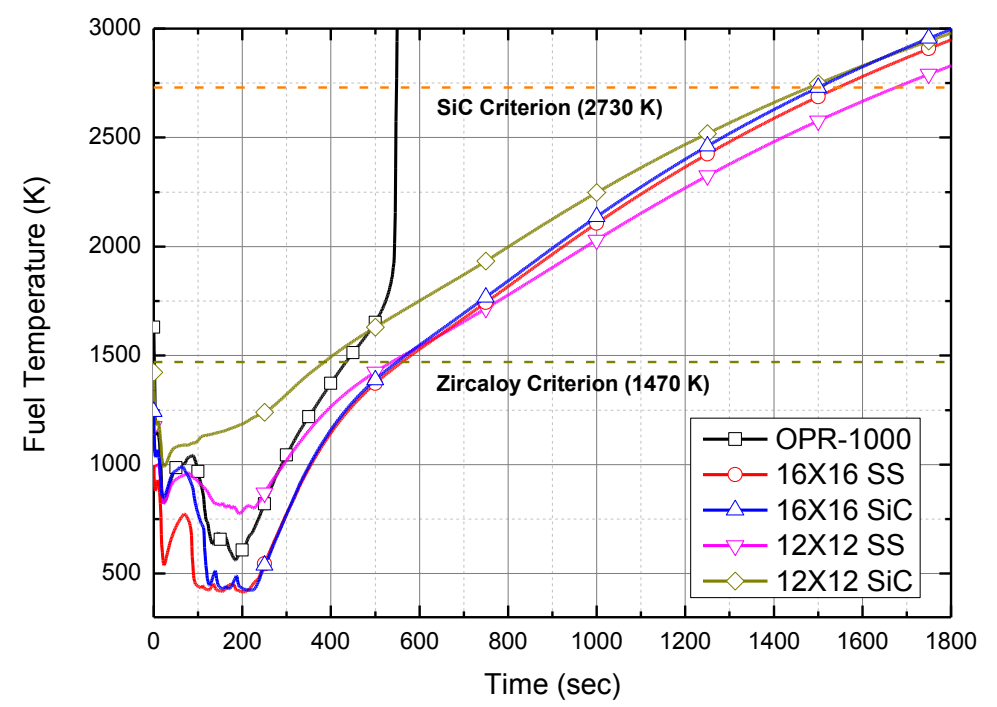

Fig. 44. Cladding temperature for hottest rod in LBLOCA without safety injection.

\subsection{PLANNED ACTIVITIES FOR NEXT YEAR}

\subsubsection{Scoping Analysis for OPR-1000}

The following topics will be covered in the next year.

- Development of Safety Analysis Methodology for Transition Core

- Scoping Analysis DBA/BDBA Scenarios

- Consideration of Radiation Heat Transfer in Beyond DBA

- Consolidation of Safety Analysis System

- Coupled core neutronics, core thermal-hydraulics, and system thermal-hydraulics analysis

\subsubsection{Scoping Analysis for WH Plants}

The following topics will be covered in the next year.

- Development of Safety Analysis Methodology for Transition Core

- Scoping Analysis DBA/BDBA Scenarios

\subsection{SUMMARY}

In order to evaluate the feasibility of FCM fueled core, preliminary safety analysis was performed using fuel-assembly-level physics parameters. FCM fuel designs analyzed are the $12 \times 12$ and 16×16 fuel assembly arrays with $\mathrm{SiC}$ or SS cladding. MARS system thermal-hydraulics code is used for the analysis of limiting accident scenarios for DBAs and BDBAs, where preliminary safety criteria for the FCM fuel are proposed and applied. In addition, the MARS-MARSTER coupled safety analysis system has been developed for more detailed coupled analysis in the second year.

Limiting DBA scenarios are analyzed for LOFA, LOCA, and REA, and it is found that the safety of the FCM-fueled core meets preliminary safety criteria with sufficient margin. Analysis results of the extreme BDBA scenarios such as LOCA without SI and SBO show that the FCM fuel pellet in the SiC matrix can survive considerably longer than the conventional $\mathrm{UO}_{2}$ and thus increase the time frame for mitigating actions by plant operators. 


\subsection{REFERENCES}

Coffman Jr., F.D. 1976. LOCA Temperature Criterion for Stainless Steel Clad Fuel, NUREG-0065, 1976.

KAERI, 2006. MARS CODE MANUAL VOLUME I: Code Structure, System Models, and Solution Methods, KAERI/TR-2812/2004.

Snead, L.L., et al., 2007. Handbook of SiC properties for fuel performance modeling," Journal of Nuclear Materials, 371, 329-377 (2007).

Snead L.L., et al., 2012. Fully Ceramic Microencapsulated Fuel Development for LWR Applications, TOPFUEL 2012. 


\section{FUEL QUALIFICATION}

\subsection{TASK DESCRIPTION}

The experimental qualification of FCM fuel performance, by irradiation and post-irradiation examination and subsequent iterations in fabrication recipes and manufacturing techniques, is required to confirm the FCM fuel performance within the limiting envelope of design basis accidents as well as normal operations. A computer model to assess the FCM fuel behavior must also be developed for analytic prediction of performance of the FCM fuel. The fuel qualification task (Task 4) consists of the following subtasks.

- Manufacturing of FCM fuel samples

- Irradiation of FCM fuel

- Post-irradiation examination of FCM fuel

- Modeling of reference FCM fuels (TRISO models + LWR fuel/clad/coolant models)

- Fuel performance analysis during accidents (starting with the equilibrium core)

Manufacturing, irradiation, and post-irradiation examination of the FCM fuel will be performed at ORNL. KAERI and USNC will produce a practical model of fuel performance for the FCM fuel, integrating existing TRISO fuel models with LWR fuel/clad and clad/coolant models.

In the first year, FCM UCO fuel samples are manufactured and irradiated in the ORNL's High Flux Isotope Reactor (HFIR) facility. TRISO particle fuel performance models under LWR operating conditions are set up and applied for preliminary scoping analysis for fuel design selection.

\subsection{FUEL QUALIFICATION}

\subsubsection{Manufacturing of Fully Ceramic Microencapsulated Fuel Sample}

\section{Process Basics and Consolidation}

Fully ceramic microencapsulated fuels consist of TRISO fuel particles embedded in a SiC matrix, (Figure 45) with their manufacture first described by Snead in 2010 (Snead et al., 2011) and more rigorously presented by Terrani in 2012 (Terrani et al., 2012a). A TRISO particle consists of a spherical fuel kernel that is coated with successive layers of porous carbon (buffer layer), a dense inner pyrocarbon (IPyC), CVD SiC, and an outer pyrocarbon (OPyC) layer. Over the past five decades, TRISO fuel particle technology has been developed and optimized for gas reactors now capable of delivering remarkable levels of performance with respect to fission product retention, high-temperature performance, and fissile burnup. The newly invigorated and improved TRISO technology has enabled fuel and reactor designers to extend the application of TRISO particles to other fuel and reactor systems. In conventional hightemperature gas-cooled reactor (HTGR) applications, the TRISO particles are dispersed in a graphitic matrix, producing compacts in the form of pebbles or pellets (Nickel et al., 2002; Phillips et al., 2010). Under the FCM fuel concept, the graphite matrix is replaced with a SiC matrix that offers the following potential advantages: (i) improved irradiation stability (Snead et al., 2007; Snead et al., 2008), (ii) incorporation of yet another effective barrier to fission product release, (iii) environmental stability under operating (steady state) and transient conditions as well as long-term storage, and (iv) proliferation resistance. The high thermal conductivity of the graphite matrix is also matched by the SiC matrix (Snead et al., 1995). Details regarding the advantages and motivations behind the FCM fuel concept are discussed more extensively in a companion paper (Terrani et al., 2012b). Specific discussion of standard TRISO fabrication has been well presented and is available elsewhere (Hunn et al., 2006; Hunn et al., 2010). 


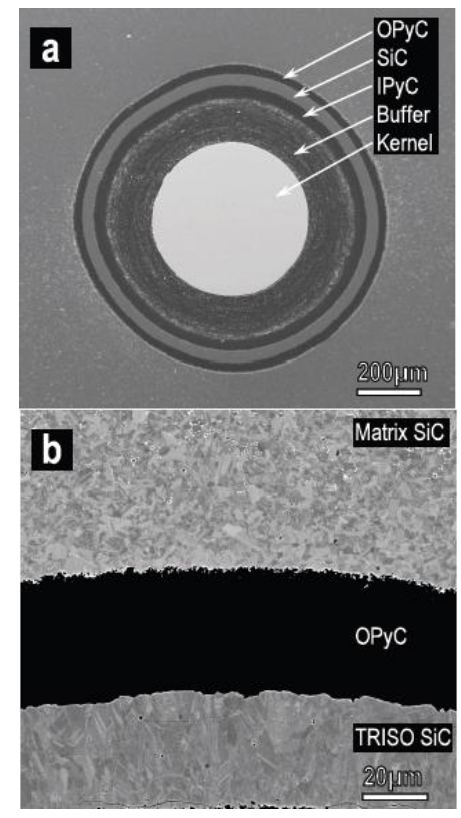

Fig. 45. (a) Secondary electron image of a TRISO particle embedded in the SiC matrix in an FCM pellet; (b) backscattered electron image of TRISO particle matrix $\mathrm{SiC}$ interface where the $\mathrm{SiC}$ grain structure in the particle and the matrix are in Snead et al. (2011).

The Nano-Infiltration and Transient Eutectic-phase (NITE) processing of SiC is a specific type of liquidphase sintering (LPS) (Omori et al., 1982) utilizing SiC nanopowder with a limited amount of oxide additives to produce near fully dense SiC (Zhou et al., 2001; Katoh et al., 2002b; Dong et al., 2003). The oxide additives generally consist of alumina-rare earth oxides mixed at eutectic compositions, reducing the typical required processing temperature by about $200-400^{\circ} \mathrm{C}$ (Shimoda et al., 2007). The process was originally developed for fiber-reinforced SiC-matrix composite fabrication for nuclear applications, with research continuing to this day (Shimoda et al., 2009a; Shimoda et al., 2009b). The main motivation for the early work in this area was elimination of fiber damage during processing while achieving high strength and reasonable thermal conductivity. Lowering the $\mathrm{SiC}$ sintering temperature is of great importance when incorporation of fibers, or in this case TRISO fuel particles, is intended. This is necessary since temperatures above $1900^{\circ} \mathrm{C}$ could result in major crystal growth in $\mathrm{SiC}$ fibers or grain growth in the $\mathrm{SiC}$ layer in TRISO particles, resulting in unacceptable deterioration in mechanical properties (Sha et al., 2004). The resulting NITE SiC microstructure is significantly, and importantly, different from that of conventional LPS. Specifically, the SiC powder feedstock for fabrication of conventional LPS is initially in the micrometer size range and does not experience significant growth. Therefore, the resulting microstructure consists of relatively large islands of $\mathrm{SiC}$ embedded in a continuum of oxide-phase grain boundary sintering aids. In contrast, during the NITE process, significant solution-reprecipitation of the $\mathrm{SiC}$ takes place to achieve $\mathrm{SiC}$ grains up to a few micrometers in size with feedstock SiC powder material that ranges in tens of nanometers in size. Effectively, the NITE process yields dense $\mathrm{SiC}$ materials that require significantly smaller mass of oxide additives. Limiting the extent of impurities in the $\mathrm{SiC}$ matrix in this manner in turn results in a final material with radiation evolution characteristics approaching that of chemical vapor-deposited (CVD) $\mathrm{SiC}$, which is highly radiation resistant. After consolidation of the matrix by NITE process, the oxide additives precipitate as a mixture of glass and crystalline phases at the triple junctions and boundaries between $\mathrm{SiC}$ grains. Early studies on the irradiation behavior of NITE SiC in neutron environments (Ozawa et al., 2009) or under silicon ion bombardment (Koyanagi et al., 2011) suggest limited swelling, approaching that of CVD SiC. Detailed characterization of the samples in these studies suggested that while the SiC grains undergo swelling 
similar to CVD SiC as a function of temperature and dose, the oxide phases experience larger volumetric expansions. The extent of swelling is reported as more severe in the yttrium-rich oxide phase. As will be seen in the next section, swelling of the NITE at these temperatures appears to behave in a similar fashion as the model CVD SiC. Utilization of the NITE process for production of FCM fuels offers the following benefits: (i) damage to the TRISO particle through particle-powder mechanical interaction is avoided since the powder is in the nanometer size range, (ii) a high-density SiC matrix is achieved that offers relatively high thermal conductivity, high strength, and an effective secondary barrier to fission product release, (iii) a highly crystalline form of $\mathrm{SiC}$ which has been demonstrated to be stable under neutron irradiation, and (iv) a versatile fabrication process whereby a variety of geometries could be fabricated and/or subsequently bonded together through repetition of the NITE process.

The powder mixtures utilized for this particular FCM fuel fabrication consisted of SiC nanopowder (Nanostructured \& Amorphous Materials, Inc., lot \#4620-123109) with addition of yttria (Nanostructured \& Amorphous Materials Inc., lot 5610-091410), alumina (Nano Products Corp., lot 20100905), and silica (Nanostructured \& Amorphous Materials Inc, lot 4830-081810). Additionally, a number of materials have been made using alternative (burnable poison) oxides such as yttria and gadolinia. The mean diameter of all the powder particles was $40 \mathrm{~nm}$, as reported by the manufacturers. Transmission electron microscope (TEM) images of the feed $\mathrm{SiC}$ powder are shown in Figure 46, where the presence of oxides on the surface is apparent. Silica or silicon oxycarbide species on the surface of the SiC nanopowder have been previously characterized (Park et al., 2007). The variation in powder size proved to be broad, with particles larger than $100 \mathrm{~nm}$ present. Thorough mixing of the oxide additives with the base SiC powder is necessary to achieve optimized matrix microstructures. While in earlier tests, ball-milling the mixture of powder suspended in an alcohol-based solvent was utilized (Shimoda et al., 2007), in these studies the addition of dispersant agents along with ultrasonic mixing was used to effectively mix the powders. Three different molecular weights $(600,1800$, and 10,000 amu) of the dispersant polyethyleneimine (PEI) were examined. By measuring the zeta potential (Brookhaven Instruments ZetaPALS zeta potential analyzer) of the individual powders and again as a mixture in solution as a function of $\mathrm{pH}$, the overall suspension stability of the matrix and relative degree of powder mixing can be evaluated (Hunter, 1988). Results of the zeta-potential measurement on the single component powders in deionized (DI) upon addition the efficacy of the dispersing agent is evident. 

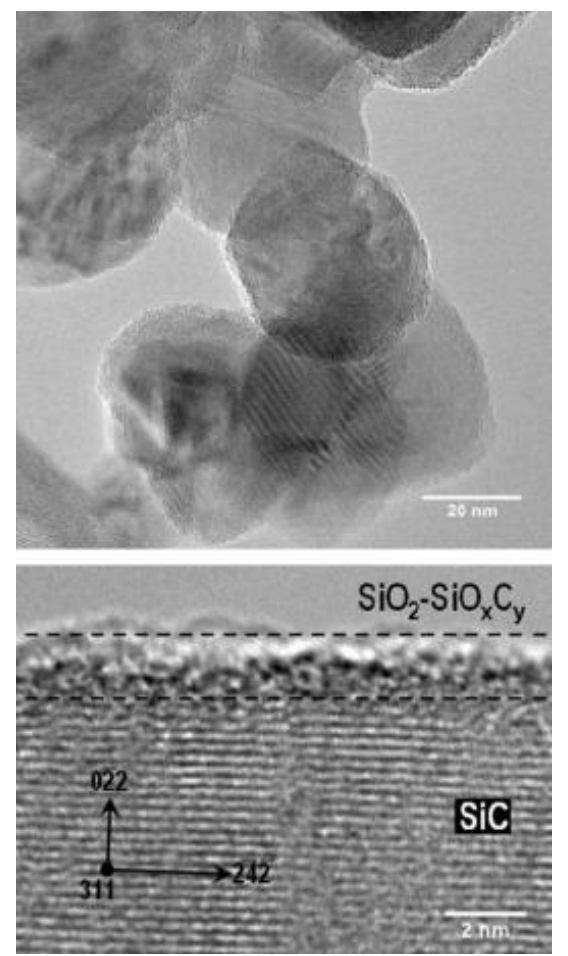

Fig. 46. High-resolution TEM images of (top) $\mathrm{SiC}$ nanopowder suspended on a carbon film and (bottom) thin oxide and oxycarbide layer on the surface of the $\mathrm{SiC}$ nanopowder.

Similar observations were made when the powder mixtures were dispersed in ethanol. This is in agreement with previously published observations in the literature (Sun et al., 2001; Shimoda et al., 2008). Note that the isoelectric point (IEP) of the additive oxide powders is at or below $\mathrm{pH} 8$. This indicates that a cationic dispersant such as PEI is a viable candidate as a dispersant in this system. The addition of PEI at all molecular weights characterized shifts the IEP to greater than $\mathrm{pH} 8$, indicating that the PEI has been adsorbed onto the surface of the powders. Figure 46 shows that addition of PEI, irrelevant of its molecular weight, proves effective in dispersing the nanopowder mixtures. Once the overall efficacy of PEI as a dispersing agent was established, a series of tests to analyze particle size distribution (PSD) were performed (Horiba LA-950 Laser Diffraction Particle Size Distribution Analyzer). During these tests, and throughout the rest of the experimental fabrication work discussed, the use of the PEI dispersing agent was limited to that with a molecular weight of $1800 \mathrm{amu}$ where its concentration was reported as a mass fraction of solid powder. The solvent was ethanol at a constant $6.5 \mathrm{wt} \%$ ratio of the powder mixture to solvent. Ultrasonic mixing was delivered to $20 \mathrm{ml}$ suspensions with a probe (Cole-Parmer, CP-130) operating at roughly $10 \mathrm{~W}$. The first set of analyses focused on the effects of sonication time on SiC PSD in the absence and presence of PEI. In the absence of PEI, sonication partially dispersed the powder particles with continued improvements upon extension of this process to longer duration. In the presence of PEI, powder particles were readily dispersed from the large agglomerates into much smaller agglomerates. The extent of the dispersion process in presence of PEI quickly saturated upon exceeding $5 \mathrm{~min}$ of sonication time. The samples were again analyzed to examine any sedimentation after 5 days; reagglomeration had occurred in the samples without any PEI, while the samples with dispersant remained deagglomerated. The next set of PSD analyses focused on the critical concentration of PEI required to achieve a well-dispersed powder solution in ethanol. In this study the duration of ultrasonic mixing was fixed at $5 \mathrm{~min}$ while the PEI concentration was varied from 0.25 to $3 \mathrm{wt} \%$. It was observed that effective dispersion took place at a PEI concentration above $0.5 \mathrm{wt} \%$. After $24 \mathrm{~h}$, the PSD was reanalyzed and it was found that reagglomeration had taken place for the $0.25 \mathrm{wt} \%$ and 
$0.5 \mathrm{wt} \%$ PEI concentrations. The most well-dispersed distribution after $24 \mathrm{~h}$ was observed for a $1.5 \mathrm{wt} \%$ PEI concentration, indicating that excess PEI could prove detrimental to dispersion stability. Utilization of the dispersing agent to reduce the mean agglomerate size by roughly an order of magnitude has resulted in agglomerate volume reduction of three orders of magnitude. This ensures effective dispersion of oxide additives within the $\mathrm{SiC}$ powder particles to optimize the LPS process. However, it is evident in both of the above analyses that the mean of the PSD in the case of well-dispersed powders deviates from the $40 \mathrm{~nm}$ mean particle size reported earlier. This could suggest the presence of smaller agglomerates consisting of only a few powder particles. The small agglomerates shall prove inconsequential for the purposes of the $\mathrm{SiC}$ matrix fabrication since the mean diameter of $\mathrm{SiC}$ grains after hot pressing is on the order of few micrometers.

\section{Fabrication by Hot Pressing}

Pellet fabrication was carried out by hot pressing the powder-TRISO particle mixtures in a graphite die at elevated temperatures. The majority of fabrication trials were performed at a maximum temperature of $1850^{\circ} \mathrm{C}$; however, deviations of $50^{\circ} \mathrm{C}$ above and below this value were also explored. Higher

temperatures are desirable to achieve a higher degree of densification in the matrix while fabrication at temperatures above $1900^{\circ} \mathrm{C}$ could potentially compromise the properties of the $\mathrm{SiC}$ shell in the coated fuel particle. A constant furnace temperature ramp rate of $20^{\circ} \mathrm{C} / \mathrm{min}$ was used under an inert argon gas environment at $60 \mathrm{kPa}$ of gas pressure. The axial pressure during hot pressing induced by the ramp was varied from 10 to $20 \mathrm{MPa}$.

Among the various oxide sintering aid mixtures, a eutectic temperature exists that is below the final maximum temperature utilized during the hot-pressing process. In the case of a binary alumina-yttria mixture, the lowest eutectic point corresponds to the richest alumina composition and is at $1826^{\circ} \mathrm{C}$ (Mah et al., 1992). The presence of silica on the large surface area associated with the nanopowder or intentional introduction of such among the sintering additives further lowers the liquidus temperature in the oxide mixtures to as low as $1400^{\circ} \mathrm{C}$ (Arita et al., 1992). During the temperature ramp-up, densification in absence of TRISO particles occurs initially due to particle rearrangement, followed by solutionreprecipitation upon formation of the liquid phase, and finally by solid state diffusional processes (Shimoda et al., 2009b). Once the liquid forms, a rapid powder mixture densification rate is readily observable. Figure 47 shows the evolution in temperature profile as well as graphite punch movement during hot pressing as a function of time. The punch movement is representative of the densification process, and its evolution is consistent with the discussion on the onset of liquid-phase formation given above. Note that the punch movement curves have not been corrected for the expansion and contraction of graphite punches upon heating and cooling. Fabrication of FCM pellets over a range of diameters (8$25 \mathrm{~mm}$ ) was carried out with an aspect ratio of unity for most product forms. Fabrication of plates as large as $60 \mathrm{~mm}$ in diameter was also carried out. Utilizing the procedure outlined here, FCM pellets with SiC matrices of full or nearly full density were produced. Figure 48 provides two impressions of TRISO particle distribution in a $\mathrm{SiC}$ matrix. The top image shows a cross section of an FCM pellet (TRISO particle volume fraction >0.4) with typical LWR geometry that was intentionally fractured at the midplane. The lower figure is an X-ray radiographic image of a LWR FCM pellet with 0.3 volume fraction of TRISO particles embedded in the SiC matrix. The contrast in the image clearly distinguishes the zirconia kernels inside the TRISO particles. Note that in the radiography image the OPyC, the $\mathrm{SiC}$, and the IPyC layers of the TRISO particle are not resolvable as they interact essentially the same as the FCM matrix with the incident photons. Consequently, it is only the zirconia phase, consisting of the high-atomicnumber material that stands out in clear contrast in the image. 


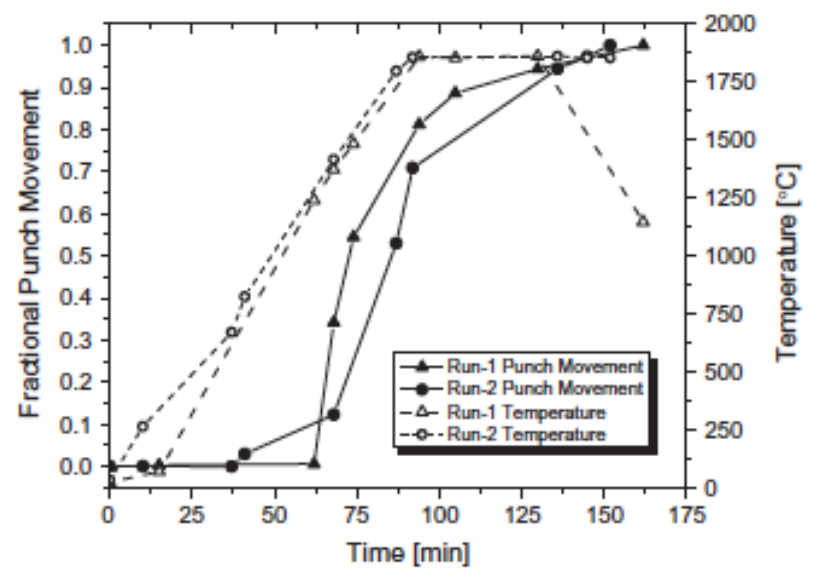

Fig. 47. Fractional punch movement and temperature during hot pressing of FCM pellets with surrogate TRISO particles.
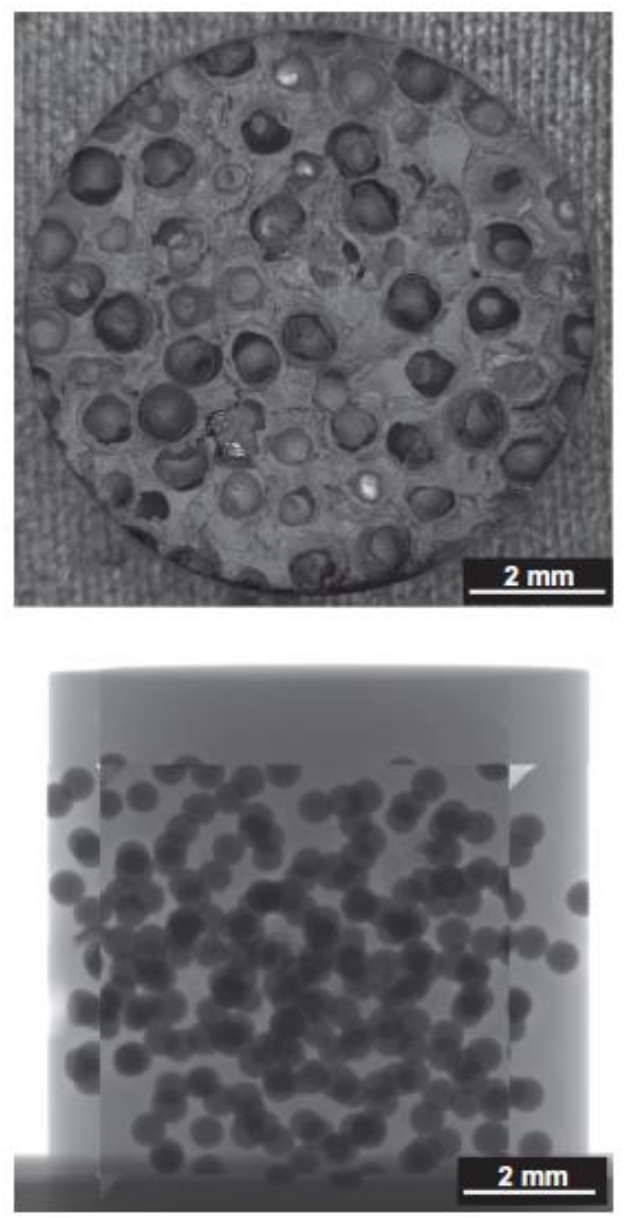

Fig. 48. Top: fracture surface of LWR FCM pellet with $\sim 44$ vol\% TRISO particles and bottom: X-ray radiography of a LWR FCM pellet with 30 vol\% TRISO particles. 


\section{Microstructural Characterization}

The microstructure of the $\mathrm{SiC}$ matrix along with the specific characteristics of the matrix-particle interface could provide extensive information on the expected properties and irradiation behavior of the FCM pellet under LWR operating conditions. Hence, a comprehensive set of results generated through scanning and transmission electron microscopy (SEM and TEM) studies are presented in this section. The SEM study was performed with a JEOL 6500 FEG-type microscope operating at $10 \mathrm{kV}$ accelerating potential. A Hitachi HF3300 instrument was used for the TEM investigations under conventional and scanning modes of operation.

Initially the results of a parametric study are presented that make the case for the advantages of SiC nanopowder over micron- size particles, simply to achieve high-density monolithic SiC materials by hot pressing. Figure 49 shows the microstructure of six $\mathrm{SiC}$ samples hot pressed at $1850^{\circ} \mathrm{C}$ under $20 \mathrm{MPa}$ of pressure for the duration of $1 \mathrm{~h}$. The starting $\mathrm{SiC}$ powder consisted of either the micropowder (mean diameter $0.8 \mathrm{~mm}$ ), the nanopowder (mean diameter $40 \mathrm{~nm}$ ), or mixtures of the two powder sizes.

Table 18 provides detailed information on the composition of the various powder streams for the samples in Figure 49. In the figure, the gray grains with apparent stacking fault lines are $\mathrm{SiC}$, while the extreme dark and bright contrast in the image corresponds to porosity and oxide phase, respectively. The total mass of oxide additives varied among the various specimens, while the mass ratio of $\mathrm{Al}_{2} \mathrm{O}_{3}: \mathrm{Y}_{2} \mathrm{O}_{3}: \mathrm{SiO}_{2}$ in the mixture of oxide additives was maintained at 5:3:2. None of the powder mixtures with $2.5 \mathrm{wt} \%$ oxide additives yielded $\mathrm{SiC}$ specimens with sufficient density. Among the powder mixtures with $5 \mathrm{wt} \%$ oxide additives, only the sample starting solely from nanopowder SiC feed produced a near fully dense specimen (Figure 49e). Finally in an attempt to achieve a high-density specimen with micron-size powder feed, a mixture with $9 \mathrm{wt} \%$ oxide additives was prepared. In this specimen, $\mathrm{SiC}$ grains appeared to be embedded in a continuous matrix of oxide phase and glass (Figure 49f), but the density of the sample was still inferior to that of $\mathrm{SiC}$ nanopowder with $5 \mathrm{wt} \%$ oxide additives. Based on the above observations, $\mathrm{SiC}$ nanopowder with roughly 5-7 wt\% oxide additives was chosen for fabrication of FCM pellets. As was mentioned briefly, an intentional effort has been made in this work to limit the extent of oxide additives needed for SiC densification through utilization of the NITE process.

Table 18. Composition of various powder mixtures used during hot pressing of monolithic SiC specimens

\begin{tabular}{|c|c|c|c|c|c|c|}
\hline Sample ID & $0.8 \mu \mathrm{m} \mathrm{SiC}(\mathrm{wt} \times)$ & $40 \mathrm{~nm} \mathrm{SiC} \mathrm{(wt:})$ & $\mathrm{Al}_{2} \mathrm{O}_{3}(\mathrm{wt} \%)$ & $\mathrm{Y}_{2} \mathrm{O}_{3}(\mathrm{wt} \cdot \mathrm{x})$ & $\mathrm{SiO}_{2}$ (wt: & Total oxide (wt.x) \\
\hline a & 0 & 97.5 & 1.2 & 0.75 & 0.55 & 2.50 \\
\hline b & 95 & 0 & 2.5 & 1.5 & 1.0 & 5.00 \\
\hline c & 57 & 38 & 2.5 & 1.5 & 1.0 & 5.00 \\
\hline d & 38 & 57 & 2.5 & 1.5 & 1.0 & 5.00 \\
\hline e & 0 & 95 & 2.5 & 1.5 & 1.0 & 5.00 \\
\hline f & 91 & 0 & 4.5 & 2.7 & 1.8 & 9.00 \\
\hline
\end{tabular}




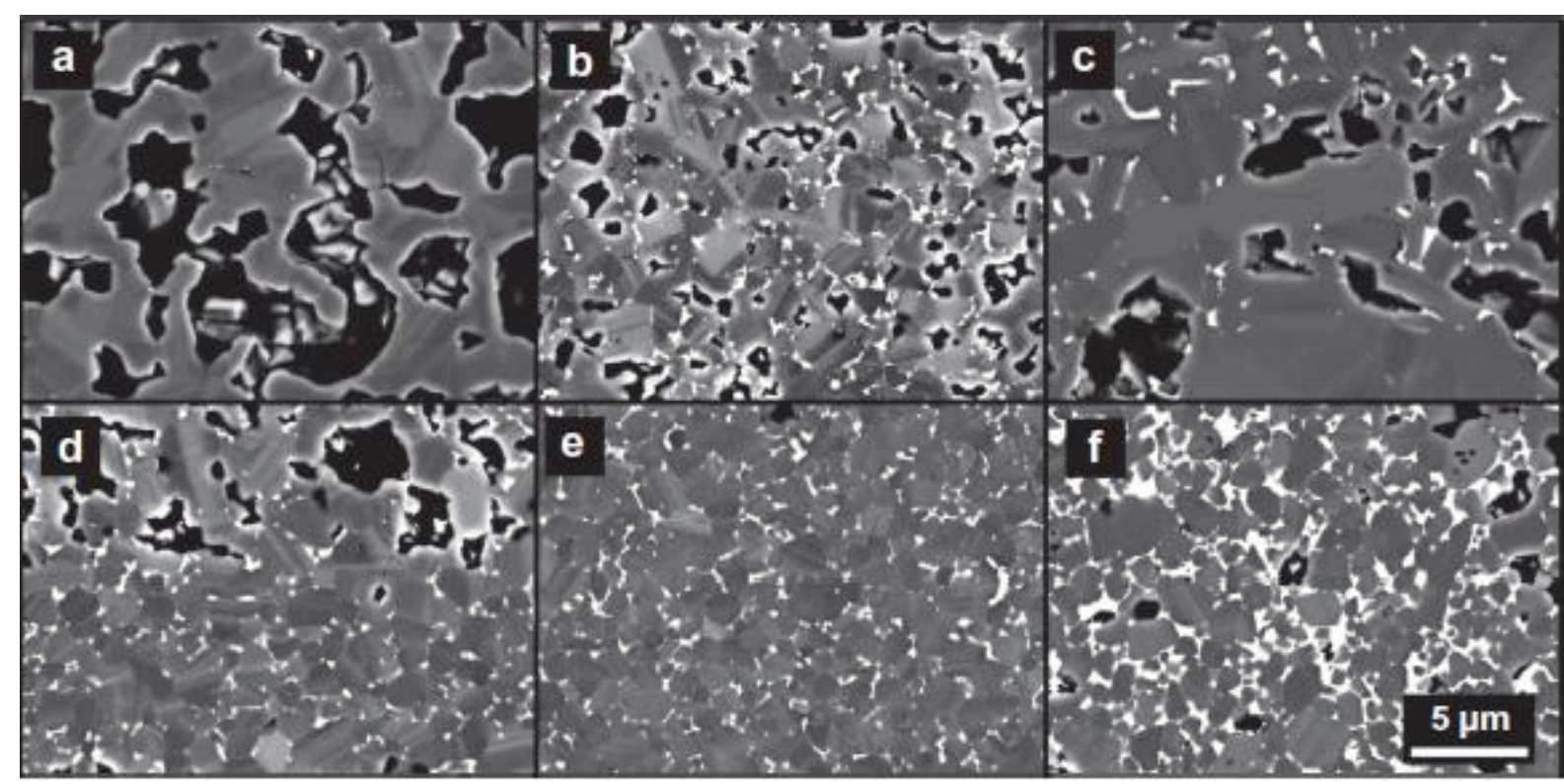

Fig. 49. Backscattered electron image of $\mathrm{SiC}$ specimens hot pressed at $1850^{\circ} \mathrm{C}$ under $20 \mathrm{MPa}$ of pressure for $1 \mathrm{~h}$. The composition of each specimen is given in Table 18.

A TEM study on the SiC matrix was also performed to examine the microstructure in detail and map the distribution of oxide additives in the post-processing condition. Figure 50 captures the NITE SiC microstructure (6 wt\% yttria-alumina) at various magnifications where $\mathrm{SiC}-\mathrm{SiC}$ grain boundaries and a triple junction location between the $\mathrm{SiC}$ grains have been examined in the phase contrast mode.

Figure 50b suggests that while some oxide phase is present at the grain boundaries, it remains very limited in extent. Figure 50c shows a high-resolution image of the grain boundary filled with a thin $(2 \mathrm{~nm})$ amorphous oxide phase. A crystalline alumina precipitate is shown to be present at the triple junction, while the extension of the oxide phase in between the grain boundaries appears to be in the amorphous form. These observations remain consistent with previously reported results (Sigl et al., 1993). Figure 51 shows the results of the TEM examination in the scanning mode along with energy dispersive $\mathrm{X}$-ray spectroscopy (EDS). The EDS map confirms the presence of oxide species at the grain boundary. The yttria-alumina binary-phase diagram (Mah et al., 1992) suggests that the crystalline oxides in the post-processing condition should consist of a mixture of $\mathrm{YAG}\left(\mathrm{Y}_{3} \mathrm{Al}_{5} \mathrm{O}_{12}\right)$ and alumina. Also since some silica was present at the surface of the $\mathrm{SiC}$ nanopowder, formation of an alumina-silicate phase is also possible. This is in agreement with the EDS map of Figure 50c, where yttrium-aluminum oxides along with a single alumina phase fill the triple joints between $\mathrm{SiC}$ grains. 


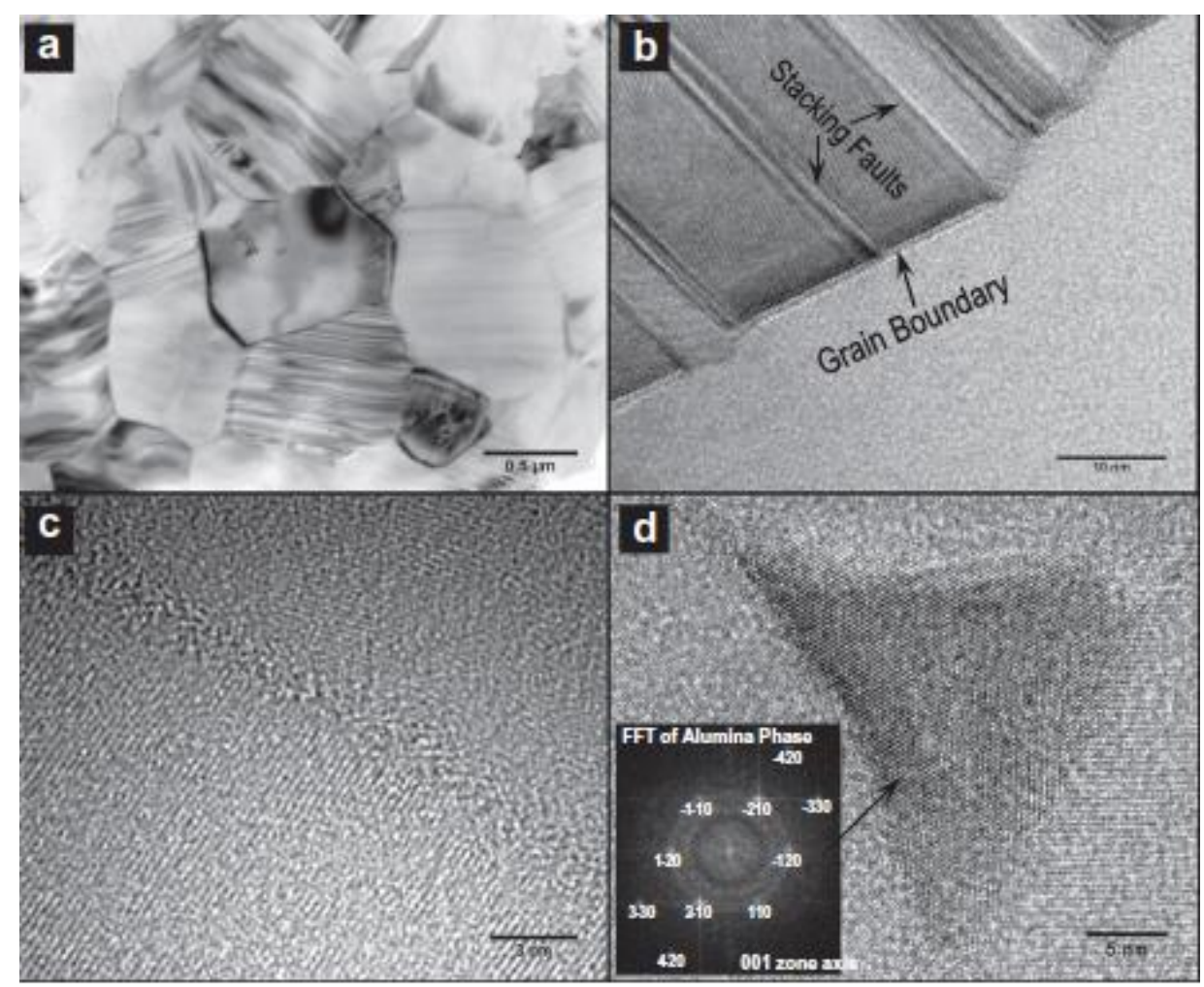

Fig. 50. (a) Bright field image of NITE SiC microstructure with $6 \mathrm{wt.} \%$ yttria-alumina, (b) phase contrast image of a grain boundary in NITE $\mathrm{SiC}$, (c) high-resolution image of the grain boundary between two silicon carbide grains filled with amorphous oxide, (d) phase contrast image of a triple-joint in NITE SiC filled with a crystalline alumina phase.

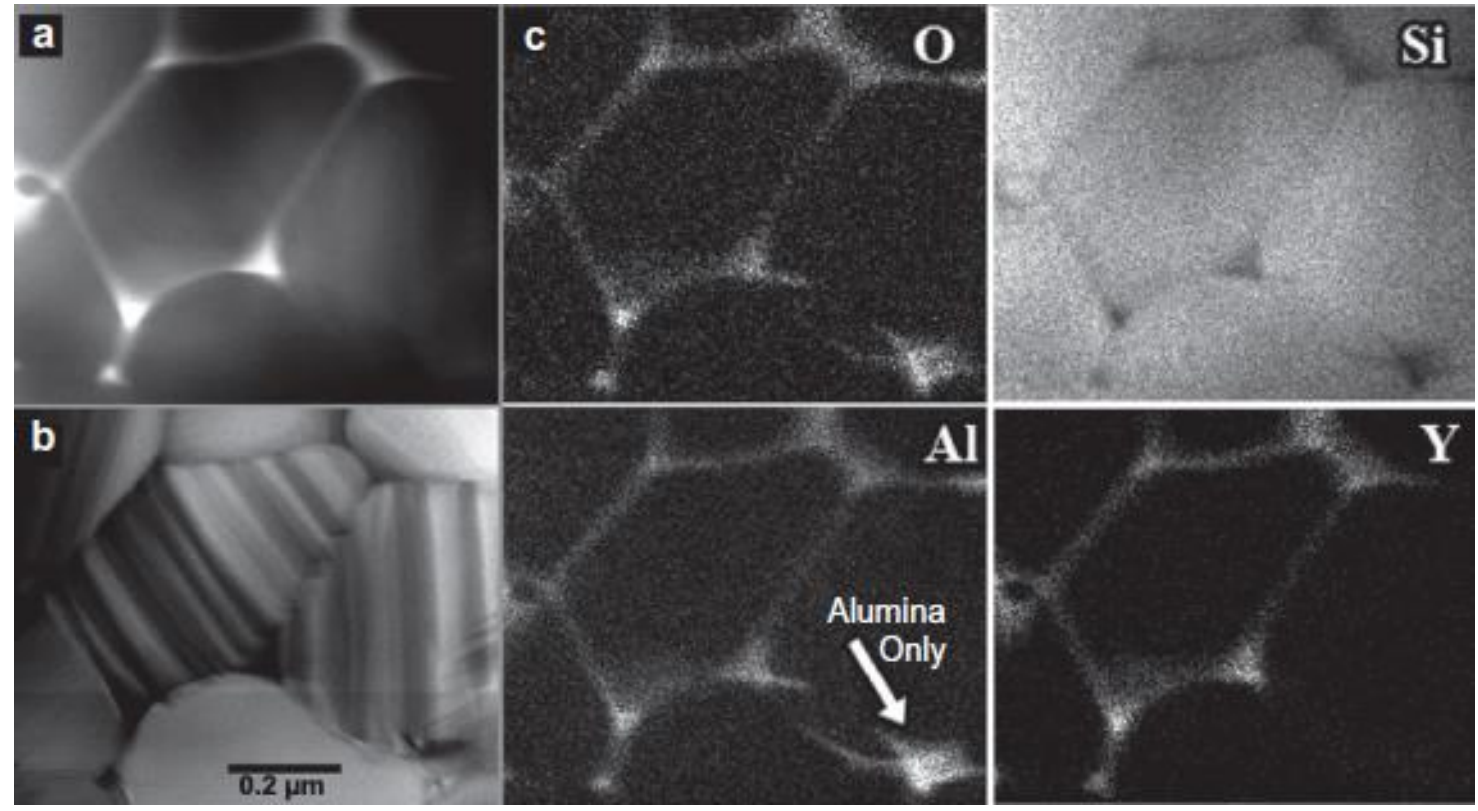

Fig. 51. (a) Z-contrast, (b) transmission images of NITE SiC microstructure in the scanning TEM mode, and (c) EDS map of oxide additives dispersed between $\mathrm{SiC}$ grains. 


\section{Inclusion of Poison}

A series was conducted to determine the effectiveness of alternative oxides to yttria, alumina, and silica which would also serve the required burnable poison function. Poisons that were chosen for study included gadolinium oxide, as shown in Figure 52. The results of the study indicated that matrix density and location of residual oxide were essentially unchanged up to a few percent poison inclusion.
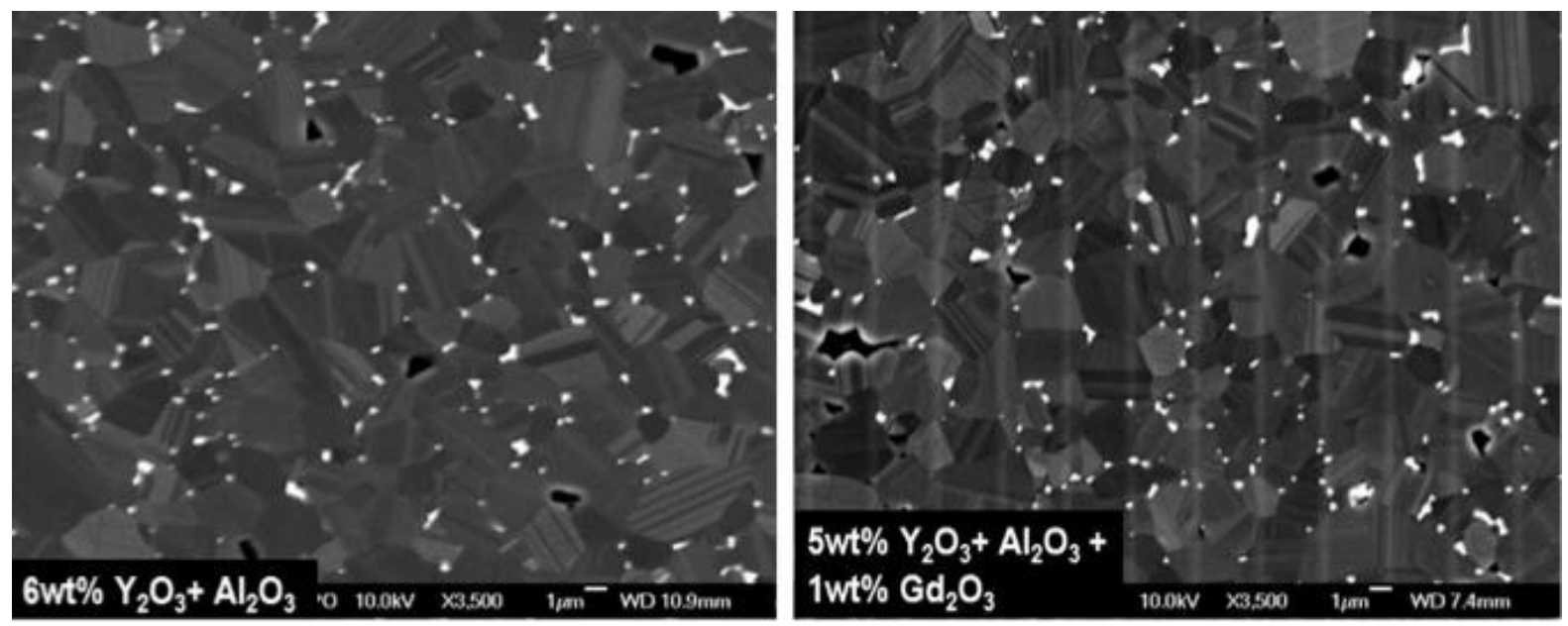

Fig. 52. Back-scattered SEM images of FCM fuel with standard $6 \% \mathrm{Y}_{2} \mathrm{O}_{3}$ sintering aid and $1 \% \mathrm{Gd}_{2} \mathrm{O}_{3}$ substitution.

\section{Development of UN Kernel: Modeling and Nitrogen Attack}

The preparation of UN by the carbothermic reduction of urania followed by nitriding, and the intimate contact of a UN TRISO kernel with the carbon of the buffer layer, is the reason for the current interest in the U-C-N system. The reported measurements with respect to U(C,N) were reviewed and used in a study to determine a solid solution representation of the phase. The model for the phase, together with free energies for the elemental and binary phases, was in turn used to derive high-temperature ternary U-C-N phase diagrams. Equilibrium nitrogen pressures have been computed for conditions of interest for fabrication and in-reactor behavior of U(C, N) LWR TRISO fuel.

The reported values of the free energy expression for $\mathrm{UN}$ all yield calculated nitrogen decomposition pressures that are significantly lower than almost all reported measurements. Figure 53 illustrates the disagreement between computed and measured values. This discrepancy also extends to measurements of nitrogen decomposition pressures measured over $\mathrm{U}(\mathrm{C}, \mathrm{N})$ when an ideal solution model is used to represent the phase, as detailed below. An effort was thus made to adjust the thermodynamic values for UN to obtain better agreement with nitrogen pressure data. (Note that considering the non-stoichiometry of UN would increase the discrepancy as hypostoichiometric UN would be computed to have an even lower nitrogen decomposition pressure.) The adjustment of the UN Gibbs free energy by $+12 \mathrm{~kJ} / \mathrm{mol}$ from the reported values yields an equivalent $298 \mathrm{~K}$ heat of formation of $-282.3526 \mathrm{~kJ} / \mathrm{mol}$, which brings the computed pressures into relative agreement with reported measurements (Figure 54), and the resultant free energy values for $\mathrm{UN}$ were used in representing $\mathrm{U}(\mathrm{C}, \mathrm{N})$. 
A model for the $\mathrm{U}(\mathrm{C}, \mathrm{N})$ phase was generated by assuming an ideal solution between the UC and UN phases. With the adjustment noted above, and concomitant adjustment to the free energy of the $\mathrm{U}_{2} \mathrm{~N}_{3}$ phase, it is possible to reproduce the phase diagram for the system as best as it is understood (example in Figure 54). The model also allows computing of nitrogen pressures as a function of temperature and composition within the various phase fields, an example of which is seen in Figure 55. The ability to provide predictive phase equilibria and chemical activities will be important in supporting fuel processing and in-reactor behavior.

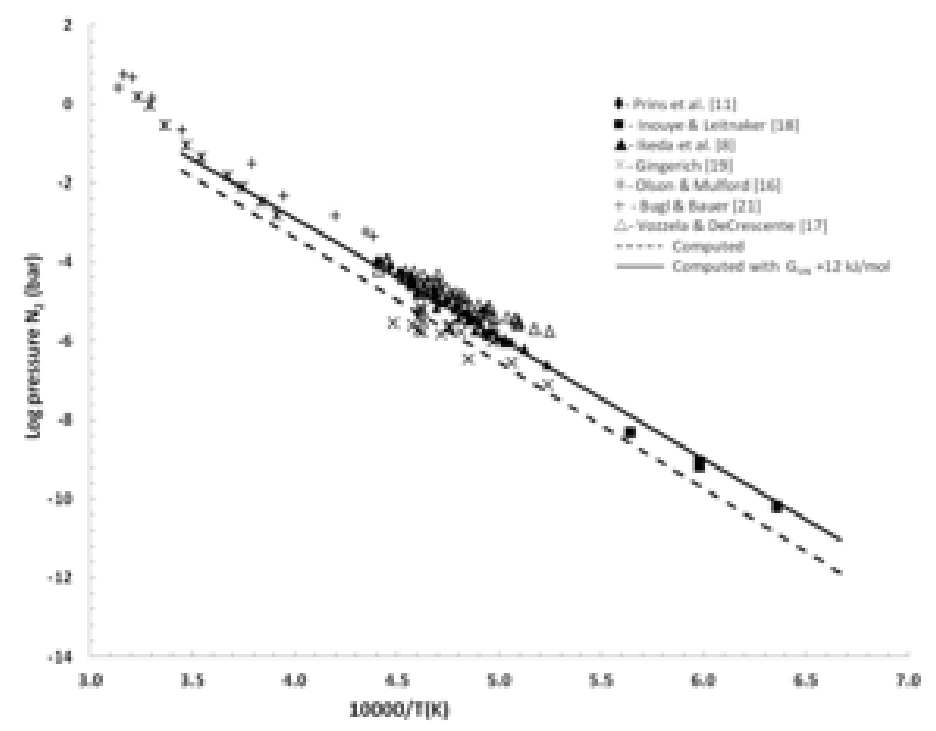

Fig. 53. Plot of reported UN decomposition nitrogen pressure reciprocal temperature data and computed pressures.

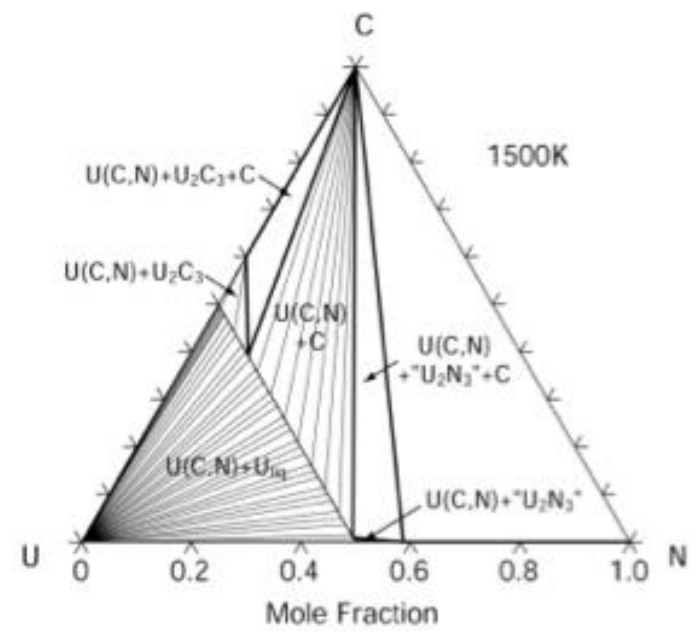

Fig. 54. Computed U-C-N phase diagram at $1500 \mathrm{~K}$ at 1 bar total. 


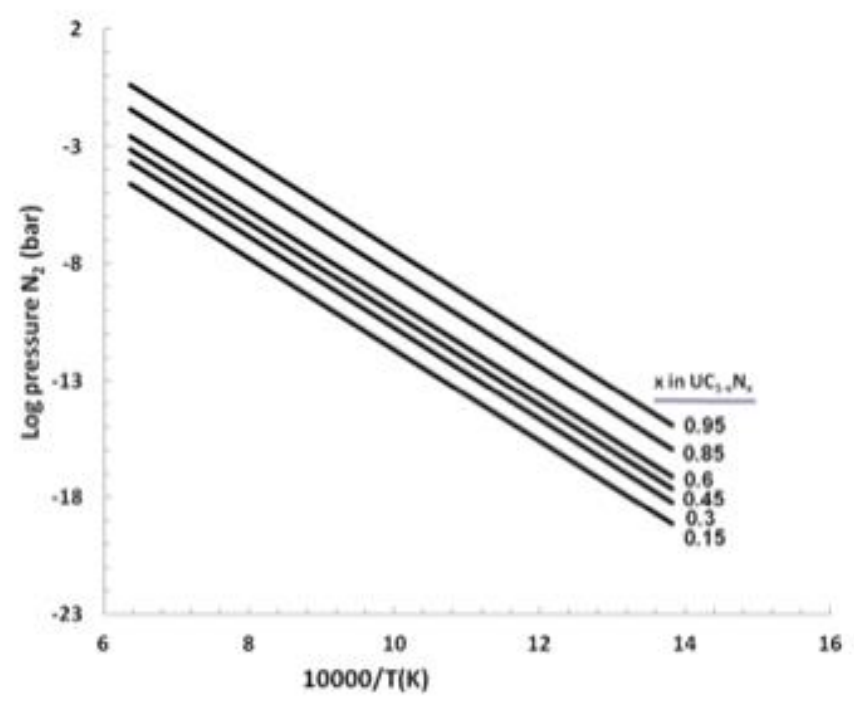

Fig. 55. Computed nitrogen pressure as a function of temperature over $723-1573 \mathrm{~K}$ and $\mathrm{UC}_{1-\mathrm{x}} \mathrm{N}_{\mathrm{x}}$ composition in equilibrium with carbon or carbides.

The use of UN fuel kernels for LWR TRISO fuel raises concern that the SiC layer may be nitrided by nitrogen from the fuel and thereby lose its effectiveness as a pressure and fission product barrier. Specifically, the concerns relate to free nitrogen produced during fission attacking the SiC layer. This would possibly jeopardize the stability of the $\mathrm{SiC}$ layer with the potential conversion into $\mathrm{Si}_{3} \mathrm{~N}_{4}$ or $\operatorname{Si}(\mathrm{C}, \mathrm{N})$.

The stability of the $\mathrm{SiC}$ layer in the presence of free nitrogen may be dependent upon the operating temperatures. Under accident scenarios, due to the high FCM thermal conductivity in comparison to $\mathrm{UO}_{2}$, the fuel temperature will be at most $100-200^{\circ} \mathrm{C}$ above the $1200^{\circ} \mathrm{C}$ LOCA condition, and then for only a limited period of time. Although nitrogen released in fissioning will form fission product nitrides, there will remain an overpressure of nitrogen of some magnitude. The nitrogen can be speculated to transport through the IPyC layer and contact the $\mathrm{SiC}$ layer. The $\mathrm{SiC}$ layer may be envisioned to fail due to resulting nitridation at the elevated temperatures. However, it is believed that these issues are particularly avoided in the LWR application. Lower temperatures will result in significantly lower nitrogen pressures. Lower temperatures will also substantially reduce nitrogen diffusion rates through the layers and nitriding kinetics. Kinetics calculations were performed using an expression for nitriding silicon. In order to further address these concerns, experiments were run with surrogate fuel particles under simulated operating conditions to determine the resulting phase formation at 700 and $1400^{\circ} \mathrm{C}$.

A review of the literature revealed very limited publications on nitriding of SiC. There is, however, considerable literature on nitriding of silicon, mostly for producing silicon nitride components. For purposes of assessing the issue of nitriding $\mathrm{SiC}$ in a TRISO particle, the use of relations for nitriding silicon would be a conservative assumption as elemental silicon would be more reactive. The intrinsic kinetics expression of Maalmi and Varma (Maalmi et al., 1996) for nitriding silicon powder was used to obtain a very conservative assessment and yielded Figure 56. 


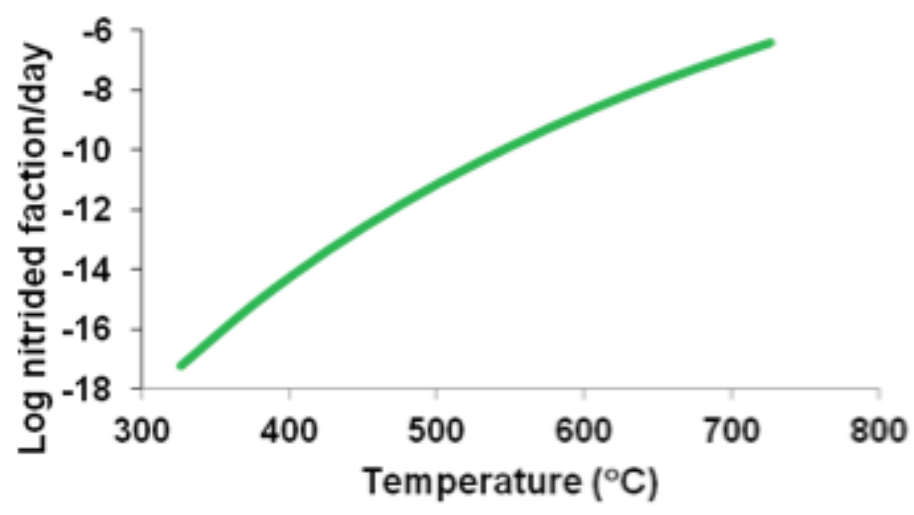

Fig. 56. Fraction of a 35 micron $\mathrm{SiC}$ layer nitrided per day in a reactor as a function of fuel temperature computed from the relations of Maalmi and Varma.

An experimental verification of the expected slow kinetics for nitriding SiC under LWR FCM conditions was performed using SiC-coated surrogate TRISO particles, that is, particles without the OPyC layer. These particles were exposed at $700^{\circ} \mathrm{C}$ in a $1 \mathrm{~atm}$. gas environment with flowing $60 \mathrm{ccm}$ argon with a balance of $4 \%$ hydrogen plus $240 \mathrm{ccm}$ of ultra-high-purity (UHP) nitrogen for $168 \mathrm{~h}$. Higher temperature exposure at $1400^{\circ} \mathrm{C}$ was made for $24 \mathrm{~h}$. An example of the results is shown in Figure 57.
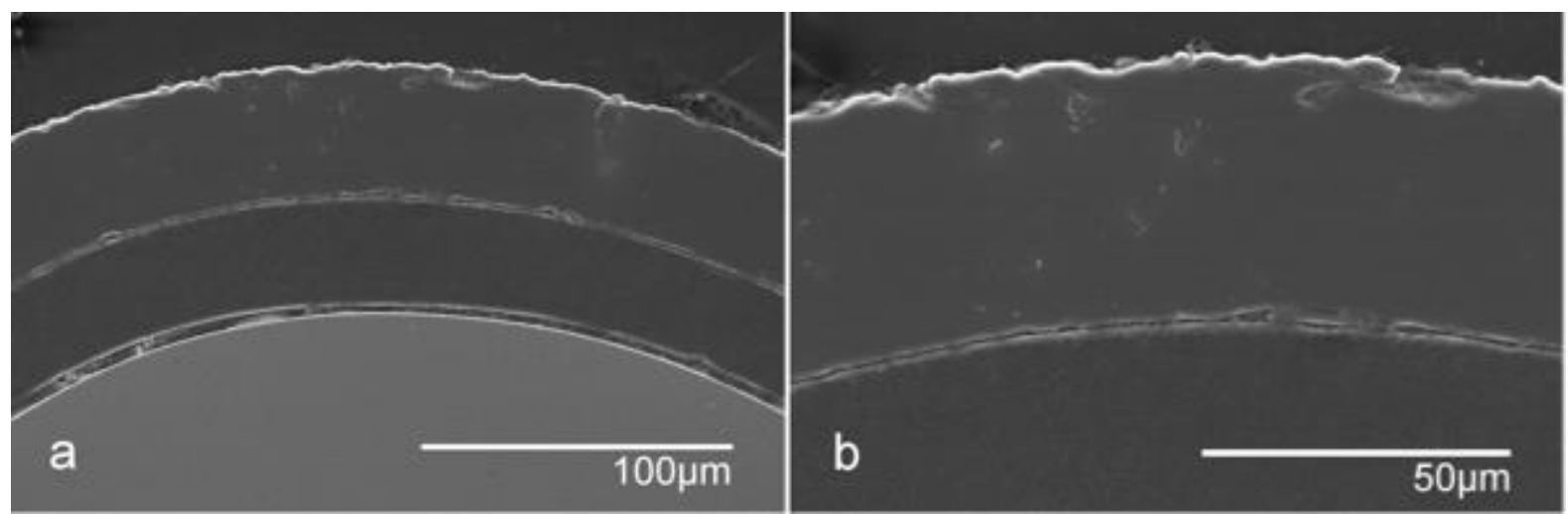

Fig. 57. Scanning electron microscopy images of $\mathrm{SiC}$ exposed to nitriding conditions for 24 hours.

Calculations based on very conservative assumptions and a set of experiments all indicate that under LWR FCM fuel operating conditions and limited period high-temperature accident conditions, the nitriding of the TRISO SiC layer is not an issue. The limited experimental results confirm this conclusion.

\section{Development of UN Kernel: Kernel Fabrication}

The goal for this work task is to understand and develop a process to produce high-density uranium carbonitride (UCN) kernels for use in an enhanced accident-tolerant fuel form in which the UCN kernels make up the core of TRISO coated particles that will be embedded in an FCM fuel form. The FY12 UCN kernel development began with the fabrication of uranium oxide microspheres using a well-developed internal gelation sol-gel process to successfully prepare uranium gel spheres with different concentrations of carbon black (Figure 58). 


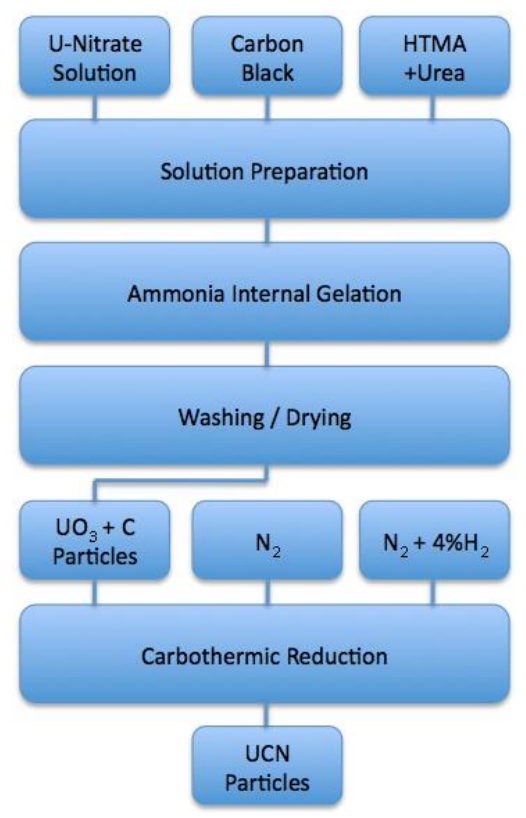

Fig. 58. UN kernel process flowsheet.

Homogeneous dispersion of carbon throughout the oxide microspheres is critical for efficient oxide-tonitride conversion, and an optimized process was established using a specific type of carbon and dispersing agent. Following the gelation step, the uranium oxide gel-spheres were washed and air dried before undergoing carbothermic reduction (CTR).

Considerable thermodynamic modeling experience in the uranium-carbon-nitrogen system was leveraged during the CTR phase of the R\&D. UCN compositions as a function of temperature and nitrogen pressure were calculated, and the relationships helped to guide development of a CTR process. In initial studies, $\mathrm{UO}_{3}+\mathrm{C}$ was investigated using a Simultaneous Thermal Analyzer coupled with a Quadrupole Mass Spectrometer. Kernels were thermally treated at $1400^{\circ} \mathrm{C}$ under flowing nitrogen gas. The sample weight loss, and corresponding evolution of oxygen and carbon, in the form of $\mathrm{CO}_{2}$ and $\mathrm{CO}$ gas, was recorded, and the time-to-conversion was determined. Kernels were then sintered to a density of approximately $13.4 \mathrm{~g} / \mathrm{cm}^{3}$ (93.7\% of theoretical density). The x-ray diffraction pattern shown in Figure 59 indicates a NaCl-type crystal structure, and comparison of the lattice parameter with data in the literature revealed the final composition to be $\mathrm{UC}_{0.16} \mathrm{~N}_{0.84}$. A process to fabricate uranium oxide microspheres with integral carbon and then to convert the microspheres to dense UCN kernels was successfully demonstrated, as shown in Figure 60. 


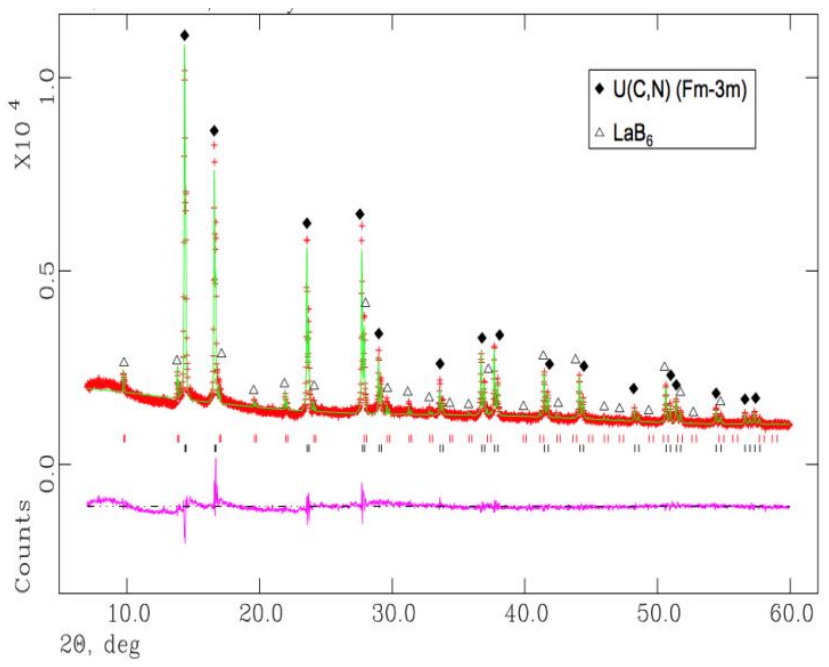

Fig. 59. X-ray diffraction pattern indicating single-phase UCN.

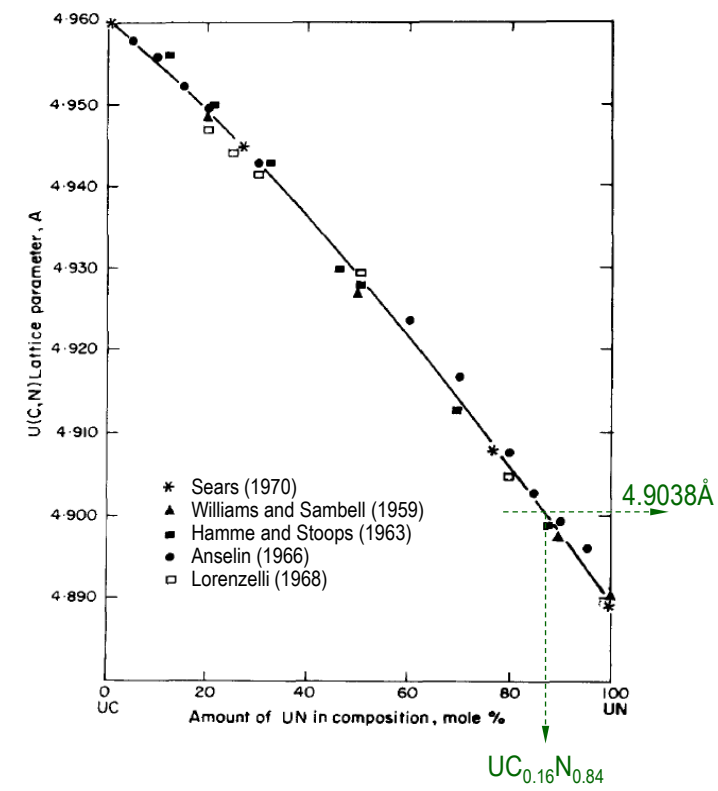

Fig. 60. Lattice parameter of UCN.

Subsequent R\&D has focused on scale-up to a process capable of fabricating larger quantities of kernels for TRISO coating development studies, and two methods are being evaluated: conversion in a fluidized bed and the traditional approach using a static bed of particles.

\subsubsection{Properties and Irradiation Test}

As discussed, the matrix of this fuel is based on the NITE formulation of $\mathrm{SiC}$ that was developed as an irradiation-stable SiC matrix for composites (Katoh et al., 2002). However, the applications and irradiation database for which $\mathrm{SiC}$ composites and NITE ceramics enjoy are primarily in the $600-1200^{\circ} \mathrm{C}$ range and relatively limited. The lower temperature (nominally $400-600^{\circ} \mathrm{C}$ ) projected for the LWR application is therefore of concern as the irradiation stability in this regime is unknown. For this reason, a 
series of surrogate FCM fuel pellets with TRISO fuel particles (zirconia substituted for the fissilecontaining kernel) were fabricated and irradiated in ORNL's HFIR to examine matrix irradiation of SiC. The pellets were clad in Zircaloy- 4 and irradiated at a target temperature of $325^{\circ} \mathrm{C} \pm 20$ to provide insight into any fuel-clad interactions. Irradiation temperature was determined for each capsule by using $\mathrm{SiC}$ temperature monitors following a post-irradiation protocol of isochronal annealing and thermal diffusivity measurement. Four capsules, each containing five pellets, were irradiated up to $\sim 8$ displacements per atom (dpa), or $\sim 8 \times 10^{25} \mathrm{n} / \mathrm{m}^{2}$ at $\mathrm{E}>0.1 \mathrm{MeV}$, at fluence intervals of $\sim 2 \mathrm{dpa}$. Of note is that all samples from this irradiation series reveal no apparent cracking, deformation, discoloration, or pellet clad interaction based on standard optical microscopy inspection of both surfaces. The extent of swelling of the pellets from the first two capsules is given in Figure 61, including samples for both surrogate containing samples (up to 39 vol\%) and pure NITE ceramic. Presented in Figure 61 is a broad data set of CVD SiC swelling (Snead et al., 2007) along with those of the FCM pellets, with and without TRISO. There was no correlation between swelling and surrogate TRISO loading, indicating that the swelling was dominated by the matrix. Also, from the figure it appears that the FCM fuel swells at a slightly higher rate (or to a greater extent) than that of CVD SiC, though is not far off the $\sim 300^{\circ} \mathrm{C} \mathrm{CVD} \mathrm{SiC} \mathrm{data.} \mathrm{It} \mathrm{is} \mathrm{noted} \mathrm{that}$ somewhat exaggerated swelling is inferred from the admittedly scarce data-set on NITE SiC swelling in the literature (Ozawa et al., 2009; Koyanagi et al., 2011).

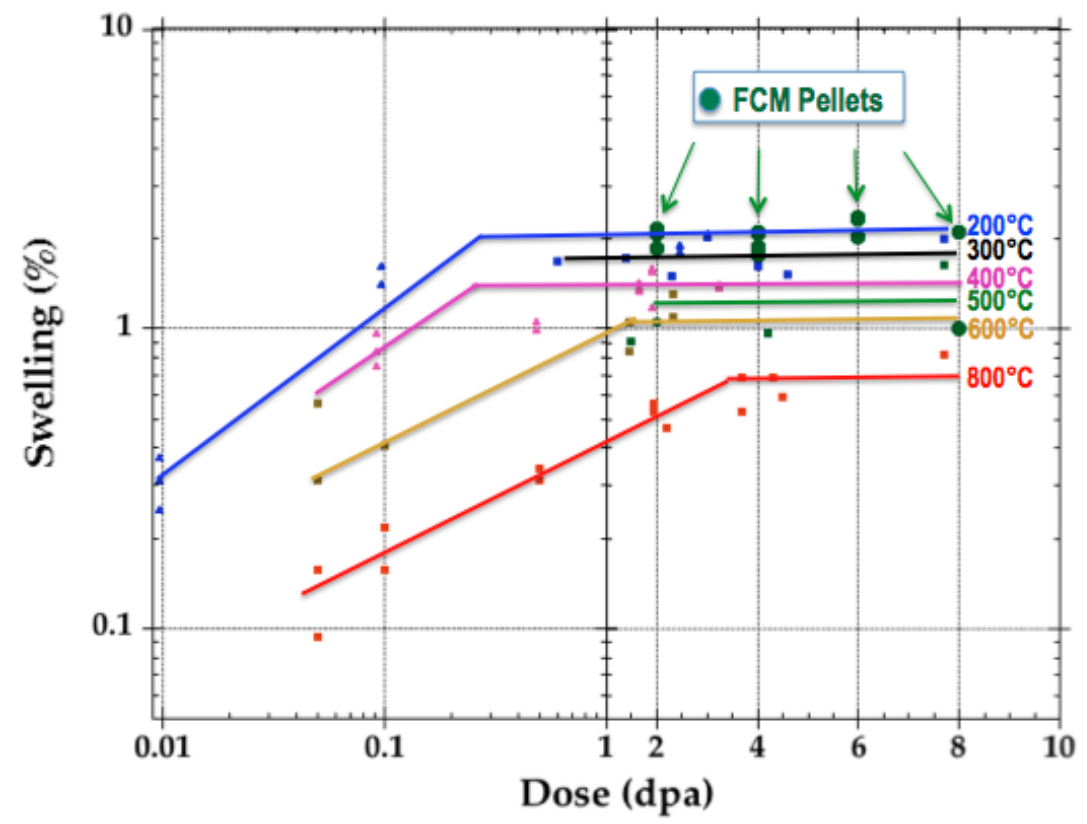

Fig. 61. Swelling data from recent HFIR irradiation of surrogate FCM pellets with a NITE SiC matrix compared to CVD SiC swelling data.

Figure 62 shows the saturation thermal conductivity of the irradiated FCM fuel compared with calculated values provided in reference (Terrani et al., 2012b). Values from this work are somewhat lower than previously calculated, but entirely consistent with the density-thermal conductivity correlation presented by Snead et al. (2007), suggesting behavior typical of SiC. Moreover, the thermal conductivity is seen to essentially saturate at the lowest dose of this study. 


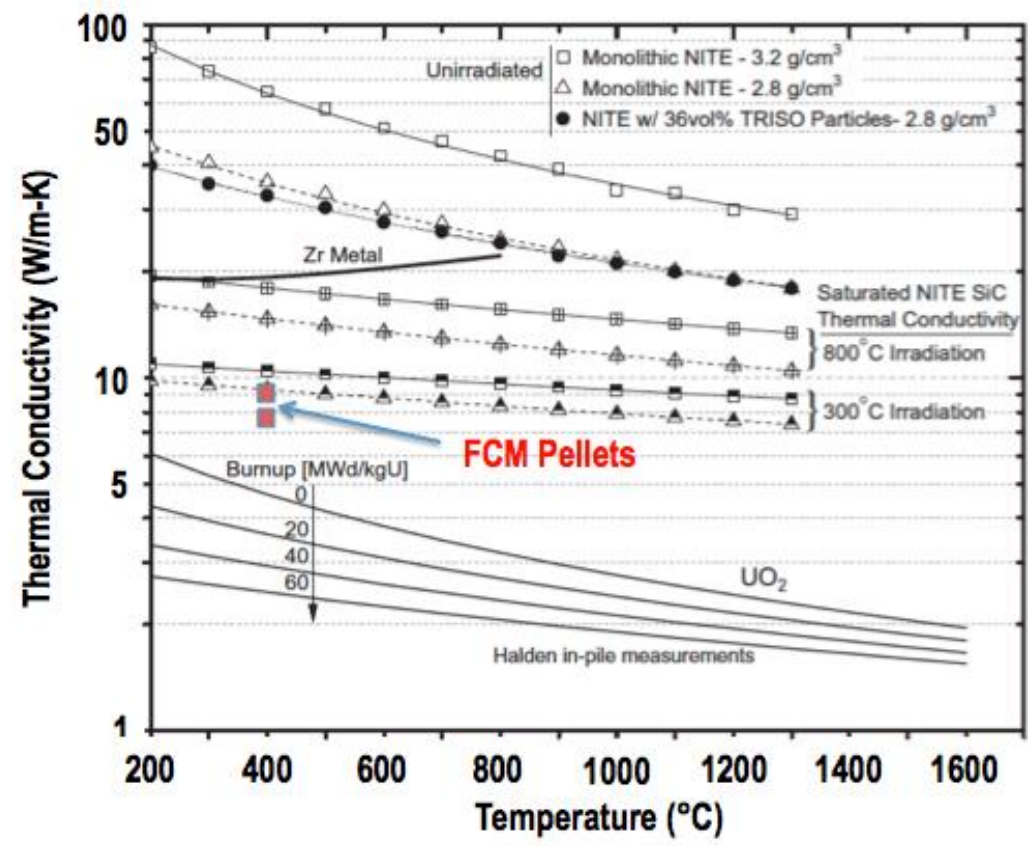

Fig. 62. Calculated, as-irradiated thermal conductivity of as a function of temperature of FCM fuel compared with measured thermal conductivity of $\mathrm{UO}_{2}$ and FCM.

\subsection{FUEL PERFORMANCE ASSESSMENT}

The structural components of the FCM fuel for the fuel performance analyses are the coated fuel particles (CFPs), the silicon carbide ( $\mathrm{SiC}$ ) pellet, the gap between the $\mathrm{SiC}$ pellet and cladding, and the cladding. The fuel performance analyses for the FCM fuel consist of

- estimation of the fuel burnup and depletion,

- analysis of the gas pressure buildup in the void volume (in kernel and buffer layer) in the CFPs,

- thermo-mechanical performance analysis for the CFPs,

- estimation of the failure fraction of a batch of CFPs,

- analysis of fission product transport from CFPs, through pellet and cladding, into the coolant,

- thermo-mechanical analysis on pellet and cladding and possible pellet-cladding interactions, and

- fuel arraying and rod dimensioning, and fuel rod thickness determination.

\subsubsection{Analytic Methods and Models}

The burnup and depletion data are determined from the detailed core analysis, in which the McCARD code is used. The thermo-mechanical analysis on the pellet and cladding of an FCM fuel rod will be estimated using commercial software like ABAQUS (ABAQUS, Inc., 1998).

The COPA code (Kim et al., 2008; IAEA, 2012) is used to analyze the fuel performance of the FCM fuel. The separate modules of COPA are now being integrated. The major functions of the individual COPA modules are the following.

1) Calculation of the neutron fluence, burnup, fission rate, power generation, and nuclide inventory throughout a fuel element and a CFP at a given location in the core. 
2) Calculation of the temperature distribution in a CFP, a pebble, and a unit cell of a prismatic block by a finite element method utilizing the Galerkin form of the weighted residuals procedure (Pepper and Heinrich, 1992). The numerical modeling is one-dimensional. For a CFP, the geometric elements for the numerical modeling are a kernel, a gap between a kernel and a buffer, a buffer, a gap between a buffer and an IPyC, an IPyC layer, a SiC layer, and an OPyC layer. For a pebble, the geometric elements are a fuel region and a matrix graphite region. The fuel region is assumed to be a mixture in which matrix graphite and CFPs are homogenously blended. For a unit cell, the geometric elements considered are a fuel compact, a graphite slab, and a gap between the compact and the graphite slab. The fuel compact is assumed to be a mixture in which matrix graphite and coated particles are homogeneously blended. The detailed mathematical modeling is described in a report (Kim, 2012).

3) Mechanical analyses of an intact or broken CFP by a finite element method using the Galerkin form of the weighted residuals procedure. The models are one-dimensional. The geometric elements for the numerical modeling are a fuel kernel, a buffer, and three coating layers. This calculates the contact forces or pressures acting on the interfaces between the layers of a CFP. The detailed mathematical modeling is described in the literature (Kim and Cho, 2009).

4) Calculation of the failure fraction of a batch of CFPs under reactor operation conditions, during irradiation experiments or heating tests. This function uses a Monte Carlo method for random particle sampling, in which a sample is equivalent to a CFP. The particle has different sizes, material properties, and mechanical strengths of the coating layers through the Monte Carlo sampling. The values for kernel diameter, the thickness of the buffer and of the three coating layers, and the density of kernel, buffer, and the three coating layers show the standard normal distribution. The strength of the silicon carbide and PyC layers is expressed as Weibull distributions.

5) Analysis of the fission product migration in a CFP, a pebble, and a fuel element under reactor operation conditions, irradiation experiments, or heating tests. The applied numerical scheme is a finite element method utilizing the Galerkin form of the weighted residual procedure. The detailed mathematical modeling is described in a report (Kim, 2012).

The capability of the COPA code has been validated using normal and accident benchmark problems of the International Atomic Energy Agency (IAEA) coordinated research program (CRP), IAEA CRP-6 (IAEA, 2012). The benchmark results of COPA are in good agreement with those of other benchmarked codes both for simplified analytical cases and for real irradiation experiments. Some deviations are due to the use of different physical-chemical models and different assumptions for input data or boundary conditions.

\subsubsection{Preliminary Scoping Analysis for OPR-1000}

\section{Coated Fuel Particle Analysis}

The CFP considered in this study is a TRISO-coated particle, which consists of a kernel, a low-density pyrocarbon layer (buffer), an IPyC layer, a SiC layer, and an OPyC layer. The kernel material is uranium mononitride (UN). Table 19 shows the average thicknesses and densities of the layers of the CFP. The thickness and density of the layers of a CFP are assumed to have a normal distribution, with 5\% and $1 \%$ standard deviations, respectively, used for the stochastic analysis of the failure fraction. 
Table 19. Thicknesses and densities of the CFP layers for the FCM-LWR

\begin{tabular}{lcc}
\hline Layers & Thickness $(\mu \mathrm{m})$ & Density $\left(\mathrm{g} / \mathrm{cm}^{3}\right)$ \\
\hline OPyC & $20 \pm 5 \%$ & $1.9 \pm 1 \%$ \\
$\mathrm{SiC}$ & $35 \pm 5 \%$ & $3.2 \pm 1 \%$ \\
$\mathrm{IPyC}$ & $35 \pm 5 \%$ & $1.9 \pm 1 \%$ \\
Buffer & $50 \pm 5 \%$ & $1.05 \pm 1 \%$ \\
Kernel & $350 \pm 5 \%$ & $14.32 \pm 1 \%$ \\
\hline
\end{tabular}

\section{Fuel Burnup and Depletion}

During the core analysis, McCARD was used to generate the burnup and depletion data for the FCM fuel. Figure 63 displays the relation between burnup and fast fluence as determined by McCARD. The final burnup and fast fluence are $60.5 \mathrm{GWd} / \mathrm{tHM}\left(6.2 \%\right.$ FIMA) and $10.7 \times 10^{21} \mathrm{n} / \mathrm{cm}^{2}(\mathrm{E}>0.18 \mathrm{MeV})$, respectively. The target lifetime is $1200 \mathrm{EFPD}$. The fast neutron fluence in the LWR FCM fuel is extended to more than $10 \times 10^{21} \mathrm{n} / \mathrm{cm}^{2}(\mathrm{E}>0.18 \mathrm{MeV})$, as compared to the usual 4 to $5 \times 10^{21} \mathrm{n} / \mathrm{cm}^{2}$ $(\mathrm{E}>0.18 \mathrm{MeV}$ ) typical of TRISO fuel in the HTR. The material properties related to the fuel performance analysis are presently known only within the range of the HTR fast neutron fluence. Lacking the relevant data, the current HTR materials properties should be only very carefully extrapolated into the extended fast neutron fluence to be expected for the FCM fuel in LWRs.

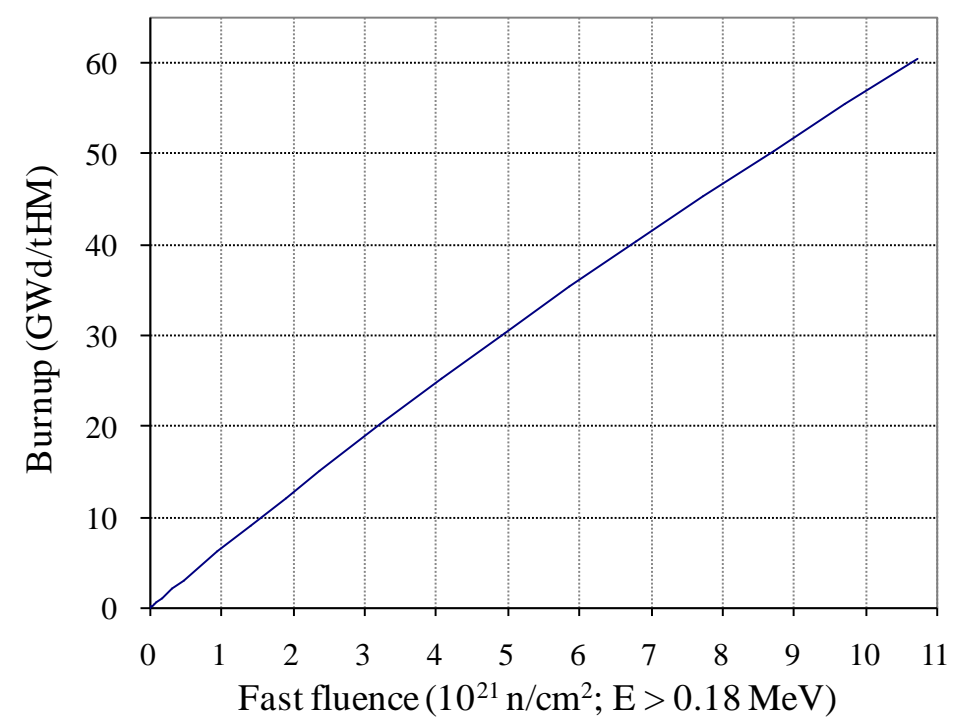

Fig. 63. Fuel burnup history.

\section{Gas Pressure Buildup in the Void Volume of Kernel and Buffer}

The generation of volatile species in the kernel is calculated in the form of fission yield with the HSC (Outotec, 2011) software and the McCARD code. The approximate expression obtained from the Booth model gives the release amount of gases into the void (open-pore) volume of the kernel and the buffer layer (Beck, 1960). The diffusivities of gases in the UN kernel are assumed to be same as for the $\mathrm{UO}_{2}$ kernel, which are described in an IAEA report (IAEA, 1997). Solid and gaseous swelling of the kernel occurs with burnup, and it causes the buffer to become denser, reducing its void volume. The gas pressure in the void volume can be estimated with the ideal gas law. 
Figures 64 to 66 show the gas pressure in the void volume at 600,850 , and $950^{\circ} \mathrm{C}$, respectively. At temperatures of 600 and $850^{\circ} \mathrm{C}$, xenon and krypton are major gas species. The total gas pressures are 0.012 and $0.13 \mathrm{MPa}$ at $10.7 \times 10^{21} \mathrm{n} / \mathrm{cm}^{2}(\mathrm{E}>0.18 \mathrm{MeV})$, respectively. At a temperature of $950^{\circ} \mathrm{C}$, cesium and cadmium become the major gas species. The total gas pressure is $3.88 \mathrm{MPa}$ at $10.7 \times 10^{21} \mathrm{n} / \mathrm{cm}^{2}$ $(\mathrm{E}>0.18 \mathrm{MeV})$. The estimation of gas pressure shows that cesium gas starts to be generated near $910^{\circ} \mathrm{C}$. The fuel temperature is likely to exceed $910^{\circ} \mathrm{C}$ at the center of the reactor core. The gas pressures are judged to be very low in all three cases, because no CO gas is generated in the UN kernel.

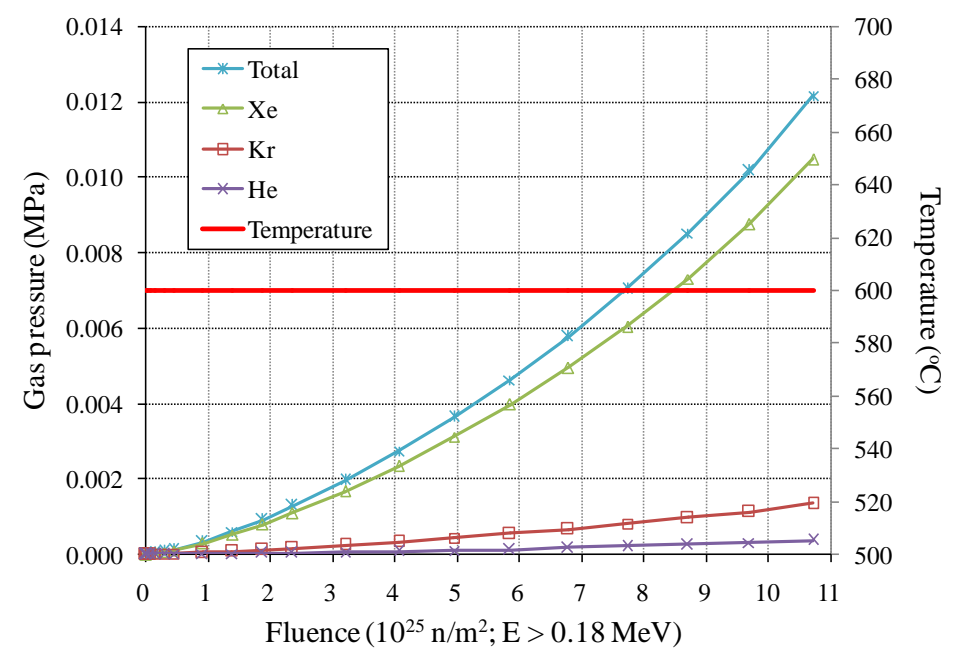

Fig. 64. Gas pressure in the void volume during normal operation at $600^{\circ} \mathrm{C}$.

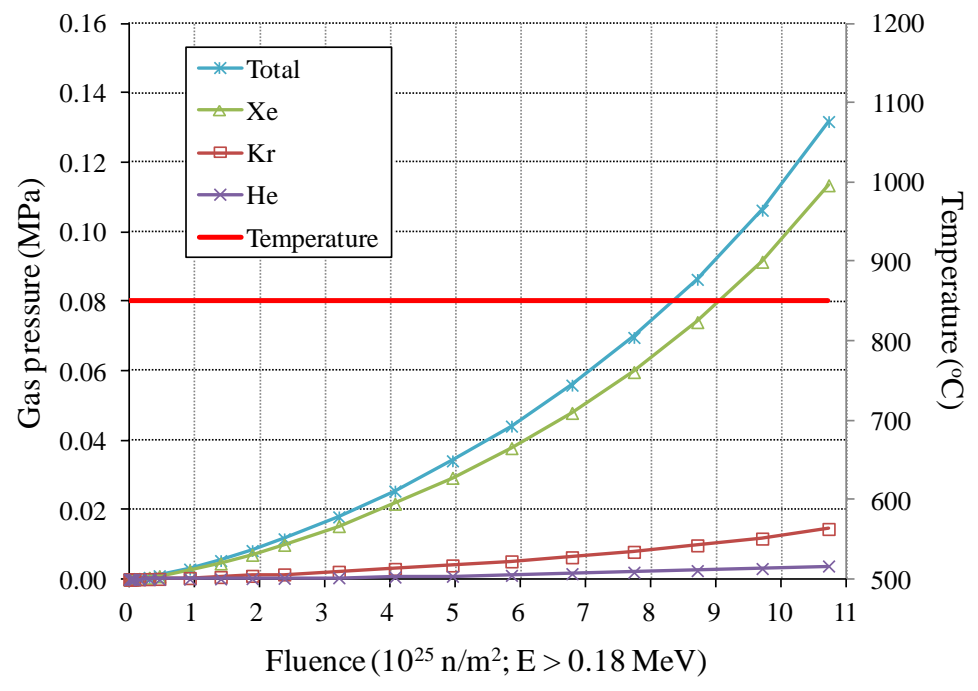

Fig. 65. Gas pressure in the void volume during normal operation at $850^{\circ} \mathrm{C}$. 


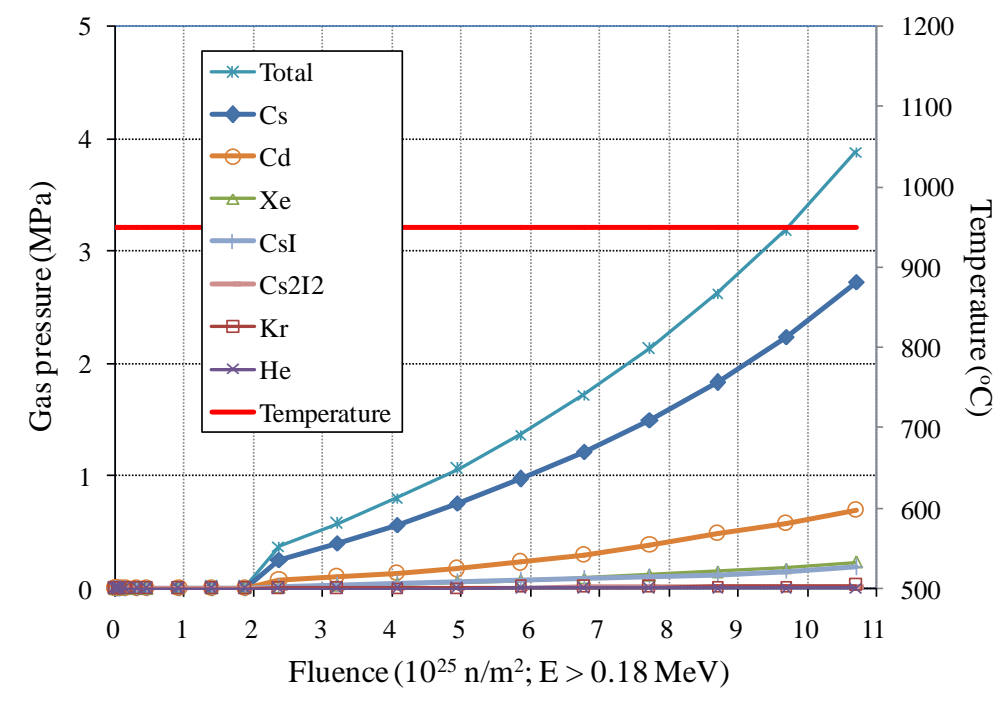

Fig. 66. Gas pressure in the void volume during normal operation at $950^{\circ} \mathrm{C}$.

\section{Thermo-mechanical Analysis of a Coated Fuel Particle}

Tables 20 to 23 show the properties of the TRISO materials utilized for the thermo-mechanical analysis. All the material properties are known below $4 \times 10^{21} \mathrm{n} / \mathrm{cm}^{2}(\mathrm{E}>0.18 \mathrm{MeV})$. Above $4 \times 10^{21} \mathrm{n} / \mathrm{cm}^{2}$ $(E>0.18 \mathrm{MeV})$, the material properties are assumed to have the same value as at $4 \times 10^{21} \mathrm{n} / \mathrm{cm}^{2}$ $(\mathrm{E}>0.18 \mathrm{MeV})$. A parabolic correlation for the irradiation-induced dimensional change (IIDC) of highdensity pyrocarbon has been proposed (DeMange et al., 2010) using data up to fluences of $4 \times 10^{21} \mathrm{n} / \mathrm{cm}^{2}$ $(\mathrm{E}>0.18 \mathrm{MeV})$, as shown in Figure 67. Two kinds of high-density pyrocarbon strength are considered: Weibull strength and mean fracture strength. The failure fractions using the two types of strengths are calculated and compared to each other. It is assumed that the irradiation does not affect the $\mathrm{SiC}$ strength (CEGA Corporation, 1993).

Figures 68 to 71 display the tangential stresses at the inner surfaces of the IPyC, SiC, and OPyC layers during irradiation. The IPyC is likely to break at the early stage of irradiation when the PyC strength is $200 \mathrm{MPa}$. The higher the temperature, the more severe will be the stress experienced by the SiC layer. In Figure 70, the tangential stresses of the SiC layer are nearly the same in both cases with and without gas pressure applied. This means that the major factor stressing the SiC layer is the IIDC of IPyC. The IPyC shrinks first, and then swells after about $5 \times 10^{21} \mathrm{n} / \mathrm{cm}^{2}(\mathrm{E}>0.18 \mathrm{MeV})$. The IPyC layer pushes the $\mathrm{SiC}$ layer out when it swells. When the $\mathrm{SiC}$ strength is $400 \mathrm{MPa}$, the $\mathrm{SiC}$ layer may break just after $9 \times 10^{21} \mathrm{n} / \mathrm{cm}^{2}(\mathrm{E}>0.18 \mathrm{MeV})$ at $600^{\circ} \mathrm{C}$, and it is highly likely to survive during the entire range of fluence at 850 and $950^{\circ} \mathrm{C}$. If the IPyC layer breaks at the early stage, the tangential stress acting on the $\mathrm{SiC}$ layer will largely decrease since the gas pressure is negligible. 
Table 20. Material properties of UN

\begin{tabular}{|c|c|c|}
\hline Parameters & Recommended correlations or values & Sources \\
\hline $\begin{array}{l}\text { Young's modulus, } \\
E(\mathrm{MPa})\end{array}$ & $\begin{array}{l}E=0.258 D^{3.002}\left(1-2.375 \times 10^{-5} T\right) \\
\text { where } D=\text { the fractional density }(\%) \in[70,100] \\
\quad T=\text { the temperature }(\mathrm{K}) \in[298,1473]\end{array}$ & (a) \\
\hline Poisson's ratio, $v$ & $\begin{array}{l}v=1.26 \times 10^{-3} D^{1.174} \\
\text { where } D=\text { the fractional density }(\%) \in[70,100]\end{array}$ & (a) \\
\hline $\begin{array}{l}\text { Steady-state creep rate, } \\
\dot{\varepsilon}^{c}\left(\mathrm{~s}^{-1}\right)\end{array}$ & $\begin{array}{l}\dot{\varepsilon}^{c}=2.054 \times 10^{-3} \sigma^{4.5} e^{-39369.5 / T} \\
\text { where } \sigma=\text { the stress }(\mathrm{MPa}) \in[20,34] \\
\quad T=\text { the temperature }(\mathrm{K}) \in[298,2523]\end{array}$ & (a) \\
\hline Swelling, $\frac{\Delta V}{V}(\%)$ & $\begin{array}{l}\frac{\Delta V}{V}=4.7 \times 10^{-11} T_{a v g}^{3.12} B^{0.83} D_{0}^{0.5} \\
\text { where } T_{a v g}=\text { the volume averaged fuel temperature }(\mathrm{K}) \\
\qquad B=\text { the fuel burnup (\%FIMA) } \\
D_{0}=\text { the as-fabricated fuel density (\%TD) }\end{array}$ & $(b)$ \\
\hline $\begin{array}{l}\text { Thermal expansion } \\
\text { coefficient, } \alpha\left(\mathrm{K}^{-1}\right)\end{array}$ & $\begin{array}{l}\alpha=7.096 \times 10^{-6}+1.409 \times 10^{-9} T \\
\text { where } T=\text { the temperature }(\mathrm{K})\end{array}$ & (c) \\
\hline $\begin{array}{l}\text { Thermal conductivity, } \\
k\left(\mathrm{~W} \mathrm{~m}^{-1} \mathrm{~K}^{-1}\right)\end{array}$ & $\begin{array}{l}k=1.864 \mathrm{e}^{-2.14 P} T^{0.361} \\
\text { where } P=\text { the porosity (dimensionless) } \in[0,0.2] \\
\quad T=\text { the temperature }(\mathrm{K}) \in[298,1923]\end{array}$ & $(d)$ \\
\hline
\end{tabular}

${ }^{a}$ Hayes, S.L., J.K. Thomas, and K.L. Peddicord. 1990a. "Material Property Correlations for Uranium Mononitride II. Mechanical properties," Journal of Nuclear Materials, 171, 271-288.

${ }^{b}$ Ross, S.B. and M.S. El-Genk. 1990. “Uranium Nitride Fuel Swelling Correlation,” Journal of Nuclear Materials, 170, 169-177.

${ }^{c}$ Hayes, S.L., J.K. Thomas, and K.L. Peddicord. 1990b. "Material Property Correlations for Uranium Mononitride I. Physical properties," Journal of Nuclear Materials, 171, 262-270.

${ }^{d}$ Hayes, S.L., J.K. Thomas, and K.L. Peddicord. 1990c. "Material Property Correlations for Uranium Mononitride III. Transport properties," Journal of Nuclear Materials, 171, 289-299. 
Table 21. Material properties of low-density pyrocarbon

\begin{tabular}{|c|c|c|}
\hline Parameters & Recommended correlations or values & Sources \\
\hline $\begin{array}{l}\text { Young's modulus, } \\
E(\mathrm{MPa})\end{array}$ & $\begin{array}{l}E=34475 \mathrm{e}^{-2.03 P} E(\varphi) E(T) \\
\text { where } P=\text { porosity }(-) \in[0.2,0.56] \\
E(\varphi)=1+0.23 \varphi \\
E(T)=1+0.00015(T-20) \\
\varphi=\text { fast neutron fluence } \\
\quad\left(10^{21} \mathrm{n} / \mathrm{cm}^{2} ; \mathrm{E}>0.18 \mathrm{MeV}\right) \in[0,4] \\
T=\text { temperature }\left({ }^{\circ} \mathrm{C}\right) \in[20,2000]\end{array}$ & $(a),(b)$ \\
\hline Poisson's ratio, $v$ & 0.23 & (b) \\
\hline $\begin{array}{l}\text { Irradiation-induced } \\
\text { creep coefficient, } \\
K\left(10^{21} \mathrm{n} / \mathrm{cm}^{2} ; \mathrm{E}>0.18\right. \\
\mathrm{MeV})\end{array}$ & $\begin{array}{l}K=\left(2.193 \times 10^{-4}-4.85 \times 10^{-7} T+4.0147 \times 10^{-10} T^{2}\right) \\
\quad \cdot[1+2.38(1.9-\rho)] \\
\text { where } T=\text { temperature }\left({ }^{\circ} \mathrm{C}\right) \\
\quad \rho=\text { density }\left(\mathrm{g} / \mathrm{cm}^{3}\right)<2.05\end{array}$ & $(a),(b)$ \\
\hline $\begin{array}{l}\text { Poisson's ratio in creep, } \\
\mu\end{array}$ & 0.5 & (a) $(c)$ \\
\hline $\begin{array}{l}\text { Irradiation-induced } \\
\text { dimensional change, } \\
S(\%)\end{array}$ & $\begin{array}{l}S=(0.176 / 1.75)\left(\mathrm{e}^{-1.75 \varphi}-1\right) \\
\text { where } \varphi=\text { fast neutron fluence } \\
\qquad\left(10^{21} \mathrm{n} / \mathrm{cm}^{2} ; \mathrm{E}>0.1 \mathrm{MeV}\right)\end{array}$ & (c) \\
\hline $\begin{array}{l}\text { Thermal expansion } \\
\text { coefficient, } \alpha\left(10^{-6} \mathrm{~K}^{-1}\right)\end{array}$ & 3.5 & (c) \\
\hline $\begin{array}{l}\text { Thermal conductivity, } \\
k\left(\mathrm{~W} \mathrm{~m}^{-1} \mathrm{~K}^{-1}\right)\end{array}$ & 0.5 & $(c)$ \\
\hline Weibull modulus, $\mathrm{m}$ & 3 & $(a)$ \\
\hline $\begin{array}{l}\text { Weibull strength, } \\
\sigma_{m s}(\mathrm{MPa})\end{array}$ & 35 & (a) \\
\hline
\end{tabular}

${ }^{a}$ CEGA Corporation. 1993. NP-MHTGR Material Models of Pyrocarbon and Pyrolytic Silicon Carbide, CEGA-002820, Rev. 1.

${ }^{b}$ Miller, G.K., D.A. Petti, J.T. Maki, and D.L. Knudson. 2009. PARFUME Theory and Model Basis, Report INL/EXT-08-14497.

${ }^{c}$ INEEL, CEA, MIT, 2004. Development of Improved Models and Designs for Coated-Particle Gas Reactor Fuels, INEEL/EXT-05-02615, Idaho National Engineering and Environmental Laboratory. 
Table 22. Material properties of high-density pyrocarbon

\begin{tabular}{|c|c|c|c|c|c|c|}
\hline Parameters & \multicolumn{5}{|c|}{ Recommended correlations or values } & Sources \\
\hline $\begin{array}{l}\text { Young's modulus, } \\
E(\mathrm{MPa})\end{array}$ & \multicolumn{5}{|c|}{$\begin{array}{l}E_{i}=E_{i 0} E(\rho) E_{i}\left(\mathrm{BAF}_{0}\right) E\left(\mathrm{~L}_{\mathrm{c}}\right) E(\varphi) E(T) \\
\text { where } i=r \text { (radial direction), } \theta \text { (tangential direction) } \\
E_{r 0}=E_{\theta 0}=25.5 \mathrm{MPa} \\
\quad E(\rho)=0.384+0.324 \rho \\
E_{\theta}\left(\mathrm{BAF}_{0}\right)=0.481+0.519 \mathrm{BAF}_{0} \\
E_{r}\left(\mathrm{BAF}_{0}\right)=1.463-0.463 \mathrm{BAF}_{0} \\
E\left(\mathrm{~L}_{\mathrm{c}}\right)=2.985-0.0662 \mathrm{~L}_{\mathrm{c}} \\
E(\varphi)=1+0.23 \varphi \\
E(T)=1+0.00015(T-20) \\
\rho=\text { the density }\left(\mathrm{g} / \mathrm{cm}^{3}\right) \in[1.8,2.2] \\
\quad \mathrm{BAF} F_{0}=\text { the initial Bacon anisotropy factor } \in[1,1.25] \\
\quad \mathrm{L}_{\mathrm{c}}=\text { the apparent crystallite size }(\AA) \in[25,35] \\
\quad \varphi=\text { fast neutron fluence } \\
\quad\left(10^{21} \mathrm{n} / \mathrm{cm}^{2} ; \mathrm{E}>0.18 \mathrm{MeV}\right) \in[0,4] \\
T=\text { the temperature }\left({ }^{\circ} \mathrm{C}\right) \in[20,2000]\end{array}$} & (a) \\
\hline Poisson's ratio, $v$ & \multicolumn{5}{|c|}{0.23} & (b) \\
\hline $\begin{array}{l}\text { Irradiation-induced } \\
\text { creep coefficient, } \\
K\left(10^{21} \mathrm{n} / \mathrm{cm}^{2} ; \mathrm{E}>0.18 \mathrm{MeV}\right)\end{array}$ & \multicolumn{5}{|c|}{$\begin{array}{l}K=\left(2.193 \times 10^{-4}-4.85 \times 10^{-7} T+4.0147 \times 10^{-10} T^{2}\right) \\
\quad \cdot[1+2.38(1.9-\rho)] \\
\text { where } T=\text { temperature }\left({ }^{\circ} \mathrm{C}\right) \\
\quad \rho=\text { density }\left(\mathrm{g} / \mathrm{cm}^{3}\right)<2.05\end{array}$} & (a) \\
\hline Poisson's ratio in creep, $\mu$ & \multicolumn{5}{|c|}{0.5} & $(a)(c)$ \\
\hline \multirow{5}{*}{$\begin{array}{l}\text { Irradiation-induced } \\
\text { dimensional change, } \\
S_{r}, S_{\theta}(\%)\end{array}$} & \multicolumn{5}{|c|}{$\begin{array}{l}S_{i}=a_{i} \varphi+b_{i} \varphi^{2} \\
\text { where } i=r \text { (radial direction), } \theta \text { (tangential direction) } \\
\quad \varphi=\text { fast neutron fluence }\left(10^{21} \mathrm{n} / \mathrm{cm}^{2} ; \mathrm{E}>0.18 \mathrm{MeV}\right)\end{array}$} & \multirow{5}{*}{$(d)$} \\
\hline & $T\left({ }^{\circ} \mathrm{C}\right)$ & $a_{r}$ & $b_{r}$ & $a_{\theta}$ & $b_{\theta}$ & \\
\hline & 600 & -1.044 & 0.153 & -1.276 & 0.141 & \\
\hline & 1050 & -1.145 & 0.280 & -1.493 & 1.139 & \\
\hline & \multicolumn{5}{|c|}{$\begin{array}{l}\text { Note. The values in the above table are obtained at the density of } 1.85 \\
\mathrm{~g} / \mathrm{cm}^{3} \text { and the initial BAF of } 1.036 \text {. }\end{array}$} & \\
\hline $\begin{array}{l}\text { Thermal expansion } \\
\text { coefficient, } \alpha\left(10^{-6}{ }^{\circ} \mathrm{C}^{-1}\right)\end{array}$ & \multicolumn{5}{|c|}{$\begin{array}{l}\alpha_{r}=\mathrm{A}\left(-37.5 R_{r}+30\right), \alpha_{\theta}=\mathrm{A}\left[36\left(R_{\theta}-1\right)^{2}+1\right] \\
\text { where } R_{r}=2 /(2+\mathrm{BAF}), R_{\theta}=1-R_{r} / 2, \\
\quad \mathrm{~A}=1+0.11(T-400) / 700 \\
\quad T=\text { temperature }\left({ }^{\circ} \mathrm{C}\right) \\
\quad \mathrm{BAF}=\text { Bacon anisotropy factor }\end{array}$} & (b) \\
\hline $\begin{array}{l}\text { Thermal conductivity, } \\
k\left(\mathrm{~W} \mathrm{~m}^{-1} \mathrm{~K}^{-1}\right)\end{array}$ & \multicolumn{5}{|c|}{4} & $(c)$ \\
\hline Strength & \multirow{2}{*}{\multicolumn{5}{|c|}{$\begin{array}{l}\text { Weibull modulus, } m \text {, and Weibull strength, } \sigma_{m s}(\mathrm{MPa}) \text { : } \\
m=10.1-0.587 \mathrm{BAF}_{0} \\
\sigma_{m s}=\left(2918.7 \mathrm{BAF}_{0}^{2}-2666 \mathrm{BAF}_{0}\right)[E(\varphi) E(T)]^{1 / 2} \\
\text { where } \mathrm{BAF}_{0}=\text { initial Bacon anisotropy factor } \\
\quad E(\varphi)=1+0.23 \varphi \\
\quad E(T)=1+0.00015(T-20) \\
\quad \varphi=\text { fast neutron fluence } \\
\quad\left(10^{21} \mathrm{n} / \mathrm{cm}^{2} ; \mathrm{E}>0.18 \mathrm{MeV}\right) \in[0,4 \\
T=\text { temperature }\left({ }^{\circ} \mathrm{C}\right) \in[20,2000] \\
\text { Mean fracture strength }=200 \mathrm{MPa}\end{array}$}} & (a) \\
\hline & & & & & & $(d)$ \\
\hline
\end{tabular}

${ }^{a}$ CEGA Corporation, 1993. NP-MHTGR Material Models of Pyrocarbon and Pyrolytic Silicon Carbide, CEGA-002820, Rev. 1 .

${ }^{b}$ Miller, G.K., D.A. Petti, J.T. Maki, and D.L. Knudson. 2009. PARFUME Theory and Model Basis Report, INL/EXT-0814497.

${ }^{c}$ INEEL, CEA, MIT, 2004. Development of Improved Models and Designs for Coated-Particle Gas Reactor Fuels, Idaho National Engineering and Environmental Laboratory, INEEL/EXT-05-02615.

${ }^{d}$ DeMange, P., J. Marian, M. Caro, M., and A. Caro. 2010. "TRISO-fuel Element Thermo-mechanical Performance Modeling for the Hybrid LIFE Engine with Pu Fuel Blanket," Journal of Nuclear Materials, 405, 144-155. 
Table 23. Material properties of silicon carbide

\begin{tabular}{|c|c|c|}
\hline Parameters & Recommended correlations or values & Sources \\
\hline $\begin{array}{l}\text { Young's modulus, } \\
E(\mathrm{MPa})\end{array}$ & $\begin{array}{l}E=\left(432 \times 10^{3}-0.0741 \times 10^{3} T+1.541 \times 10^{-1} T^{2}-5.401 \times 10^{-4} T^{3}\right. \\
\left.+8.142 \times 10^{-7} T^{4}-5.18 \times 10^{-10} T^{5}+1.043 \times 10^{-13} T^{6}\right) \cdot \mathrm{e}^{-3.12 P} \\
\text { where } T=\text { temperature }\left({ }^{\circ} \mathrm{C}\right), P=\text { the porosity }(-)\end{array}$ & (a) \\
\hline Poisson's ratio, $v$ & 0.13 & $(a)$ \\
\hline $\begin{array}{l}\text { Thermal expansion } \\
\text { coefficient, } \alpha\left(\mathrm{K}^{-1}\right)\end{array}$ & $\begin{array}{l}\alpha=3.43846 \times 10^{-6}+1.19402 \times 10^{-9} T-2.05716 \times 10^{-13} T^{2} \\
\text { where } T=\text { temperature }\left({ }^{\circ} \mathrm{C}\right)\end{array}$ & (a) \\
\hline $\begin{array}{l}\text { Thermal conductivity, } \\
k\left(\mathrm{~W} \mathrm{~m}^{-1} \mathrm{~K}^{-1}\right)\end{array}$ & 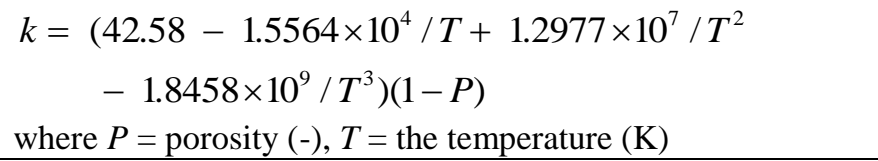 & (a) \\
\hline \multirow[t]{2}{*}{ Strength } & $\begin{array}{l}\text { Weibull modulus, } m \text {, and Weibull strength, } \sigma_{m s}(\mathrm{MPa}) \text { : } \\
m=6, \sigma_{m s}=770\end{array}$ & $(b),(c)$ \\
\hline & Mean fracture strength $=400 \mathrm{MPa}$ & (d) \\
\hline
\end{tabular}

${ }^{a}$ INEEL, CEA, MIT. 2004. Development of Improved Models and Designs for Coated-Particle Gas Reactor Fuels, INEEL/EXT-05-02615, Idaho National Engineering and Environmental Laboratory.

${ }^{b}$ CEGA Corporation. 1993. NP-MHTGR Material Models of Pyrocarbon and Pyrolytic Silicon Carbide, CEGA002820, Rev. 1.

${ }^{c}$ Miller, G.K., D.A. Petti, J.T. Maki, and D.L. Knudson. 2009. PARFUME Theory and Model Basis Report, INL/EXT-08-14497.

${ }^{d}$ DeMange, P., J. Marian, M. Caro, and A. Caro. 2010. "TRISO-fuel Element Thermo-mechanical Performance Modeling for the Hybrid LIFE Engine with Pu Fuel Blanket.," Journal of Nuclear Materials, 405, 144-155. 


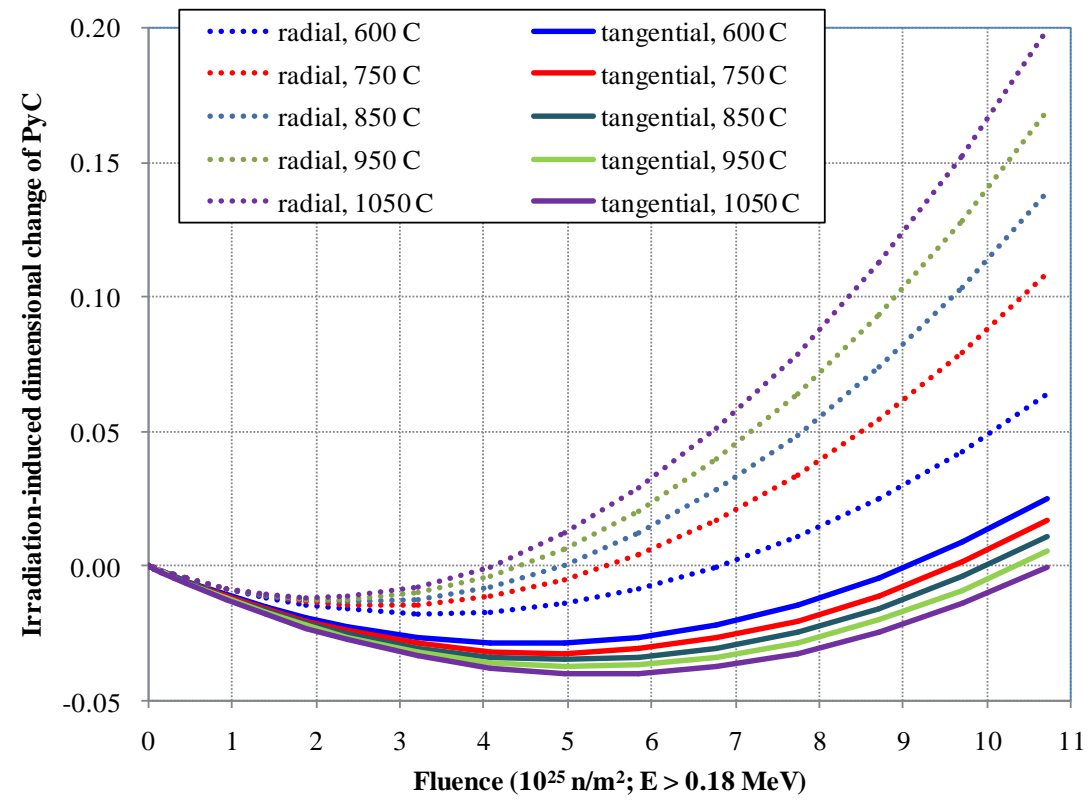

Fig. 67. Irradiation-induced dimensional change of high-density pyrocarbon.

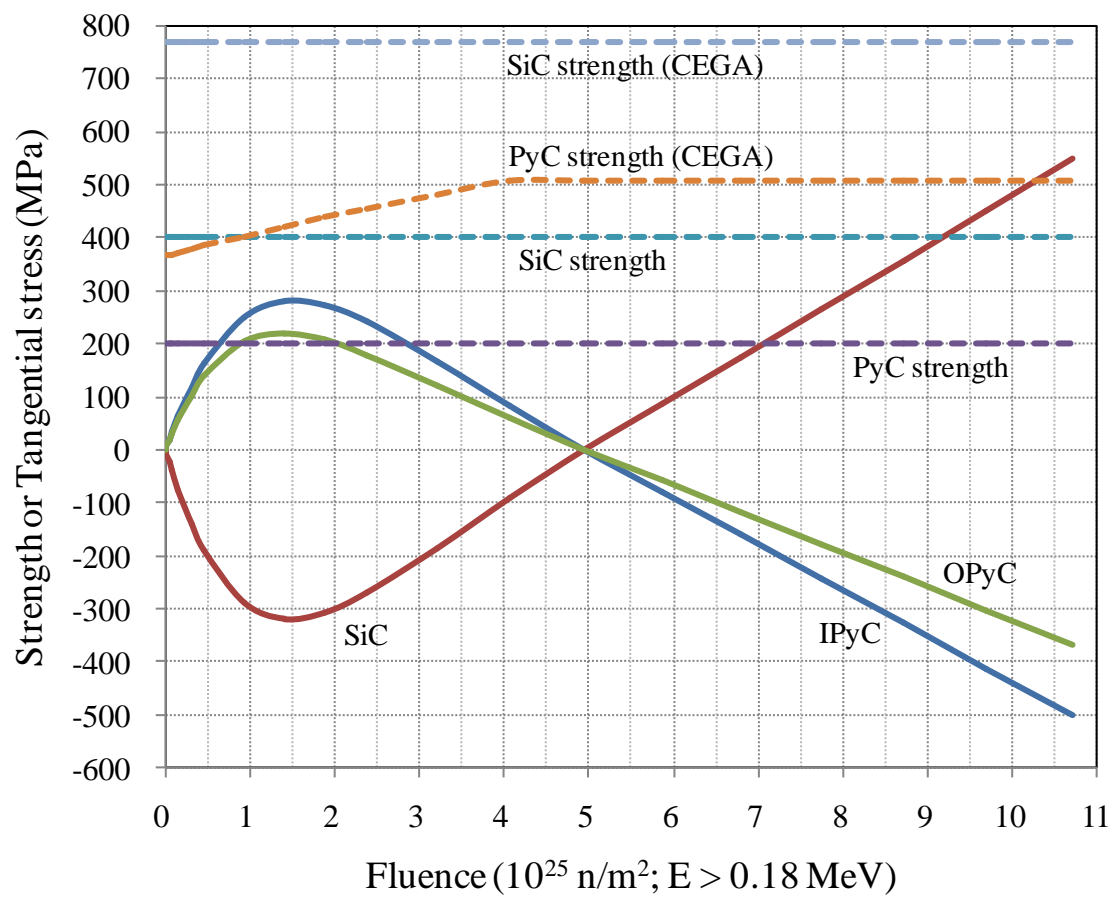

Fig. 68. Stress evolutions during normal operation at $600^{\circ} \mathrm{C}$. 


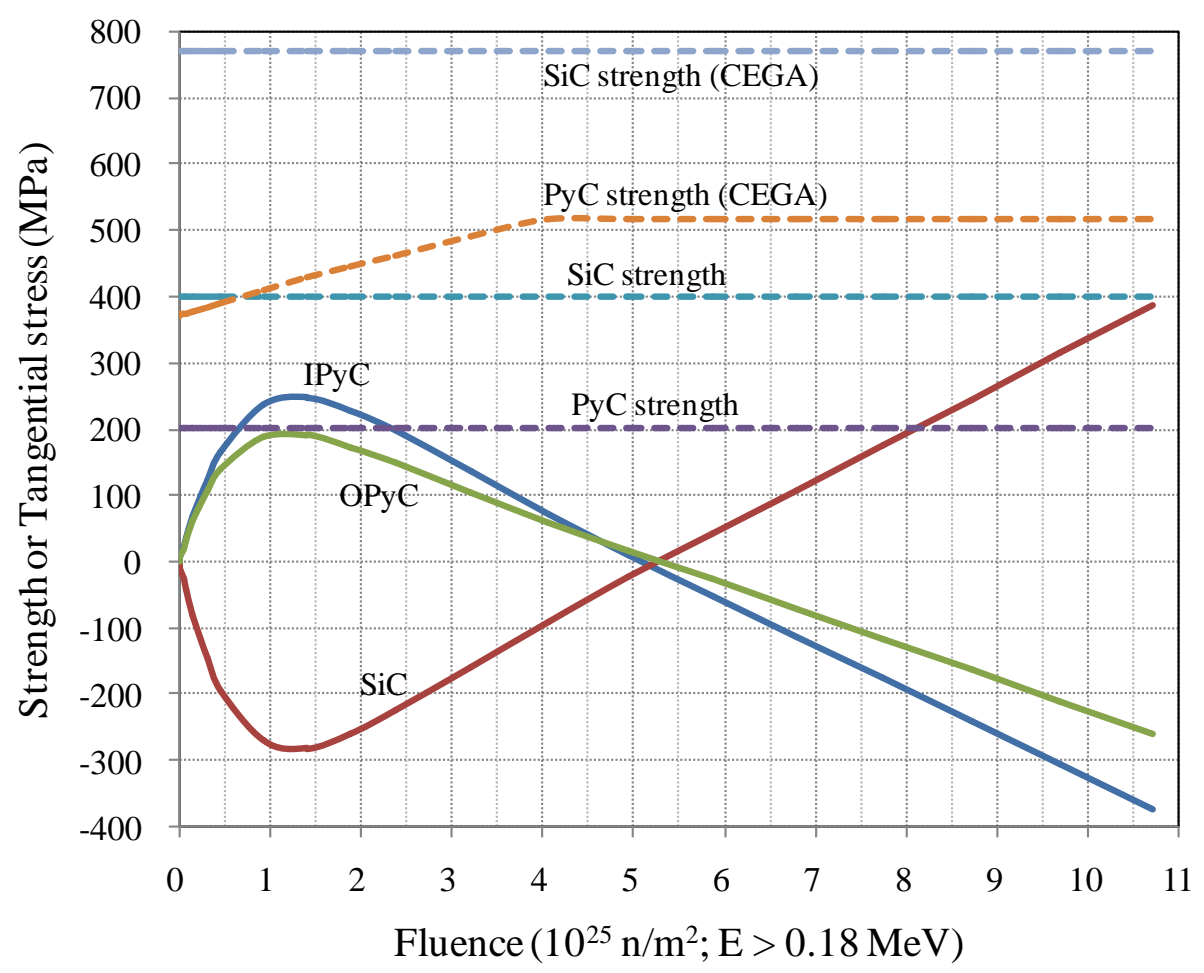

Fig. 69. Stress evolutions during normal operation at $850^{\circ} \mathrm{C}$.

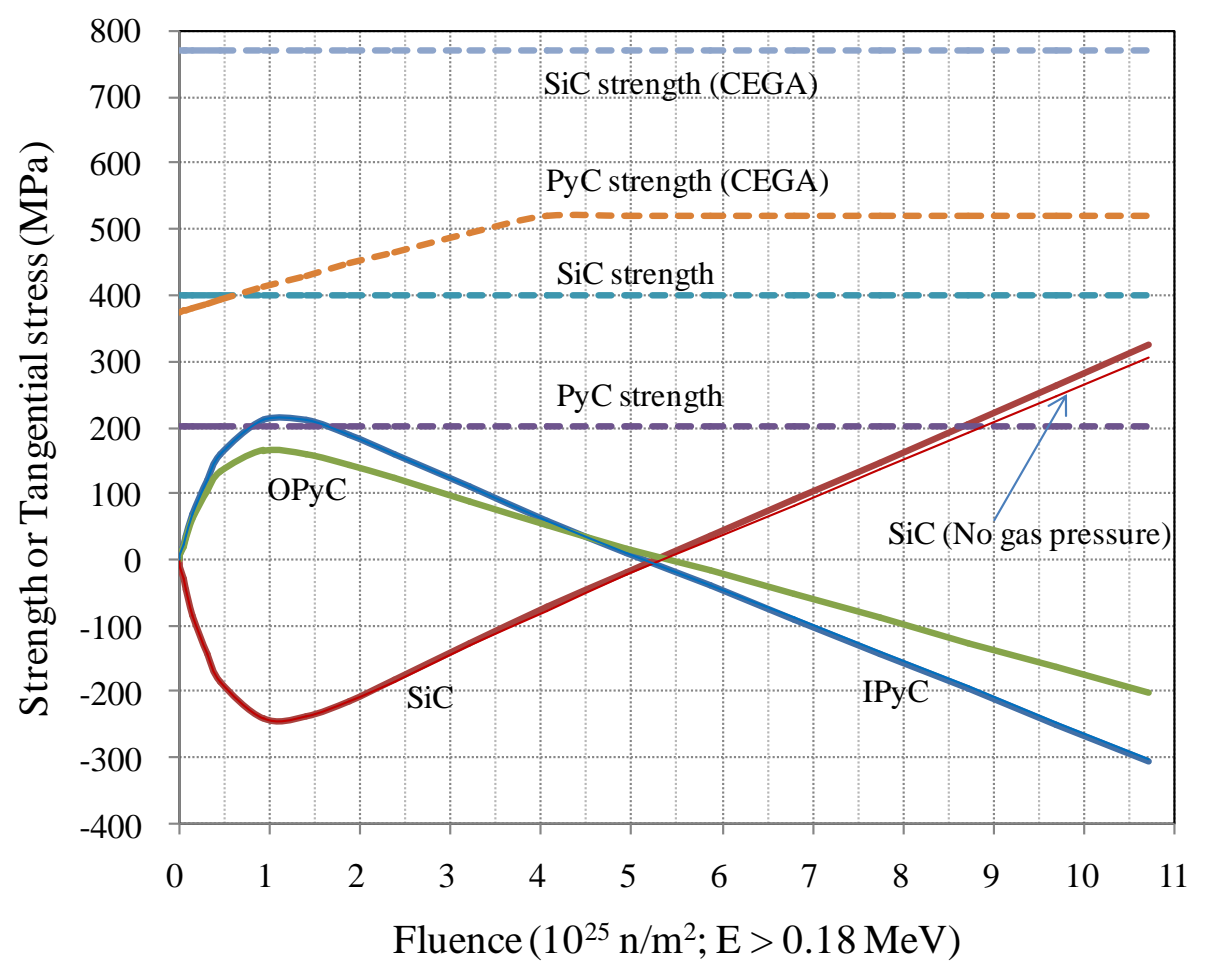

Fig. 70. Stress evolutions during normal operation at $950^{\circ} \mathrm{C}$. 


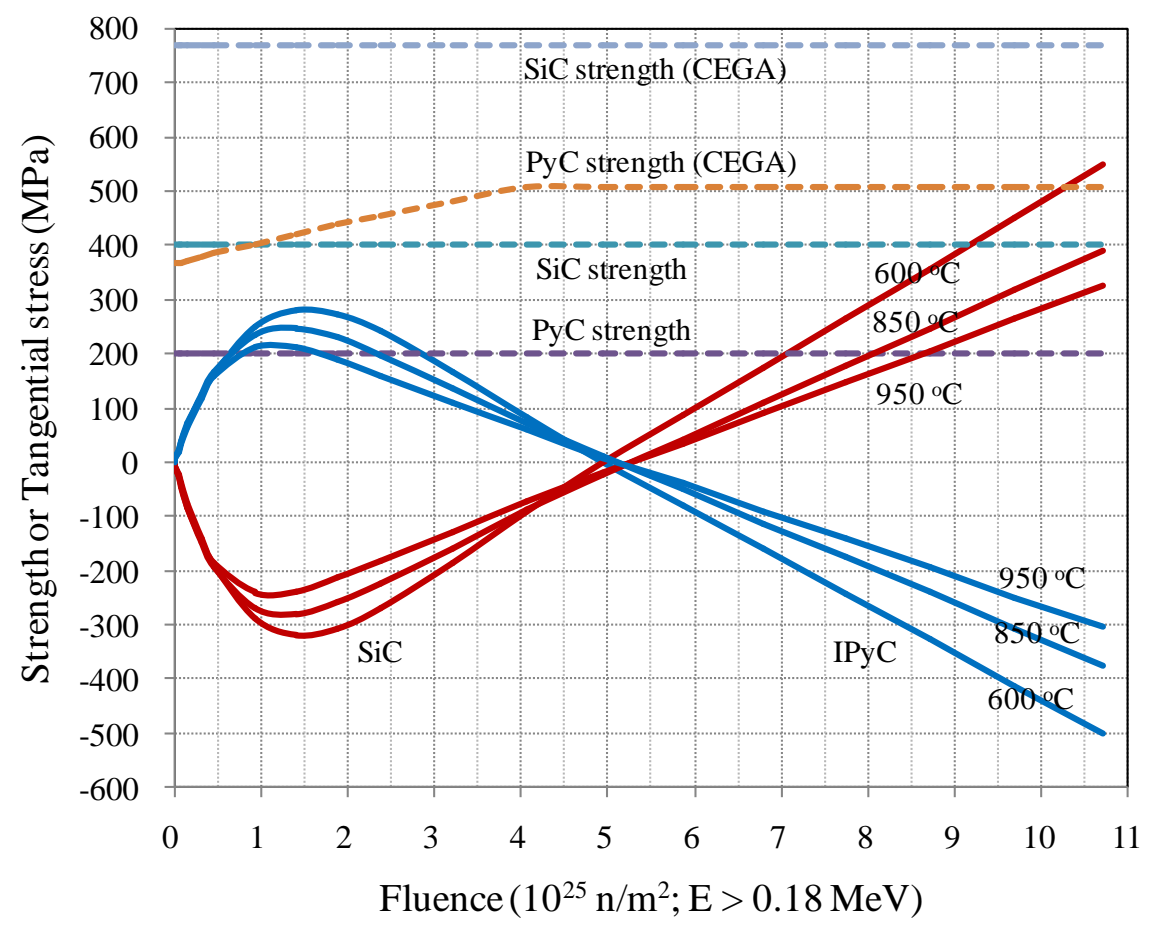

Fig. 71. Stress evolutions during normal operation.

\section{Failure Fraction of Coated Fuel Particles}

The failure of a coating layer occurs when the calculated stress acting on the layer is greater than the fracture strength of the layer. In this study, the fracture strengths for PyC and SiC are expressed as Weibull distributions, or just as mean fracture strength without statistical distribution. Figure 72 shows the failure fractions for $10^{5} \mathrm{CFPs}$ when the $\mathrm{PyC}$ and $\mathrm{SiC}$ strengths have Weibull distributions. There are no through-coating failures in these cases, meaning that one of the PyC layers survives during the SiC layer failure. On the other hand, the failure fractions for $10^{5} \mathrm{CFPs}$ are below $1.0 \times 10^{-5}$ when the PyC and $\mathrm{SiC}$ strengths are assumed to be 200 and $400 \mathrm{MPa}$, respectively. Figure 73 presents the stress behavior in a random CFP, in which the PyC layer broke at $1 \times 10^{21} \mathrm{n} / \mathrm{cm}^{2}(\mathrm{E}>0.18 \mathrm{MeV})$ while the stress of the $\mathrm{SiC}$ layer is much lower than its failure strength. 


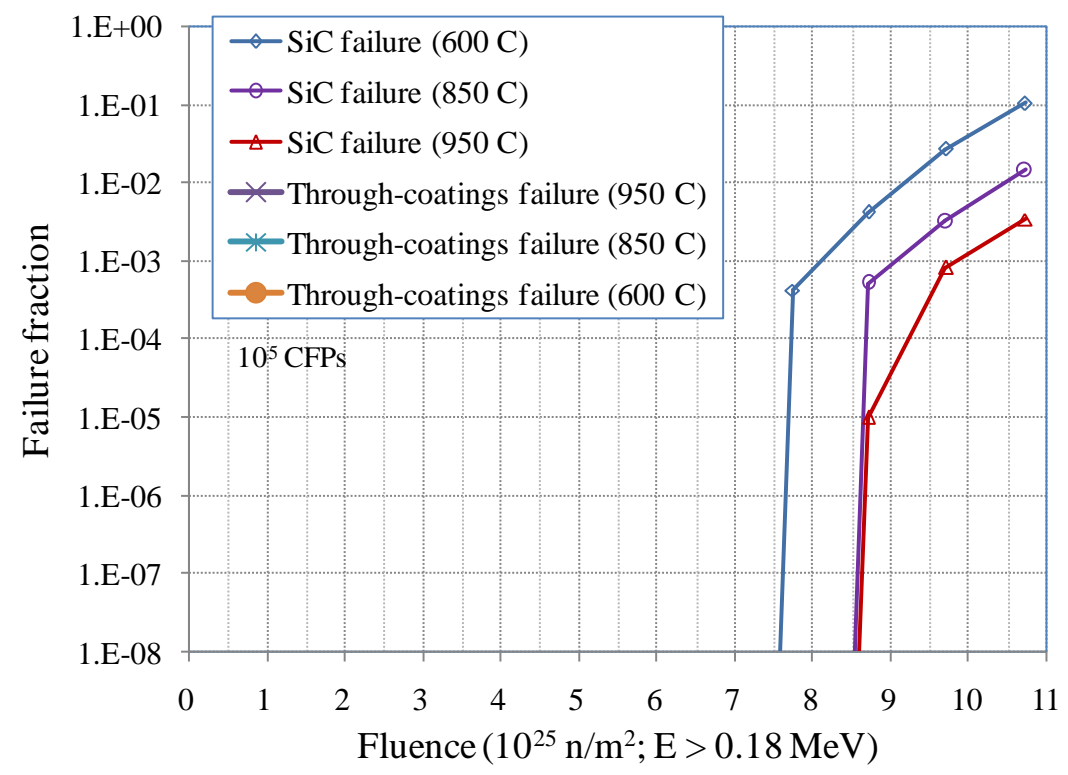

Fig. 72. Variation of failure fraction of CFPs.

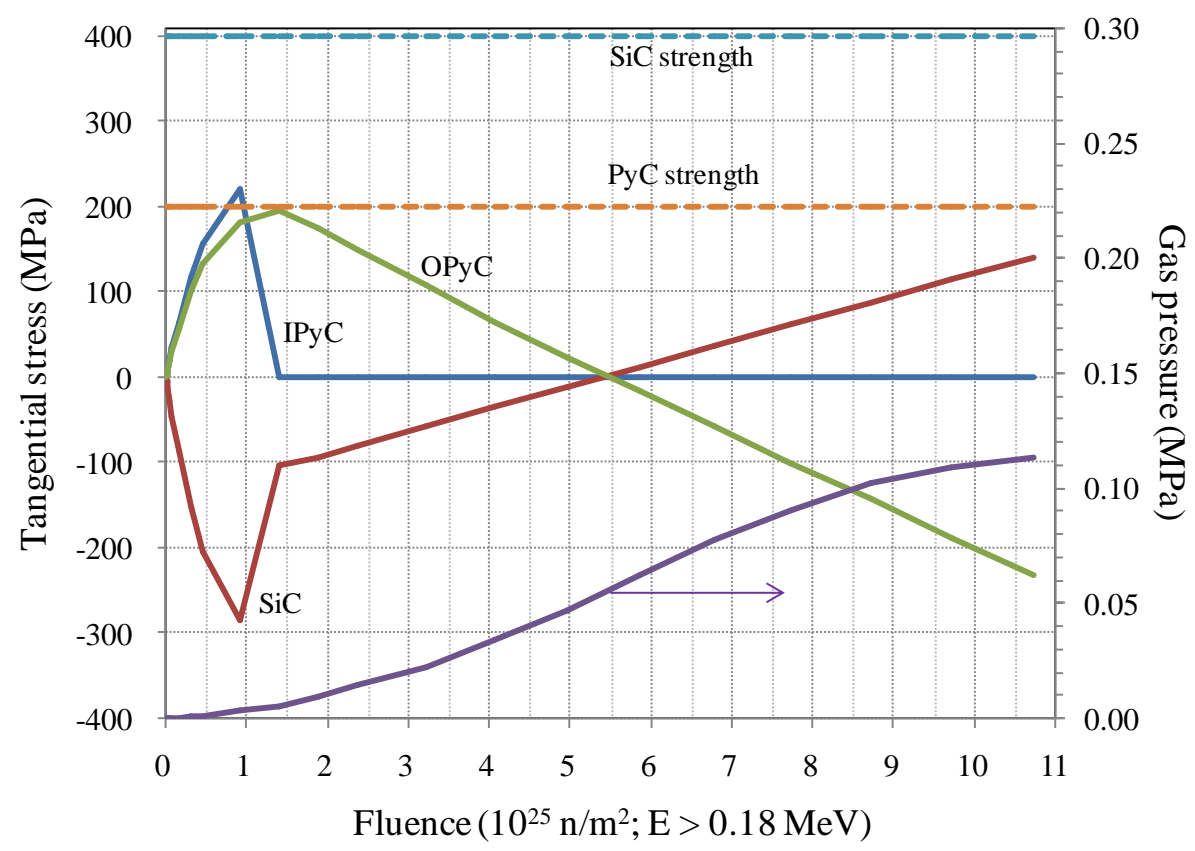

Fig. 73. Stress evolutions in a random CFP during normal operation. 


\section{Fuel Arraying and Rod Dimensioning, and Fuel Rod Thickness Determination}

The task aims to show the fuel feasibility and compatibility of the FCM fuel with existing Korean LWR cores (OPR-1000). This requires a new fuel design to compensate for the low fissile inventory of particlebased fuel and a comprehensive scoping analysis of neutronics, thermal hydraulics, and safety and fuel qualification aspects.

For a particle-based fuel, a thick-coated layer surrounding $\mathrm{UO}_{2}$ kernel retains released fission gas within the fuel particle element and significantly reduce fissile inventory per unit fuel volume. To accommodate the low fissile inventory, FCM fuel will be a "fat fuel" with tight inter-rod spacing. This section introduces and discusses some technical issues related to fuel arraying (fuel lattice formation) and fuel rod dimensioning (fuel rod diameter) of the FCM replacement fuel for LWRs, based on the Korean experience of developing dual-cooled annular fuel for existing LWRs.

\section{Fuel array and rod dimensioning}

To accommodate the existing control rods layout of the reference reactor (OPR-1000), the new FCM fuel lattice must have a square array of evenly located fuel rods of uniform diameter. Each fuel assembly has one center guide tube and four corner guide tubes. The candidate FCM fuel layouts are determined by the location and the shape of the guide tubes. Figure 74 shows three candidate fuel arrays of FCM replacement fuel, compared with the referenced $16 \times 16$ fuel array (at left). The candidate arrays were selected from the experience of developing dual-cooled annular fuel for existing LWRs (Chun, 2000).

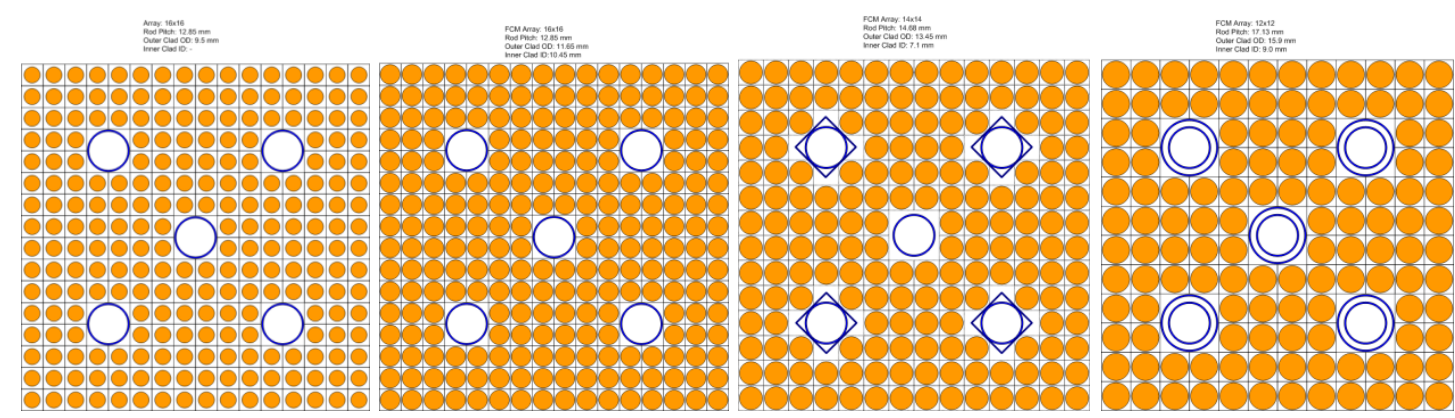

Fig. 74. FCM fuel array candidates $(16 \times 16$ conventional, $16 \times 16$ FCM, $14 \times 14$ FCM, $12 \times 12$ FCM fuel array, in order).

The adoption of a double circular guide tube relieves neutron moderation and water concentration due to the larger spacing around the guide tubes. If three fuel rods are located at the corner of guide tube in $14 \times 14$ fuel lattice, interference at the four sides of the guide tube occurs. Then, two fuel rods at four direct sides and three fuel rods at four diametrical corners of the guide tube were applied. The additional square guide of four corner guide tubes aimed for hydraulic balancing. A $16 \times 16$ fuel lattice has the same array as the existing LWR fuel assembly, but uses an enlarged rod diameter of rods. This configuration is superior in structural compatibility over the other candidates, but has thermal-hydraulic deficiencies. A larger array increases heat transfer area together with pressure loss. Furthermore, average heat flux decreases with increased heat transfer area.

In principle, fuel rod dimensioning requires iterative optimization from the comprehensive parametric studies of thermal-hydraulics and neutronics for the specific rod dimensions and arrays. Safety analysis will support and confirm the final optimized design decision. Here, in the fuel feasibility analysis stage, preliminary FCM fuel rod dimensions with the same fissile inventory to present fuel, according to the 
packing fraction and enrichment, can be proposed. Table 24 summarizes preliminary FCM rod diameters with equivalent fissile inventory to the current fuel design. The nominal percentage of flow area was compared with those of the FCM candidate dimension and arrays, referenced with that of the current fuel. A $12 \times 12$ FCM array with a $14.24 \mathrm{~mm}$ cladding diameter has a $12.6 \%$ difference in flow area, which is the lowest difference compared with the others.

Table 24. Preliminary FCM rod diameters with equivalent fissile inventory

\begin{tabular}{|c|c|c|c|c|c|c|c|}
\hline & $\begin{array}{c}16 \times 16 \\
\text { LWR }\end{array}$ & \multicolumn{7}{|c|}{$16 \times 16$ FCM, 12.85 mm pitch } \\
\hline Packing Frac.(\%) & & 50 & 40 & 30 & 50 & 40 & 30 \\
\hline Enrichment(\%) & 4.5 & \multicolumn{7}{|c|}{20} & \multicolumn{5}{|c|}{15} \\
\hline Pellet dia.(mm) & 8.19 & 9.38 & 10.49 & 12.11 & 10.83 & 12.11 & 13.98 \\
\hline clad dia.(mm) & 9.5 & 10.68 & 11.79 & 13.41 & 12.13 & 13.41 & 15.28 \\
\hline inter rod gap(mm) & 3.35 & 2.17 & 1.06 & - & - & - & - \\
\hline Flow area/FA (\%) & 100 & 82 & 76 & - & - & - & - \\
\hline
\end{tabular}

\begin{tabular}{|c|c|c|c|c|c|c|c|}
\hline & & \multicolumn{7}{|c|}{$14 \times 14$ FCM, 14.68 mm pitch } \\
\hline Pellet dia.(mm) & - & 10.83 & 12.11 & 13.98 & 12.51 & 13.98 & 16.15 \\
\hline clad dia.(mm) & - & 12.13 & 13.41 & 15.28 & 13.81 & 15.28 & 17.45 \\
\hline inter rod gap(mm) & - & 2.55 & 1.27 & - & - & - & - \\
\hline Flow area/FA (\%) & - & 84.50 & 77.60 & - & - & - & - \\
\hline
\end{tabular}

\begin{tabular}{|c|c|c|c|c|c|c|c|}
\hline & & \multicolumn{7}{|c|}{$12 \times 12$ FCM, 17.19 mm pitch } \\
\hline Pellet dia.(mm) & - & 12.94 & 14.47 & 16.71 & 14.94 & 16.71 & 19.29 \\
\hline Clad dia.(mm) & - & 14.24 & 15.77 & 18.01 & 16.24 & 18.01 & 20.59 \\
\hline Inter rod gap(mm) & - & 2.95 & 1.42 & - & - & - & - \\
\hline Flow area/FA (\%) & - & 87.40 & 78.73 & - & - & - & - \\
\hline
\end{tabular}

In summary, candidate fuel arrays and associated rod dimensions of FCM replacement fuel for LWRs were proposed based on the experience of dual-cooled annular fuel development. There was not much flexibility for choosing new fuel arrays that are compatible with existing power plants because of the strict restrictions of the control rods. Preliminary fuel rod diameters with the same fissile inventory to present fuel were proposed. A $12 \times 12 \mathrm{FCM}$ fuel array with $14.2 \mathrm{~mm}$ rod diameter has the minimum difference in flow area against that of present fuel.

\section{Fuel cladding thickness}

Determination of the FCM fuel rod thickness is based on the elastic buckling design criteria. Buckling load for the cladding is the pressure difference between the core coolant system and rod internal pressure. While the system pressure is constant during the reactor operation, the rod internal pressure changes with burn time period in the reactor because of fission gas release. The critical buckling pressure $\left(P_{c r}\right)$ for the elastic buckling is determined by Eq. (6-1).

$$
P_{c r}=\frac{E}{4(1-2 v)} \times\left(\frac{t}{r}\right)^{3}
$$

where $E$ is the elastic modulus, $v$ is Poisson's ratio, $t$ is the desired cladding thickness, and $r$ is the cladding radius. If the safety factor, $S$, is defined by the ratio of the critical pressure to the expected design load $\left(P_{D}\right)$ as Eq. $(6-2)$, 


$$
S=\frac{P_{c r}}{P_{D}}
$$

then the desired cladding thickness corresponding to the designated safety factor can be obtained. For instance, cladding $9.5 \mathrm{~mm}$ in diameter and $0.57 \mathrm{~mm}$ in thickness has $35.76 \mathrm{MPa}$ of the critical pressure, and then dividing by the system pressure gives a safety factor of 3.4 .

There are alternative two design options for use of cladding and the TRISO-coated particles: collapsible cladding and direct use of the TRISO-coated particles. Collapsible cladding is assessed to not be suitable for longer burnup LWR fuel with respect to structural integrity, dissolution problem (to LWR system coolant condition), and fretting wear. SS304 collapsible cladding $14.6 \mathrm{~mm}$ in diameter and $0.38 \mathrm{~mm}$ in thickness has a critical elastic buckling load of $5.3 \mathrm{MPa}$, which is too low, compared to $19.1 \mathrm{MPa}$ of the conventional fuel cladding $(15.15 \mathrm{~mm}$ in diameter and $0.59 \mathrm{~mm}$ in thickness with the same cladding material). While direct use of TRISO-coated particles without SiC matrix pelletizing can reduce fuel rod diameter by increasing packing fraction to $61 \%$, the low-density packing region of the upside of the fuel rod can be a potential source of collapse failure at the end of fuel lifetime and longer burnup.

\subsection{PLANNED ACTIVITIES FOR NEXT YEAR}

\subsubsection{Fuel Qualification}

Activities will continue as planned, including (1) continued FCM process development and properties investigation, (2) scaling the UN kernel fabrication to batch quantities leading to TRISO coating and compaction, and (3) irradiation of fueled FCM.

\subsubsection{Fuel Performance Modeling}

The planned fuel performance modeling are (1) the review of material property models justified under high fluence, (2) the development of performance analysis models for the failure mechanisms of a CFP under FCM LWR conditions, (3) the addition of a module analyzing fission product transport in an FCM LWR fuel rod to COPA.

\subsubsection{Scoping Analysis for OPR-1000}

The following analyses will be performed next year: the rigorous estimation of the failure fraction of more than $10^{8}$ CFPs including all possible failure mechanisms, the fractional release of fission products from CFPs into coolant, the thermo-mechanical analysis on pellet and cladding, and the flow-induced vibration analysis for dual batch in the transition core. These will contribute to the determination of the dimensions of an FCM LWR fuel rod, such as CFP size, gap width, cladding thickness.

\subsubsection{Scoping Analysis for WH Plants}

The following analyses will be performed next year: the estimation of the failure fraction of CFPs and the fractional release of fission products from CFPs into coolant.

\subsection{SUMMARY}

In the first year of this I-NERI, and in parallel with ongoing activities to develop the FCM fuel, significant progress has been made in (1) overcoating of fuel to address the specific fabrication issues, (2) optimization of the FCM matrix for density and thermal conductivity, (3) successful demonstration of a 
route to highly dense UN kernel fabrication, and (4) positive results from the first series of irradiation capsules targeting the stability of the FCM matrix material and the investigation of fuel/clad interaction.

The results of the FCM fuel performance analysis in LWR operating conditions can be summarized as follows.

1. Gas pressures at 600,850 , and $950^{\circ} \mathrm{C}$ are below $4 \mathrm{MPa}$ up to $11 \times 10^{21} \mathrm{n} / \mathrm{cm}^{2}(\mathrm{E}>0.18 \mathrm{MeV})$. The major gas species are xenon and krypton below $910^{\circ} \mathrm{C}$, but cesium becomes the major gas species above $910^{\circ} \mathrm{C}$.

2. Material properties are known below $4 \times 10^{21} \mathrm{n} / \mathrm{cm}^{2}(\mathrm{E}>0.18 \mathrm{MeV})$. A parabolic correlation was adopted describing an IIDC of high-density PyC over $4 \times 10^{21} \mathrm{n} / \mathrm{cm}^{2}(\mathrm{E}>0.18 \mathrm{MeV})$. The IPyC is likely to break at the early stage of irradiation when the PyC strength is $200 \mathrm{MPa}$, and the SiC layer may break just after $9 \times 10^{21} \mathrm{n} / \mathrm{cm}^{2}(\mathrm{E}>0.18 \mathrm{MeV})$ when the $\mathrm{SiC}$ strength is $400 \mathrm{MPa}$. The early failure of the IPyC layer can increase the integrity of the SiC layer.

3. The $\mathrm{SiC}$ failure fraction for $10^{5} \mathrm{CFPs}$ drastically increases near $8 \times 10^{21} \mathrm{n} / \mathrm{cm}^{2}(\mathrm{E}>0.18 \mathrm{MeV})$ when the PyC and SiC strengths are assumed to have Weibull distributions. However, one of the PyC layers will likely remain intact during the $\mathrm{SiC}$ failure. No failure occurs for $10^{5} \mathrm{CFPs}$ when the PyC and $\mathrm{SiC}$ failure strengths are given as 200 and $400 \mathrm{MPa}$, respectively.

4. The $12 \times 12$ FCM fuel array with $14.2 \mathrm{~mm}$ rod diameter has the minimum difference in flow area compared with the reference fuel and is compatible with fuel assembly manufacturing.

\subsection{REFERENCES}

ABAQUS, Inc. 1998. ABAQUS, Ver. 6.5-3. ABAQUS, Inc., U.S.A.

Arita, I.H., D.S. Wilkinson, and G.R. Purdy. 1992. J. Am. Ceram. Soc., 75, 3315-3320 (1992).

Beck, S.D. 1960. The Diffusion of Radioactive Fission Products from Porous Fuel Elements, BMI-1433.

CEGA Corporation. 1993. NP-MHTGR Material Models of Pyrocarbon and Pyrolytic Silicon Carbide, CEGA-002820, Rev. 1.

Chun, T.H. et al. 2000. Feasibility Study on Dual-Cooled Annular Fuel for OPR-1000 Power Uprate, KAERI/RR-3152/2009.

DeMange, P., J. Marian, M. Caro, and A. Caro. 2010. "TRISO-fuel Element Thermo-mechanical Performance Modeling for the Hybrid LIFE Engine with Pu Fuel Blanket," Journal of Nuclear Materials, 405, 144-155.

Dong, S., Y. Katoh, and A. Kohyama. 2003. J. Am. Ceram. Soc. 86(1), 26-32 (2003).

Hayes, S.L., J.K. Thomas, and K.L. Peddicord. 1990a. "Material Property Correlations for Uranium Mononitride II. Mechanical properties,” Journal of Nuclear Materials, 171, 271-288.

Hayes, S.L., J.K. Thomas, and K.L. Peddicord. 1990b. "Material Property Correlations for Uranium Mononitride I. Physical properties,” Journal of Nuclear Materials, 171, 262-270.

Hayes, S.L., Thomas, J.K., Peddicord, K.L., 1990c. "Material Property Correlations for Uranium Mononitride III. Transport properties," Journal of Nuclear Materials, 171, 289-299.

Hunn, J.D. and R.A. Lowden. 2006. Data compilation for AGR-1 baseline coated particle composite LEU01-46T, ORNL/TM-2006/019. 
Hunn, J.D., C.M. Barnes, J.A. Phillips. 2010. “Comparison of AGR-2 and AGR-1 UCO fuel,” HTR 2010 5th International Topical Meeting on High Temperature Reactor Technology, Prague, October 18-20, 2010.

Hunter, R.J. 1988. Zeta potential in colloid science, Academic Press, 1988.

IAEA. 1997. Fuel Performance and Fission Product Behavior in Gas Cooled Reactors, IAEA-TECDOC978.

INEEL, CEA, MIT. 2004. Development of Improved Models and Designs for Coated-Particle Gas Reactor Fuels, INEEL/EXT-05-02615, Idaho National Engineering and Environmental Laboratory.

IAEA. 2012. Advances in High Temperature Gas Cooled Reactor Fuel Technology, IAEA-TECDOC1674.

Katoh, Y.S., M. Dong, and A. Kohyama. 2002. "Thermo-mechanical properties and microstructure of silicon carbide composites fabricated by nano-infiltrated transient eutectoid process," Fus. Eng. Design, 61, 723 (2002).

Kim, Y.M., M.S. Cho, Y.W. Lee, and W.J. Lee. 2008. "Development of a Fuel Performance Analysis Code COPA," Paper 58040 in Proceedings of 4th International Conference on High Temperature Reactor Technology HTR 2008, Washington, DC, USA, 28 September - 1 October.

Kim, Y.M. and M.S. Cho. 2009. "A Stress Analysis for a Coated Fuel Particle of a HTGR using a Finite Element Method," Nuclear Engineering and Technology, 41(8), 1087-1100.

Kim, Y.M. 2012. A Finite Element Analysis of Fission Product Transport in a Fuel Block of a High Temperature Reactor, KAERI/TR-4551/2012, Korea Atomic Energy Research Institute.

Koyanagi, T., S. Kondo, and T. Hinoki. 2011. J. Nucl. Mater., 417, 435-439 (2011).

Maalmi M. and A. Varma. 1996. AIChE Journal, 42(12), 3477 (1996).

Mah, T. and M.D. Petry. 1992. J. Am. Ceram. Soc., 75, 2006-2009 (1992).

Miller, G.K., D.A. Petti, J.T. Maki, and D.L. Knudson. 2009. PARFUME Theory and Model Basis Report, INL/EXT-08-14497.

Nickel, H., H. Nabielek, G. Pott, and A.W. Mehner. 2002. Nucl. Eng. Des. 217, 141-151 (2002).

Omori, M. and H. Takei. 1982. J. Am. Ceram. Soc., 65, 92 (1982).

Outotec. 2011. HSC Chemistry Version 7.14. Outotec, Research Center, Finland.

Ozawa, K., Y. Katoh, L.L. Snead, T. Nozawa, T. Hinoki, and A. Hasegawa. 2009. "The effects of neutron irradiation on dimensional change in advanced silicon carbide composites," Fusion Materials Semiannual Progress Report for the Period Ending June 2009, DOE/ER-0313/46, pp. 4-15.

Park, J.S., et al. 2007. J. Nucl. Mater., 367-370, 719-724 (2007). 
Pepper, D.W. and J.C. Heinrich. 1992. The Finite Element Method, Hemisphere Publishing Corporation, USA.

Phillips, J., C. Barnes, and J. Hunn. 2010. "Fabrication and comparison of fuels for advanced gas reactor irradiation tests," in Proceedings of HTR 2010, Prague, Czech Republic, October 2010, paper 236.

Ross, S.B. and M.S. El-Genk. 1990. "Uranium Nitride Fuel Swelling Correlation," Journal of Nuclear Materials, 170, 169-177.

Sha, J.J., T. Nozawa, J.S. Park, Y. Katoh, and Y. Kohyama. 2004. J. Nucl. Mater., 329-333, 592 (2004).

Shimoda, K., J.S. Park, T. Hinoki, and A. Kohyama. 2007. "Densification mechanism and microstructural evolution of $\mathrm{SiC}$ matrix in NITE process: ceramics in nuclear and alternative energy applications,"

Ceram. Eng. Sci. Proc., 27(5) (2007).

Shimoda, K. 2008. Development of $\mathrm{SiC} / \mathrm{SiC}$ composite using ultrafine $\mathrm{SiC}$ powder, $\mathrm{PhD}$ Thesis, Kyoto University, 2008.

Shimoda, K., et al. 2009a. J. Nucl. Mater., 386-388, 634-638 (2009).

Shimoda, K., et al., 2009b. Compos. Sci. Technol., 69, 1623-1628 (2009).

Sigl, L.S. and H. Kleebe. 1993. J. Am. Ceram. Soc., 76(3), 773-776 (1993).

Snead, L.L. and T.D. Burchell. 1995. J. Nuc. Mater., 224, 222 (1995).

Snead, L.L., et al. 2007. "Handbook of SiC properties for fuel performance modeling," Journal of Nuclear Materials, 371, 329 (2007).

Snead, L.L., T.D. Burchell, and Y. Katoh. 2008. J. Nuc. Mater., 381, 55 (2008).

Snead, L.L., K.A. Terrani, F. Venneri, Y. Kim, J.E. Tulenko, C.W. Forsberg, P.F. Peterson, and E.J. Lahoda. 2011. "Fully Ceramic Microencapsulated Fuels: A Transformational technology for Present and Next Generation Reactors-Properties and Fabrication of FCM Fuel," Transactions of the American Nuclear Society, 104, 668 (2011).

Sun, J. and L. Gao. 2001. J. Eur. Ceram. Soc., 21, 2447-2451 (2001).

Terrani, K.A., J.O. Kiggans, Y. Katoh, K. Shimoda, F.C. Montgomery, B.L. Armstrong, C.M. Parish, T. Honoki, J.D. Hunn, and L.L. Snead. 2012a. "Fabrication and Characterization of Fully Ceramic Microencapsulated Fuels," Journal of Nuclear Materials, 426, 268-276 (2012).

Terrani, K.A., L.L. Snead, and J.C. Gehin. 2012b. "Microencapsulated fuel technology for commercial light water and advanced reactor applications," J. Nucl. Mater., 427, 209-224 (2012).

Zhou, Y., K. Hirao, M. Toriyama, Y. Yamauchi, and S. Kanzaki. 2001. J. Am. Ceram. Soc., 84(7), 16421644 (2001). 


\section{ISSUES AND CONCERNS}

There are several technical issues to be addressed.

1. Fast neutron fluence of the FCM fuel at the fuel assembly level appears to be slightly higher than that of the conventional fuel. It is expected that the full core analysis will reduce the fluence level by taking into account the realistic age and power level.

2. High pressure drop of the FCM fuel with larger rod diameter may deteriorate the DNBR and cross flow performance in the transition cores. An advance spacer grid design with a high DNBR performance and/or low pressure drop is required.

3. Safety criteria of the new concept FCM fuel design are not well established. Safety experiments are required to be performed to quantify these criteria in the longer term.

4. The behavior of the FCM fuel materials is not well known under LWR operating conditions. It is necessary to secure new material properties and irradiation performance data produced under extended fluence and over the expected temperature range.

The primary concerns related to fuel qualification are threefold. Thus far, the irradiation studies indicate that the matrix is quite stable under the conditions studied and that there is no fuel-clad interaction.

However, near-term concerns to be considered are as follows.

1. The FCM process lends itself to intermediate to large part scale fabrication. Small cylinder fabrication is challenging.

2. The UN kernel development, while initially encouraging, is still a development program and needs to be demonstrated at the batch scale and beyond, as well as the irradiation stability of UN at LWRrelevant temperatures.

3. Funding for such an aggressive concern is an issue. 


\section{CONCLUSIONS}

FCM fuel assembly designs that can replace conventional $\mathrm{UO}_{2}$ fuel assemblies are proposed and evaluated for their compatibility with existing $16 \times 16$ and $17 \times 17$ fuel assembly cores. Candidate fuel assembly designs are first screened based on the design screening criteria. Screening criteria are set up to have similar core reactivity, cycle length, and reactivity coefficients, and to have compatible pressure drop and DNBR characteristics, while maintaining mechanical compatibility with existing core and fuel manufacturing capability. Feasible designs are then selected by quantitative analysis based on their neutronic, thermal-hydraulic, and mechanical compatibility with the existing cores. Then the fuel performance over lifetime and the safety of the FCM fueled cores are evaluated.

Analysis methods and models applicable to the FCM fuel are established and applied for the preliminary analysis of neutronics, thermal-hydraulics, fuel performance and safety compatibilities. In parallel, UCO FCM fuel samples are manufactured and irradiated up to $8.5 \mathrm{dpa}$ for future quantification of enhanced material properties and integrity.

The following summarizes the results of the first year study.

\section{$\underline{\text { Task 1: Neutronic Exploration }}$}

- $\quad$ Analysis method: DeCART2D/MASTER

- Four feasible fuel designs are selected through scoping analysis at fuel assembly level - UN FCM fuel in $12 \times 12$ and $16 \times 16$ arrays with SS and SiC as cladding materials. These designs will be confirmed by the full-core performance analysis in the next fiscal year.

\section{Task 2: Core Thermal-Hydraulic Assessment}

- Analysis method: MATRA

- Preliminary scoping analyses on the pressure drop and DNBR performance of the FCM fuel assembly are carried out for various combinations of the assembly pitch-to-diameter ratio and the spacer grid designs. The results are used to select feasible fuel assembly designs that are thermalhydraulically compatible with the existing core. Detailed subchannel analysis for homogeneous and transition cores will follow in the next fiscal year.

\section{Task 3: Safety Assessment}

- Analysis method: MARS/MASTER

- Safety of the FCM fueled core is assessed to have sufficient margin to preliminary safety criteria through DBA analysis for LOFA, LOCA, and REA. Analysis results of the extreme BDBAs such as LOCA without SI and SBO show that the FCM fuel can survive longer and thus can provide a longer time for operators' mitigation actions than the conventional fuel. Safety analysis for the transition core with coupled and sophisticated radiation heat transfer will be performed in the next fiscal year.

\section{Task 4: Fuel Qualification}

- Fuel qualification: In parallel with ongoing activities to develop the FCM fuel, significant progress has been made in (1) overcoating of fuel to address the specific fabrication issues, (2) optimization of the FCM matrix for density and thermal conductivity, (3) successful 
demonstration of a route to highly dense UN kernel fabrication, and (4) positive results from the first series of irradiation capsules targeting the stability of the FCM matrix material and the investigation of fuel/clad interaction.

- Fuel performance assessment: The COPA/ABAQUS analysis system has been established and applied to the scoping analysis of FCM fuel performance. The FCM TRISO performance over the lifetime for the selected FCM TRISO has been assessed using preliminary material properties. Fission gas pressures (including noble gases and vapor species) at various operating conditions are maintained well below $4 \mathrm{MPa}$ mainly contributed to by xenon and krypton. However, statistical $\mathrm{SiC}$ failure rate increases near $8 \mathrm{dpa}$ of fluence. The $\mathrm{SiC}$ failure rate is very sensitive to the irradiation properties of the internal PyC that are not well quantified at higher fluences over 4 dpa. Refinement of analysis models will follow in the next fiscal year based on the irradiation data of the FCM fuel. 